\title{
v-ATPase is a key player in lipid-induced cardiomyopathy
}

Citation for published version (APA):

Liu, Y. (2016). v-ATPase is a key player in lipid-induced cardiomyopathy. [Doctoral Thesis, Maastricht University]. Maastricht University. https://doi.org/10.26481/dis.20160913yl

Document status and date:

Published: 01/01/2016

DOI:

10.26481/dis.20160913yl

Document Version:

Publisher's PDF, also known as Version of record

\section{Please check the document version of this publication:}

- A submitted manuscript is the version of the article upon submission and before peer-review. There can be important differences between the submitted version and the official published version of record.

People interested in the research are advised to contact the author for the final version of the publication, or visit the DOI to the publisher's website.

- The final author version and the galley proof are versions of the publication after peer review.

- The final published version features the final layout of the paper including the volume, issue and page numbers.

Link to publication

\footnotetext{
General rights rights.

- You may freely distribute the URL identifying the publication in the public portal. please follow below link for the End User Agreement:

www.umlib.nl/taverne-license

Take down policy

If you believe that this document breaches copyright please contact us at:

repository@maastrichtuniversity.nl

providing details and we will investigate your claim.
}

Copyright and moral rights for the publications made accessible in the public portal are retained by the authors and/or other copyright owners and it is a condition of accessing publications that users recognise and abide by the legal requirements associated with these

- Users may download and print one copy of any publication from the public portal for the purpose of private study or research.

- You may not further distribute the material or use it for any profit-making activity or commercial gain

If the publication is distributed under the terms of Article $25 \mathrm{fa}$ of the Dutch Copyright Act, indicated by the "Taverne" license above, 


\section{$\mathrm{v}$-ATPase is a key player in lipid-induced cardiomyopathy}


(C) Yilin Liu, 2016. For all articles published, the copyright has been transferred to the respective publisher. No part of this publication may be reproduced, stored in a retrieval system or transmitted in any form by any means, without prior written permission from the author, or when appropriate, from the publisher.

ISBN: 978-90-9029892-4

Cover design and layout by: Tomasz Karawajczyk

Financial support by the Dutch Heart Foundation for the publication of this thesis is gratefully acknowledged.

Publication of this thesis is further supported by Stichting Hartsvrienden RESCAR. 


\section{$\mathrm{v}$-ATPase is a key player in lipid-induced cardiomyopathy}

PROEFSCHRIFT

ter verkrijging van de graad van doctor aan de Universiteit Maastricht, op gezag van de Rector Magnificus, Prof. Dr. R.M. Letschert, volgens het besluit van het College van Decanen, in het openbaar te verdedigen op dinstag 13 September 2016 om 16.15 uur

door

Yilin Liu

Geboren op 6 September 1987 te Changchun 


\section{Promotor}

Prof. dr. J.F.C. Glatz

\section{Co-promotores}

Dr. J.J.F.P. Luiken

Dr. D. Neumann

\section{Beoordelingscommissie}

Prof. dr. C.G. Schalkwijk (voorzitter)

Prof. dr. S. Lin (Xiamen University, China)

Prof. dr. E.E. Blaak

Dr. R.C. Langen

Dr. L.C. Heather (University of Oxford, United Kingdom) 
I believe that if life gives you lemons

you should make lemonade...

and try to find somebody

whose life has given them wodka

and have a party 



\section{Contents}

Chapter 1 General Introduction 9

Chapter 2 Molecular mechanism of lipid-induced cardiac insulin resistance and contractile dysfunction

Chapter 3 Central role of dysassembly of vacuolar-type $\mathrm{H}^{+}$-ATPase in 43 lipid-induced cardiomyopathy

Chapter 4 Fluorescent labeling of fatty acid transporter CD36 in the 75 extracellular loop

Chapter 5 Central role of dysassembly of vacuolar-type $\mathrm{H}^{+}$-ATPase in 93 lipid-induced cardiomyopathy

Chapter 6 General discussion

Appendices

Summary

Samenvatting

总结

Valorization

Curriculum vitae

Acknowledgments 

Chapter 1

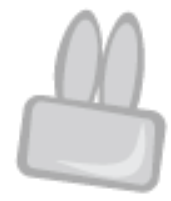

General Introduction 


\section{Introduction}

\section{Type 2 diabetes and diabetic cardiomyopathy}

Over the past 50 years, the prevalence of type 2 diabetes has dramatically increased to reach 285 million people in a global range, and this number is predicted to increase by about $50 \%$ by the year 2030 according to the International Diabetes Federation [1]. Highenergy food intake and reduced physical activity are regarded as the main causes of the increase in the prevalence of obesity and type 2 diabetes. Type 2 diabetes is characterized by high blood glucose levels caused by the body's inability to use insulin efficiently, a situation referred to as insulin resistance [2]. Compensatory hyperinsulinemia is often seen in pre-diabetic individuals and results from increasing insulin secretion from $\beta$-cells to meet the increased insulin demand. However, when $\beta$-cells are getting dysfunctional and, as a result, insulin secretion becomes insufficient to meet the high demand, type 2 diabetes develops [3]. Individuals with chronic overweight are commonly type 2 diabetic and have a high risk of developing diabetes-related complications. Cardiovascular disease is the major complication in type 2 diabetes, and is conjugated with many risk factors including hypertension, dyslipidemia, glucose intolerance as well as insulin resistance. This condition is referred to as diabetic cardiomyopathy [4].

\section{Role of CD36 in cardiac lipid accumulation and cardiac dysfunction}

A high-fat diet, especially a high intake of saturated fat, is a key contributor to type 2 diabetes. Typically, people adhering to a high-fat diet are obese and resistant to insulin. A major consequence of fat overconsumption is the accumulation of fatty acids and their metabolites in non-adipose tissues including liver, heart and skeletal muscle, which is increasingly being recognized as a major cause for insulin resistance. In the heart, this ectopic lipid accumulation ultimately leads to cardiac contractile dysfunction [5-7]. Such association between myocardial lipid accumulation and decreased contractile function is found in both humans $[5,8]$ and insulin resistant rat models $[5,6]$.

Cardiac lipid metabolism is mainly regulated by the membrane-associated protein CD36, which facilitates long-chain fatty acid uptake into the heart [7]. CD36, also known as fatty acid translocase (FAT), or 'fatty acid transporter', is a 472 -amino acid ( $88 \mathrm{KDa}$ ) membrane protein that has a hairpin structure with two transmembrane regions, with both C-terminal and N-terminal tails as short segments in the cytoplasm [7]. In the heart under resting conditions, it has been estimated that $\sim 50 \%$ of the CD36 population is stored in endosomes, from where it can translocate to the plasma membrane to increase fatty acid uptake. Insulin and other physiological stimuli induce CD36 translocation, and thereby stimulate myocardial long-chain fatty acid uptake and utilization [7]. This regulatory mechanism has now been well accepted and offers a further explanation for the 
A
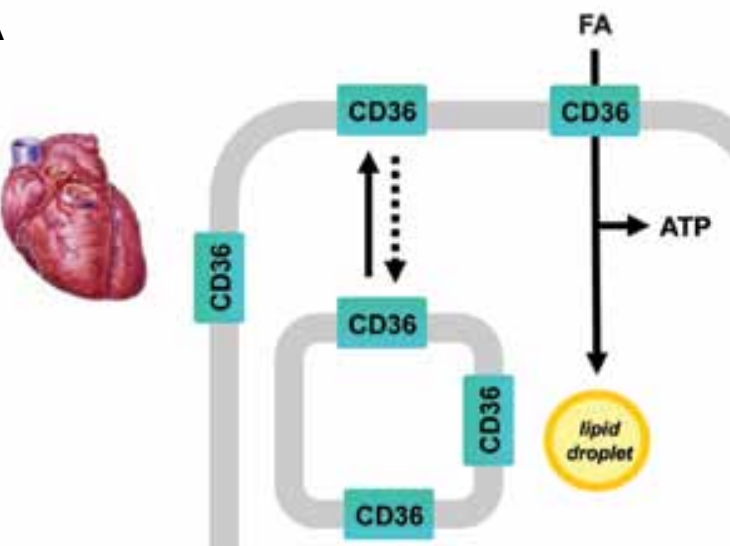

Healthy

B

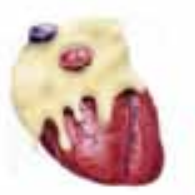

Dlabetic
FA

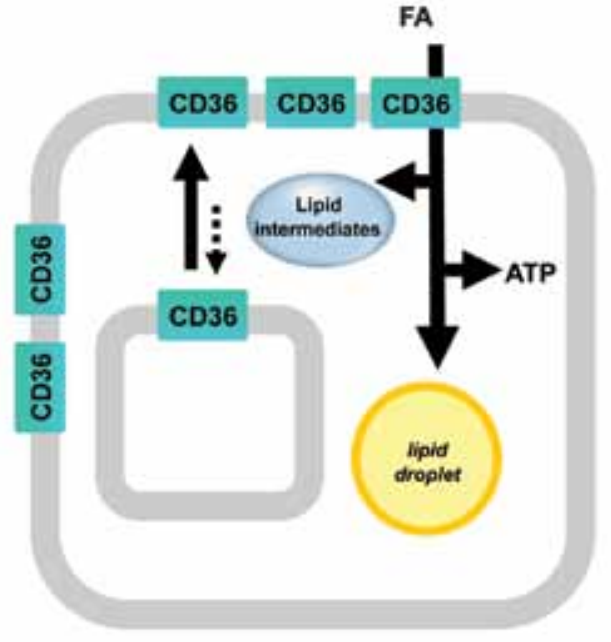

Figure 1. Schematic presentation of the regulation of fatty acid transporter CD36 in healthy versus diabetic heart. (A) In the healthy heart under non-stimulated condition, CD36 translocates from endosomes to the plasma membrane to increase fatty acid uptake. (B) In the diabetic status, CD36 abundance at the plasma membrane is markedly increased due to sustained relocation from endosomes. The aberrant high sarcolemmal CD36 presence causes a chronically elevated influx of fatty acid into cardiomyocytes, which is directly coupled to lipid accumulation in the heart.

emerging role of CD36 in metabolic diseases $[7,9,10]$.

Upon overexposure of the heart to lipids, CD36 permanently relocates to the sarcolemma, initiating a vicious cycle of lipid accumulation and lipid-induced insulin resistance, leading to cardiac dysfunction (Figure 1) $[7,11,12]$. The causal role of CD36 in the development of cardiac dysfunction in obesity, insulin resistance and type 2 diabetes has been further confirmed by CD36-null mice studies, which revealed that CD36 ablation 
is protective against loss of cardiac function in several models of lipid-induced cardiomyopathy [13-15]. Collectively, CD36 appears to be a main factor that is responsible for cardiac insulin resistance and contractile dysfunction in the lipid-overloaded heart.

\section{The endosomal proton pump: v-ATPase}

The preferential sarcolemmal localization of CD36 upon overexposure of the heart to lipids is most likely caused by alterations in CD36-dedicated trafficking. Like other cellular protein translocation processes, CD36 translocation is mediated by vesicles, and consists of three major steps: vesicle fission, subcellular vesicle transport, and vesicle fusion. A number of proteins are involved, such as coat proteins for budding in vesicle fission and fusion, Rab proteins for providing the unidirectionality in vesicle transport, and SNARE proteins for the specificity of trafficking $[7,16]$. Though CD36-containing vesicles have been isolated [17], the CD36-dedicated trafficking machinery has been largely uncharted.

Vacuolar $\mathrm{H}^{+}$-ATPase (v-ATPase) functions as ATP-driven proton pump and occurs in acidic organelles such as endosomes, i.e., the intracellular storage compartment for CD36 $[18,19]$. V-ATPase is entirely responsible for endosomal acidification, which is the main defining feature of the endosomal compartment. As a large multi-subunit protein, v-ATPase can be functionally divided into two distinct sub-complexes: membrane subcomplex $\mathrm{V}_{0}(260 \mathrm{KDa})$ and cytoplasmic sub-complex $\mathrm{V}_{1}(650 \mathrm{KDa})$, encompassing the proton channel and catalyzing ATP binding, respectively (Figure 2) [20]. A previous study demonstrated that upon exposure of cardiomyocytes to the potent v-ATPase inhibitor bafilomycin-A, CD36 was expelled from the endosomes to the sarcolemma whereas other endosomally stored proteins, i.e., GLUT4, were retained, thus indicating the involvement of v-ATPase in CD36-dedicated trafficking [21]. Because endosomal alkalinization was found to correlate to CD36 relocation to the cell surface [21], the $\mathrm{pH}$ changes in the endosomes might play a role in the lipid-induced increase of the sarcolemmal CD36 content. Taken together, we speculate that lipids could affect v-ATPase function thereby altering CD36 translocation to the sarcolemma.

\section{Outline of the thesis}

In this thesis, a novel link between v-ATPase and myocellular insulin resistance upon lipid oversupply is disclosed. Furthermore, the mechanism of v-ATPase involvement was investigated and new therapeutic concepts to restore insulin sensitivity and contractile function in the heart are devised from these findings. Chapter 2 gives an overview of current mechanistic insights into the downstream events of CD36 relocation in the lipidoverloaded heart leading to contractile dysfunction. Despite intensive research has been conducted, the mechanisms by which lipids induce cardiac contractile dysfunction remain presently only partly understood. To reveal the mechanism of lipid-induced cardiac 


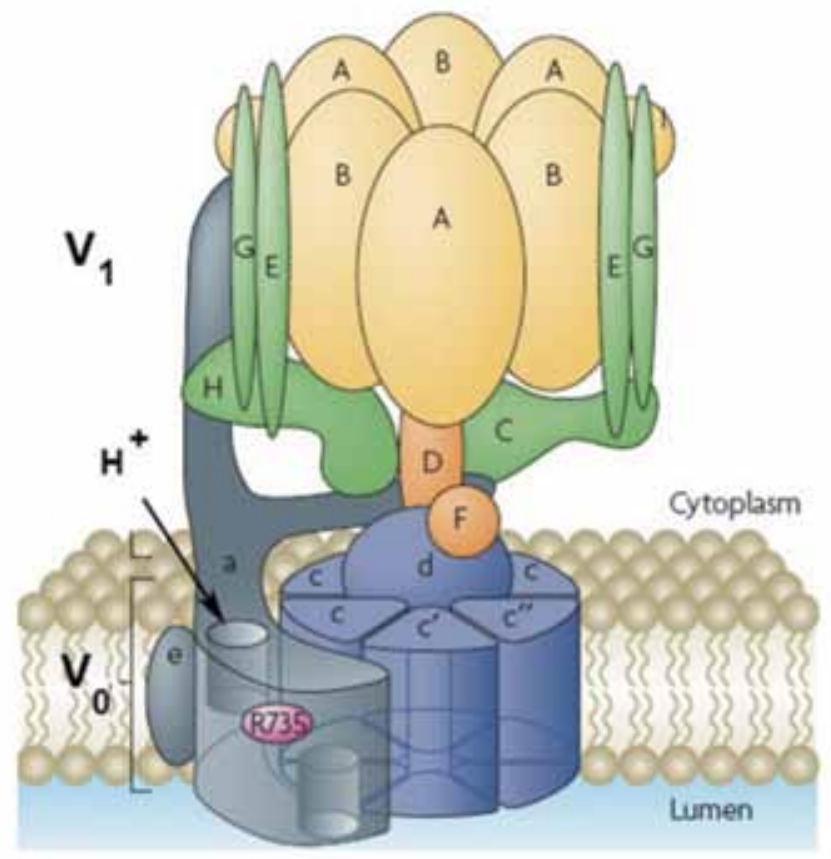

Figure 2. Structure of v-ATPase. Vacuolar H+-ATPase (v-ATPase) consists of 14 subunits: 6 subunits (a, e, c, c', c' and d) form the integral membrane-associated sub-complex V0 (shown in blue and grey), which is responsible for encompassing the proton channel. 8 subunits (A, B, C, D, E, F, G, and H) make up a peripheral membrane sub-complex V1 (shown in yellow, green and orange), which participates in ATP binding and hydrolysis. The V1 and V0 sub-complexes are connected by a central stalk (subunits D, F, and d) and multiple peripheral stalks (subunits C, E, $\mathrm{G}, \mathrm{H}$ and $\mathrm{a}$ ).

Taken with permission from Forgac M, Nat Rev Mol Cell Biol 8 (2007) 917-929.

dysfunction upstream of CD36 relocation, we examined the involvement of v-ATPase, which is described in Chapter 3. These findings provide evidence for a causal role of v-ATPase in lipid-induced cardiomyopathy. Chapter 4 establishes a novel approach to fluorescently label CD36 in the extracellular loop, which offers a possibility to study CD36 trafficking dynamics and allowed to confirm assembly/disassembly of v-ATPase as a potential mechanism in lipid-induced v-ATPase dysfunction and CD36 relocation. Since the reversible assembly/disassembly of $\mathrm{v}$-ATPase is regulated by glucose, as described in yeast, Chapter $\mathbf{5}$ addresses the restoration of v-ATPase activity as a possible measure to attain endosomal CD36 localization, and insulin sensitivity in the heart despite lipid oversupply. Finally, the findings of this thesis are discussed in Chapter 6, linking the outcome to the current state of this field and giving a broader perspective. 


\section{References}

1. S. Wild, G. Roglic, A. Green, R. Sicree, H. King, Global prevalence of diabetes: estimates for the year 2000 and projections for 2030, Diabetes Care, 27 (2004) 1047-1053.

2. V.A. Fonseca, Defining and characterizing the progression of type 2 diabetes, Diabetes Care, 32 Suppl 2 (2009) S151-156.

3. M. Cnop, J. Vidal, R.L. Hull, K.M. Utzschneider, D.B. Carr, T. Schraw, P.E. Scherer, E.J. Boyko, W.Y. Fujimoto, S.E. Kahn, Progressive loss of beta-cell function leads to worsening glucose tolerance in first-degree relatives of subjects with type 2 diabetes, Diabetes Care, 30 (2007) 677-682.

4. S. Rubler, J. Dlugash, Y.Z. Yuceoglu, T. Kumral, A.W. Branwood, A. Grishman, New type of cardiomyopathy associated with diabetic glomerulosclerosis, Am J Cardiol, 30 (1972) 595602.

5. R.H. Unger, L. Orci, Diseases of liporegulation: new perspective on obesity and related disorders, FASEB J, 15 (2001) 312-321.

6. S. Sharma, J.V. Adrogue, L. Golfman, I. Uray, J. Lemm, K. Youker, G.P. Noon, O.H. Frazier, H. Taegtmeyer, Intramyocardial lipid accumulation in the failing human heart resembles the lipotoxic rat heart, FASEB J, 18 (2004) 16921700.

7. J.F. Glatz, J.J. Luiken, A. Bonen, Membrane fatty acid transporters as regulators of lipid metabolism: implications for metabolic disease, Physiol Rev, 90 (2010) 367-417.

8. L.S. Szczepaniak, R.L. Dobbins, G.J. Metzger, G. Sartoni-D’Ambrosia, D. Arbique, W. Vongpatanasin, R. Unger, R.G. Victor, Myocardial triglycerides and systolic function in humans: in vivo evaluation by localized proton spectroscopy and cardiac imaging, Magn Reson Med, 49 (2003) 417-423.

9. [9] R. Palanivel, M. Eguchi, I. Shuralyova, I. Coe, G. Sweeney, Distinct effects of short- and long-term leptin treatment on glucose and fatty acid uptake and metabolism in HL-1 cardiomyocytes, Metabolism, 55 (2006) 10671075.

10. D. Samovski, X. Su, Y. Xu, N.A. Abumrad, P.D. Stahl, Insulin and AMPK regulate FA translocase/CD36 plasma membrane recruitment in cardiomyocytes via Rab GAP AS160 and Rab8a Rab GTPase, J Lipid Res, 53 (2012) 709717.

11. S.L. Coort, D.M. Hasselbaink, D.P. Koonen, J. Willems, W.A. Coumans, A. Chabowski, G.J. van der Vusse, A. Bonen, J.F. Glatz, J.J. Luiken,
Enhanced sarcolemmal FAT/CD36 content and triacylglycerol storage in cardiac myocytes from obese zucker rats, Diabetes, 53 (2004) 1655-1663.

12. D.M. Ouwens, M. Diamant, M. Fodor, D.D. Habets, M.M. Pelsers, M. El Hasnaoui, Z.C. Dang, C.E. van den Brom, R. Vlasblom, A. Rietdijk, C. Boer, S.L. Coort, J.F. Glatz, J.J. Luiken, Cardiac contractile dysfunction in insulin-resistant rats fed a high-fat diet is associated with elevated CD36-mediated fatty acid uptake and esterification, Diabetologia, 50 (2007) 19381948.

13. J. Yang, N. Sambandam, X. Han, R.W. Gross, M. Courtois, A. Kovacs, M. Febbraio, B.N. Finck, D.P. Kelly, CD36 deficiency rescues lipotoxic cardiomyopathy, Circ Res, 100 (2007) 12081217.

14. D.P. Koonen, M.M. Sung, C.K. Kao, V.W. Dolinsky, T.R. Koves, O. Ilkayeva, R.L. Jacobs, D.E. Vance, P.E. Light, D.M. Muoio, M. Febbraio, J.R. Dyck, Alterations in skeletal muscle fatty acid handling predisposes middle-aged mice to diet-induced insulin resistance, Diabetes, 59 (2010) 1366-1375.

15. L.K. Steinbusch, J.J. Luiken, R. Vlasblom, A. Chabowski, N.T. Hoebers, W.A. Coumans, I.O. Vroegrijk, P.J. Voshol, D.M. Ouwens, J.F. Glatz, M. Diamant, Absence of fatty acid transporter CD36 protects against Western-type diet-related cardiac dysfunction following pressure overload in mice, Am J Physiol Endocrinol Metab, 301 (2011) E618-627.

16. L.K. Steinbusch, R.W. Schwenk, D.M. Ouwens, M. Diamant, J.F. Glatz, J.J. Luiken, Subcellular trafficking of the substrate transporters GLUT4 and CD36 in cardiomyocytes, Cell Mol Life Sci, 68 (2011) 2525-2538.

17. H. Muller, K. Deckers, J. Eckel, The fatty acid translocase (FAT)/CD36 and the glucose transporter GLUT4 are localized in different cellular compartments in rat cardiac muscle, Biochem Biophys Res Commun, 293 (2002) 665-669.

18. B. Ma, Y. Xiang, L. An, Structural bases of physiological functions and roles of the vacuolar H(+)-ATPase, Cell Signal, 23 (2011) 12441256.

19. P.M. Kane, Targeting reversible disassembly as a mechanism of controlling V-ATPase activity, Curr Protein Pept Sci, 13 (2012) 117-123.

20. M. Forgac, Vacuolar ATPases: rotary proton pumps in physiology and pathophysiology, Nat Rev Mol Cell Biol, 8 (2007) 917-929. 
21. L.K. Steinbusch, W. Wijnen, R.W. Schwenk, W.A. Coumans, N.T. Hoebers, D.M. Ouwens, W.A. Coumans, N.T. Hoebers, M. Diamant, A. Bonen, J.F. Glatz, J.J. Luiken, Differential regulation of cardiac glucose and fatty acid uptake by endosomal $\mathrm{pH}$ and actin filaments, $\mathrm{Am} \mathrm{J}$ Physiol Cell Physiol, 298 (2010) C1549-1559. 



\section{Chapter 2}

\section{Molecular mechanism of lipid-induced cardiac insulin resistance and contractile dysfunction}

Yilin Liu, Dietbert Neumann, Jan F.C. Glatz ${ }^{\star}$, Joost J.F.P. Luiken

Department of Molecular Genetics, Cardiovascular Research Institute Maastricht (CARIM), Maastricht University, P.O. Box 616, 6200 MD Maastricht, The Netherlands

${ }^{\star}$ Corresponding author. Tel.: +31 433881998; fax: +31 433884574 . e-mail address: glatz@maastrichtuniversity.nl 


\begin{abstract}
Long-chain fatty acids are the main cardiac substrates from which ATP is generated continually to serve the high energy demand and sustain the normal function of the heart. Under healthy conditions, fatty acid $\beta$-oxidation produces $50-70 \%$ of the energy demands with the remainder largely accounted for by glucose. Chronically increased dietary lipid supply often leads to excess lipid accumulation in the heart, which is linked to a variety of maladaptive phenomena, such as insulin resistance, cardiac hypertrophy and contractile dysfunction. CD36, the predominant cardiac fatty acid transporter, has a key role in setting the heart on a road to contractile dysfunction upon the onset of chronic lipid oversupply by translocating to the cell surface and opening the cellular 'doors' for fatty acids. The sequence of events after the CD36-mediated myocellular lipid accumulation is less understood, but in general it has been accepted that the excessively imported lipids cause insulin resistance, which in turn leads to contractile dysfunction. There are several gaps of knowledge in this proposed order of events which this review aims to discuss. First, the molecular mechanisms underlying lipid-induced insulin resistance are not yet completely disclosed. Specifically, several mediators have been proposed, such as diacylglycerols, ceramides, peroxisome proliferator-activated receptors (PPAR), inflammatory kinases and reactive oxygen species (ROS), but their relative contributions to the onset of insulin resistance and their putatively synergistic actions are topics of controversy. Second, there are also pieces of evidence that lipids can induce contractile dysfunction independently of insulin resistance. Perhaps, a more integrative view is needed, in which several lipid-induced pathways operate synergistically or in parallel to induce contractile dysfunction. Unraveling of these processes is expected to be important in designing effective therapeutic strategies to protect the lipid-overloaded heart.
\end{abstract}




\section{Introduction}

ATP is required as a primary energy source to maintain the contractile function of the healthy heart. Long-chain fatty acids, together with glucose, present the major substrates for cardiac ATP production. For this, the fatty acids undergo $\beta$-oxidation in the mitochondria of the cardiomyocytes, yielding acetyl-CoA that enters the Krebs cycle to result in $\mathrm{NADH}$ and $\mathrm{FADH}_{2}$. These latter reduced coenzymes deliver their highenergy electrons to the electron transfer chain for the establishment of a mitochondrial proton gradient and the associated production of ATP $[1,2]$. Fatty acids have the advantage over the second important energy substrate glucose in that, when oxygen delivery is not limited, they provide more ATP per molecule and that they can be more optimally stored, namely as triacylglycerols. Yet, despite their crucial role in cardiac energy provision, fatty acids can initiate a chain of harmful events, especially when their supply continuously exceeds the metabolic demands of the heart. Such situation frequently arises upon sustained dietary lipid overconsumption as part of the Western lifestyle. Specifically, fatty acid oversupply gives rise to an imbalance between fatty acid uptake and oxidation, resulting in lipid accumulation in the cardiomyocytes [3]. Over time, lipid accumulation develops into a maladaptive state described as 'lipotoxicity', which refers to a condition in which lipids have accumulated to such levels that they become toxic to the cells. The latter implies a loss of contractile activity which is the main function of cardiomyocytes. At the level of the heart, there is decreased pump function, a state referred to as lipid-induced cardiomyopathy, which has been recognized as a major cause of death in type-2 diabetic patients.

The mechanisms by which lipids induce cardiac contractile dysfunction have been subject to intensive research, but until now remain only partly understood. However, it has become clear that myocellular lipid accumulation leads to the development of insulin resistance, which is defined as a metabolic state in which the cells are unable to respond properly to insulin (i.e., by increasing glucose uptake and accumulate glycogen). Subsequently, insulin resistance may lead to contractile dysfunction. There has been a debate on the possible molecular mechanisms by which lipids induce insulin resistance. Several possibilities have been described, including formation of 'toxic' lipid intermediates (diacylglycerols, ceramides), activation of peroxisome proliferator-activated receptors (PPARs), ROS production and the onset of inflammation. Surprisingly, little is known about the molecular mechanisms triggered by the insulin resistant state that result in an impairment of cardiac contractile function. On the other hand, lipids may cause contractile dysfunction independently of insulin resistance. Possible mechanisms for such lipid action may include effects on caveolins and/or direct sterical hindrance of the contractile apparatus. Most likely, in the heart (excess) lipids set in motion a pleiotropy 
of maladaptive actions some of which act through insulin resistance and some of which act in a different manner.

This review aims to provide an overview of possible mechanisms of lipid-induced insulin resistance and contractile dysfunction with an attempt to integrate insulinresistance-dependent and independent pathways within a scheme of lipid-induced contractile dysfunction. First, we will introduce the lipid metabolic pathways and insulin signaling kinases that are operative in the heart and subject to changes during lipid overload (sections 2 and 3). Then, in section 3, lipid-induced translocation of the fatty acid transporter CD36 is presented as the key early event in cardiac lipid oversupply and as causal factor in myocardial lipid accumulation. Finally, we will discuss in detail the suggested various mechanisms underlying lipid-induced contractile dysfunction (sections 4-6).

\section{Cardiac fatty acid metabolism}

Fatty acids provide $50-70 \%$ of the energy for cardiac contractile activity. The majority of fatty acids enter the cardiomyocytes via protein-mediated transport, whereas passive diffusion to a lesser extent contributes to bulk uptake of fatty acids [4]. The heart expresses various membrane-associated proteins facilitating fatty acid uptake and which, for convenience, often are designated as fatty acid transporters, including plasma membrane fatty acid-binding protein (FABPpm), CD36, fatty acid-transport protein-1 (FATP1), and FATP6. CD36 was observed to be responsible for the bulk uptake ( $>70 \%)$ of fatty acids, which finding was based on studies of substrate uptake kinetics in cardiomyocytres from CD36 knockout versus wild-type mice [5].

CD36 is an integral membrane protein with two membrane-spanning regions enclosing a large extracellular loop which contains the fatty acid-binding pocket [6]. CD36 not only is present at the sarcolemma, but also is stored intracellularly, i.e., within endosomal membranes. This endosomal storage is most prominent under conditions of a low demand for energy, whereby $\sim 50 \%$ of the CD36 population is present within endosomes [7]. Two major physiological stimuli, i.e., (i) increased metabolic demands (resulting from increased workload) and (ii) increased circulating insulin levels, induce the translocation of endosomal CD36 to the cell surface, leading to increased fatty acid uptake. Stimulation of CD36 translocation by increased metabolic demands requires activation of the cell's major energy sensor AMP-activated kinase (AMPK), while insulin-stimulated CD36 translocation requires activation of Akt2 (see section 3) [8]. CD36 translocation is a vesicle-mediated process, involving coat proteins for vesicle budding, Rab proteins for providing the unidirectionality in vesicle transport, and SNARE proteins for the specificity of trafficking [7]. Under non-stimulated (resting) conditions, Rab proteins are kept in an inactive state by the Rab-GTPase action of Akt 
substrate of $160 \mathrm{kDa}$ (AS160). Both Akt and AMPK inhibit AS160 via phosphorylation, leading to de-inhibition of the Rabs, and subsequent stimulation of CD36 translocation. In all these aspects, CD36 translocation closely resembles the well-characterized process of GLUT4 translocation, which is also stimulated by increased metabolic demands and insulin via activation of AMPK and Akt2, respectively, leading to AS160 phosphorylation and stimulation of glucose uptake [7].

Following their uptake into cardiomyocytes and subsequent transport through the cytoplasm by the small $(15 \mathrm{kDa})$ cytoplasmic heart-type fatty acid-binding protein $(\mathrm{FABP})$, fatty acids are rapidly converted into acyl-CoA esters by fatty acyl-CoA synthase (FACS) located at the outer mitochondrial membrane. In the heart, acyl-CoA esters have two major metabolic fates: mitochondrial $\beta$-oxidation and storage as triacylglycerol in lipid droplets.

In the healthy heart, fatty acids are primarily destined for mitochondrial $\beta$-oxidation, of which carnitine palmitoyl transferase 1 (CPT1) is the major gatekeeper. Under low energy demands CPT1 is kept in a relatively inactive state via its negative regulator malonyl-CoA. Increased metabolic demands activate CPT1 by inhibiting malonyl-CoA production via acetyl-CoA carboxylase (ACC). The underlying mechanism includes activation of AMPK, which phosphorylates and thereby inhibits ACC. CPT1 then converts acyl-CoA into acyl-L-carnitine. Given that AMPK also phosphorylates AS160 for stimulation of CD36 translocation, the coordinated phosphorylation of both AS160 and CPT1 ensures that under high energy demands increased fatty acid uptake is tightly linked to subsequent mitochondrial $\beta$-oxidation. Once inside the mitochondria, acyl-Lcarnitine is converted back to fatty acyl-CoA by carnitine palmitoyl transferase 2 (CPT2). Fatty acyl CoA then enters the $\beta$-oxidation pathway to produce acetyl CoA, NADH, and $\mathrm{FADH}_{2}[9,10]$. A schematic overview of fatty acid metabolism in cardiomyocytes is provided in Figure 1.

Excess fatty acyl-CoA, i.e., not passing CPT1 for mitochondrial $\beta$-oxidation, is converted into triacylglycerol in a stepwise fashion by consecutive actions of glycerol-3phosphate acyltransferase (GPAT), lipin, and diacylglycerol-acyltransferase (DGAT), rendering the process of triacylglycerol synthesis to serve as an overflow pathway for mitochondrial $\beta$-oxidation. Triacylglycerol is stored within a hydrophobic core of neutral lipid surrounded by a phospholipid monolayer and associated proteins, and the whole structure is referred to as lipid droplet. Among the lipid droplet-associated proteins are adipose triacylglycerol lipase (ATGL) and hormone-sensitive lipase (HSL) which each will mediate the liberation of fatty acid moieties from these droplets for mitochondrial $\beta$-oxidation when the energy requirements exceed the fatty acid supply [11]. 


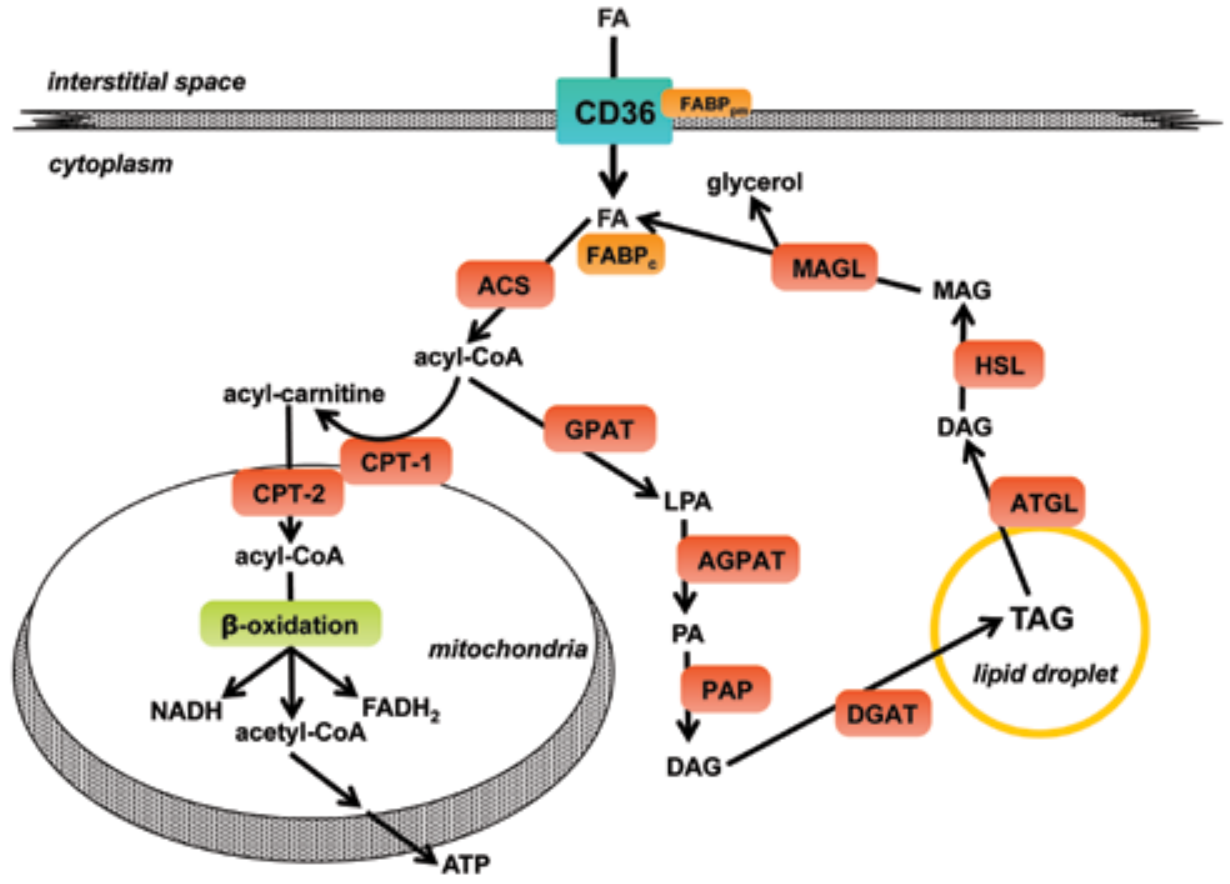

Figure 1. Fatty acid metabolism in cardiomyocytes. CD36, either alone or in collaboration with FABPpm and/or cytoplasmic FABP, facilitates long chain fatty acid (FA) uptake. ACS activates FA into acyl-CoA, which then is channelled either towards mitochondria or storage. CPT-1 catalyzes the formation of acyl-L-carnitine allowing for subsequent mitochondrial import by carnitine-acylcarnitine translocase (not shown). After reconversion by CPT-2 into acyl-CoA, the process of $\beta$-oxidation produces acetyl-CoA that enters the citric acid cycle and reducing equivalents $\left(\mathrm{NADH}, \mathrm{FADH}_{2}\right)$, together allowing the production of ATP in the respiratory chain (not shown). Excess cytosolic acylCoA is stored as TAG in lipid droplets via a series of enzymatic reactions producing LPA, PA and DAG as intermediates involving the enzymes GPAT, AGPAT, PAP, respectively, and DGAT. TAG can be hydrolyzed stepwise via DAG, MAG to produce FA and glycerol by ATGL, HSL and MAGL, respectively.

Abbreviations: ACS, acyl-CoA synthase; AGPAT, 1-acylglycerol-3-phosphate acyltransferase; ATGL, adipose triglyceride lipase; ATP, adenosine triphosphate; CPT-1, carnitine palmitoyl transferase 1; CPT-2, carnitine palmitoyl transferase 2; DAG, diacylglycerol; DGAT, diacylglycerol acyltransferase; FA, fatty acid; FABP, fatty acid binding protein; $\mathrm{FADH}_{2}$, reduced form of flavin adenine dinucleotide; GPAT, glycerol-3-phosphate acyltransferase; HSL, hormone-sensitive lipase; LPA, lysophosphatidic acid; NADH, reduced form of nicotinamide adenine dinucleotide; PA, phosphatidic acid; PAP, phosphatidate phosphatase-1; MAG, monoacylglycerol; MAGL, monoacylglycerol lipase; TAG, triacylglycerol.

\section{Insulin signaling in the heart and insulin resistance}

Insulin plays a key role in the regulation of substrate transport and utilization in the heart, by stimulating both cardiac glucose and fatty acid uptake via GLUT4 and CD36 translocation, respectively [7]. Insulin signal transduction in cardiomyocytes comprises a complex series of events initiated by activation of the insulin receptor (IR) tyrosine kinase, which is followed by tyrosine phosphorylation of insulin receptor substrates 1 
and -2 (IRS-1/2). IRS phosphorylation activates phosphatidylinositol 3-kinase (PI3K), the main player of the metabolic action of insulin. PI3K then associates with the plasma membrane, whereit phosphorylatesinositol phospholipidsto producephosphatidylinositol $(3,4,5)$-trisphosphate $\left(\mathrm{PIP}_{3}\right)$. The increase in $\mathrm{PIP}_{3}$ at the plasma membrane induces the recruitment and activation of the phosphoinositide-dependent kinase 1 (PDK1), which further phosphorylates and activates PKB/Akt [12]. Akt exists in three isoforms of which Akt2 regulates cardiac metabolism [13]. Akt is at the crossroads of many signaling pathways and has quite a number of cellular targets. For insulin-stimulated glucose uptake, Akt2's phosphorylation of AS160 is especially important [8], as mentioned in the previous section. Upon stimulation with insulin, AS160 is found to be phosphorylated on at least five sites, one of which is the 14-3-3 binding phospho-Thr649 in mice, as equivalent to Thr642 in human AS160 [14, 15]. Studies indicate that mice in which AS160-Thr649 is replaced by a non-phosphorylatable alanine residue are glucose intolerant, and show decreased insulin sensitivity and altered GLUT4 trafficking [15]. In parallel to $\mathrm{PKB} / \mathrm{Akt}$, PDK1 activates the atypical protein kinase $\mathrm{C}(\mathrm{PKC})$ isoforms $\lambda$ and $\zeta$, which contribute to the translocation of GLUT4, and hence stimulate glucose uptake $[16,17]$.

When lipid supply is chronically increased, either in vitro or in vivo, the ability of this canonical insulin signaling pathway to respond to insulin is drastically diminished. First, upon lipid oversupply, the delicate balance between CD36-mediated fatty acid uptake, mitochondrial $\beta$-oxidation, fatty acid storage and triacylglycerol hydrolysis, as present in the healthy heart, becomes challenged. When examining the time course of development of lipid-induced insulin resistance in vivo in rat skeletal muscle, a net translocation of CD36 from endosomes to the sarcolemma was one of the first changes observed (within 3 days after the start of the high fat diet regime) [18]. In heart and muscle, this CD36 translocation then causes a chronical increase in basal fatty acid uptake while at the same time insulin-stimulated fatty acid uptake is lost [18, 19] (Figure 2). This suggests that the CD36 pool that is stored in insulin-responsive endosomal subcompartments is responsible for the chronic increase in fatty acid uptake. Very rapidly after CD36 translocation and increased fatty acid uptake, i.e., within the same day, accumulation of lipid species (triacylglycerols, diacylglycerols and ceramides) in muscle was observed [18]. In contrast, changes in insulin signaling and insulin-stimulated glucose uptake occurred much later, i.e., after three weeks of high fat diet. Importantly, these latter changes are dependent on increased CD36 translocation, as CD36 ablation protects against high fat diet-induced insulin resistance [18]. Changes in mitochondrial parameters also occurred not until after three weeks [18]. Also in heart, a similar early increase in CD36 translocation was observed together with a loss in contractile function that followed much later (between 4 and 8 weeks) [19]. Hence, this rapid CD36 
translocation event is a key determinator of lipid-induced insulin resistance and contractile dysfunction. Moreover, the time lag between CD36 translocation and lipid accumulation on one hand, and insulin resistance and contractile dysfunction on the other, may suggest that the underlying molecular processes are time-consuming and complex (Figure 2).

In the remainder of this review we will present the various proposed mechanisms that may explain how lipids can give rise to cardiomyopathy and heart failure. As mentioned above, these lipid-triggered adaptations may include the development of insulin resistance, or alternatively may induce cardiomyopathy in a direct manner independent from insulin resistance. In the latter scenario, insulin resistance rather would be an innocent bystander or perhaps even a compensatory process. The mechanisms upstream of lipid-induced CD36 translocation are currently unknown and remain outside the scope of this review. First, we will discuss the possible routes from lipids to cardiomyopathy including insulin resistance. Then, we will discuss the possibility of lipid-induced cardiac dysfunction independent of insulin resistance. Finally we aim to present an integrative view of the various mechanisms involved.

\section{Mediators of lipid-induced insulin resistance}

When any of the kinases involved in the insulin signaling cascade is impaired, symptoms of insulin resistance would develop. Several lipid metabolites, especially diacylglycerols and ceramides, affect insulin signaling [20, 21]. During chronic lipid oversupply, all lipid metabolizing pathways become saturated so that the concentration of a great number of lipid metabolites increases. First, the combined actions of CD36 and FACS, i.e., coupling of fatty acid uptake to rapid conversion of fatty acids into acyl-CoA, ensure the maintenance of a steep fatty acid gradient from outside-to-inside the cell, and therefore a chronically increased fatty acid influx, which underlies all subsequent changes in fatty acid metabolism. Diacylglycerol will be generated in the triacylglycerol synthesis pathway by the phosphatidate phosphatase action of lipin, and in the triacylglycerol degradation route by the lipolytic actions of ATGL and HSL. Parts of the fatty acyl-CoA overshoot are covalently coupled to serine by serine-palmitoyltransferase leading to ceramide production. The most prominent hallmarks of lipid accumulation, the triacylglycerols stored in lipid droplets, are considered inert, and would not alter insulin signaling directly. Additionally, other factors, including PPARs, inflammatory molecules, and reactive oxygen species (ROS), have been proposed to mediate lipidinduced insulin resistance. The molecular mechanisms by which these factors may act, is discussed below in a pointwise manner. Yet, this list is not complete as various other possibilities, such as the roles of uncoupling proteins (at the most a minor contribution) and endoplasmic reticulum stress (more important in liver) are not discussed. 

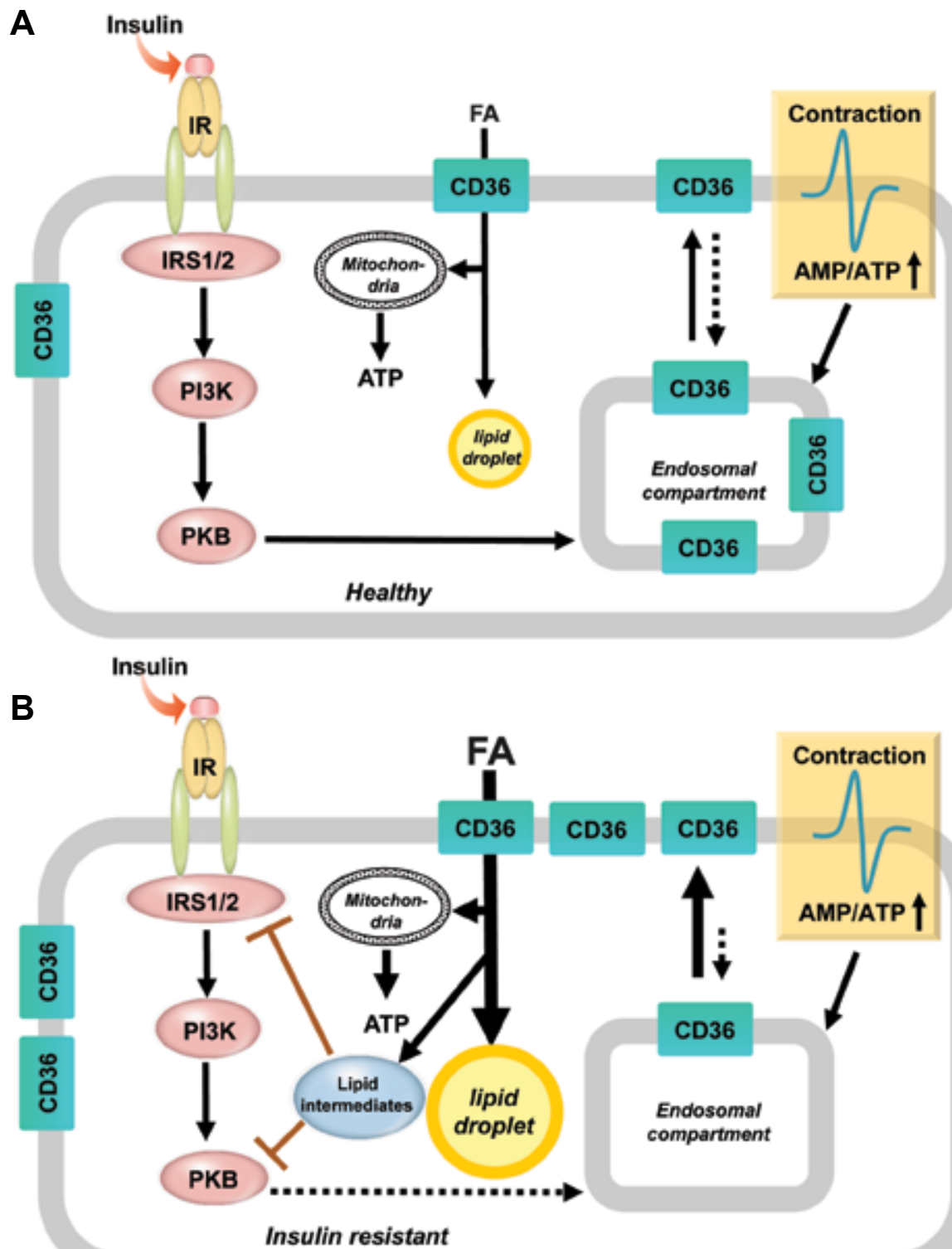

Figure 2. Crucial role of CD36 in lipid-induced insulin resistance. Fatty acid (FA) uptake by the healthy heart is primarily facilitated by fatty acid transporter CD36. Following insulin or contraction stimulation, CD36 translocates from endosomes to the sarcolemma, enhancing FA uptake. Upon lipid overload - through yet unknown mechanisms - CD36 becomes highly abundant at the cell surface. This net CD36 relocation to the sarcolemma results in enhanced FA uptake, increased TAG storage and increased formation of lipid intermediates which inhibit insulin signaling at the sites of IRS1/2 and $\mathrm{PKB} /$ Akt.

Abbreviations: AMP, adenosine monophosphate; ATP, adenosine triphosphate; CD36, fatty acid translocase FAT/CD36; IR, insulin receptor; IRS1/2, IR substrate 1/2; PI3K, phosphoinositide 3-kinase; $\mathrm{PKB}$, protein kinase B/Akt; TAG, triacylglycerol. 


\section{Diacylglycerol}

Diacylglycerol is known to be a potent activator of both conventional and novel PKC isoforms [22, 23] (Bronfman, 1988; Nishizuka Y, 1995). The PKC family consists of three subfamilies, based on their second messenger requirement: conventional ( $\alpha, \beta$ and $\gamma$ ), novel $(\delta, \varepsilon, \eta$, and $\theta)$, and atypical PKC's ( $\zeta$ and $\lambda)$ [23]. Conventional PKC activation is the result of binding of calcium to the $\mathrm{C} 2$ domain, increasing the interaction between the $\mathrm{C} 1$ domain and diacylglycerol, which in turn releases the pseudosubstrate inhibitory domain [24]. In contrast, novel PKCs inherently have much greater affinity for diacylglycerol while binding to the $\mathrm{C} 1$ domain [25]. Especially PKC- $\theta$ has been linked with diacylglycerol-mediated insulin resistance. PKC- $\theta$ causes serine/threonine phosphorylation of the insulin receptor [26] and of IRS-1 [20, 27, 28], thereby inhibiting the PI3K/PKB/Akt pathway which subsequently leads to insulin resistance [29]. The key role of diacylglycerol-mediated PKC- $\theta$ activation in the pathogenesis of insulin resistance has been confirmed in human muscle [30]. However, observations linking highpalmitate-induced PKC- $\theta$ activation and inhibition of insulin-stimulated GLUT4 translocation in heart and skeletal muscle are scarce. Specifically, it has been reported that in L6-muscle cells made insulin resistant by cytokines (which might play a role in lipid-induced insulin resistance, as detailed below in the subsection Inflammation) from conditioned media [31]. Pharmacological inhibition or silencing of novel PKCs restored insulin-stimulated GLUT4 translocation. However, in palmitate-treated cardiomyocytes that exhibited impaired insulin signaling and insulin-stimulated glucose uptake, diacylglycerol levels were not altered [32]. Taken together, there is conflicting evidence for a possible role of diacylglycerol/PKC- $\theta /$ IRS 1 axis in lipid-induced cardiac insulin resistance. These differences could be due to the use of in vitro models versus in vivo, on differences in regulation in heart versus muscle, and on differences in the species of fatty acids supplied.

\section{Ceramides}

The intracellular ceramide concentration is elevated in insulin resistant states of skeletal muscles, and therefore it has been claimed as a primary lipid mediator in muscle [33]. Several studies also documented such role of ceramides in cardiac insulin resistance $[34,35]$. Ceramide directly activates PKC $\zeta$, a member of the atypical PKCs, which phosphorylates and inhibits the membrane-attachment of Akt/PKB, thereby inhibiting insulin-stimulated glucose uptake by blocking glucose transporter GLUT4 translocation, as well as glycogen synthesis $[36,37]$. In high fat diet rodent models of insulin resistance, pharmacological inhibition of serine palmitoyltransferase enhances insulin action and insulin-stimulated glucose uptake in both muscle and heart [34, 38, 39]. Conversely, forced overexpression of sphingosine kinase, which prevents ceramide accumulation, 
ameliorates insulin resistance in high fat diet-fed mice [40]. Furthermore, cardiac overexpression of FACS, PPARa, PPAR $\gamma$, or FATP has been shown to elevate ceramide levels leading to dilated lipotoxic cardiomyopathy [41-44]. Human studies also confirm that total ceramide levels of skeletal muscle are increased nearly 2-fold in obese insulinresistant subjects, which is accompanied by a significant reduction in activated Akt/PKB levels [45]. Finally, myocardial ceramide levels were found to be decreased together with a reversal of insulin resistance in heart failure patients upon implantation of ventricular assist devices [46]. However, in a subset of rodent models of lipid-induced insulin resistance, the association between ceramide levels and insulin resistance was not observed [38].

\section{Peroxisome proliferator-activated receptors (PPARs)}

Myocardial fatty acid utilization is controlled, at least in part, at the gene regulatory level, especially during chronic adaptations. Evidence is emerging that nuclear receptors are involved in the development of lipid-induced insulin resistance. The peroxisome proliferator-activated receptors (PPARs) are a family of ligand-activated transcription factors that belong to the nuclear hormone receptor superfamily [47]. PPARs mainly exist in three subtypes: $\alpha, \beta / \delta$, and $\gamma$, which are expressed in various tissues in specific ratios and have diverse but overlapping functions in regulating lipid metabolism [48]. Yet, only $\alpha$ and $\beta / \delta$ isoforms regulate lipid metabolism in the heart [49]. PPARs bind fatty acids with a preference for long-chain fatty acids. In addition, numerous fatty acid metabolites, including acyl-CoAs, oxidized fatty acids, eicosanoids, etc., activate PPARs [50].

PPARa plays a central role in mediating the expression of proteins and enzymes involved in the physiological actions of mitochondrial $\beta$-oxidation of fatty acids, and, specifically, is known to be responsible for upregulating the expression of membraneassociated and cytoplasmic fatty acid transporters [51,52]. Cardiac-specific overexpression of PPAR $\alpha$ in mice results in enhanced fatty acid uptake, triacylglycerol accumulation, and reduced glucose-oxidation [53]. Additional studies indicate that cardiac-specific overexpression of PPARa in mice leads to cardiac insulin resistance associated with defects in insulin signaling, and subsequently to reduced cardiac function [54]. Interestingly, the absence of CD36 prevented cardiac triacylglycerol accumulation and cardiac dysfunction in the cardiac-restricted overexpression of PPAR $\alpha$ mice, which is associated with increased glucose utilization [55]. Conversely, PPAR $\alpha$-null mice display a decreased capacity of fatty acid metabolism, which likely contributes to dyslipidemia $[56,57]$. Interestingly, PPARa-null mice are protected from the development of insulin resistance when feeding with a high fat diet [58]. Yet, the beneficial effects of inhibition of fatty acid oxidation via PPARa downregulation are difficult to comprehend when 
given that the fatty acid excess would overspill the other lipid metabolic pathways [59]. Likely, the absence of PPAR $\alpha$ reduces the rate of fatty acid oxidation, whereas the utilization of glucose is enhanced despite lower glucose availability in high fat diet-fed mice. Accordingly, PPARa agonists were proposed and used to restore metabolism in the diabetic heart [59]. However, the PPARa ligand BM17.0744 did not restore cardiac function in diabetic mice [60]. In a broader sense, the cardio-lipotoxic actions of PPAR overexpression do not match with the proposed beneficial effects of PPARa ligands. Furthermore, PPAR $\alpha$ and PPAR $\beta / \delta$ may have overlapping targets and function in the heart. Cardiac-specific $\mathrm{PPAR} \beta / \delta$ overexpressing transgenic mice do not display myocardial lipid accumulation or cardiac dysfunction, even on high fat diet [61]. Taken together, there is much controversy whether PPARs are friends or foes in lipid-induced cardiomyopathy [62].

\section{Reactive oxygen species (ROS)}

ROS are generated as by-product of mitochondrial $\beta$-oxidation as a result of some leakage of electrons to oxygen $\left(\mathrm{O}_{2}\right)$ at several sites of the electron transport chain to form superoxide. Antioxidant systems convert superoxide to hydrogen peroxide $\left(\mathrm{H}_{2} \mathrm{O}_{2}\right)$, which is then further degraded into water by catalase. In numerous rodent models of excessive fat feeding and insulin resistance, fatty acid oxidation is increased as a result of the increased fatty acid supply $[19,63,64]$. The resulting increased flux through the electron transport chain is likely to cause increased ROS formation and $\mathrm{H}_{2} \mathrm{O}_{2}$ generation $[64,65]$. Persistent up-regulation of fatty acid oxidation leads to chronically increased ROS formation, which may exhaust the several cellular anti-oxidant systems. Because mitochondrial membranes are especially vulnerable to damage by ROS, progressive mitochondrial damage occurs, ultimately resulting in a decreased fatty acid oxidation capacity. Thus, decreased fatty acid oxidation inevitably follows sustained periods of increased fatty acid oxidation. Therefore, the conflicting literature on whether myocardial fatty acid oxidation is increased [64,65] versus decreased [66-68] in rodent models of insulin resistance might be a matter of timing. The decrease in mitochondrial capacity and the resulting decrease in the cardiac energy state could be responsible for cardiac dysfunction in insulin resistant rodents, but this has not yet been fully explored [65]. Furthermore, decreased mitochondrial flux redirects the fatty acids to lipid storage pathways, which may induce a vicious cycle of further accumulation of diacylglycerols, ceramides and triacylglycerols.

While mitochondria are the major source of ROS, also enhanced extra-mitochondrial ROS production might contribute to ROS accumulation in the insulin resistant heart. Indeed, the activity of NADPH oxidase (NOX), the major source of extra-mitochondrial ROS, is elevated in hearts of obese Zucker rats, ob/ob mice, and high fat diet-fed rats [69, 
70]. Interestingly, pharmacological NOX inhibition reversed cardiac dysfunction in several insulin resistant rodent models $[70,71]$. Thus, cytosolic ROS production may play a role in inducing mitochondrial damage, thereby further impairing mitochondrial function, and directly leading to decreased cardiac contraction [72].

\section{Inflammation}

Inflammation has been directly and indirectly associated with lipid-induced insulin resistance and has become a prevailing view to explain the pathogenesis of type 2 diabetes. The fact that inflammation might be one of the contributors to insulin resistance is based on the observation that anti-inflammatory drugs like salicylates reduce hyperglycemia in diabetic patients [73]. Various studies have demonstrated that obesity and high fat diet-feeding causes the recruitment of macrophages, not only to adipose tissue, but also to the heart, concomitant with the production of proinflammatory cytokines, such as tumor necrosis factor- $\alpha$ (TNF- $\alpha$ ) and interleukin-6 (IL-6) [74, 75]. Additionally, cardiomyocytes, like many other mammalian cells, produce these cytokines upon direct exposure to fatty acids via fatty acid binding to the patternrecognition receptor, Toll-like receptor-4 (TLR4) [76]. TLRs are essential for mounting inflammatory responses associated with innate immunity, but are also activated in the presence of chronically activated fatty acid concentrations [77]. TLR activation, through MyD88, results in activation of I $\mathrm{B}$ kinase (IKK), movement of the nuclear factor- $\kappa \mathrm{B}$ to the nucleus leading to cytokine production $[78,79]$. These cytokines, TNF- $\alpha$ and IL- 6 , on their turn, through binding to their receptors in an autocrine amplification loop, induce Ser-phosphorylation of IRS via suppressor of cytokine signaling-3 (SOCS-3) and c-Jun $\mathrm{N}$-terminal kinase (JNK) [80]. Moreover, fatty acid activation of TLR4 also directly activates JNK and PKCs, and thereby induce Ser/Thr-phosphorylation of IRS [81]. As mentioned in section 3, this Ser/Thr-phosphorylation of IRS will lead to insulin resistance.

Interestingly, lipid-induced insulin resistance in muscle mediated by TLR4 requires the generation of ceramide [82]. TLR4-stimulated ceramide generation is postulated to be mediated by IKK activation and the synthesis of enzymes in the ceramide synthesis pathway (e.g., serine palmitoyltransferase). Hence, the inflammatory pathways have a closely intertwined relationship with the lipid-induced signaling pathways to induce insulin resistance [83].

\section{Insulin resistance and subsequent cardiac contractile dysfunction}

Both rodent models and clinical studies support that lipid-induced insulin resistance eventually precipitates into cardiac contractile dysfunction [84]. Also in vitro, in cardiomyocyte cultures exposed to excess fatty acids, the onset of insulin resistance 
occurred in association with a marked decrease in contractile activity of the cardiomyocytes [32]. However, the relationship between insulin resistance and cardiac contractile function remains unclear. Potential mechanisms may include abnormal intracellular $\mathrm{Ca}^{2+}$ dynamics or myosin isozyme switch, as discussed below [85-87].

\section{$C a^{2+}$ dynamics}

Myocardial contractile force is dependent on the intracellular $\mathrm{Ca}^{2+}$ concentration and its regulation, because both the release and the re-uptake of intracellular $\mathrm{Ca}^{2+}$ are necessary to maintain the normal systolic and diastolic functions of the heart. Therefore, changing the availability of $\mathrm{Ca}^{2+}$ to the myofilaments or shifting the responsiveness of the myofilaments to activation by intracellular $\mathrm{Ca}^{2+}$ may alter cardiac contractile function. It has been observed that in the failing heart the density of $\mathrm{Ca}^{2+}$ uptake sites on the sarcoplasmic reticulum is decreased, and that $\mathrm{Ca}^{2+}$ release is impaired. Thus, abnormal handling of intracellular $\mathrm{Ca}^{2+}$ may be a primary cause of contractile dysfunction $[88,89]$. Sarcoplasmic reticulum $\mathrm{Ca}^{2+}$ storage is possibly reduced in diabetic cardiomyocytes, yet the mechanism remains unknown [90]. In cardiomyocytes from diet-induced insulin resistant rats, decreased SERCA activity contributed to contractile dysfunction of insulin resistant cardiomyocytes [91]. Furthermore, altered cardiomyocyte $\mathrm{Ca}^{2+}$ handling appears to gain importance in the later stages of diabetes [91].

\section{Myosin isozyme switch}

ATP hydrolysis by myofibrillar and myosin ATPase is the rate-limiting step in of cardiac contraction. It is generally accepted that reduced myofibrillar ATPase activity is associated with contractile dysfunction during the onset of insulin resistance [84]. The underlying mechanism for decreased activity of this ATPase is a switch in myosin isozyme expression, i.e., from myosin heavy chain (MHC)- $\alpha / \alpha$ homodimer to the $\beta / \beta$ homodimer $[92,93]$. This increased $\beta$-MHC expression slows actin-myosin kinetics, and contributes to contractile dysfunction of the insulin resistant rodent heart [94]. The shift to the less efficient $\beta$-MHC in the insulin resistant heart also causes a marked reduction in formation and dissociation of actin-myosin cross-bridges [94]. Because this crossbridge cycling is important in force development, cross-bridge dysregulation could contribute to contractile dysfunction.

Several other contractile derangements could contribute to contractile dysfunction in the insulin resistant heart, such as changes in accessory proteins of the thick filament, in the actin thin filament or in the extra-sarcomeric cytoskeleton, but some of these changes are still controversial and need further investigation [84]. Taken together, the exact mechanism how cardiac insulin resistance causes contractile dysfunction is not known yet. 
Interestingly, rodent studies indicate that contractile dysfunction of cardiomyocytes from insulin resistant rats can be prevented by metformin treatment [85]. Metformin likely does not directly impact on the heart, but will act in an indirect manner via reducing hepatic gluconeogenesis. Moreover, metformin treatment normalizes mechanical indexes, suggesting that cardiac contractile dysfunction can be prevented by inhibition of insulin resistance [85].

\section{Impairment of cardiac contractile function by lipids independently from insulin resistance}

Although it is evident that lipid excess over time causes cardiac insulin resistance, and that cardiac insulin resistance may further progress into contractile dysfunction, there are also several indications that lipids can worsen cardiac function independently of insulin resistance. This line of evidence is derived from studies with transgenic mouse models with altered contents of lipid metabolizing enzymes, i.e., allowing for separation of lipid accumulation from insulin resistance. In mice with cardiac overexpression of DGAT, the rate limiting enzyme in the triacylglycerol synthesis, diacylglycerol levels were unchanged, while triacylglycerol levels were increased. These mice exhibited no metabolic derangements including changes in insulin sensitivity, but yet displayed diastolic dysfunction and cardiomyopathy [95]. However, these findings contrast with another study, in which cardiospecific DGAT overexpression did not lead to cardiomyopathy, but rather offered cardio-protection in a mouse model of cardiac lipid overload [96]. Additionally, ATGL knockout mice were studied to investigate the impact of triacylglycerol accumulation on cardiac function, and were found to exhibit severe systolic and diastolic dysfunction [97]. Yet, ATGL-driven lipid accumulation rather increased insulin sensitivity in heart and liver [98]. This cardiotoxic action of ATGL overexpression was attributed to diminished PPARa signaling [97], but this has been disputed [99]. Vice versa, cardio-specific ATGL overexpression prevents cardiac triacylglycerol accumulation and preserves cardiac function in mice on high fat diet, but does not alter insulin sensitivity [100]. Hence, these studies suggest that triacylglycerols, considered as an "inert" lipid species (i.e., without signaling actions), would induce cardiomyopathy via a different route. Also other lipid species, such as ceramides and acyl-carnitines might induce contractile dysfunction independently of insulin resistance, as discussed below. Taken together, this implies that therapeutic treatment strategies to combat lipid-induced cardiomyopathy via restoration of insulin signaling may not always be effective. Below, we will discuss some possible mechanisms of triacylglycerolmediated cardiomyopathy independent of insulin resistance. 


\section{Loss of caveolin-3}

A possible novel mechanism for lipid-induced cardiac contractile dysfunction includes the loss of caveolin-3 during cardiac lipid oversupply [101]. Caveolins are the defining protein constituents of caveolae, which are responsible for the invagination of the plasma membrane. Three members have been identified, and caveolin- 3 is the predominant isoform in muscle and heart. Caveolins play an important role in endocytosis, lipid trafficking, and signal transduction [102]. Caveolin-3 is also present in T-tubules and is associated with the development of the T-tubule system. Concordingly, caveolin-3 null mice have abnormal T-tubuli and display a phenotype similar to muscular dystrophy $[103,104]$. Loss of cardiac caveolin-3 or caveolin-3 mutations have been proven to induce hypertrophy that eventually leads to dilated cardiomyopathy [105-107]. High fat diet induces a loss of caveolin-3 together with contractile dysfunction [101]. This high fat diet-induced cardiomyopathy resembles the cardiomyopathy in the caveolin-3 null mice [104]. The mechanism is related with the downregulation of RyR in cardiomyocytes following the loss of T-tubule structure, and is expected to cause impaired calcium handling and contractile dysfunction. This novel finding suggests that the expression and intracellular localization of caveolin-3 is crucial in heart failure and could be a potential therapeutic target for the improvement of lipid-induced cardiac contractile dysfunction. Yet caveolin-3 deletion also impairs insulin signaling, as caveolae are important for functioning of insulin receptors [108]. Hence, it is difficult to study the role defective T-tubuli in the development of cardiac contractile dysfunction in caveolin-3 null mice separately from changes in insulin signaling.

\section{Acyl-carnitines}

During lipid overload, acyl-carnitines levels rise due to the high rates of fatty acid oxidation exceeding the metabolic flux through the tricaboxylic acid cycle [109]. Several mechanisms appear responsible for the pro-arrhythmic effects of acyl-carnitines. First, reduction of the single-channel conductance of the inward-going rectifier $\mathrm{K}^{+}$current may produce automatic action potential discharges resulting in ventricular tachycardia. Second, the decrease of the excitatory $\mathrm{Na}^{+}$current could generate conduction anomalies and result in re-entry [110]. Finally, amphipathic metabolites may impair gap-junction channels [111]. The increased risk of arrhythmias of the lipid-overloaded heart might contribute to the pathogenesis of cardiomyopathy.

\section{Apoptosis}

Apoptosis in the heart inevitably leads to decreased cardiac muscle mass, which may therefore contribute to loss of cardiac contractility. Lipids are known to induce apoptosis [112], and lipid-induced apoptosis contributes to the pleiotropy of cellular maladaptive 
actions of lipids, summarized in the term lipotoxicity (see section 1). Lipid-induced apoptosis is generally associated with excess ceramides [112-114]. Ceramides have been reported to increase expression of inducible nitric oxide synthase (iNOS), thereby increasing NO production, and consequently ROS production and ROS-dependent apoptosis [112]. Ceramides also activate JNK, which interacts with Bax in the mitochondrial membrane, causing release of cytochrome $\mathrm{c}$ and activation of the caspase cascade as part of the apoptotic pathway [113]. Given that these ceramide actions overlap with the effects of ceramides on inhibition of insulin signaling and activation of ROS and inflammation, it is likely that apoptosis induced by excess lipids/ceramides cannot be seen entirely independent of the onset of insulin resistance.

\section{Stereological hindrance}

Triacylglycerols stored in lipid droplets might directly impair cardiac contractility. As shown in electron-microscopical pictures from skeletal muscle of obese Zucker rats (a rat model of lipid oversupply), excess lipids primarily accumulate within myocytes in the form of lipid droplets in the intra-myofibrillar regions [115]. This interspersion of myofibrils with large lipid droplets might also be present in cardiomyocytes from rodent models of lipid oversupply. Perhaps, a simple explanation of lipid-induced contractile dysfunction could include that lipid droplets impair proper contraction mechanics just by spatial hindrance.

\section{Concluding remarks}

The putative mechanisms leading to lipid-induced insulin resistance and contractile dysfunction in the heart are manifold and together form a complex entity. Intentionally, we discussed these putative mechanisms separately, as summarized in the flow chart (Figure 3). However, when focusing first on the causes of insulin resistance (Figure 3, orange boxes), most of the concerned pathways are known to interact. For instance, sustained ROS production as a result of chronically increased fatty acid oxidation results in mitochondrial damage, which inevitably merges into a next phase of decreased fatty acid oxidation. This would then divert the incoming lipids increasingly into lipid storage pathways with the accompanying rise of diacylglycerols and ceramides. In this way an amplification loop is initiated. Another example relates to the interaction between inflammation, ROS and ceramides, where lipid-induced TLR4-dependent inflammatory signaling via the IKK/NF- $\kappa$ B route (and/or via TNF- $\alpha$ ) leads to JNK and PKC activation, ultimately causing inhibition of insulin signaling by serine phosphorylation of IRS [81]. Such inflammatory mechanism of decreased insulin sensitivity may thus amplify the direct effects of lipid intermediates (i.e., ceramides and diacylglycerols) on inhibition of insulin signaling. Furthermore, ROS [116] and ceramides [113] have been shown to activate the IKK/NF- $\kappa \mathrm{B}$ axis of inflammation signaling. Hence, ROS may impact on 


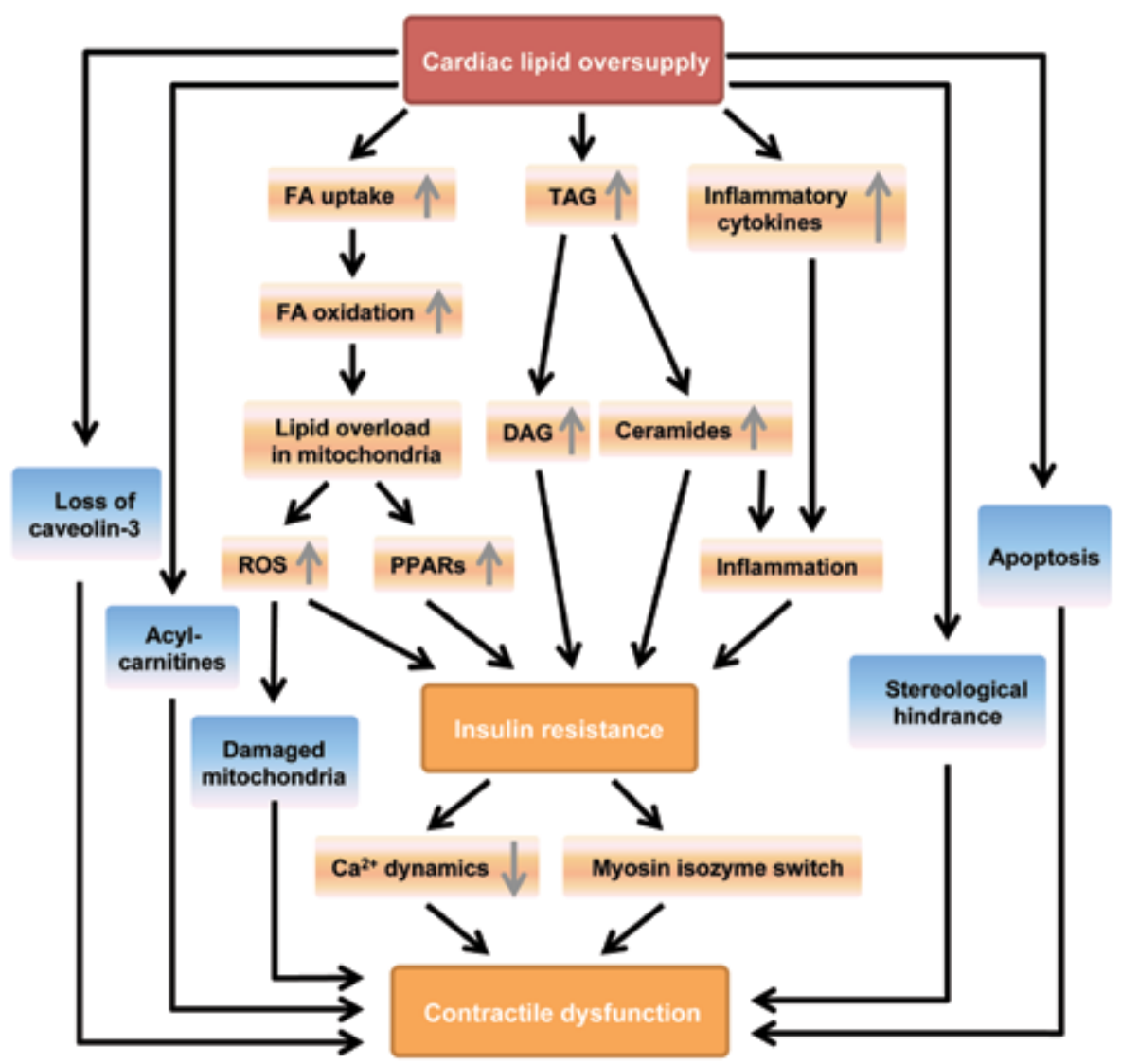

Figure 3. Putative pathophysiological mechanisms linking cardiac lipid oversupply to insulin resistance and contractile dysfunction. Upon lipid oversupply to the heart, mitochondrial $\beta$-oxidation of FA is initially increased, which in turn, leads to an increased oxidative stress and ROS production, as well as activation of PPARs, which each alone or together may be causal for insulin resistance. The increased ROS production may damage mitochondrial function, which directly leads to cardiac contractile dysfunction, or may further induce accumulation of TAG and the lipid intermediates DAG and ceramide. Excess lipids also directly induce the accumulation of TAG, DAG and ceramide, which may cause apoptosis, or attenuate insulin signaling either directly or via triggering of inflammatory signaling. In addition, activation of inflammatory cytokines by lipid accumulation might induce insulin resistance. Cardiac insulin resistance may further progress into contractile dysfunction via a decrease of $\mathrm{Ca}^{2+}$ dynamics or via a myosin isozyme switch. On the other hand, lipid accumulation may cause contractile dysfunction independent of cardiac insulin resistance via loss of caveolin-3, increased levels of acyl-carnitines, damaged mitochondrial function, apoptosis, or stereological hindrance.

insulin resistance via mitochondrial damage and inflammation (and perhaps also other processes), while ceramides may induce insulin resistance via Akt inhibition, ROS generation and by activation of pro-inflammatory signaling.

The picture gets even more complicated when also considering the insulin resistanceindependent mediators (as mentioned in section 6) in the pathogenesis of lipid-induced 
cardiomyopathy (Figure 3, blue boxes). This especially applies to apoptosis, which can be induced by either ROS, inflammation and/or ceramides (being classified in this review as insulin-dependent mediators of lipid-induced contractile dysfunction) via activation of the caspase pathway, more or less independently of insulin resistance [113, 114], and thereby further contribute to contractile dysfunction. In contrast, triacylglycerols (via stereological hindrance) and acyl-carnitines (via their pro-arrhythmogenic actions) appear to have little cross-talk with other mediators, and thus may contribute rather independently to decreased contractility in the lipid-overloaded heart.

Taken together, the mediators of lipid-induced contractile dysfunction discussed here interact in a complicated and partly overlapping manner, while various amplifying loops may contribute to further complexity. Several of these lipid-induced derangements were originally established in transgenic and diet-induced rodent models, and have been verified in humans [30, 117]. Further progress in this field is needed to serve the identification of new therapeutic targets and treatment strategies in order to combat lipid-induced cardiomyopathy. Yet, the most obvious strategy for humans is to abstain from chronic lipid overconsumption, which would prevent the onset of this major cause of cardiomyopathy.

\section{Acknowledgements}

Y.Liu was supported by a grant from the China Scholarship Council. D. Neumann is the recipient of a VIDI-Innovational Research grant from the Netherlands Organization for Scientific Research (NWO-ALW). 


\section{References}

1. J.S. Ingwall, Energy metabolism in heart failure and remodelling, Cardiovasc Res, 81 (2009) 412419.

2. W.C. Stanley, F.A. Recchia, G.D. Lopaschuk, Myocardial substrate metabolism in the normal and failing heart, Physiol Rev, 85 (2005) 10931129.

3. T.R. Koves, J.R. Ussher, R.C. Noland, D. Slentz, M. Mosedale, O. Ilkayeva, J. Bain, R. Stevens, J.R. Dyck, C.B. Newgard, G.D. Lopaschuk, D.M. Muoio, Mitochondrial overload and incomplete fatty acid oxidation contribute to skeletal muscle insulin resistance, Cell Metab, 7 (2008) 45-56.

4. J.F. Glatz, J.J. Luiken, A. Bonen, Membrane fatty acid transporters as regulators of lipid metabolism: implications for metabolic disease, Physiol Rev, 90 (2010) 367-417.

5. D.D. Habets, W.A. Coumans, P.J. Voshol, M.A. den Boer, M. Febbraio, A. Bonen, J.F. Glatz, J.J. Luiken, AMPK-mediated increase in myocardial long-chain fatty acid uptake critically depends on sarcolemmal CD36, Biochem Biophys Res Commun, 355 (2007) 204-210.

6. N.A. Abumrad, CD36 may determine our desire for dietary fats, J Clin Invest, 115 (2005) 29652967.

7. L.K. Steinbusch, R.W. Schwenk, D.M. Ouwens, M. Diamant, J.F. Glatz, J.J. Luiken, Subcellular trafficking of the substrate transporters GLUT4 and CD36 in cardiomyocytes, Cell Mol Life Sci, 68 (2011) 2525-2538.

8. H.K. Karlsson, J.R. Zierath, S. Kane, A. Krook, G.E. Lienhard, H. Wallberg-Henriksson, Insulin-stimulated phosphorylation of the Akt substrate AS160 is impaired in skeletal muscle of type 2 diabetic subjects, Diabetes, 54 (2005) 1692-1697.

9. J.D. McGarry, G.F. Leatherman, D.W. Foster, Carnitine palmitoyltransferase I. The site of inhibition of hepatic fatty acid oxidation by malonylCoA, J Biol Chem, 253 (1978) 4128-4136.

10. J.D. McGarry, N.F. Brown, The mitochondrial carnitine palmitoyltransferase system. From concept to molecular analysis, Eur J Biochem, 244 (1997) 1-14.

11. K. Jaworski, E. Sarkadi-Nagy, R.E. Duncan, M. Ahmadian, H.S. Sul, Regulation of triglyceride metabolism. IV. Hormonal regulation of lipolysis in adipose tissue, Am J Physiol Gastrointest Liver Physiol, 293 (2007) G1-4.

12. L. Bertrand, S. Horman, C. Beauloye, J.L. Vanoverschelde, Insulin signalling in the heart, Cardiovasc Res, 79 (2008) 238-248.

13. B. DeBosch, N. Sambandam, C. Weinheimer, M. Courtois, A.J. Muslin, Akt2 regulates cardiac metabolism and cardiomyocyte survival, J Biol Chem, 281 (2006) 32841-32851.

14. K.M. Geraghty, S. Chen, J.E. Harthill, A.F. Ibrahim, R. Toth, N.A. Morrice, F. Vandermoere, G.B. Moorhead, D.G. Hardie, C. MacKintosh, Regulation of multisite phosphorylation and 143-3 binding of AS160 in response to IGF-1, EGF, PMA and AICAR, Biochem J, 407 (2007) 231241.

15. S. Chen, D.H. Wasserman, C. MacKintosh, K. Sakamoto, Mice with AS160/TBC1D4-Thr649Ala knockin mutation are glucose intolerant with reduced insulin sensitivity and altered GLUT4 trafficking, Cell Metab, 13 (2011) 68-79.

16. M.L. Standaert, G. Bandyopadhyay, L. Perez, D. Price, L. Galloway, A. Poklepovic, M.P. Sajan, V. Cenni, A. Sirri, J. Moscat, A. Toker, R.V. Farese, Insulin activates protein kinases C-zeta and Clambda by an autophosphorylation-dependent mechanism and stimulates their translocation to GLUT4 vesicles and other membrane fractions in rat adipocytes, J Biol Chem, 274 (1999) 2530825316.

17. D.D. Habets, J.J. Luiken, M. Ouwens, W.A. Coumans, M. Vergouwe, S.J. Maarbjerg, M. Leitges, A. Bonen, E.A. Richter, J.F. Glatz, Involvement of atypical protein kinase $\mathrm{C}$ in the regulation of cardiac glucose and long-chain fatty acid uptake, Front Physiol, 3 (2012) 361.

18. A. Bonen, S.S. Jain, L.A. Snook, X.X. Han, Y. Yoshida, K.H. Buddo, J.S. Lally, E.D. Pask, S. Paglialunga, M.S. Beaudoin, J.F. Glatz, J.J. Luiken, E. Harasim, D.C. Wright, A. Chabowski, G.P. Holloway, Extremely rapid increase in fatty acid transport and intramyocellular lipid accumulation but markedly delayed insulin resistance after high fat feeding in rats, Diabetologia, 58 (2015) 2381-2391.

19. D.M. Ouwens, M. Diamant, M. Fodor, D.D. Habets, M.M. Pelsers, M. El Hasnaoui, Z.C. Dang, C.E. van den Brom, R. Vlasblom, A. Rietdijk, C. Boer, S.L. Coort, J.F. Glatz, J.J. Luiken, Cardiac contractile dysfunction in insulin-resistant rats fed a high-fat diet is associated with elevated CD36-mediated fatty acid uptake and esterification, Diabetologia, 50 (2007) 1938-1948.

20. C. Yu, Y. Chen, G.W. Cline, D. Zhang, H. Zong, Y. Wang, R. Bergeron, J.K. Kim, S.W. Cushman, G.J. Cooney, B. Atcheson, M.F. White, E.W. Kraegen, G.I. Shulman, Mechanism by which fatty acids inhibit insulin activation of insulin receptor substrate-1 (IRS-1)-associated phosphatidylinositol 3-kinase activity in muscle, $J$ Biol Chem, 277 (2002) 50230-50236. 
21. W.L. Holland, J.T. Brozinick, L.P. Wang, E.D. Hawkins, K.M. Sargent, Y. Liu, K. Narra, K.L. Hoehn, T.A. Knotts, A. Siesky, D.H. Nelson, S.K. Karathanasis, G.K. Fontenot, M.J. Birnbaum, S.A. Summers, Inhibition of ceramide synthesis ameliorates glucocorticoid-, saturated-fat-, and obesity-induced insulin resistance, Cell Metab, 5 (2007) 167-179.

22. M. Bronfman, M.N. Morales, A. Orellana, Diacylglycerol activation of protein kinase $C$ is modulated by long-chain acyl-CoA, Biochem Biophys Res Commun, 152 (1988) 987-992.

23. Y. Nishizuka, Protein kinase $\mathrm{C}$ and lipid signaling for sustained cellular responses, FASEB J, 9 (1995) 484-496.

24. A.C. Newton, Protein kinase C. Seeing two domains, Curr Biol, 5 (1995) 973-976.

25. D.R. Dries, L.L. Gallegos, A.C. Newton, A single residue in the $\mathrm{C} 1$ domain sensitizes novel protein kinase $\mathrm{C}$ isoforms to cellular diacylglycerol production, J Biol Chem, 282 (2007) 826-830.

26. S.I. Itani, Q. Zhou, W.J. Pories, K.G. MacDonald, G.L. Dohm, Involvement of protein kinase C in human skeletal muscle insulin resistance and obesity, Diabetes, 49 (2000) 1353-1358.

27. V.T. Samuel, G.I. Shulman, Mechanisms for insulin resistance: common threads and missing links, Cell, 148 (2012) 852-871.

28. L.V. Ravichandran, D.L. Esposito, J. Chen, M.J. Quon, Protein kinase C-zeta phosphorylates insulin receptor substrate- 1 and impairs its ability to activate phosphatidylinositol 3-kinase in response to insulin, J Biol Chem, 276 (2001) 35433549.

29. D.B. Savage, K.F. Petersen, G.I. Shulman, Mechanisms of insulin resistance in humans and possible links with inflammation, Hypertension, 45 (2005) 828-833.

30. J. Szendroedi, T. Yoshimura, E. Phielix, C. Koliaki, M. Marcucci, D. Zhang, T. Jelenik, J. Muller, C. Herder, P. Nowotny, G.I. Shulman, M. Roden, Role of diacylglycerol activation of PKCtheta in lipid-induced muscle insulin resistance in humans, Proc Natl Acad Sci U S A, 111 (2014) 9597-9602.

31. G. Kewalramani, L.N. Fink, F. Asadi, A. Klip, Palmitate-activated macrophages confer insulin resistance to muscle cells by a mechanism involving protein kinase $\mathrm{C}$ theta and epsilon, PLoS One, 6 (2011) e26947.

32. Y. Angin, L.K. Steinbusch, P.J. Simons, S. Greulich, N.T. Hoebers, K. Douma, M.A. van Zandvoort, W.A. Coumans, W. Wijnen, M. Diamant, D.M. Ouwens, J.F. Glatz, J.J. Luiken, CD36 inhibition prevents lipid accumulation and contractile dysfunction in rat cardiomyocytes, Biochem J, 448 (2012) 43-53.
33. M.J. Watt, N. Dzamko, W.G. Thomas, S. RoseJohn, M. Ernst, D. Carling, B.E. Kemp, M.A. Febbraio, G.R. Steinberg, CNTF reverses obesityinduced insulin resistance by activating skeletal muscle AMPK, Nat Med, 12 (2006) 541-548.

34. J.R. Ussher, C.D. Folmes, W. Keung, N. Fillmore, J.S. Jaswal, V.J. Cadete, D.L. Beker, V.H. Lam, L. Zhang, G.D. Lopaschuk, Inhibition of serine palmitoyl transferase I reduces cardiac ceramide levels and increases glycolysis rates following diet-induced insulin resistance, PLoS One, 7 (2012) e37703.

35. E. Ramirez, M. Klett-Mingo, S. Ares-Carrasco, B. Picatoste, A. Ferrarini, F.J. Ruperez, A. Caro-Vadillo, C. Barbas, J. Egido, J. Tunon, O. Lorenzo, Eplerenone attenuated cardiac steatosis, apoptosis and diastolic dysfunction in experimental type-II diabetes, Cardiovasc Diabetol, 12 (2013) 172.

36. E. Hajduch, A. Balendran, I.H. Batty, G.J. Litherland, A.S. Blair, C.P. Downes, H.S. Hundal, Ceramide impairs the insulin-dependent membrane recruitment of protein kinase B leading to a loss in downstream signalling in L6 skeletal muscle cells, Diabetologia, 44 (2001) 173-183.

37. S. Stratford, D.B. DeWald, S.A. Summers, Ceramide dissociates 3'-phosphoinositide production from pleckstrin homology domain translocation, Biochem J, 354 (2001) 359-368.

38. W.L. Holland, S.A. Summers, Sphingolipids, insulin resistance, and metabolic disease: new insights from in vivo manipulation of sphingolipid metabolism, Endocr Rev, 29 (2008) 381-402.

39. G. Frangioudakis, J. Garrard, K. Raddatz, J.L. Nadler, T.W. Mitchell, C. Schmitz-Peiffer, Saturated- and n-6 polyunsaturated-fat diets each induce ceramide accumulation in mouse skeletal muscle: reversal and improvement of glucose tolerance by lipid metabolism inhibitors, Endocrinology, 151 (2010) 4187-4196.

40. C.R. Bruce, S. Risis, J.R. Babb, C. Yang, G.M. Kowalski, A. Selathurai, R.S. Lee-Young, J.M. Weir, K. Yoshioka, Y. Takuwa, P.J. Meikle, S.M. Pitson, M.A. Febbraio, Overexpression of sphingosine kinase 1 prevents ceramide accumulation and ameliorates muscle insulin resistance in high-fat diet-fed mice, Diabetes, 61 (2012) 31483155 .

41. H.C. Chiu, A. Kovacs, D.A. Ford, F.F. Hsu, R. Garcia, P. Herrero, J.E. Saffitz, J.E. Schaffer, A novel mouse model of lipotoxic cardiomyopathy, J Clin Invest, 107 (2001) 813-822.

42. B.N. Finck, X. Han, M. Courtois, F. Aimond, J.M. Nerbonne, A.Kovacs, R.W.Gross, D.P.Kelly, Acritical role for PPARalpha-mediated lipotoxicity in the pathogenesis of diabetic cardiomyopathy: modulation by dietary fat content, Proc Natl 
Acad Sci U S A, 100 (2003) 1226-1231.

43. N.H. Son, T.S. Park, H. Yamashita, M. Yokoyama, L.A. Huggins, K. Okajima, S. Homma, M.J. Szabolcs, L.S. Huang, I.J. Goldberg, Cardiomyocyte expression of PPARgamma leads to cardiac dysfunction in mice, J Clin Invest, 117 (2007) 2791-2801.

44. H.C. Chiu, A. Kovacs, R.M. Blanton, X. Han, M. Courtois, C.J. Weinheimer, K.A. Yamada, S. Brunet, H. Xu, J.M. Nerbonne, M.J. Welch, N.M. Fettig, T.L. Sharp, N. Sambandam, K.M. Olson, D.S. Ory, J.E. Schaffer, Transgenic expression of fatty acid transport protein 1 in the heart causes lipotoxic cardiomyopathy, Circ Res, 96 (2005) 225233.

45. J.M. Adams, 2nd, T. Pratipanawatr, R. Berria, E. Wang, R.A. DeFronzo, M.C. Sullards, L.J. Mandarino, Ceramide content is increased in skeletal muscle from obese insulin-resistant humans, Diabetes, 53 (2004) 25-31.

46. A. Chokshi, K. Drosatos, F.H. Cheema, R. Ji, T. Khawaja, S. Yu, T. Kato, R. Khan, H. Takayama, R. Knoll, H. Milting, C.S. Chung, U. Jorde, Y. Naka, D.M. Mancini, I.J. Goldberg, P.C. Schulze, Ventricular assist device implantation corrects myocardial lipotoxicity, reverses insulin resistance, and normalizes cardiac metabolism in patients with advanced heart failure, Circulation, 125 (2012) 2844-2853.

47. B.M. Spiegelman, J.S. Flier, Adipogenesis and obesity: rounding out the big picture, Cell, 87 (1996) 377-389.

48. S. Tyagi, P. Gupta, A.S. Saini, C. Kaushal, S. Sharma, The peroxisome proliferator-activated receptor: A family of nuclear receptors role in various diseases, J Adv Pharm Technol Res, 2 (2011) 236-240.

49. A.J. Gilde, K.A. van der Lee, P.H. Willemsen, G. Chinetti, F.R. van der Leij, G.J. van der Vusse, B. Staels, M. van Bilsen, Peroxisome proliferatoractivated receptor (PPAR) alpha and PPARbeta/ delta, but not PPARgamma, modulate the expression of genes involved in cardiac lipid metabolism, Circ Res, 92 (2003) 518-524.

50. A. Georgiadi, S. Kersten, Mechanisms of gene regulation by fatty acids, Adv Nutr, 3 (2012) 127134.

51. L. Cheng, G. Ding, Q. Qin, Y. Huang, W. Lewis, N. He, R.M. Evans, M.D. Schneider, F.A. Brako, Y. Xiao, Y.E. Chen, Q. Yang, Cardiomyocyte-restricted peroxisome proliferator-activated receptor-delta deletion perturbs myocardial fatty acid oxidation and leads to cardiomyopathy, Nat Med, 10 (2004) 1245-1250.

52. M. Wierzbicki, A. Chabowski, M. Zendzian-Piotrowska, J. Gorski, Differential effects of in vivo PPAR alpha and gamma activation on fatty acid transport proteins expression and lipid content in rat liver, J Physiol Pharmacol, 60 (2009) 99106.

53. B.N. Finck, J.J. Lehman, T.C. Leone, M.J. Welch, M.J. Bennett, A. Kovacs, X. Han, R.W. Gross, R. Kozak, G.D. Lopaschuk, D.P. Kelly, The cardiac phenotype induced by PPARalpha overexpression mimics that caused by diabetes mellitus, $J$ Clin Invest, 109 (2002) 121-130.

54. S.Y. Park, Y.R. Cho, B.N. Finck, H.J. Kim, T. Higashimori, E.G. Hong, M.K. Lee, C. Danton, S. Deshmukh, G.W. Cline, J.J. Wu, A.M. Bennett, B. Rothermel, A. Kalinowski, K.S. Russell, Y.B. Kim, D.P. Kelly, J.K. Kim, Cardiac-specific overexpression of peroxisome proliferator-activated receptor-alpha causes insulin resistance in heart and liver, Diabetes, 54 (2005) 2514-2524.

55. J. Yang, N. Sambandam, X. Han, R.W. Gross, M. Courtois, A. Kovacs, M. Febbraio, B.N. Finck, D.P. Kelly, CD36 deficiency rescues lipotoxic cardiomyopathy, Circ Res, 100 (2007) 1208-1217.

56. F. Djouadi, C.J. Weinheimer, J.E. Saffitz, C. Pitchford, J. Bastin, F.J. Gonzalez, D.P. Kelly, A gender-related defect in lipid metabolism and glucose homeostasis in peroxisome proliferator- activated receptor alpha- deficient mice, J Clin Invest, 102 (1998) 1083-1091.

57. J.M. Peters, N. Hennuyer, B. Staels, J.C. Fruchart, C. Fievet, F.J. Gonzalez, J. Auwerx, Alterations in lipoprotein metabolism in peroxisome proliferator-activated receptor alpha-deficient mice, J Biol Chem, 272 (1997) 27307-27312.

58. M. Guerre-Millo, C. Rouault, P. Poulain, J. Andre, V. Poitout, J.M. Peters, F.J. Gonzalez, J.C. Fruchart, G. Reach, B. Staels, PPAR-alpha-null mice are protected from high-fat diet-induced insulin resistance, Diabetes, 50 (2001) 2809. 2814.

59. T.I. Lee, Y.H. Kao, Y.C. Chen, J.H. Huang, F.C. Hsiao, Y.J. Chen, Peroxisome proliferator-activated receptors modulate cardiac dysfunction in diabetic cardiomyopathy, Diabetes Res Clin Pract, 100 (2013) 330-339.

60. E. Aasum, M. Cooper, D.L. Severson, T.S. Larsen, Effect of BM 17.0744, a PPARalpha ligand, on the metabolism of perfused hearts from control and diabetic mice, Can J Physiol Pharmacol, 83 (2005) 183-190.

61. E.M. Burkart, N. Sambandam, X. Han, R.W. Gross, M. Courtois, C.M. Gierasch, K. Shoghi, M.J. Welch, D.P. Kelly, Nuclear receptors PPARbeta/delta and PPARalpha direct distinct metabolic regulatory programs in the mouse heart, $J$ Clin Invest, 117 (2007) 3930-3939.

62. C.J. Pol, M. Lieu, K. Drosatos, PPARs: Protectors or Opponents of Myocardial Function?, PPAR Res, 2015 (2015) 835985. 
63. S.L. Coort, D.M. Hasselbaink, D.P. Koonen, J. Willems, W.A. Coumans, A. Chabowski, G.J. van der Vusse, A. Bonen, J.F. Glatz, J.J. Luiken, Enhanced sarcolemmal FAT/CD36 content and triacylglycerol storage in cardiac myocytes from obese zucker rats, Diabetes, 53 (2004) 1655-1663.

64. S. Boudina, H. Bugger, S. Sena, B.T. O’Neill, V.G. Zaha, O. Ilkun, J.J. Wright, P.K. Mazumder, E. Palfreyman, T.J. Tidwell, H. Theobald, O. Khalimonchuk, B. Wayment, X. Sheng, K.J. Rodnick, R. Centini, D. Chen, S.E. Litwin, B.E. Weimer, E.D. Abel, Contribution of impaired myocardial insulin signaling to mitochondrial dysfunction and oxidative stress in the heart, Circulation, 119 (2009) 1272-1283.

65. O. Ilkun, S. Boudina, Cardiac dysfunction and oxidative stress in the metabolic syndrome: an update on antioxidant therapies, Curr Pharm Des, 19 (2013) 4806-4817.

66. H. Bugger, E.D. Abel, Molecular mechanisms for myocardial mitochondrial dysfunction in the metabolic syndrome, Clin Sci (Lond), 114 (2008) 195-210.

67. M.A. Abdul-Ghani, R.A. DeFronzo, Pathogenesis of insulin resistance in skeletal muscle, $J$ Biomed Biotechnol, 2010 (2010) 476279.

68. B.G. Teodoro, F.G. Baraldi, I.H. Sampaio, L.H. Bomfim, A.L. Queiroz, M.A. Passos, E.M. Carneiro, L.C. Alberici, R. Gomis, F.G. Amaral, J. Cipolla-Neto, M.B. Araujo, T. Lima, S. Akira Uyemura, L.R. Silveira, E. Vieira, Melatonin prevents mitochondrial dysfunction and insulin resistance in rat skeletal muscle, J Pineal Res, 57 (2014) 155-167.

69. S. Serpillon, B.C. Floyd, R.S. Gupte, S. George, M. Kozicky, V. Neito, F. Recchia, W. Stanley, M.S. Wolin, S.A. Gupte, Superoxide production by $\mathrm{NAD}(\mathrm{P}) \mathrm{H}$ oxidase and mitochondria is increased in genetically obese and hyperglycemic rat heart and aorta before the development of cardiac dysfunction. The role of glucose-6-phosphate dehydrogenase-derived NADPH, Am J Physiol Heart Circ Physiol, 297 (2009) H153-162.

70. S.Y. Li, X. Yang, A.F. Ceylan-Isik, M. Du, N. Sreejayan, J. Ren, Cardiac contractile dysfunction in Lep/Lep obesity is accompanied by NADPH oxidase activation, oxidative modification of sarco(endo)plasmic reticulum Ca2+-ATPase and myosin heavy chain isozyme switch, Diabetologia, 49 (2006) 1434-1446.

71. N.D. Roe, D.P. Thomas, J. Ren, Inhibition of NADPH oxidase alleviates experimental diabetes-induced myocardial contractile dysfunction, Diabetes Obes Metab, 13 (2011) 465-473.

72. J.G. Duncan, Mitochondrial dysfunction in diabetic cardiomyopathy, Biochim Biophys Acta, 1813 (2011) 1351-1359.
73. J. Reid, A.I. Macdougall, M.M. Andrews, Aspirin and diabetes mellitus, $\mathrm{Br}$ Med J, 2 (1957) 10711074.

74. H.J. Ko, Z. Zhang, D.Y. Jung, J.Y. Jun, Z. Ma, K.E. Jones, S.Y. Chan, J.K. Kim, Nutrient stress activates inflammation and reduces glucose metabolism by suppressing AMP-activated protein kinase in the heart, Diabetes, 58 (2009) 2536-2546.

75. L. Cao, X. Qin, M.R. Peterson, S.E. Haller, K.A. Wilson, N. Hu, X. Lin, S. Nair, J. Ren, G. He, CARD9 knockout ameliorates myocardial dysfunction associated with high fat diet-induced obesity, J Mol Cell Cardiol, 92 (2016) 185-195.

76. I. Jialal, H. Kaur, S. Devaraj, Toll-like receptor status in obesity and metabolic syndrome: a translational perspective, J Clin Endocrinol Metab, 99 (2014) 39-48.

77. L.A. Velloso, F. Folli, M.J. Saad, TLR4 at the Crossroads of Nutrients, Gut Microbiota, and Metabolic Inflammation, Endocr Rev, 36 (2015) 245-271.

78. J.J. Kim, D.D. Sears, TLR4 and Insulin Resistance, Gastroenterol Res Pract, 2010 (2010).

79. C. Jin, R.A. Flavell, Innate sensors of pathogen and stress: linking inflammation to obesity, $\mathrm{J} \mathrm{Al}$ lergy Clin Immunol, 132 (2013) 287-294.

80. P. Plomgaard, K. Bouzakri, R. Krogh-Madsen, B. Mittendorfer, J.R. Zierath, B.K. Pedersen, Tumor necrosis factor-alpha induces skeletal muscle insulin resistance in healthy human subjects via inhibition of Akt substrate 160 phosphorylation, Diabetes, 54 (2005) 2939-2945.

81. J.K. Kim, Endothelial nuclear factor kappaB in obesity and aging: is endothelial nuclear factor kappaB a master regulator of inflammation and insulin resistance?, Circulation, 125 (2012) 10811083.

82. W.L. Holland, B.T. Bikman, L.P. Wang, G. Yuguang, K.M. Sargent, S. Bulchand, T.A. Knotts, G. Shui, D.J. Clegg, M.R. Wenk, M.J. Pagliassotti, P.E. Scherer, S.A. Summers, Lipid-induced insulin resistance mediated by the proinflammatory receptor TLR4 requires saturated fatty acid-induced ceramide biosynthesis in mice, J Clin Invest, 121 (2011) 1858-1870.

83. C.K. Glass, J.M. Olefsky, Inflammation and lipid signaling in the etiology of insulin resistance, Cell Metab, 15 (2012) 635-645.

84. M.T. Waddingham, A.J. Edgley, H. Tsuchimochi, D.J. Kelly, M. Shirai, J.T. Pearson, Contractile apparatus dysfunction early in the pathophysiology of diabetic cardiomyopathy, World J Diabetes, 6 (2015) 943-960.

85. K. Dutta, D.A. Podolin, M.B. Davidson, A.J. Davidoff, Cardiomyocyte dysfunction in sucrosefed rats is associated with insulin resistance, Diabetes, 50 (2001) 1186-1192. 
86. K.K. Hintz, J. Ren, Prediabetic insulin resistance is not permissive to the development of cardiac resistance to insulin-like growth factor I in ventricular myocytes, Diabetes Res Clin Pract, 55 (2002) 89-98.

87. K.K. Hintz, N.S. Aberle, J. Ren, Insulin resistance induces hyperleptinemia, cardiac contractile dysfunction but not cardiac leptin resistance in ventricular myocytes, Int J Obes Relat Metab Disord, 27 (2003) 1196-1203.

88. F.S. Fein, Diabetic cardiomyopathy, Diabetes Care, 13 (1990) 1169-1179.

89. J.P. Morgan, Abnormal intracellular modulation of calcium as a major cause of cardiac contractile dysfunction, N Engl J Med, 325 (1991) 625-632.

90. H. Hayashi, N. Noda, Cytosolic Ca2+ concentration decreases in diabetic rat myocytes, Cardiovasc Res, 34 (1997) 99-103.

91. L.E. Wold, K. Dutta, M.M. Mason, J. Ren, S.E. Cala, M.L. Schwanke, A.J. Davidoff, Impaired SERCA function contributes to cardiomyocyte dysfunction in insulin resistant rats, $J$ Mol Cell Cardiol, 39 (2005) 297-307.

92. A. Malhotra, S. Penpargkul, F.S. Fein, E.H. Sonnenblick, J. Scheuer, The effect of streptozotocininduced diabetes in rats on cardiac contractile proteins, Circ Res, 49 (1981) 1243-1250.

93. N. Takeda, I. Nakamura, T. Hatanaka, T. Ohkubo, M. Nagano, Myocardial mechanical and myosin isoenzyme alterations in streptozotocin-diabetic rats, Jpn Heart J, 29 (1988) 455-463.

94. V.L. Rundell, D.L. Geenen, P.M. Buttrick, P.P. de Tombe, Depressed cardiac tension cost in experimental diabetes is due to altered myosin heavy chain isoform expression, Am J Physiol Heart Circ Physiol, 287 (2004) H408-413.

95. D.J. Glenn, F. Wang, M. Nishimoto, M.C. Cruz, Y. Uchida, W.M. Holleran, Y. Zhang, Y. Yeghiazarians, D.G. Gardner, A murine model of isolated cardiac steatosis leads to cardiomyopathy, Hypertension, 57 (2011) 216-222.

96. L. Liu, X. Shi, K.G. Bharadwaj, S. Ikeda, H. Yamashita, H. Yagyu, J.E. Schaffer, Y.H. Yu, I.J. Goldberg, DGAT1 expression increases heart triglyceride content but ameliorates lipotoxicity, J Biol Chem, 284 (2009) 36312-36323.

97. G. Wolkart, A. Schrammel, K. Dorffel, G. Haemmerle, R. Zechner, B. Mayer, Cardiac dysfunction in adipose triglyceride lipase deficiency: treatment with a PPARalpha agonist, $\mathrm{Br}$ J Pharmacol, 165 (2012) 380-389.

98. A.J. Hoy, C.R. Bruce, S.M. Turpin, A.J. Morris, M.A. Febbraio, M.J. Watt, Adipose triglyceride lipase-null mice are resistant to high-fat diet-induced insulin resistance despite reduced energy expenditure and ectopic lipid accumulation, Endocrinology, 152 (2011) 48-58.
99. P.C. Kienesberger, T. Pulinilkunnil, J. Nagendran, M.E. Young, J.G. Bogner-Strauss, H. Hackl, R. Khadour, E. Heydari, G. Haemmerle, R. Zechner, E.E. Kershaw, J.R. Dyck, Early structural and metabolic cardiac remodelling in response to inducible adipose triglyceride lipase ablation, Cardiovasc Res, 99 (2013) 442-451.

100. T. Pulinilkunnil, P.C. Kienesberger, J. Nagendran, N. Sharma, M.E. Young, J.R. Dyck, Cardiac-specific adipose triglyceride lipase overexpression protects from cardiac steatosis and dilated cardiomyopathy following diet-induced obesity, Int J Obes (Lond), 38 (2014) 205-215.

101. C.J. Knowles, M. Cebova, I.M. Pinz, Palmitate diet-induced loss of cardiac caveolin-3: a novel mechanism for lipid-induced contractile dysfunction, PLoS One, 8 (2013) e61369.

102. R.G. Parton, K. Simons, The multiple faces of caveolae, Nat Rev Mol Cell Biol, 8 (2007) 185-194.

103. [103] R.G. Parton, M. Way, N. Zorzi, E. Stang, Caveolin-3 associates with developing T-tubules during muscle differentiation, J Cell Biol, 136 (1997) 137-154.

104. [104] F. Galbiati, J.A. Engelman, D. Volonte, X.L. Zhang, C. Minetti, M. Li, H. Hou, Jr., B. Kneitz, W. Edelmann, M.P. Lisanti, Caveolin-3 null mice show a loss of caveolae, changes in the microdomain distribution of the dystrophin -glycoprotein complex, and t-tubule abnormalities, J Biol Chem, 276 (2001) 21425-21433.

105. [105] S.E. Woodman, D.S. Park, A.W. Cohen, M.W. Cheung, M. Chandra, J. Shirani, B. Tang, L.A. Jelicks, R.N. Kitsis, G.J. Christ, S.M. Factor, H.B. Tanowitz, M.P. Lisanti, Caveolin-3 knockout mice develop a progressive cardiomyopathy and show hyperactivation of the p42/44 MAPK cascade, J Biol Chem, 277 (2002) 38988-38997.

106. [106] D.S. Park, S.E. Woodman, W. Schubert, A.W. Cohen, P.G. Frank, M. Chandra, J. Shirani, B. Razani, B. Tang, L.A. Jelicks, S.M. Factor, L.M Weiss, H.B. Tanowitz, M.P. Lisanti, Caveolin-1/3 double-knockout mice are viable, but lack both muscle and non-muscle caveolae, and develop a severe cardiomyopathic phenotype, $A m$ J Pathol, 160 (2002) 2207-2217.

107. [107] T. Hayashi, T. Arimura, K. Ueda, H. Shibata, S. Hohda, M. Takahashi, H. Hori, Y. Koga, N. Oka, T. Imaizumi, M. Yasunami, A. Kimura, Identification and functional analysis of a caveolin-3 mutation associated with familial hypertrophic cardiomyopathy, Biochem Biophys Res Commun, 313 (2004) 178-184.

108. [108] J. Oshikawa, K. Otsu, Y. Toya, T. Tsunematsu, R. Hankins, J. Kawabe, S. Minamisawa, S. Umemura, Y. Hagiwara, Y. Ishikawa, Insulin resistance in skeletal muscles of caveolin-3-null mice, Proc Natl Acad Sci U S A, 101 (2004) 12670-12675. 
109. [109] D.M. Muoio, T.R. Koves, Lipid-induced metabolic dysfunction in skeletal muscle, Novartis Found Symp, 286 (2007) 24-38; discussion 3846, 162-163, 196-203.

110. [110] D. Bonnet, D. Martin, L. Pascale De, E. Villain, P. Jouvet, D. Rabier, M. Brivet, J.M. Saudubray, Arrhythmias and conduction defects as presenting symptoms of fatty acid oxidation disorders in children, Circulation, 100 (1999) 22482253.

111. [111] G. Schmilinsky-Fluri, V. Valiunas, M. Willi, R. Weingart, Modulation of cardiac gap junctions: the mode of action of arachidonic acid, $J$ Mol Cell Cardiol, 29 (1997) 1703-1713.

112. [112] R.H. Unger, L. Orci, Diseases of liporegulation: new perspective on obesity and related disorders, FASEB J, 15 (2001) 312-321.

113. [113] K. Drosatos, P.C. Schulze, Cardiac lipotoxicity: molecular pathways and therapeutic implications, Curr Heart Fail Rep, 10 (2013) 109-121.
114. T.S. Park, I.J. Goldberg, Sphingolipids, lipotoxic cardiomyopathy, and cardiac failure, Heart Fail Clin, 8 (2012) 633-641.

115. J.S. Lally, L.A. Snook, X.X. Han, A. Chabowski, A. Bonen, G.P. Holloway, Subcellular lipid droplet distribution in red and white muscles in the obese Zucker rat, Diabetologia, 55 (2012) 479488.

116. M.J. Morgan, Z.G. Liu, Crosstalk of reactive oxygen species and NF-kappaB signaling, Cell Res, 21 (2011) 103-115.

117. R. Harmancey, H. Taegtmeyer, The complexities of diabetic cardiomyopathy: lessons from patients and animal models, Curr Diab Rep, 8 (2008) 243-248. 



\title{
Chapter 3
}

\section{Central role of dysassembly of vacuolar-type $\mathrm{H}^{+}$-ATPase in lipid-induced cardiomyopathy}

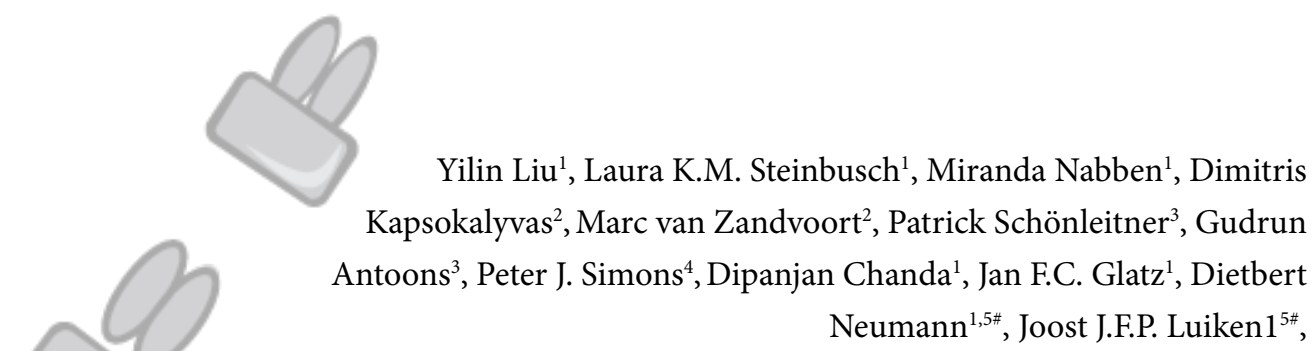

Departments of ${ }^{1}$ Molecular Genetics, ${ }^{2}$ Molecular Cell Biology and ${ }^{3}$ Physiology, CARIM School for Cardiovascular Diseases, Maastricht University, P.O. Box 616, 6200 MD Maastricht, the Netherlands ${ }^{4}$ EPIRUS Biopharmaceuticals Netherlands BV, 3584 CM Utrecht, the Netherlands ${ }^{5} \mathrm{Co}$-senior author

"Correspondence: d.neumann@maastrichtuniversity.nl and j.luiken@maastrichtuniversity.nl 


\begin{abstract}
Dietary fat overconsumption leads to myocardial lipid accumulation through unknown mechanisms. Previously, we identified increased translocation of the fatty acid transporter CD36 from its endosomal storage compartment to the sarcolemma as primary mechanism of excessive myocellular lipid import. Here, we show that increased CD36 translocation is caused by alkalinization of endosomes due to inhibition of proton pumping activity of vacuolar-type $\mathrm{H}^{+}$-ATPase (v-ATPase). Endosomal alkalinization was observed in hearts from rats fed a high fat-diet and in rodent and human cardiomyocytes upon lipid overexposure, and appeared as early lipid-induced event preceding the onset of insulin resistance. Inhibition of v-ATPase reduced insulin sensitivity and cardiomyocyte contractility, which was rescued by CD36 silencing. The mechanism of lipid-induced v-ATPase inhibition involves migration of the ATPase-containing sub-complex into the cytoplasm after its disassembly from the membrane-bound proton channel sub-complex. Hence, lipid oversupply increases CD36-mediated myocellular lipid accumulation, which is sensed by v-ATPase and results in its disassembly. The consequent endosomal alkalinization further increases CD36 translocation and, lipid uptake. This feed-forward mechanism of lipid-stimulated lipid uptake progressively produces myocardial lipid accumulation, which ultimately impairs cardiac insulin sensitivity and contractility. In conclusion, lipid-induced v-ATPase inhibition is a physiological signal that, if persistent, precipitates into lipid-induced cardiomyopathy.
\end{abstract}




\section{Introduction}

Overconsumption of lipid-containing products is associated with a high risk of developing heart failure. A causal link between myocardial triacylglycerol (TAG) accumulation and decreased contractile function is found in both diabetic patients and insulin resistant rat models $[1,2]$. In the insulin-resistant condition, cardiac dysfunction is increasingly being related to intramyocardial accumulation of lipid metabolites, such as diacylglycerols and ceramides. Myocardial lipid accumulation is the result of a sustained high rate of long-chain fatty acid (LCFA) uptake that exceeds its rate of oxidation [3]. The mechanisms of chronically elevated LCFA uptake involve altered dynamics of LCFA transporters [3].

Whereas the heart expresses four membrane LCFA transporters (CD36, FABPpm, FATP1, FATP4) [3], our studies with CD36 null animals have indicated that $~ 70 \%$ of cardiac LCFA uptake is mediated via CD36. In the heart, CD36 is not only present at the plasma membrane, but also stored in intracellular membrane compartments (endosomes), from where it can translocate to the sarcolemma to increase LCFA uptake [3]. Insulin is the main hormonal stimulus to induce CD36 translocation leading to increased LCFA uptake for subsequent storage into triacylglycerols [3, 4]. Insulin-induced CD36 translocation closely resembles the well-characterized insulin-induced GLUT4 translocation from endosomes to the cell surface to increase cardiac glucose uptake [3]. Hence, CD36 translocation is a key regulatory mechanism of cardiac LCFA uptake.

Studies in rodent models of insulin resistance have shown a chronically elevated uptake of LCFA into cardiomyocytes that is coupled to enhanced esterification into TAG and formation of diacylglycerols/ceramides [5,6]. This chronically elevated LCFA influx is not due to changes in CD36 protein expression, but rather to increased translocation of CD36 from endosomes to the sarcolemma, indicating that lipid oversupply induces chronic changes in subcellular CD36 cycling. Diacylglycerols and ceramides have been shown to interfere with insulin signaling at the level of IRS1/2 or Akt2 [7], which then leads to inhibition of insulin-stimulated GLUT4 translocation and glucose uptake [3, 6]. Insulin resistance therefore would be expected to also impair insulin-stimulated CD36 translocation and LCFA uptake. However, as mentioned above, CD36-dependent lipid uptake is highly upregulated in the insulin resistant heart. Hence, it remains elusive how increased CD36 translocation evolves from lipid oversupply.

Vacuolar-type $\mathrm{H}^{+}$-ATPase (v-ATPase) is a key protein involved in vesicular trafficking [8]. By active import of protons v-ATPase acidifies the lumen of vesicular organelles, among which endosomes. The multimeric v-ATPase protein complex consists of 14 subunits: 6 subunits form the integral membrane sub-complex $V_{o}$, encompassing the proton channel; 8 subunits make up a peripheral membrane sub-complex $\mathrm{V}_{1}$, containing 
the ATP-binding pocket $[9,10]$. Previously, we obtained preliminary evidence that endosomal alkalinization causes a rapid expulsion of CD36, not of GLUT4, to the sarcolemma, suggesting that proper functioning of v-ATPase is required for CD36 retention in the endosomes [11]. Taking this a step further lead us to hypothesize that increased CD36 translocation in the lipid-overloaded heart is due to an inhibition of v-ATPase. This scenario would provide v-ATPase, as a putative lipid sensor, with a novel role in the pathology of lipid-induced cardiomyopathy.

In this study, we first analyzed v-ATPase function in cardiomyocytes in vivo and in vitro during chronic lipid oversupply. Secondly, we made a detailed assessment in time of the onset of v-ATPase inhibition compared to increased LCFA uptake and insulin resistance. Thirdly, we studied if genetic or pharmacological inhibition of v-ATPase affects insulin resistance and contractile dysfunction, and clarified the role of CD36. Fourthly, we investigated functional alterations of $\mathrm{v}$-ATPase in human stem cell-derived cardiomyocytes subjected to excess lipids. Finally, we determined the mechanism of v-ATPase inhibition

\section{Methods}

\section{Experimental animals, isolation and culturing of primary rat cardiomyocytes.}

Male Lewis rats, 200-250 grams, were purchased from Charles River laboratories and fed either with 10\% low-fat diet (Research Diets D12450B, New Brunswick, NJ) or 60\% high-fat diet (D12492). After 7 weeks, cardiomyocytes were isolated as previously described [12]. Culturing was performed for up to $48 \mathrm{~h}$ in low-palmitate and highpalmitate media as previously described [13]. For selected experiments, cultured primary cardiomyocytes were treated with a blocking antibody against CD36 (Clone 63, provided by EPIRUS Biopharmaceuticals Netherlands BV) as previously described [13].

\section{Cell culture of HL-1 cardiomyocytes and transfection.}

HL-1 cells were cultured in Control medium as previously described [14]. We also used low-palmitate and high-palmitate media containing $500 \mathrm{nM}$ palmitate and $20 \mu \mathrm{M}$ palmitate, respectively, complexed to $3.3 \mu \mathrm{M}$ bovine serum albumin (BSA), resulting in palmitate/BSA ratios of 0.15:1 (low-palmitate) and 6:1 (high-palmitate) in the presence of $100 \mathrm{nM}$ insulin. Cells were transfected at 60-70\% confluence, $24 \mathrm{~h}$ after seeding. Transfection of siRNA was done with 10 pmol of siRNA and $2 \mu \mathrm{l}$ of Lipofetamine RNAiMAX per well in antibiotic- and noradrenaline-free culture medium. After $6 \mathrm{~h}$, medium was refreshed with growth medium, and $48 \mathrm{~h}$ after transfection cell lysis or functional assays were performed.

Human iPSCs differentiated into cardiomyocytes.

Human iPSCs were kindly provided by F. van Tienen (Maastricht University Medical Center), and differentiated into cardiomyocytes according to manufacturers' protocol 
(Thermo Fisher Scientific). All treatments on iPSC-CM were performed as indicated for HL-1 cardiomyocytes.

CD36 cell surface staining.

Colorimetric detection of CD36 at the sarcolemma using an HRP-linked secondary antibody was carried out as previously described [15]. Two-photon microscopy to visualize cell surface localization of CD36 was performed as previously described [13].

Measurement of substrate uptake.

$\left[1-{ }^{14} \mathrm{C}\right]$ palmitate and $\left[1-{ }^{3} \mathrm{H}\right]$ deoxyglucose uptake into suspensions of primary cardiomyocytes was measured as previously described [4]. Uptake of these substrates into HL-1 cardiomyocytes and into cultured primary cardiomyocytes, both cell models seeded on pre-coated glass slides, was measured as previously described [13, 14].

Measurement of triacylglycerol content.

Myocellular accumulation of triacylglycerol was measured as previously described [15].

Measurement of cellular chloroquine accumulation as readout of V-ATPase function.

Accumulation of Chloroquine into freshly isolated cardiomyocytes was measured as previously described [11]. Chloroquine accumulation into cultured cells was essentially done in a similar manner with small modifications. Briefly, upon 20 min treatment with/ without $100 \mathrm{mM}$ Bafilomycin-A, $2 \mu \mathrm{l}\left[{ }^{3} \mathrm{H}\right]$ chloroquine $(250 \mu \mathrm{Ci} / \mathrm{ml}$ diluted 1:125 in Milli-Q) was added per well containing 50,000 primary cardiomyocytes or 100,000 HL-1 cardiomyocytes. After a 20-min incubation period, the supernatant was aspirated. Cardiomyocytes were quickly washed with PBS once, and lysed with $1 \mathrm{M} \mathrm{NaOH}$ for 20 min at room temperature, and used for scintillation counting. The lysates were then transferred to $5 \mathrm{ml}$ Opti-Fluor (Perkin Elmer, Waltham, MA) until the pellet was completely dissolved. Samples were used for scintillation counting.

Microscopical imaging of endosomal acidification.

Primary cardiomyocytes were cultured for $1.5 \mathrm{~h}$ in adhesion medium, and then incubated for $15 \mathrm{~min}$ with $500 \mathrm{nM}$ lysosensor DND-189 and subsequent imaging with Leica TCS SP5 (Leica Microsystems GmbH, Wetzlar, Germany) two-photon microscope. Then, $100 \mathrm{nM}$ Bafilomycin-A (BafA) was added and a second image was captured. The excitation was at $820 \mathrm{~nm}$ and a Leica objective HCX APO L 20x/1.00 was used for excitation and epi-collection. Lysosensor green (DND-189, Invitrogen) fluorescence signal was detected at $470-550 \mathrm{~nm}$. We used a two-photon microscope instead of a confocal, because we intended to use the fluorescence lifetime method as a $\mathrm{pH}$ probe. 
Unfortunately lysosensor staining did not depend monotonically with $\mathrm{pH}$, so that calibration was not possible. Therefore only intensity images are presented.

Measurement of cardiomyocytic contraction dynamics.

Contractile properties of cardiomyocytes were assessed at $1 \mathrm{~Hz}$ field stimulation using a video-based cell geometry system to measure sarcomere dynamics (IonOptix). From the digitized recordings acquired with IonWizard acquisition software, the following parameters were calculated: sarcomere shortening, time to peak, and decay time. This was done as previously described [13].

\section{Measurement of co-localization of V-ATPase and CD36.}

Cardiomyocytes suspensions were subjected to subcellular fractionation as previously described [4]. This procedure yields the low-density microsomal fraction, which is enriched in endosomes. An aliquot of this fraction $(100 \mu \mathrm{g})$ was incubated with either anti-IgG or anti-CD36 or anti-vATPase a 2 antibodies overnight at $4{ }^{\circ} \mathrm{C}$ in the absence of detergents. Then, the antibody-captured low-density microsomal vesicles were coupled to protein A Sepharose 4 beads for $4 \mathrm{~h}$ at $4{ }^{\circ} \mathrm{C}$. Further preparation of the vesicles for analysis of co-immunoprecipitation was performed as earlier described [14].

\section{Measurement of V-ATPase disassembly.}

Two methods were applied for the disassembly measurement. (i) Immunoprecipitation: HL-1 cardiomyocytes were washed with ice cold PBS twice and lysed with lysis buffer containing 1\% Brij O20, $250 \mathrm{mM} \mathrm{NaCl}, 5 \mathrm{mM}$ EDTA and $50 \mathrm{mM}$ HEPES (pH 7.0). An aliquot of $500 \mu \mathrm{g}$ cell suspension was incubated with control IgG, or specific antibodies recognizing v-ATPase B2 or a2 subunits, overnight at $4{ }^{\circ} \mathrm{C}$. The next day, the proteinantibody complex was coupled to Sepharose 4 beads for $4 \mathrm{~h}$ at $4{ }^{\circ} \mathrm{C}$. Beads were washed $5 \mathrm{x}$ in lysis buffer, boiled in sample buffer, spun down, after which the supernatant was used for Western blotting. (ii) Fractionation: HL-1 cardiomyocytes were washed with ice cold PBS and scraped in ice cold SET buffer (10 mM Tris, $2 \mathrm{mM}$ EDTA and $250 \mathrm{mM}$ sucrose). The cell suspensions were frozen in liquid $\mathrm{N}_{2}$ and thawed for 3 cycles. Immediately thereafter the cell suspensions were centrifuged for $60 \mathrm{~min}$ at 200,000 g. Subsequently, the pellet, containing subcellular membranes, was resuspended in $200 \mu \mathrm{l}$ SET buffer. Additionally, the supernatant, representing cytoplasm, was collected. Both pellet and supernatant fractions were analyzed by Western blotting.

\section{Cell lysis and Western blotting.}

Cell lysis and Western blotting were performed as described earlier [4]. The signals were normalized to caveolin-3 (loading control). Then, a second normalization was carried out by setting the control incubation (low-fat diet, Ctrl or low-palmitate condition, 
depending on the experiment, see Figure legends details) at 1.

$m R N A$ expression of cardiac-specific genes.

Total RNA was isolated as previously described [16], and RT-qPCR was performed using SybrGreen (Bio-Rad) and the following primer sequences $\left(5^{\prime} \rightarrow 3^{\prime}\right)$ :

CTCCGTGAAGGGATAACCAGG (MYH6-F), GGGCCTCTAGACGCTCCTT (MYH6-R), AGCGGAAAAGTGGGAAGAGG (TNNT2-F), CACAGCTCCTTGGCCTTCTC (TNNT2-R), CACGAACCACGGCACTGATT (TBP-F), and TTTTCTTGCTGCCAGTCTGGAC (TBP-R). Samples were normalized using TBP as a housekeeping gene.

\section{Statistics.}

All data are presented as means \pm SEM. Statistical analysis was performed by using two-sided Student's t-test, and when possible we applied paired testing. P-values of less than 0.05 were considered statistically significant.

\section{Results}

In the rodent heart, CD36 co-localizes with $v$-ATPase on endosomes.

To determine whether CD36 co-localizes on endosomes with v-ATPase, rat cardiomyocytes were fractionated for the purification of the endosome-containing lowdensity microsomal fraction. As expected, the v-ATPase subunits a 2 and B2 were both more prominently present in the microsomal fraction compared to the total cardiomyocyte lysate (Figure 1A). This fraction was subsequently used for immunoprecipitation in the absence of detergents (i.e., to keep the vesicular membranes intact). Upon immunoprecipitation using CD36-directed antibodies, all of the a 2 subunit and most of the $\mathrm{B} 2$ subunit was found in the pellet-fraction, whereas no a2 and few B2 were found in the supernatant fraction (Figure 1A). In a reverse immunoprecipitation, using antibodies against a2, not all of the a2 was pelleted, presumably therefore also not all of B2. Yet, CD36 was almost completely captured by a2 antibodies (Figure 1A). Hence, under basal conditions the majority of CD36 and v-ATPase collocate to the same endosomal vesicles.

Lipotoxic conditions decrease V-ATPase function in cardiomyocytes in vivo and in vitro.

We first assessed v-ATPase function in a rodent model of lipid overconsumption and associated lipid-induced cardiomyopathy. Lewis rats were subjected for 7 weeks to a low fat diet ( $10 \%$ fat) or a high fat diet (60\% fat). As expected, body weight and heart mass were significantly increased in the high-fat diet group (Supplemental Figure 1, A and B), whereas blood glucose levels remained in the normal range (Supplemental Figure 1C). Upon subcellular fractionation, cardiomyocytes from high-fat diet fed rats displayed 
(A)

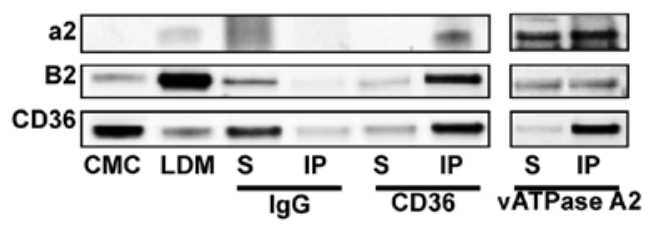

(B)

(D)
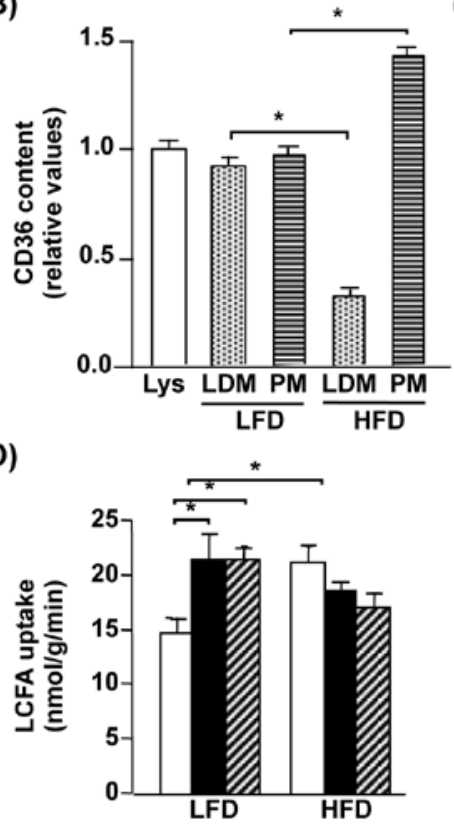

(F)

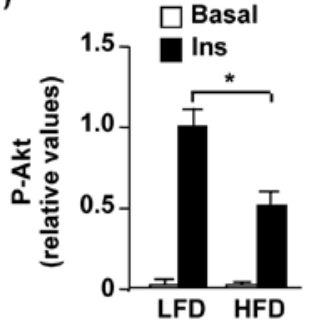

(I)

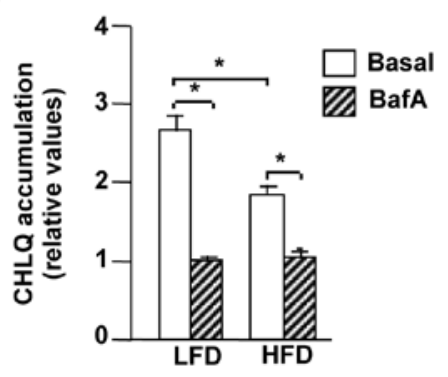

(G)
(C)

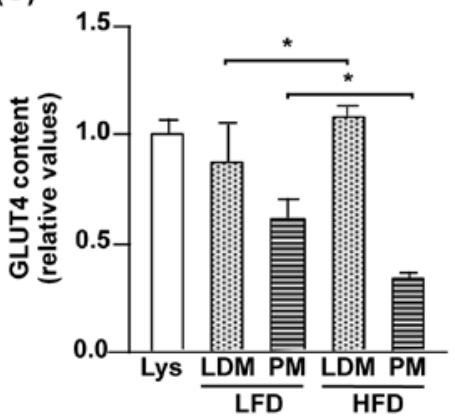

(E)

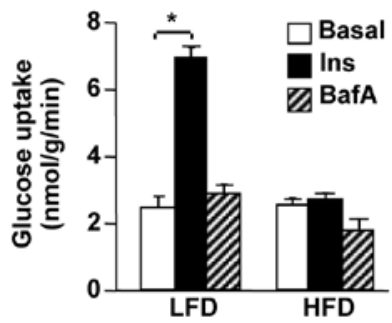

(H)
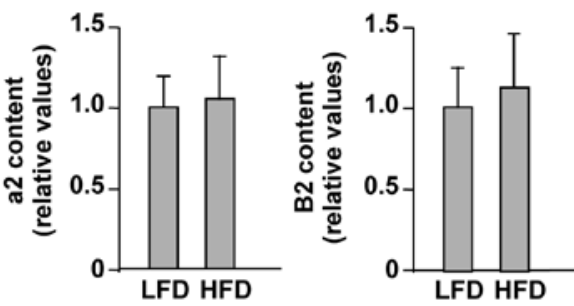

(J)

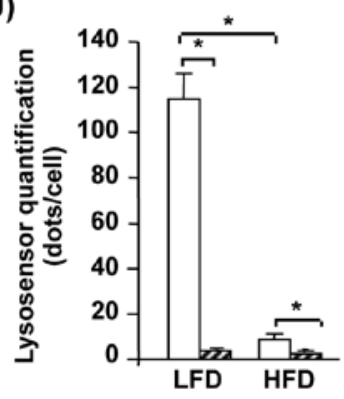


Figure 1. High-Fat Diet Impairs V-ATPase Function in Cardiomyocytes. (A) Immunoprecipitation (IP) of CD36 or v-ATPase subunit a2 or IgG control from LDM fractions prepared from primary rat cardiomyocytes. IP samples were immunoblotted with antibodies against CD36 and v-ATPase subunits $\mathrm{a} 2$ and B2. Immunoblots are representative results $(\mathrm{n}=3)$. (B,J) Cardiomyocytes were isolated from Lewis rats after 7 weeks on either low fat diet (LFD) or high fat diet (HFD). (B,C) CD36 and GLUT4 presence in cell lysate (Lys), at the plasma membrane fraction (PM) and in the low-density microsomal fraction (LDM) after subcellular fractionation $(n=3)$. (D,E) LCFA and glucose uptake into cardiomyocytes treated without/with insulin (Ins) or Bafilomycin-A (BafA) for 15 min (LFD: n=6; HFD: $\mathrm{n}=10$ ). (F) Akt phosphorylation (P-Akt) after treatment of cardiomyocyte suspensions without/ with 100 nM Ins (LFD: $n=4$; HFD: $n=4)$. (G,H) Protein expression of v-ATPase subunit a2 or B2 in cardiomyocytes (LFD: $\mathrm{n}=4$; HFD: $\mathrm{n}=4$ ). (I) Chloroquine (CHLQ) accumulation in cardiomyocytes treated without/with BafA for 15 min (LFD: $n=5$; HFD: $n=6$ ). (J) Quantification of lysosensor imaging in cardiomyocytes treated without/with BafA for $15 \mathrm{~min}$. Quantification is expressed as number of dots per cell (LFD: $n=6$; HFD: $n=6$; from each cardiomyocyte preparation, 5 cells were analyzed). Values are displayed as mean \pm SEM. ${ }^{*} \mathrm{p}<0.05$; N.S., not significant. Data were normalized to Lys $(\mathbf{B}, \mathbf{C})$, LFD/Basal (F) LFD (G,H) or to LFD/BafA $(\mathbf{I}, \mathbf{J})$.

To investigate a possible causal link between v-ATPase inhibition and increased CD36-mediated LCFA uptake, cardiomyocytes from low- and high-fat diet rats were incubated with Bafilomycin-A for shortterm (20 min). Bafilomycin-A stimulated LCFA uptake to a similar level as insulin in low-fat diet cardiomyocytes, whereas no significant effect of the inhibitor was observed in high-fat diet cardiomyocytes (Figure 1C). These data suggest that the mechanism of increased basal LCFA uptake that is seen in high-fat diet cardiomyocytes includes v-ATPase inhibition. Notably, the short-term treatment with Bafilomycin-A did not alter glucose uptake (Figure 1C). Therefore, CD36 trafficking is regulated by v-ATPase function, whereas GLUT4 is not.

increased localization of CD36 at the plasma membrane and decreased content in the lowdensity microsomal fraction (that includes endosomes). In contrast, GLUT4 localization showed the opposite response: increased low-density microsomal and decreased plasma membrane abundance (Supplemental Figure 1D). Hence in the high-fat diet condition, CD36 preferentially translocated to the sarcolemma whereas GLUT4 internalized to the microsomal fraction (Figure 1, B and C). Increased CD36 translocation to the sarcolemma was associated with increased basal LCFA uptake (Figure 1D). Furthermore, short-term (20 $\mathrm{min}$ ) insulin treatment stimulated LCFA and glucose uptake into cardiomyocytes in the low-fat diet, but not the high-fat diet, condition (Figure 1, D and E). Also insulininduced Akt phosphorylation was disturbed in the high-fat diet condition (Supplemental Figure 1E; quantification in Figure 1F). These findings are in line with previous observations in rats fed a similar high-fat diet [6].

Focusing on v-ATPase, the protein expression levels of the $\mathrm{V}_{0}$ subunit a2 and $\mathrm{V}_{1}$ subunit B2 were unaffected by the diet (Figure 1, G and H). As readout of v-ATPase function, we measured cellular accumulation of the divalent weak base Chloroquine, which becomes specifically trapped in acidic organelles [17]. Incubation of low-fat diet cardiomyocytes for 20 min with Bafilomycin-A (a potent v-ATPase inhibitor) decreased the amount of cell-associated Chloroquine by $62 \%$ (Figure 1I). Therefore, the Bafilomycin-A sensitive component of the cellular Chloroquine accumulation reflects the pumping function of v-ATPase-containing (acidic) organelles, such as endosomes. The 
(A)

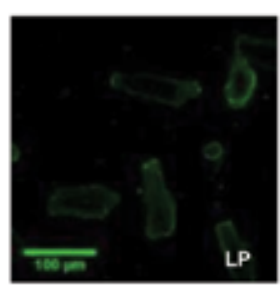

(C)

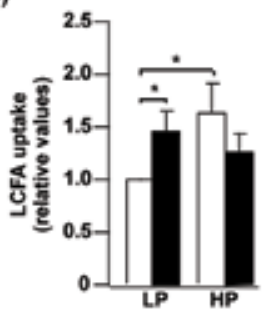

(F)

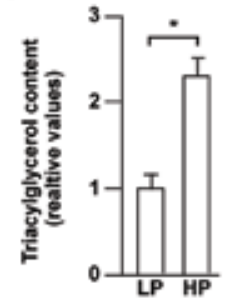

(D)

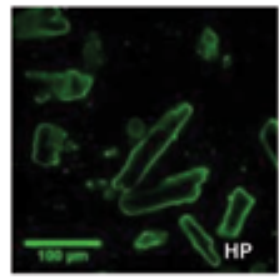

(E)
(B)

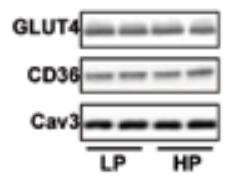

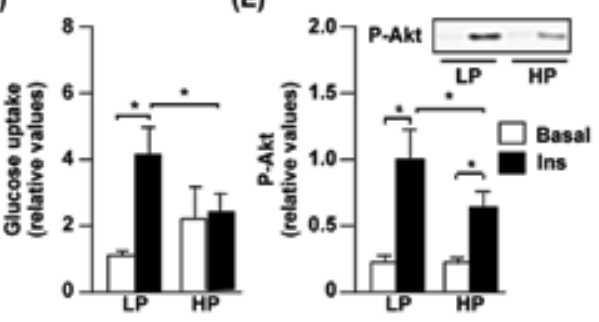

(G)

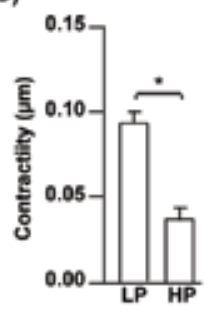

(H)

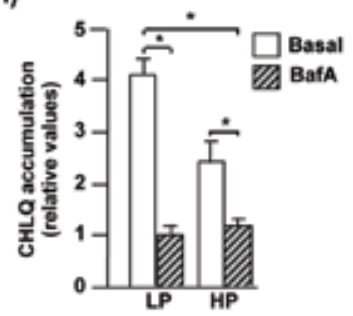

Figure 2. Lipid Overload Induces V-ATPase Inhibition in Cultured Cardiomyocytes. Rat cardiomyocytes were cultured for $48 \mathrm{~h}$ in low palmitate (LP) and high palmitate (HP)-containing media. (A) Cell surface abundance of CD36 protein using two-photon microscopy $(\mathrm{n}=3$; representative images are shown). (B) Protein expression of GLUT4 and CD36. Caveolin-3 (Cav3): loading control $(n=3)$. (C-E) LCFA and glucose uptake $(n=5)$ and Akt phosphorylation $(n=5)$ in cardiomyocytes treated without/with $100 \mathrm{nM}$ insulin (Ins) for $15 \mathrm{~min}$. Representative blot is shown. (F) Triacylglycerol content $(\mathrm{n}=5)$. (G) Sarcomeric shortening at $1 \mathrm{~Hz}$ field stimulation ( $\mathrm{n}=5$; imaging of 3 cells/condition). (H) Chloroquine (CHLQ) accumulation in cardiomyocytes treated without/with $100 \mathrm{nM}$ BafA for 15 $\min (n=5)$. Values are displayed as mean \pm SEM. ${ }^{*}<<0.05$; N.S., not significant. Data were normalized to LP/Basal (C,D), LP/Ins (E), LP (F) or to LP/BafA (H).

Bafilomycin-A insensitive cellular Chloroquine accumulation is likely due to cytoplasmic Chloroquine accumulation, because of the $\mathrm{pH}$ difference between cytoplasm ( $\mathrm{pH} 7.0$ ) and extracellular medium ( $\mathrm{pH}$ 7.4). Whereas the Bafilomycin-A insensitive component was unaltered in high-fat diet cardiomyocytes, the Bafilomycin-A sensitive component, i.e., v-ATPase function, was decreased by $52 \%$ (Figure 1I). To assess v-ATPase function in high-fat diet cardiomyocytes with an independent method, we used the fluorescent $\mathrm{pH}$ indicator Lysosensor DND-189 in combination with two-photon microscopy. Application of Lysosensor to low-fat diet cardiomyocytes yielded a particulate staining pattern (gray dots, Supplemental Figure 1F). Subsequent incubation with Bafilomycin-A resulted in 
a near-complete disappearance of the dots within $15 \mathrm{~min}$, suggesting that Lysosensor specifically visualizes organelles that are acidified by v-ATPase (Figure 1J and Supplemental Figure $1 \mathrm{~F}$ ). Importantly, the number of dots per cell in comparison to the low-fat condition was much lower in high-fat diet cardiomyocytes (Figure 1J and Supplemental Figure 1F). Taken together, both biochemical and microscopic methods indicate that v-ATPase function is inhibited in the lipid-overloaded heart.

Further we investigated the changes in v-ATPase activity in cardiomyocytes upon overexposure to lipids in vitro. Therefore, primary rat cardiomyocytes were cultured for 48 $\mathrm{h}$ in media containing either a low palmitate or high palmitate concentration. The highpalmitate culturing condition induced an elevation in surface CD36 content without changing CD36 total expression, indicating increased CD36 translocation to the sarcolemma (Figure 2, A and B). Accordingly, LCFA uptake (Figure 2C) and triacylglycerol content (Figure 2F) were increased upon high-palmitate treatment while insulinstimulated LCFA uptake, glucose uptake and Akt phosphorylation were decreased, and contractile function was impaired (Figure 2, C-E and G). Moreover, we observed a 60\% decrease in v-ATPase function in cardiomyocytes treated with high-palmitate versus lowpalmitate (Bafilomycin-A sensitive Chloroquine accumulation; Figure $2 \mathrm{H}$. Similar results were seen in mouse-derived HL-1 cardiomyocytes upon lipid oversupply (Supplemental Figure 2). Hence, cellular lipid oversupply decreases v-ATPase function and leads to insulin resistance and contractile dysfunction.

\section{Lipid oversupply causes V-ATPase inhibition before the onset of insulin resistance.}

To explore the onset of lipid-induced inhibition of v-ATPase relative to that of lipidinduced insulin resistance, primary cardiomyocytes were exposed to variable periods of high-palmitate culturing for up to $25 \mathrm{~h}$. We assessed v-ATPase function (by Chloroquine accumulation), as well as LCFA/glucose uptake and Akt/S6 phosphorylation in the absence or presence of insulin. From the Chloroquine data (Figure 3A) the Bafilomycin-A sensitive component was deduced (Supplemental Figure 3A), because it better reflects v-ATPase activity as discussed in the previous subsection. Similarly, for substrate uptake and phosphorylation status of Akt/S6, the insulin-sensitive component was calculated (Supplemental Figure 3, B-E). Under low-palmitate culturing conditions, endosomal $\mathrm{pH}$, substrate uptake and insulin sensitivity did not change (Figure 3, A-E and Supplemental Figure 3, A-E). In contrast, HP culturing induced a rapid 34\% decrease in $\mathrm{v}$-ATPase function already within the first hour, which was followed by a slower rate of decline for up to $25 \mathrm{~h}$ (Figure 3A). Additionally, already within the first hour there was a rapid 1.5-fold increase in basal LCFA uptake with no further increase thereafter (Figure 3B). This rapid increase in basal LCFA uptake was accompanied by a rapid loss of insulinsensitive LCFA uptake (Figure 3B and Supplemental Figure 3B). Reductions in insulin- 
(A)

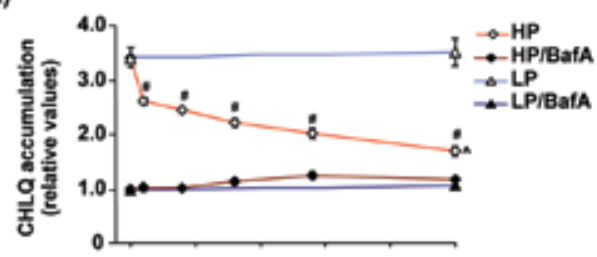

(C)

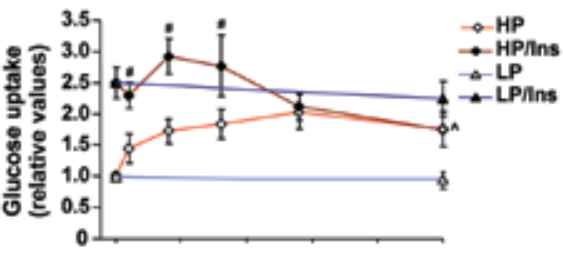

(E)

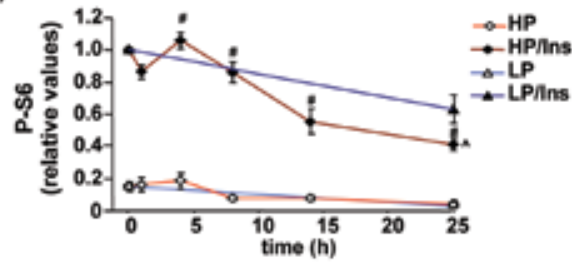

(B)

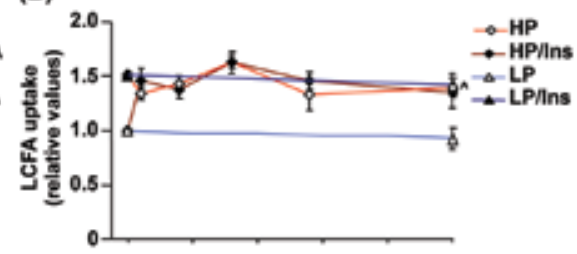

(D)

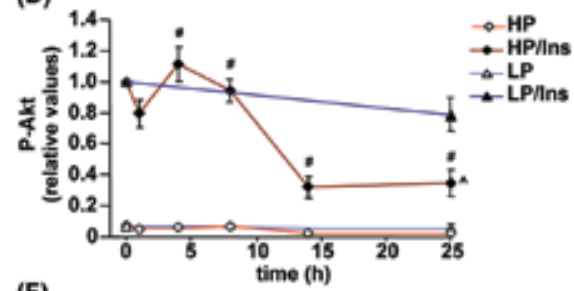

(F)

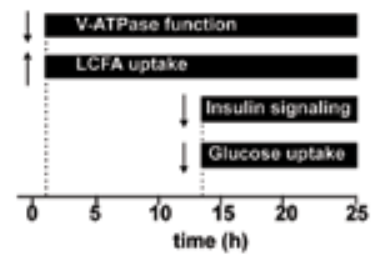

Figure 3. V-ATPase Inhibition Is an Early Event in Lipid-induced Insulin Resistance. Rat cardiomyocytes were cultured with either low palmitate (LP) or high palmitate (HP) for $0,1,3,8,15$, and maximum to $25 \mathrm{~h}$. (A) Chloroquine (CHLQ) accumulation in cardiomyocytes treated without/ with $100 \mathrm{nM}$ Bafilomycin-A (BafA) for $15 \mathrm{~min}(\mathrm{n}=6)$. (B-E) LCFA $(\mathrm{n}=4-6)$ and glucose $(\mathrm{n}=6)$ uptake and phosphorylation of Akt (P-Akt; $n=6)$ and S6 (P-S6; $n=6)$ in cardiomyocytes treated without/with $100 \mathrm{nM}$ insulin (Ins) for $15 \mathrm{~min}$. Values are displayed as mean \pm SEM. ${ }^{*} \mathrm{p}<0.05$; N.S., not significant. Data was normalized to $\mathrm{LP} / \mathrm{t}=0(\mathbf{A}-\mathbf{C})$ or to $\mathrm{LP} / \mathrm{Ins} / \mathrm{t}=0(\mathbf{D}, \mathbf{E})$. (F) Overview of the data shown in $(\mathbf{A}$ E), in order to highlight endosomal function and lipid accumulation as early events before onset of insulin resistance.

sensitive glucose uptake (Figure 3C and Supplemental Figure 3C) and phosphorylation of Akt (Figure 3D and Supplemental Figure 3, D and E) and S6 (Figure 3E, Supplemental Figure 3, D and F) occurred much later, namely after $14 \mathrm{~h}$ of high-palmitate culturing. In conclusion, high-palmitate induces a set of early changes starting within $1 \mathrm{~h}$ and a set of later changes occurring after $14 \mathrm{~h}$ (Figure 3F). Hence, v-ATPase inhibition arises well before the emergence of insulin resistance, which is suggestive of v-ATPase inhibition as a mediator of lipid-induced insulin resistance.

Direct inhibition of V-ATPase induces insulin resistance.

We explored v-ATPase inhibition as crucial mechanism in lipid-induced insulin resistance. Inhibition of v-ATPase in HL-1 cardiomyocytes was achieved genetically via silencing of subunit B2 (which also led to downregulation of subunit a2), and pharmacologically via long-term Bafilomycin-A treatment (Supplemental Figure 4A, 
quantified in Figure 4, A and B). Both treatments decreased v-ATPase function by $>70 \%$ (Bafilomycin-A sensitive Chloroquine accumulation; Figure 4C and Supplemental Figure 4B). Subsequently, the effect of v-ATPase inhibition on insulin sensitivity was investigated in culturing media with low palmitate concentrations that by themselves did not induce CD36 translocation or insulin resistance (Supplemental Figure 4, C-F and Figure 4, D-F: compare Ctrl and LP). Similarly to high-palmitate culturing conditions, both genetic and pharmacological v-ATPase inhibition caused a $>1.6$-fold increase in sarcolemmal CD36 content under basal conditions, which occurred at the cost of insulin-stimulated CD36 translocation (Supplemental Figure 4C and Figure 4D). Additionally, v-ATPase inhibition caused a $>40 \%$ decrease of both insulin-stimulated Akt phosphorylation (Supplemental Figure 4, D and E and Figure 4E) and insulin-stimulated S6 phosphorylation (Supplemental Figure 4, E and $\mathrm{F}$ and Figure $4 \mathrm{~F}$ ). As a positive control, high-palmitate treatment caused $>80 \%$ inhibition of both insulin-stimulated Akt and S6 phosphorylation (Figure 4, E and F). Additionally, in primary cardiomyocytes, v-ATPase inhibition via long-term Bafilomycin-A treatment caused insulin resistance (Supplemental Figure 4, G-N). Moreover, v-ATPase inhibition caused contractile dysfunction, as evident from the $64 \%$ decrease in sarcomere shortening (Figure 4G). Yet, both the contraction acceleration time and the duration of relaxation remained unaffected by Bafilomycin-A (Figure 4, H and I). High-palmitate treatment also induced a decrease of sarcomere shortening of similar magnitude without changes in contraction kinetics (Figure 4, G-I). Taken together, direct v-ATPase inhibition causes increased CD36 translocation to the sarcolemma, insulin resistance and contractile dysfunction.

The development of insulin resistance induced by $V$-ATPase inhibition requires CD36.

To investigate whether increased CD36-mediated LCFA uptake is mandatory for the development of insulin resistance induced by v-ATPase inhibition, CD36 expression was silenced in HL-1 cardiomyocytes using siRNA (knockdown efficiency $50 \pm 11 \%(\mathrm{n}=3$ ); representative blot shown in Supplemental Figure 5A). As expected, CD36 silencing attenuated or blocked the development of the main features of high-palmitate induced insulin resistance, such as increased basal LCFA uptake and of losses of insulin-stimulated LCFA uptake, glucose uptake and Akt and S6 phosphorylation (Figure 5, A-D and Supplemental Figure 5, B-F). In the low-palmitate condition, none of the parameters indicate the development of insulin resistance, whereas long-term Bafilomycin-A treatment in this condition elicits changes similar to those found during high-palmitate culturing (Figure 5, A-E). Importantly, CD36 silencing fully prevented the Bafilomycin-A induced changes in LCFA uptake and insulin signaling (Figure 5, A-D and Supplemental Figure 5, $\mathrm{B}-\mathrm{F})$, therefore disclosing the requirement of CD36 for the development of insulin 
(A)

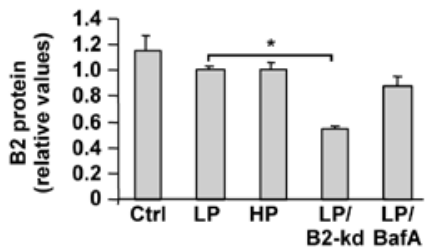

(C)

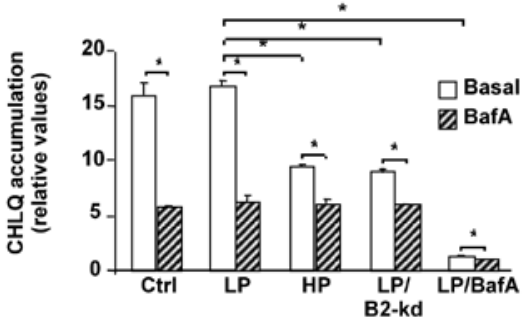

(E)

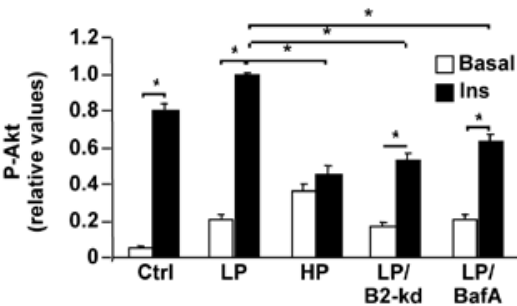

(G)

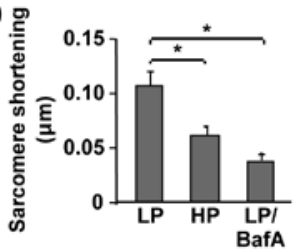

(H)
(B)

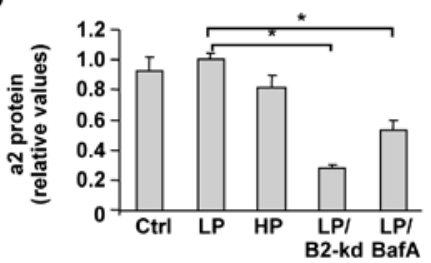

(D)

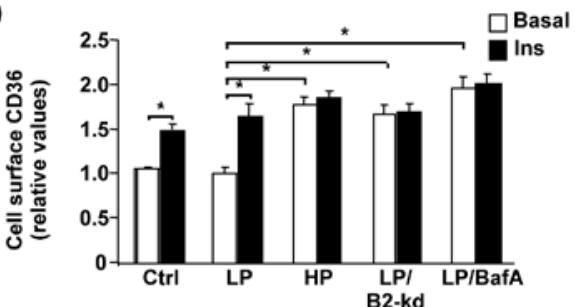

(F)
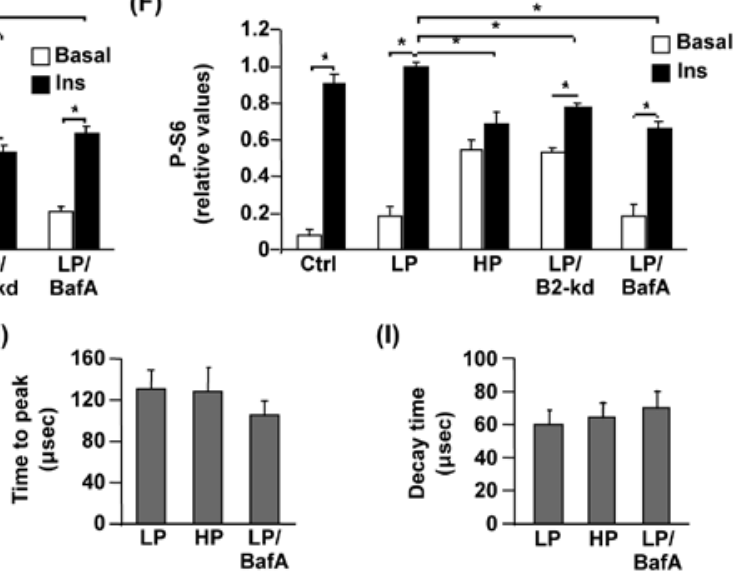

(I)

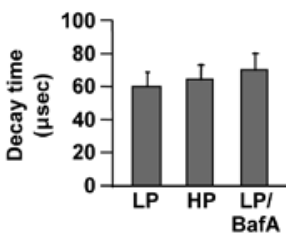

Figure 4. Direct Inhibition of V-ATPase Causes Insulin Resistance and Contractile Dysfunction in Cardiomyocytes. (A-F) HL-1 cardiomyocytes (HL-1) were transfected with negative control scrambled siRNA or with siRNA targeting v-ATPase B2 subunit mRNA (B2-kd). $32 \mathrm{~h}$ after transfection, cells were cultured under control condition (Ctrl), or with low palmitate (LP), high palmitate (HP) or LP enriched with $100 \mathrm{nM}$ Bafilomycin-A (LP/BafA) for $16 \mathrm{~h}$. (A,B) Protein expression of v-ATPase a2 $(n=4-5)$ and B2-subunits ( $n=6-7)$. (C) Chloroquine (CHLQ) accumulation in cardiomyocytes treated without/with $100 \mathrm{nM}$ BafA for $25 \mathrm{~min}(\mathrm{n}=4)$. (D-F) Sarcolemmal CD36 content $(\mathrm{n}=6)$ and phosphorylation of Akt (P-Akt; $n=8-11$ ) and S6 (P-S6; $n=4-9)$ in cardiomyocytes treated without/with $100 \mathrm{nM}$ insulin (Ins) for $30 \mathrm{~min}$. (G-I) Rat cardiomyocytes were incubated in either LP, HP, or LP/BafA for $25 \mathrm{~h}$. Several parameters of contraction were determined upon $1 \mathrm{~Hz}$ electrostimulation: (G) sarcomere shortening; (H) time to peak; (I) decay time ( $\mathrm{n}=4$; imaging of 5 cells/condition). Values are displayed as mean \pm SEM. ${ }^{*} \mathrm{p}<0.05$; N.S., not significant. Data were normalized to LP $(\mathbf{A}, \mathbf{B}), \mathrm{LP} / \mathrm{BafA}$ (C), LP/Basal (D), or to LP/Ins (E,F). 
resistance resulting from v-ATPase inhibition. CD36 silencing also fully prevented the high palmitate-induced loss of Chloroquine accumulation (Figure 5E and Supplemental Figure 5G), thus indicating that CD36-mediated LCFA uptake is responsible for v-ATPase inhibition. These findings that are based on CD36 inhibition in HL1-cardiomyocytes were further confirmed in primary cardiomyocytes, in which an immunological CD36 blockade via CD36 monoclonal antibody prevented lipid accumulation and loss of contractility and endosomal function (Figure 5, F-H). Thus, CD36-mediated LCFA uptake is required for $\mathrm{v}$-ATPase inhibition and the subsequent development of insulin resistance and contractile dysfunction.

\section{Human cardiomyocytes display reduced V-ATPase function upon lipid oversupply.}

To study whether v-ATPase inhibition, as observed in rodent cardiomyocytes upon lipid oversupply, also occurs in human cardiomyocytes, we used human induced pluripotent stem cells (iPSC) differentiated into cardiomyocytes (iPSC-CM). 14 Days after the start of the cardiomyocyte differentiation protocol, $>30 \%$ of the cells displayed spontaneous contractions, and the cardio-specific proteins myosin heavy chain ( $\alpha$ isoform; MYH6) and cardiac troponin T (TNNT2) were upregulated by 4.5 and 2.8-fold respectively (Supplemental Figure 6D). Similar to rodent cardiomyocytes (Figures 1-5), iPSC-CM developed the main features of insulin resistance upon high-palmitate culturing: loss of insulin-stimulated LCFA and glucose uptake and increased basal LCFA uptake (Figure 6, A and B and Supplemental Figure 6, A and B), as well as loss of insulin-stimulated Akt and S6 phosphorylation (Figure 6C). Moreover, iPSC-CM cultured with high-palmitate display loss of Chloroquine accumulation (Figure 6D and Supplemental Figure 6C). Therefore, the mechanism of lipid-induced v-ATPase inhibition leading to insulin resistance likely is conserved between species and thus applicable to the human heart.

Lipid-induced V-ATPase inhibition Is caused by disassembly of the $V_{0} / V_{1}$ subcomplexes.

In earlier studies in yeast, reversible disassembly of v-ATPase into its two sub-complexes $\mathrm{V}_{0}$ and $\mathrm{V}_{1}$ was described as a main mechanism of regulation of this protein $[10,18]$. Thus, HL-1 cardiomyocytes were cultured in high-palmitate and control media, subsequently lysed in the presence of mild detergents, and used for immunoprecipitation with antibodies against the $\mathrm{a} 2$ or B2 subunits of $\mathrm{v}$-ATPase. To assess the degree of assembly, we exploited that a2 is part of the $\mathrm{V}_{0}$ sub-complex and that $\mathrm{B} 2$ is part of the $\mathrm{V}_{1}$ sub-complex. For both immunoprecipitations, we observed that under high-palmitate culturing conditions, the degree of co-immunoprecipitation with the other subunit was markedly lower than under basal conditions (Figure 7A), which indicates $\mathrm{V}_{0} / \mathrm{V}_{1}$ disassembly. We also applied subcellular fractionation to study the influence of LCFA oversupply on the v-ATPase $\mathrm{V}_{0} / \mathrm{V}_{1}$ assembly status. HL-1 cardiomyocytes were fractionated into subcellular mem- 
(A)

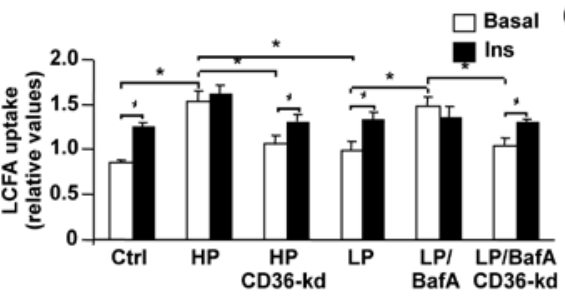

(C)

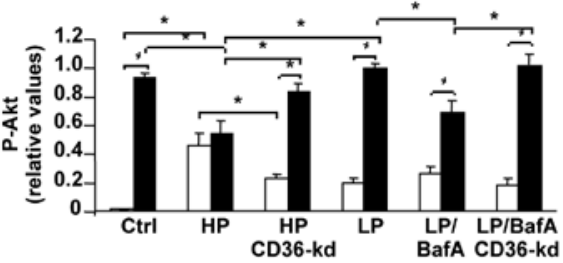

(E)

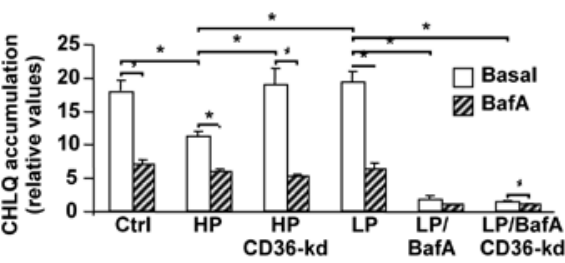

(F)

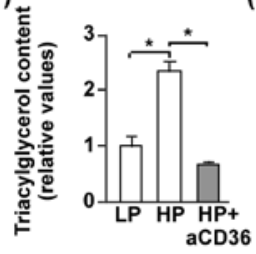

(G)

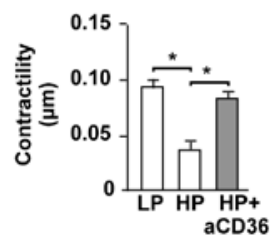

(B)

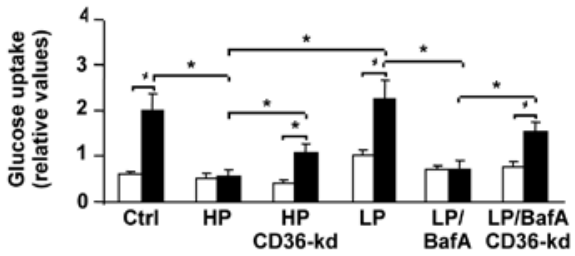

(D)

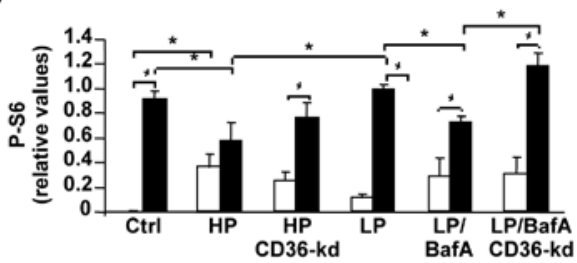

Figure 5. Direct V-ATPase Inhibition Requires CD36 to Develop Insulin Resistance and Contractile Dysfunction. (A-E) HL-1 cardiomyocytes (HL-1) were transfected with negative control scrambled siRNA or siRNA targeting CD36 mRNA (CD36-kd). $32 \mathrm{~h}$ after transfection, cells were treated either with control condition (Ctrl), high palmitate (HP), low palmitate (LP), or LP enriched with $100 \mathrm{nM}$ Bafilomycin-A (LP/BafA) for $16 \mathrm{~h}$. (A-D) LCFA ( $\mathrm{n}=4-5)$ and glucose $(\mathrm{n}=4-6)$ uptake and phosphorylation of Akt (P-Akt; $n=6)$ and S6 (P-S6; n=5-6) in HL-1 treated without/with $100 \mathrm{nM}$ insulin (Ins) for $30 \mathrm{~min}$. (E) Chloroquine (CHLQ) accumulation in HL-1 treated without/with $100 \mathrm{nM}$ BafA for $25 \mathrm{~min}(\mathrm{n}=6)$. (F-H) Rat cardiomyocytes were incubated for $48 \mathrm{~h}$ with either low palmitate (LP), high palmitate (HP), or HP with addition of $0.83 \mu \mathrm{g} / \mathrm{ml}$ anti-CD36-clone 63. (F) Triacylglycerol content $(\mathrm{n}=5)$. (G) Sarcomere shortening upon $1 \mathrm{~Hz}$ electrostimulation $(\mathrm{n}=5)$. (H) CHLQ accumulation in cardiomyocytes treated without/with $100 \mathrm{nM} \mathrm{BafA}(25 \mathrm{~min})(\mathrm{n}=3)$. Values are displayed as mean \pm SEM. " $<<0.05$; N.S., not significant. Data were normalized to LP/Basal (A,B), LP/Ins (C,D), LP/BafA (E) or to LP $(\mathbf{F}, \mathrm{H})$. 
(A)

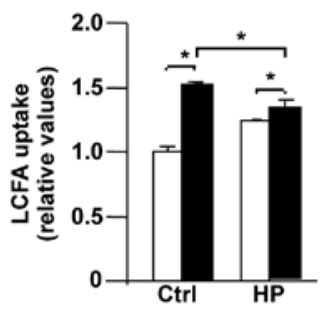

(B)

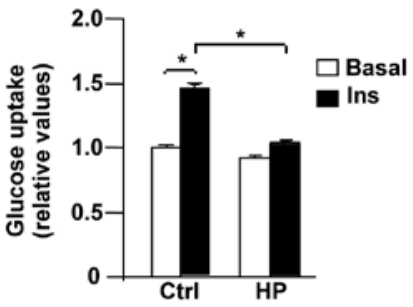

(C)

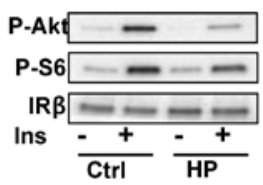

(D)

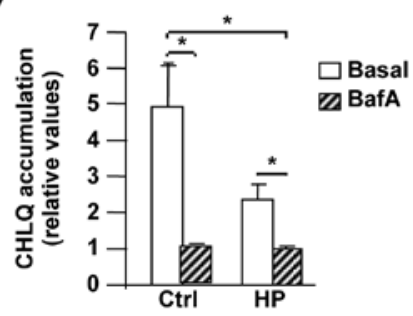

Figure 6. V-ATPase Inhibition Replicates in Human Induced Pluripotent Stem Cell-derived Cardiomyocytes upon Lipid Overload. Human iPSC-derived cardiomyocytes (iPSC-CM) were cultured for $16 \mathrm{~h}$ in control (Ctrl) or HP medium media. (A-C) LCFA and glucose uptake and phosphorylation of Akt (P-Akt) and S6 (P-S6) in human iPSC-CM treated without/with $100 \mathrm{nM}$ insulin (Ins) for $30 \mathrm{~min}$. Loading control: insulin receptor- $\beta$ (IR $\beta$ ). (D) Chloroquine (CHLQ) accumulation in human iPSC-CM treated without/with $100 \mathrm{nM}$ Bafilomycin-A (BafA) for 25 min. Values are displayed as mean $\pm \operatorname{SEM}(n=3)$. ${ }^{*} \mathrm{p}<0.05$; N.S., not significant. Data were normalized to Ctrl/Basal (A,B) or to Ctrl/BafA (D).

branes and cytoplasm. The a2 subunit, as indicator of the membrane-bound $\mathrm{V}_{0}$ sub-complex, was found predominantly in the membrane fraction (Figure 7B), thus confirming the suitability of this fractionation method for studies of v-ATPase disassembly. As part of the soluble $\mathrm{V}_{1}$ sub-complex, $\mathrm{B} 2$ markedly shifted from the membranous to the cytoplasmic fraction upon high-palmitate culturing versus control condition (Figure 7B). Together, these findings strongly indicate that lipid overexposure inhibits $v$-ATPase by $\mathrm{V}_{0} / \mathrm{V}_{1}$ disassembly.

\section{Discussion}

The obtained results lead us to formulate the following main conclusions. (1) Upon lipid-overload in cardiomyocytes, v-ATPase activity decreases by $\mathrm{V}_{0} / \mathrm{V}_{1}$ disassembly, leading to endosomal alkalinization, and thereafter to the onset of insulin resistance. (2) V-ATPase inhibition requires CD36-mediated lipid uptake to cause insulin resistance and contractile dysfunction. These aspects are further discussed below.

Lipid-induced V-ATPase inhibition precedes the onset of insulin resistance.

Subjecting rats to a high-fat diet regime leads to increased CD36 translocation to the 
(A)

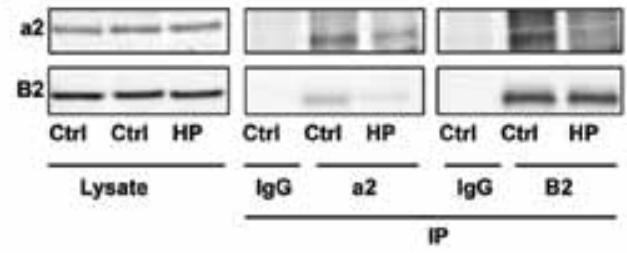

(C)

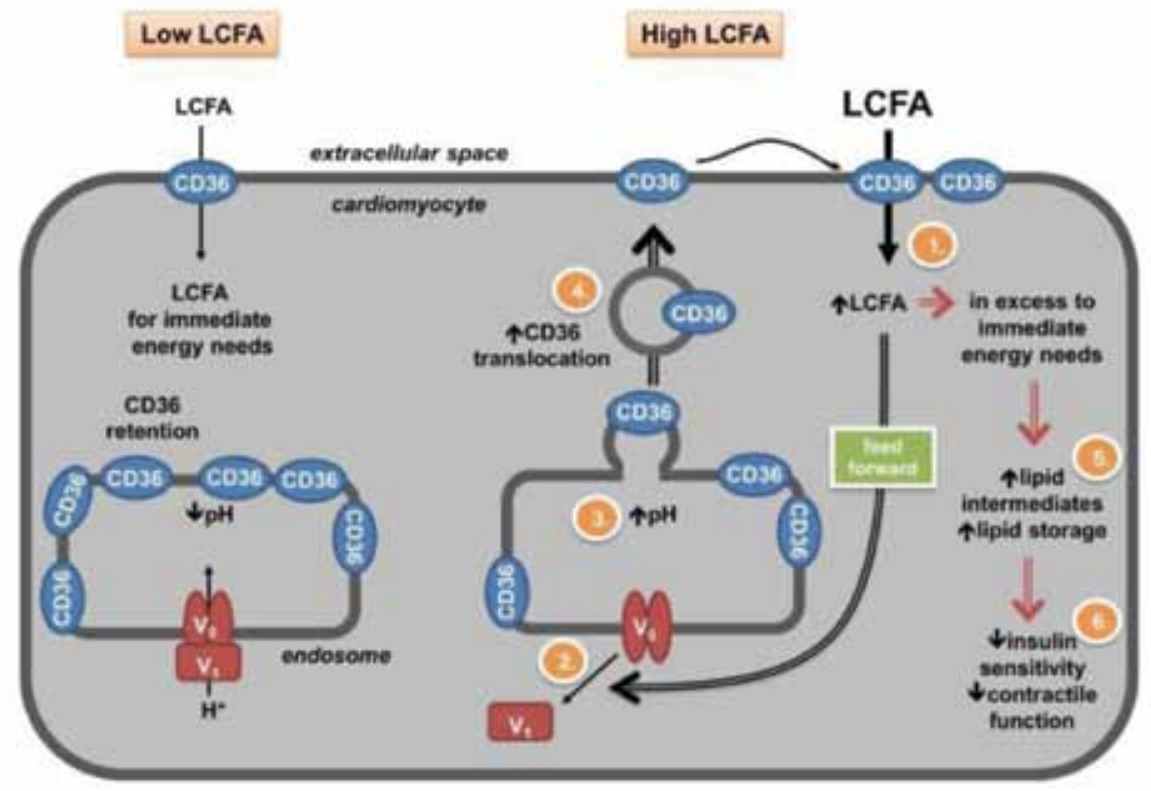

Figure 7. Mechanism and Scheme of Lipid-induced V-ATPase Inhibition: Consequences for Insulin Sensitivity and Contractile Function. HL-1 cardiomyocytes were cultured for $16 \mathrm{~h}$ in control medium (Ctrl) or with high palmitate (HP), and subsequently used for (A) immunoprecipitation (IP) or (B) fractionation. (A) Content of v-ATPase subunits a2 and B2 in cell lysates and in IPs using control IgG, or antibodies capturing a2 and B2 $(n=3-4)$. (B) Content of a2 and B2 in cytoplasmic fraction (C) and membrane fraction (M). Glyceraldehyde 3-phosphate dehydrogenase (GAPDH; loading control for C); caveolin-3 (Cav3; loading control for $\mathrm{M})$ ( $\mathrm{n}=3-4)$. Immunoblots are representative results. (C) Schematic presentation of LCFA-induced alterations leading to cardiac contractile dysfunction. When LCFA supply is low, CD36 is primarily found in endosomes. Furthermore, the v-ATPase $\mathrm{V}_{0}$ sub-complex, which is integral to the endosomal membrane, is assembled with the cytosolic $\mathrm{V}_{1}$ sub-complex allowing for acidification of the endosomal lumen. In this situation, the available LCFA is metabolized to meet the immediate energy demand. Elevated extracellular LCFA supply triggers a series of events: (1) Increased CD36-mediated LCFA uptake results in elevated intramyocellular LCFA levels. (2) LCFA causes the $V_{1}$ and $V_{0}$ sub-complexes to dissociate. Therefore, $\mathrm{V}_{1}$ is shifted towards the cytoplasm. (3) The disassembly of v-ATPase leads to endosomal alkalinization. (4) Increased endosomal $\mathrm{pH}$ triggers the translocation of CD36 vesicles to the sarcolemma. Upon chronic lipid oversupply, where LCFA uptake surpasses the metabolic needs, further processes are set in motion: (5) The lipid-induced increase in sarcolemmal CD36 feeds forward to further increased LCFA uptake and progressive accumulation of lipid intermediates. (6) The lipid overload culminates into loss of insulin sensitivity and contractile function. 
sarcolemma, chronically increased LCFA uptake, lipid accumulation, impaired insulin signaling, impaired insulin-stimulated glucose uptake, cardiac morphological changes and, finally, impaired contractile function [6]. CD36 is a key factor, as CD36-KO mice were protected against the development of lipid-induced cardiomyopathy $[16,19]$. In the present study, the lipid-induced manifestations occurred in conjunction with v-ATPase inhibition in cardiomyocytes from high-fat diet rats (Figure 1), lipid-overexposed primary rat cardiomyocytes (Figure 2), mouse-derived HL-1 cardiomyocytes Supplemental Figure 2) and human iPSC-derived cardiomyocytes (Figure 7). Importantly, upon lipid overload in vitro, we observed a similar degree of v-ATPase inhibition as displayed in vivo, i.e., $\approx 50 \%$ in primary cardiomyocytes and HL- 1 cardiomyocytes (Figure 1 and S1) and by $\approx 70 \%$ in human iPSC-CM (Figure 6). Given that Chloroquine is a divalent weak base, it will accumulate as square function of endosomal $\mathrm{pH}$ changes [17]. Assuming that the intraluminal $\mathrm{pH}$ of fully functional endosomes is 5.5 [20], while that of endosomes with complete loss of v-ATPase activity will be at cytosolic pH 7.0, we estimate an elevation of 0.4-0.7 $\mathrm{pH}$ units (i.e., from $\mathrm{pH} 5.5$ to 5.9-6.2; using eq. 1) in rodent and human cardiomyocytes, respectively, upon lipid oversupply.

Equation 1. $\Delta \mathrm{pH}=\sqrt{ } 100(\%)-\sqrt{ }(100-\mathrm{x})(\%)) /(\sqrt{ } 100(\%) *(7.0-5.5)$

$\mathrm{x}$ : \% decrease of Chloroquine accumulation

Our chronological assessment of the hallmark events of lipid-induced insulin resistance in cardiomyocytes in relation to the onset of endosomal alkalinization revealed that v-ATPase inhibition is an early event already occurring within $1 \mathrm{~h}$ after high-palmitate addition that is simultaneous with a 1.5-fold increase in myocellular LCFA uptake (Figures 3 and Supplemental Figure 3). Since we previously observed that Bafilomycin-A treatment already induces CD36 translocation and increases LCFA uptake within minutes [11], it was to be expected that on a resolution scale of hours, both events could not be separated. Interestingly, the rapid increase in basal LCFA uptake upon the start of the high-palmitate treatment occurred at the cost of insulin-stimulated LCFA uptake, which was completely blunted (Figure 3B). Furthermore, high-palmitate-induced CD36 translocation renders the cells insensitive to further insulin-stimulated CD36 translocation (Figure 4D). This observation suggests that the insulin-responsive CD36-containing endosomal subcompartment provides the source of the rapid CD36 translocation upon exposure of cardiomyocytes to excess lipids. Importantly, the impairment of insulin signaling and insulin-stimulated glucose uptake followed much later, i.e., at 14 hours after high-palmitate addition, indicating that v-ATPase inhibition and CD36 translocation precede the highpalmitate-induced insulin resistance. When zooming in on the time course of v-ATPase inhibition, there is a rapid fall in v-ATPase function within the first hour of palmitate addition, which is followed by a second slower phase of further loss until $25 \mathrm{~h}$. The initial 
rapid loss upon lipid oversupply represents $34 \%$ of the total loss in v-ATPase function (Supplemental Figure 3A). Yet, the increase in myocellular LCFA uptake, and likely also the increased CD36 translocation to the sarcolemma, was already maximal after $1 \mathrm{~h}$, implicating that only this 34\% drop in v-ATPase function could be held accountable for increased CD36 translocation. Hence, expulsion of CD36 from the endosomes occurs in response to relatively small increases in endosomal $\mathrm{pH}$ (i.e., $0.3 \mathrm{pH}$ units, according to eq. 1). These present results are furthermore in good agreement with our recent in vivo findings [21], where we observed rapid increases in CD36 translocation to the sarcolemma, myocellular LCFA uptake, accumulation of triacylglycerol and diacylglycerol (2-3 days) and a delayed impairment in insulin signaling and GLUT4 translocation ( $>21$ days). Therefore, the processes that underlie the progression from lipid accumulation into insulin resistance appear to be complex and more time-consuming than the relatively fast occurring v-ATPase inhibition and subsequent CD36-mediated lipid uptake.

\section{$V$-ATPase inhibition requires CD36 to cause insulin resistance and contractile dysfunction.}

We have identified CD36 and v-ATPase as the main actors in lipid-induced lipid uptake, which also co-localize to the same endosomal vesicles (Figure $1 \mathrm{H}$ ). Before turning to the mandatory role of CD36, we first discuss the inhibition of v-ATPase as an intermediary of increased lipid uptake leading to insulin resistance and contractile dysfunction. Direct evidence for a causal role of v-ATPase in lipid-induced insulin resistance in cardiomyocytes was obtained by siRNA-mediated silencing of v-ATPase (in HL-1 cardiomyocytes) and long-term Bafilomycin-A treatment (in HL-1 and primary cardiomyocytes). Both strategies markedly inhibited v-ATPase function, increased CD36 translocation to the sarcolemma, and caused insulin resistance in a similar manner as seen in high-palmitate-treated cardiomyocytes.

With respect to the effects v-ATPase inhibition on contraction mechanics, long-term Bafilomycin-A treatment of cardiomyocytes exposed to low palmitate caused a decrease in sarcomere shortening, the degree of which was similar to the decrease in sarcomere shortening due to high palmitate treatment. Another similarity with high-palmitateinduced contractile dysfunction is that the Bafilomycin-A induced decrease in sarcomere shortening was not accompanied by changes in contraction acceleration or relaxation time, indicating that both treatments have the same specific impact on contractile mechanics. This makes it unlikely that the effect of Bafilomycin-A treatment is due to a general toxic action mechanism. After all, such a general toxic effect would not only decrease the contraction amplitude but also increase the relaxation time, as seen for example by mitochondrial inhibition [22]. Our investigations are the first to causally link $\mathrm{v}$-ATPase inhibition not only to insulin resistance, but also to contractile dysfunction, 
thereby implicating that endosomal $\mathrm{pH}$ increase underlies the development of cardiomyopathy.

Second, CD36-mediated LCFA uptake as a causality of v-ATPase inhibition is discussed. CD36 downregulation prevented the loss in high-palmitate induced v-ATPase inhibition (Figure 5, E and $\mathrm{H}$ ), i.e., pointing to de-activation of v-ATPase as a consequence of CD36-mediated LCFA uptake. As expected, CD36 downregulation also prevented the onset of insulin resistance in high-palmitate treated cardiomyocytes. Treatment with Bafilomycin-A as well as high-palmitate in conjunction with knockdown of CD36 protein expression (Supplemental Figure 5A) effectively prevented increased LCFA uptake (Figure 5B), lipid accumulation (Figure 5F) and insulin resistance (Figure 5, C and D and Supplemental Figure 5, D-F). Notably, the achieved level of 50\% CD36 downregulation adequately counterbalanced the $\approx 70 \%$ increase in CD36 content at the sarcolemma in high palmitate- or Bafilomycin-A-treated cells (Figure 4D). Taken together, we describe a novel v-ATPase-CD36 interplay showing critical involvement in the development of lipid-induced insulin resistance and contractile dysfunction.

Concerning the mechanism by which intracellularly sequestered lipids will inhibit v-ATPase, we speculated that lipids might cause its disassembly into the two subcomplexes, $\mathrm{V}_{0}$ (that will remain integral to the endosomal membrane) and $\mathrm{V}_{1}$ (that will drift away into the cytoplasm). Cycles of assembly/disassembly appeared to be the main mechanism of regulation of $\mathrm{v}$-ATPase activity in yeast [10]. Indeed, using two independent techniques, i.e., immunoprecipitation and subcellular fractionation, we demonstrated that lipids induce the disassembly of $\mathrm{v}$-ATPase and the migration of $\mathrm{V}_{1}$ into the cytoplasm (Figure 7, A and B). Together, this indicates $\mathrm{v}$-ATPase as a possible lipid sensor in cardiomyocytes.

\section{Limitations of the study.}

V-ATPase is not only present in endosomes, but also in other acidic vesicles, most notably lysosomes. Hence, the genetic and pharmacological approaches to inhibit v-ATPase may not only cause endosomal alkalinization, but also a partial lysosomal dysfunction. However, given their main function as organelles involved in degradation of subcellular components, lysosomes would not be expected to contribute to subcellular storage of CD36 and thereby participate in the regulation of LCFA uptake. Still, lysosomal dysfunction could also alter subcellular lipid homeostasis at another level, being the autophagic degradation of triacylglycerols involving lysosomal lipases. Thus, lysosomal $\mathrm{pH}$ elevation could impair the activity of the lysosomal lipases and thereby contribute to lipid accumulation and lipid-induced insulin resistance. Yet, lysosomal lipases likely form a negligible player in total cellular lipid turnover compared to the high degradation capacity of the cytoplasmic lipases ATGL and HSL [23]. 


\section{General conclusions.}

We identify v-ATPase $\mathrm{V}_{0} / \mathrm{V}_{1}$ disassembly as a trigger of increased CD36 translocation, and thus describe a novel function of v-ATPase. Further, CD36-mediated lipid uptake upon lipid supply feeds forward to increased CD36 translocation to the sarcolemma and further increased lipid uptake. Hence, v-ATPase inhibition explains the well-described shift of nutrient uptake preference of cardiomyocytes from glucose to lipids in response to excess lipid availability [3]. Accordingly, chronic lipid oversupply by inhibiting v-ATPase gives rise to a vicious cycle of accelerated myocellular lipid accumulation setting the heart on a road to insulin resistance and contractile dysfunction (Figure 7C). Since the mechanism of lipid-induced v-ATPase inhibition is conserved in human cardiomyocytes, the identification of pharmacological agents that stabilize the assembly of $v$-ATPase $V_{1} / V_{0}$ sub-complexes may be considered as a possible novel strategy to combat lipid-induced cardiomyopathy. 


\section{References}

1. L.S. Szczepaniak, R.G. Victor, L. Orci, R.H. Unger, Forgotten but not gone: the rediscovery of fatty heart, the most common unrecognized disease in America, Circ Res, 101 (2007) 759-767.

2. R. Harmancey, H. Taegtmeyer, The complexities of diabetic cardiomyopathy: lessons from patients and animal models, Curr Diab Rep, 8 (2008) 243-248.

3. J.F. Glatz, J.J. Luiken, A. Bonen, Membrane fatty acid transporters as regulators of lipid metabolism: implications for metabolic disease, Physiol Rev, 90 (2010) 367-417.

4. J.J. Luiken, D.P. Koonen, J. Willems, A. Zorzano, C. Becker, Y. Fischer, N.N. Tandon, G.J. Van Der Vusse, A. Bonen, J.F. Glatz, Insulin stimulates long-chain fatty acid utilization by rat cardiac myocytes through cellular redistribution of FAT/ CD36, Diabetes, 51 (2002) 3113-3119.

5. S.L. Coort, D.M. Hasselbaink, D.P. Koonen, J. Willems, W.A. Coumans, A. Chabowski, G.J. van der Vusse, A. Bonen, J.F. Glatz, J.J. Luiken, Enhanced sarcolemmal FAT/CD36 content and triacylglycerol storage in cardiac myocytes from obese zucker rats, Diabetes, 53 (2004) 1655-1663.

6. D.M. Ouwens, M. Diamant, M. Fodor, D.D. Habets, M.M. Pelsers, M. El Hasnaoui, Z.C. Dang, C.E. van den Brom, R. Vlasblom, A. Rietdijk, C. Boer, S.L. Coort, J.F. Glatz, J.J. Luiken, Cardiac contractile dysfunction in insulin-resistant rats fed a high-fat diet is associated with elevated CD36-mediated fatty acid uptake and esterification, Diabetologia, 50 (2007) 1938-1948.

7. D.B. Savage, K.F. Petersen, G.I. Shulman, Disordered lipid metabolism and the pathogenesis of insulin resistance, Physiol Rev, 87 (2007) 507-520.

8. V. Marshansky, M. Futai, The V-type H+-ATPase in vesicular trafficking: targeting, regulation and function, Curr Opin Cell Biol, 20 (2008) 415-426.

9. B. Ma, Y. Xiang, L. An, Structural bases of physiological functions and roles of the vacuolar H(+)-ATPase, Cell Signal, 23 (2011) 1244-1256.

10. P.M.Kane, argeting reversible disassembly as a mechanism of controlling V-ATPase activity, Curr Protein Pept Sci, 13 (2012) 117-123.

11. L.K. Steinbusch, W. Wijnen, R.W. Schwenk, W.A. Coumans, N.T. Hoebers, D.M. Ouwens, W.A. Coumans, N.T. Hoebers, M. Diamant, A. Bonen, J.F. Glatz, J.J. Luiken, Differential regulation of cardiac glucose and fatty acid uptake by endosomal $\mathrm{pH}$ and actin filaments, Am J Physiol Cell Physiol, 298 (2010) C1549-1559.
12. J.J. Luiken, F.A. van Nieuwenhoven, G. America, G.J. van der Vusse, J.F. Glatz, Uptake and metabolism of palmitate by isolated cardiac myocytes from adult rats: involvement of sarcolemmal proteins, J Lipid Res, 38 (1997) 745758.

13. Y. Angin, L.K. Steinbusch, P.J. Simons, S. Greulich, N.T. Hoebers, K. Douma, M.A. van Zandvoort, W.A. Coumans, W. Wijnen, M. Diamant, D.M. Ouwens, J.F. Glatz, J.J. Luiken, CD36 inhibition prevents lipid accumulation and contractile dysfunction in rat cardiomyocytes, Biochem J, 448 (2012) 43-53.

14. R.W. Schwenk, E. Dirkx, W.A. Coumans, A. Bonen, A. Klip, J.F. Glatz, J.J. Luiken, Requirement for distinct vesicle-associated membrane proteins in insulin- and AMPactivated protein kinase (AMPK)-induced translocation of GLUT4 and CD36 in cultured cardiomyocytes, Diabetologia, 53 (2010) 22092219.

15. R.W. Schwenk, Y. Angin, L.K. Steinbusch, E. Dirkx, N. Hoebers, W.A. Coumans, A. Bonen, J.L. Broers, G.J. van Eys, J.F. Glatz, J.J. Luiken, Overexpression of vesicle-associated membrane protein (VAMP) 3, but not VAMP2, protects glucose transporter (GLUT) 4 protein translocation in an in vitro model of cardiac insulin resistance, J Biol Chem, 287 (2012) 37530-37539.

16. L.K. Steinbusch, J.J. Luiken, R. Vlasblom, A. Chabowski, N.T. Hoebers, W.A. Coumans, I.O. Vroegrijk, P.J. Voshol, D.M. Ouwens, J.F. Glatz, M. Diamant, Absence of fatty acid transporter CD36 protects against Western-type diet-related cardiac dysfunction following pressure overload in mice, Am J Physiol Endocrinol Metab, 301 (2011) E618-627.

17. J.J. Luiken, J.M. Aerts, A.J. Meijer, The role of the intralysosomal $\mathrm{pH}$ in the control of autophagic proteolytic flux in rat hepatocytes, Eur J Biochem, 235 (1996) 564-573.

18. S. Bond, M. Forgac, The Ras/cAMP/protein kinase A pathway regulates glucose-dependent assembly of the vacuolar $(\mathrm{H}+)$-ATPase in yeast, $J$ Biol Chem, 283 (2008) 36513-36521.

19. J. Yang, N. Sambandam, X. Han, R.W. Gross, M. Courtois, A. Kovacs, M. Febbraio, B.N. Finck, D.P. Kelly, CD36 deficiency rescues lipotoxic cardiomyopathy, Circ Res, 100 (2007) 1208-1217.

20. S.L. Rybak, R.F. Murphy, Primary cell cultures from murine kidney and heart differ in endosomal pH, J Cell Physiol, 176 (1998) 216222. 
21. A. Bonen, S.S. Jain, L.A. Snook, X.X. Han, Y. Yoshida, K.H. Buddo, J.S. Lally, E.D. Pask, S. Paglialunga, M.S. Beaudoin, J.F. Glatz, J.J. Luiken, E. Harasim, D.C. Wright, A. Chabowski, G.P. Holloway, Extremely rapid increase in fatty acid transport and intramyocellular lipid accumulation but markedly delayed insulin resistance after high fat feeding in rats, Diabetologia, 58 (2015) 2381-2391.

22. S. Kettlewell, P. Cabrero, S.A. Nicklin, J.A. Dow, S. Davies, G.L. Smith, Changes of intramitochondrial $\mathrm{Ca} 2+$ in adult ventricular cardiomyocytes examined using a novel fluorescent $\mathrm{Ca} 2+$ indicator targeted to mitochondria, J Mol Cell Cardiol, 46 (2009) 891901.
23. M. Goeritzer, N. Vujic, S. Schlager, P.G. Chandak, M. Korbelius, B. Gottschalk, C. Leopold, S. Obrowsky, S. Rainer, P. Doddapattar, E. Aflaki, M. Wegscheider, V. Sachdev, W.F. Graier, D. Kolb, B. Radovic, D. Kratky, Active autophagy but not lipophagy in macrophages with defective lipolysis, Biochim Biophys Acta, 1851 (2015) 1304-1316. 
(A)

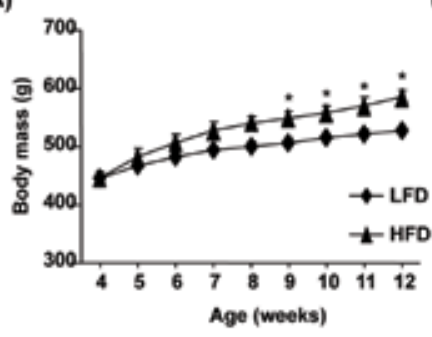

(D)

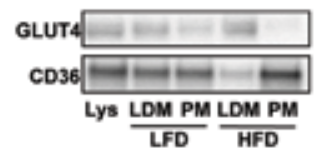

(F)
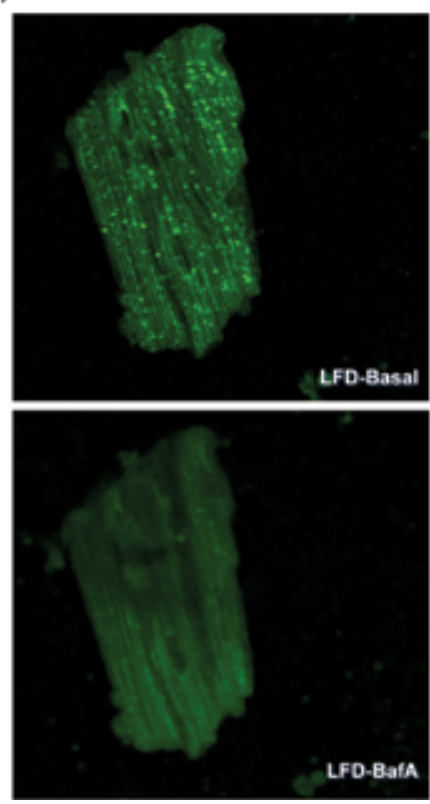

(B)

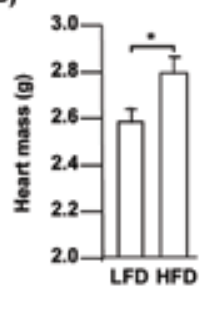

(C)

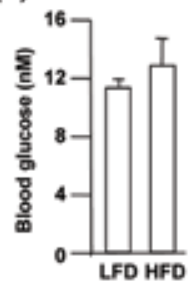

(E)
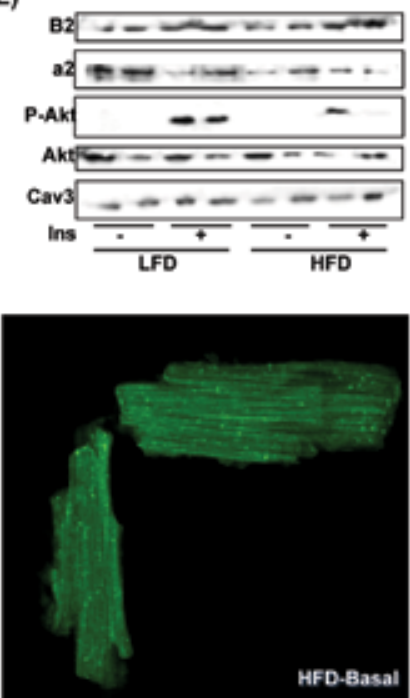

HFD-Basal

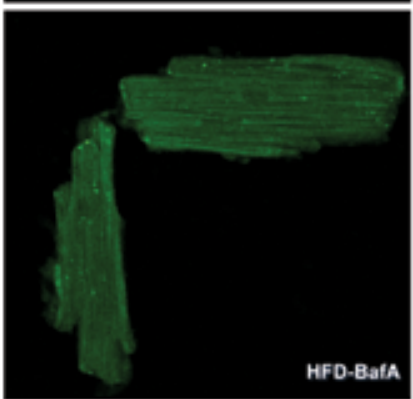

Figure S1: Related to Figure 1

Lewis rats were subjected to low-fat diet (LFD) or high-fat diet (HFD) for 7 weeks. (A) Each week, body mass measurements were made (LF: $n=7 ; H F: n=7$ ), and at the end of the diet regime, (B) heart mass $(n=7)$ and $(C)$ blood glucose concentration $(n=6)$ were measured. Then, cardiomyocytes were isolated for (D) measurement of localization of substrate transporters upon subcellular fractionation yielding plasma membrane fraction (PM) and low density microsomal fraction (LDM) (representative blots are shown; quantification in Figure $1 \mathbf{A}$ and $\mathbf{B}$ ); metabolic measurements upon incubation with radiolabeled substrates (see Figure 1C-D); (E) Western blot analysis of expression of v-ATPase subunits a2 and B2, and phosphorylation of Akt (upon 15 min pre-incubation with $100 \mathrm{nM}$ insulin (Ins)). Total Akt expression and that of caveolin-3 (Cav3) serve as loading controls. Representative blots are shown. Finally, (F) cardiomyocytes were used for lysosensor staining (quantification in Figure 1I). In contrast to HFD, LFD cardiomyocytes displayed a particulate staining pattern, which was sensitive to shortterm (15 min) treatment with $100 \mathrm{nM}$ Bafilomycin-A (BafA). Quantification is shown in Figure 1I. Lys: cardiomyocyte lysate. Values are displayed as mean \pm SEM. ${ }^{*}<0.05$; N.S., not significant. 
(A)

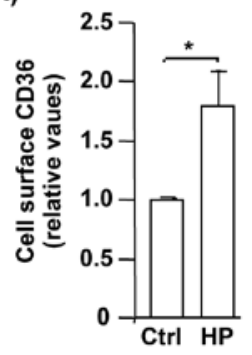

(E)

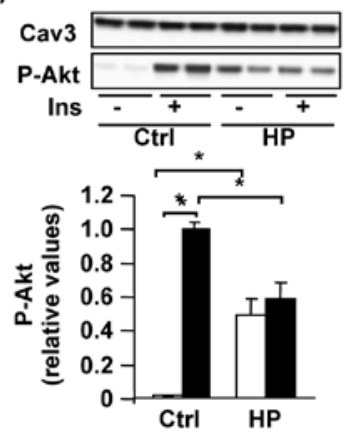

(B)

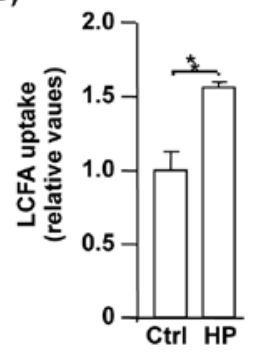

(C)

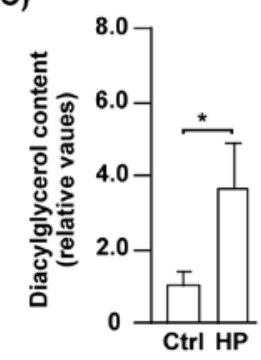

(D)

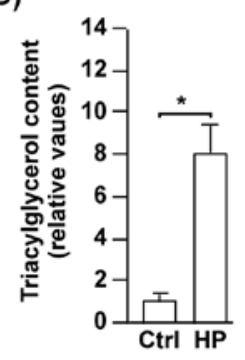

(F)

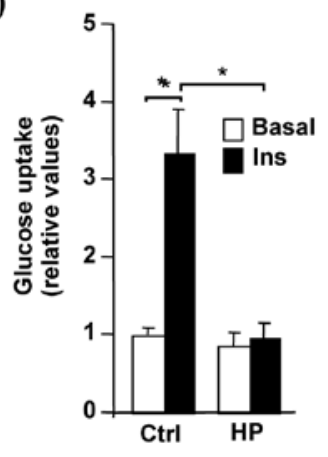

(G)

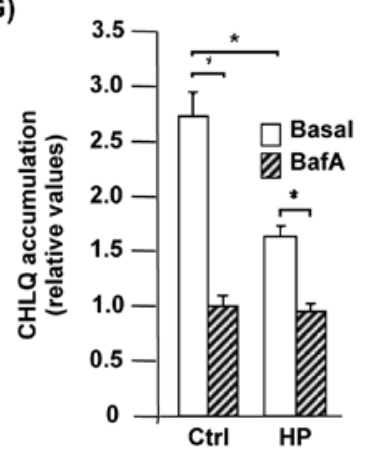

Figure S2: Related to Figure 2

HL-1 cardiomyocytes (HL-1) were cultured in control condition (Ctrl), or with high-palmitate (HP) for $16 \mathrm{~h}$, and thereafter were used for measurement of (A) surface CD36 content; (B) LCFA uptake; intracellular contents of (C) diacylglycerol (DAG) and (D) triacylglycerol (TAG); (E) Akt phosphorylation and (F) glucose uptake (upon $30 \mathrm{~min}$ pre-incubation without/with $100 \mathrm{nM}$ insulin (Ins)) and (G) Chloroquine (CHLQ) accumulation (upon $25 \mathrm{~min}$ pre-incubation without/with 100 $n M$ Bafilomycin-A (BafA)). Values are displayed as mean $\pm \operatorname{SEM}(n=3) .{ }^{*} p<0.05$; 
(A)

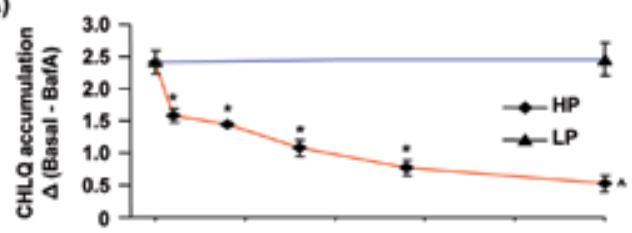

(B)

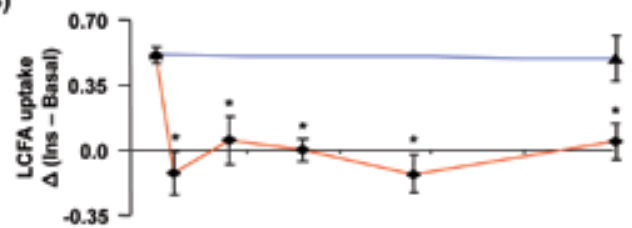

(C)

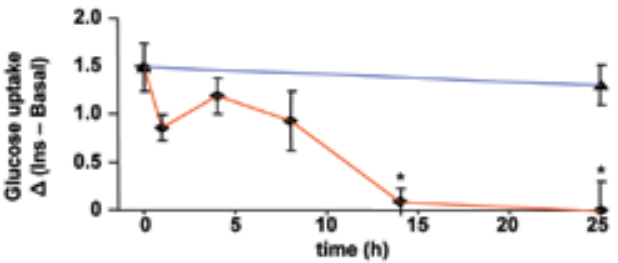

(D)

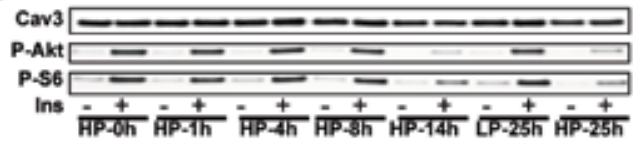

(E)

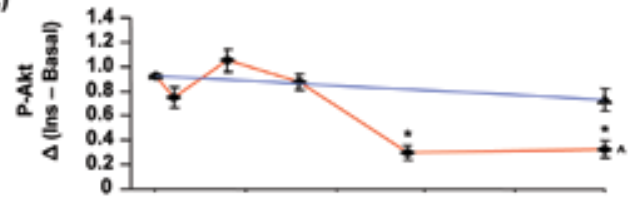

(F)

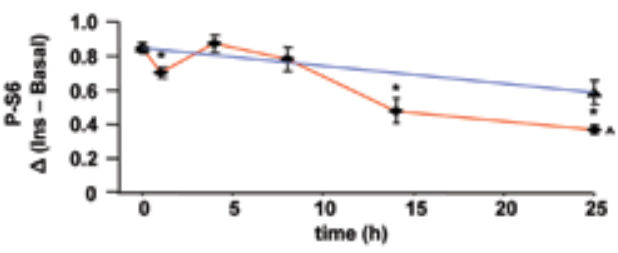

Figure S3. Related to Figure 3

Rat cardiomyocytes were cultured with either low palmitate (LP) or high palmitate (HP) for $0,1,3,8$, 15 , and maximum to $25 \mathrm{~h}$. At each time point, cardiomyocytes were incubated without/with $100 \mathrm{nM}$ Bafilomycin-A (BafA) for $25 \mathrm{~min}$ prior to $30 \mathrm{~min}$ incubation with $\left[{ }^{3} \mathrm{H}\right]$ Chloroquine for assessment of v-ATPase function (see figure 3A). (A) Chloroquine (CHLQ) accumulation is expressed as difference between basal and BafA-treated for each condition $(\triangle \mathrm{Basal}-\mathrm{BafA})$. These data are from the same set of experiments as the data displayed in Figure 3A. (B-E) In parallel at each time point, cardiomyocytes were incubated without/with $100 \mathrm{nM}$ insulin (Ins) for $15 \mathrm{~min}$ prior to execution of $(\mathbf{B})\left[{ }^{14} \mathrm{C}\right]$ palmitate, or (C) $\left[{ }^{3} \mathrm{H}\right]$ glucose uptake studies, or (D-F) Western blot analysis of Akt phosphorylation (P-Akt) and S6 phosphorylation (P-S6) (D: representative Western blots; E and F: quantifications). The effect of insulin is expressed as difference between acute insulin-stimulated and basal for each condition ( $\Delta$ InsBasal). These data are from the same set of experiments as the data from Figure 3B-E. Values are displayed as mean \pm SEM. ${ }^{*}$ p $<0.05$; N.S., not significant. 
(A)

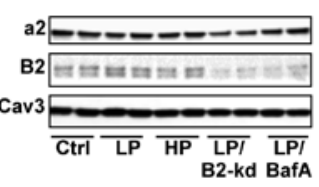

(D)

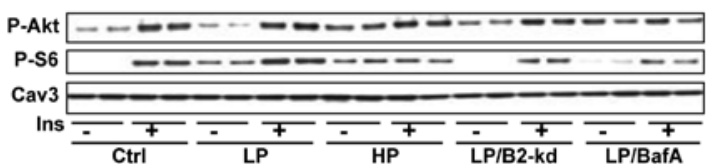

(F)

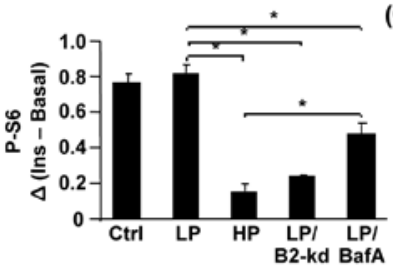

(I)

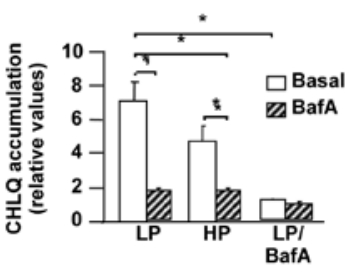

(L)

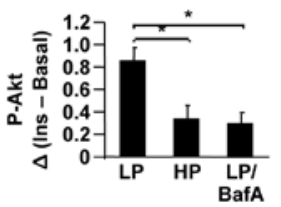

(B)

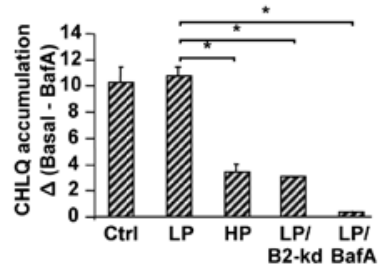

(J)

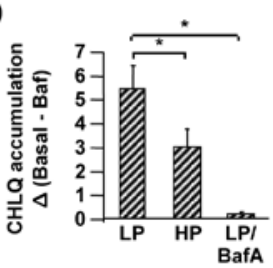

(M)

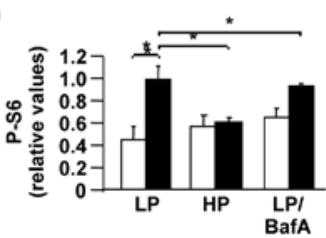

(E)
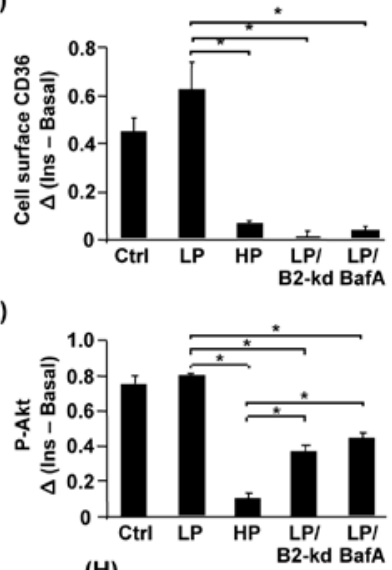

(H)
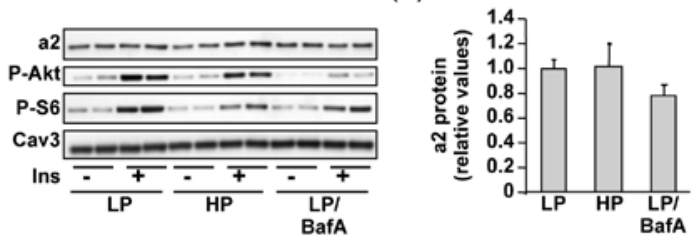

(K)

)

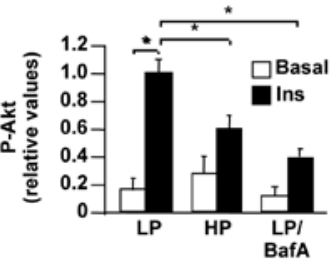

(N)

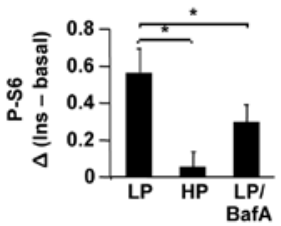

Figure S4: Related to Figure 4

(A-F) HL-1 cardiomyocytes (HL-1) were transfected with negative control scrambled siRNA or with siRNA targeting v-ATPase B2 subunit mRNA (B2-kd). $32 \mathrm{~h}$ after transfection, cells were cultured under control condition (Ctrl), or with low palmitate (LP), high palmitate (HP) or LP enriched with $100 \mathrm{nM}$ Bafilomycin-A (LP/Baf) for $16 \mathrm{~h}$, and thereafter used for further analyses. (A) Whole cell lysates were used for Western blot analysis of v-ATPase a2 and B2. Representative Western blots are shown (caveolin-3 (Cav3): loading control; quantification in Figure 4A-B). (B) HL-1 of each condition were further incubated either without/with $100 \mathrm{nM}$ BafA for $25 \mathrm{~min}$ prior to $30 \mathrm{~min}$ incubation with $\left[{ }^{3} \mathrm{H}\right]$ Chloroquine (CHLQ). CHLQ accumulation is expressed as difference between basal and BafAtreated for each condition ( $\triangle \mathrm{Basal}-\mathrm{BafA})$. These data are from the same set of experiments as the data displayed in Figure 4C. (D-F) In parallel, HL-1 were further incubated without/with $200 \mathrm{nM}$ insulin (Ins) for $30 \mathrm{~min}$, and finally used for (C) colorimetric analysis of sarcolemmal CD36 content, or (D-F) Western blot analysis of Akt phosphorylation (P-Akt) and S6 phosphorylation (P-S6) (D: representative Western blots; E and F: quantifications). The effect of insulin is expressed as difference between acute insulin-stimulated and basal for each condition ( $\Delta$ Ins-Basal). These data are from the same set of experiments as the data from Figure 4D-F. (G-N) Rat cardiomyocytes (CM) were incubated in LP, HP, 
or LP/BafA for $25 \mathrm{~h}$. (G) Whole cell lysates were used for Western blot analysis of v-ATPase subunit expression and of phosphorylation of Akt and S6. For the protein phosphorylation experiments, CM were incubated without/with $100 \mathrm{nM}$ Ins for $15 \mathrm{~min}$. Representative Western blots are shown (caveolin-3 (Cav3): loading control). (H) Quantification of a2 expression ( $\mathrm{n}=4$ ) ( $\mathbf{I}$ and $\mathbf{J}$ ) $\mathrm{CM}$ of each condition were further incubated either without/with $100 \mathrm{nM}$ BafA for $25 \mathrm{~min}$ prior to $30 \mathrm{~min}$ incubation with $\left[{ }^{3} \mathrm{H}\right] \mathrm{CHLQ}(\mathrm{n}=6)$. (J) CHLQ accumulation is expressed as difference between basal and BafA-treated for each condition ( $\triangle$ Basal-BafA). (K-N) Quantification of P-Akt $(\mathrm{n}=4)$ and P-S6 $(n=4)$. ( $(\mathbf{L}$ and $\mathbf{N})$ The effect of insulin is expressed as difference between acute insulin-stimulated and basal for each condition ( $\Delta$ Ins-Basal). Values are displayed as mean \pm SEM. "p $<0.05$; N.S., not significant. 
(A)

$\mathrm{CD} 36$

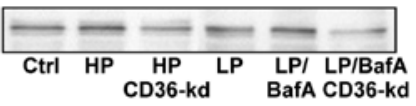

(B)

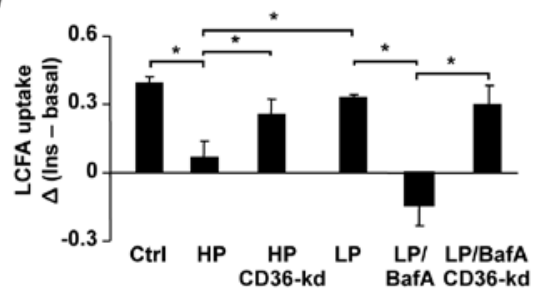

(C)

(D)

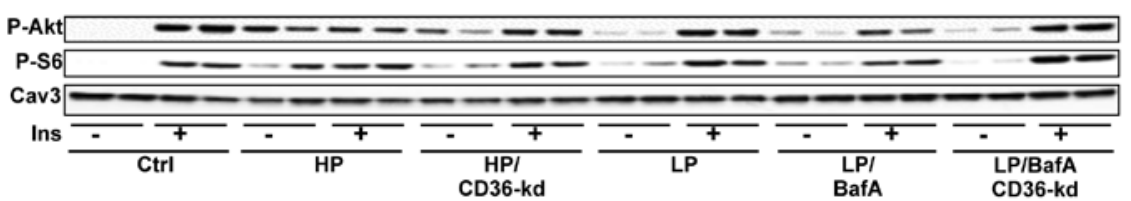

(E)

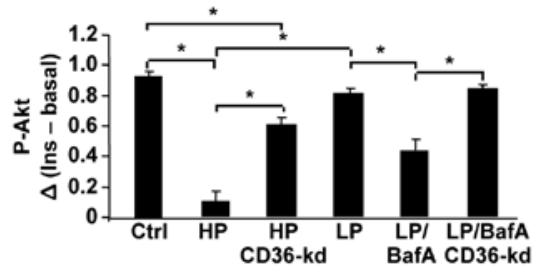

(F)

(G)
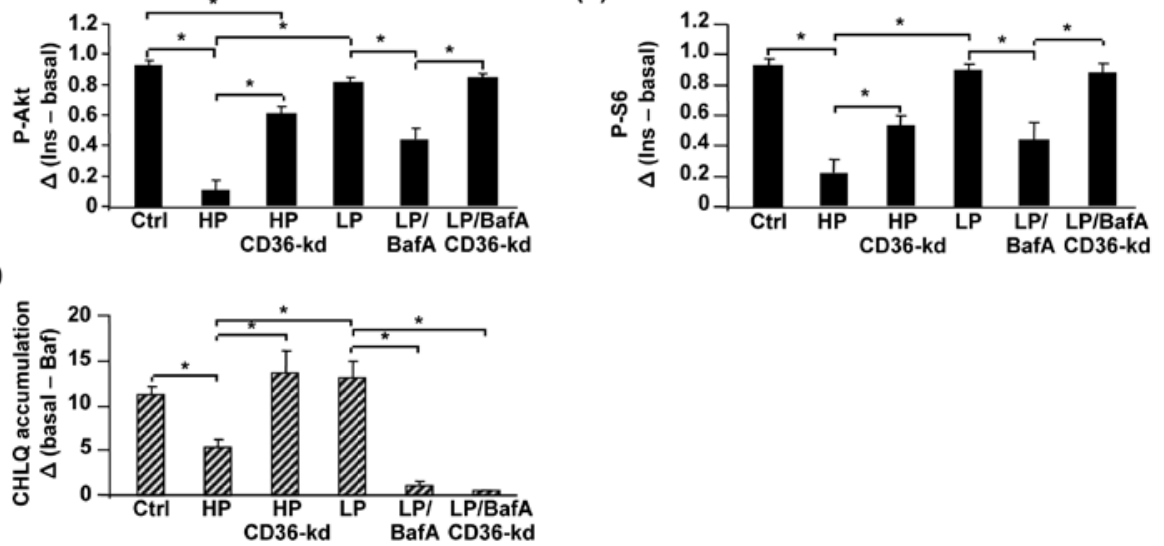

Figure S5: Related to Figure 5

(A-E) HL-1 cardiomyocytes (HL-1) were transfected with negative control scrambled siRNA or with siRNA targeting CD36 mRNA (CD36-kd). $32 \mathrm{~h}$ after transfection, cells were treated either with control condition (Ctrl), high palmitate (HP), low palmitate (LP), or LP enriched with $100 \mathrm{nM}$ Bafilomycin-A (LP/BafA) for $16 \mathrm{~h}$, and used for subsequent incubations and analysis. (A) At each condition, the protein expression level of CD36 was assessed by Western blotting. (B-E) HL-1 were incubated without/with $200 \mathrm{nM}$ insulin (Ins) for $30 \mathrm{~min}$ prior to execution of $(\mathbf{B})\left[{ }^{14} \mathrm{C}\right]$ palmitate and (C) $\left[{ }^{3} \mathrm{H}\right]$ glucose uptake studies, or Western blot analysis of Akt phosphorylation (P-Akt) and S6 phosphorylation (P-S6) (D: representative Western blots; caveolin-3 (Cav3): loading control; E and F: quantifications). The effect of insulin is expressed as difference between acute insulin-stimulated and basal for each condition ( $\triangle$ Ins-Basal). These data are from the same set of experiments as the data from Figure 5A-D. (G) In parallel, at each condition, HL-1 were incubated without/with $100 \mathrm{nM}$ BafA for $25 \mathrm{~min}$ prior to $30 \mathrm{~min}$ incubation with $\left.{ }^{3} \mathrm{H}\right]$ CHLQ for assessment of v-ATPase function. CHLQ accumulation is expressed as difference between basal and BafA-treated for each condition ( $\Delta$ BasalBafA). These data are from the same set of experiments as the data displayed in Figure 5E. Values are displayed as mean \pm SEM. ${ }^{*} p<0.05$; N.S., not significant. 
(A)

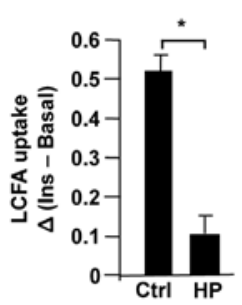

(B)

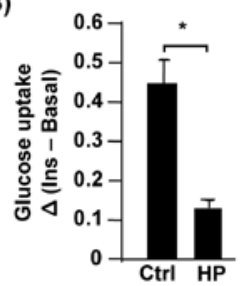

(C)

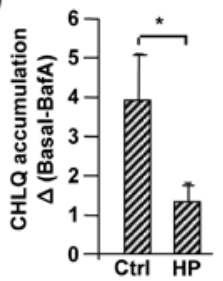

(D)

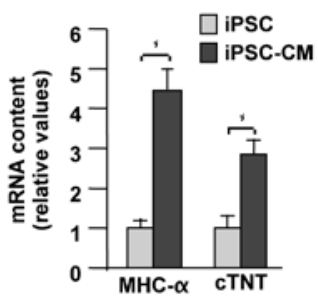

Figure S6: Related to Figure 6

Human iPSC-derived cardiomyocytes (iPSC-CM) were cultured for $16 \mathrm{~h}$ in control (Ctrl) or HP medium medium prior to further treatment or analysis. (A and B) In each condition, iPSC-CM were incubated without/with $100 \mathrm{nM}$ insulin (Ins) for $15 \mathrm{~min}$ prior to execution of $(\mathbf{A})\left[{ }^{14} \mathrm{C}\right]$ palmitate, $(\mathbf{B})$ $\left[{ }^{3} \mathrm{H}\right]$ glucose uptake, or Western blot analysis of phosphorylation of Akt and S6 (see Figure 6C). (A and B) The effect of insulin is expressed as difference between acute insulin-stimulated and basal for each condition ( $\triangle$ Ins-Basal). These data are from the same set of experiments as the data from Figure $6 \mathbf{A}$ and B. (C) In parallel, at each condition, iPSC-CM were treated either without/with $100 \mathrm{nM}$ Bafilomycin-A (BafA) for $25 \mathrm{~min}$ prior to $30 \mathrm{~min}$ incubation with $\left[{ }^{3} \mathrm{H}\right]$ Cloroquine (CHLQ) for assessment of v-ATPase function. CHLQ accumulation is expressed as difference between basal and BafA-treated for each condition ( $\triangle$ Basal-BafA). These data are from the same set of experiments as the data displayed in Figure 6C. (D) mRNA expression of myosin heavy chain (a isoform; MYH6) and cardiac troponin T (TNNT2). Values are displayed as mean \pm SEM. ${ }^{*} \mathrm{p}<0.05$; N.S., not significant.

N.S., not significant. 



\title{
Chapter 4
}

\section{Fluorescent labeling of fatty acid transporter CD36 in the extracellular loop}

\author{
Yilin Liu ${ }^{1}$, Ricardo Rodriguez-Calvo ${ }^{1}$, \\ Xiaoqing $\mathrm{Zhu}^{1}$, Jos Broers ${ }^{2}$, \\ Jan F.C. Glatz ${ }^{1}$, Joost J.F.P. Luiken ${ }^{1}$, \\ Dietbert Neumann ${ }^{1 \#}$
}

Departments of ${ }^{1}$ Molecular Genetics and ${ }^{2}$ Molecular Cell Biology, CARIM School for Cardiovascular Diseases, Maastricht University, P.O. Box 616, 6200 MD Maastricht, the Netherlands

"Correspondence: d.neumann@maastrichtuniversity.nl 


\begin{abstract}
Cardiac lipid uptake is mainly regulated by the sarcolemmal localization of the fatty acid transporter CD36. Upon stimulation of cardiomyocytes by insulin or contraction, CD36 translocates from intracellular storage compartments (endosomes) to the sarcolemma to increase fatty acid uptake. However, sarcolemmal CD36 presence, and therefore fatty acid uptake, also increases upon lipid oversupply, initiating a vicious cycle of cardiac lipid accumulation and lipid-induced insulin resistance, ultimately leading to cardiac dysfunction. Recent evidence indicates the crucial involvement of the vacuolar $\mathrm{H}^{+}$-ATPase (v-ATPase) and specifically the assembly status of its $\mathrm{V}_{0} / \mathrm{V}_{1}$ subunits in the trafficking of CD36. In order to unravel the CD36 trafficking dynamics and investigate v-ATPase co-localization with CD36 in endosomes, we aimed at live cell imaging of CD36 movement using fluorescently labeled CD36. We opted for biarsenical dyes for CD36 protein labeling in the extracellular domain, which required the introduction of a tetracysteine Cys-Cys-Pro-Gly-Cys-Cys sequence. Because insertion of the tetracysteine motif might interfere with CD36 protein function, we selected three different sites for initial analyses. As determined by Western blotting, lentiviral transduction of human myc-DDK-tagged CD36(hCD36) wildtype and mutant proteins yielded the corresponding proteins in mouse HL-1 cardiomyocytes. However, only the tetracysteine mutant 2 (hCD36-TC2) showed a normal biological response in fatty acid uptake assays. Using the biarsenical dye fluorescein arsenical hairpin binder (FlAsH) and fluorescent microscopy, a cytosolic speckled pattern reminiscent of endosomes was observed for hCD36-TC2 under basal culturing conditions. Co-staining of fixed cells using antibodies directed against myc showed co-localization with the FlAsH signal. Furthermore, we found colocalization of the membrane associated (cytoplasmic) $V_{1}$ part of v-ATPase, but only under low palmitate culturing conditions. In contrast, the integral membrane part $\mathrm{V}_{0}$ of v-ATPase co-localized with CD36 in all conditions tested, suggesting that $\mathrm{V}_{1}$ dissociates from $\mathrm{V}_{0}$ in high-palmitate containing media. In summary, hCD36-TC2 is a suitable candidate for the application of biarsenical dyes in future CD36 live imaging studies.
\end{abstract}




\section{Introduction}

Accumulation of fatty acids and their metabolites in cardiomyocytes is increasingly being recognized as a major cause for cardiac insulin resistance, ultimately leading to cardiac dysfunction and together referred to as diabetic cardiomyopathy. CD36, also known as fatty acid translocase (FAT), is the predominant fatty acid transporter in the heart, mediating an estimated $70 \%$ of the cardiac fatty acid flux [1]. Under normal conditions, CD36 cycles between intracellular storage compartments and the sarcolemma. Hormonal or mechanical stimuli induce translocation of CD36 to the sarcolemma, and hence enhance fatty acid uptake and subsequent metabolism. This demonstrates that CD36 translocation provides a platform for dynamic adaptation of the fatty acid transport rate into cardiomyocytes in response to physiological stimuli [2, 3]. Studies in insulin-resistant rodent models have shown CD36 abundance at the cell surface to be markedly increased due to a sustained relocation from endosomes in the absence of changes in CD36 total expression, indicating that fatty acid oversupply induces alterations in subcellular CD36 cycling $[4,5]$. The aberrant high sarcolemmal CD36 presence causes a chronically elevated influx of fatty acids into cardiomyocytes, which is directly coupled to lipid accumulation, insulin resistance and contractile dysfunction. Thus, permanent CD36 relocation plays a vital role in the development of diabetic cardiomyopathy [4].

Evidently, permanent CD36 relocation to the sarcolemma is caused by malfunctioning of the physiological process whereby CD36 recycles between the endosomal compartment and the sarcolemma. Nevertheless, little is known about the cellular mechanisms involved in CD36 translocation. Previous studies have identified vacuolar-type $\mathrm{H}^{+}$ATPase (v-ATPase) as being involved in this process [6]. As a multi-unit proton pump occurring in acidic organelles, v-ATPase is responsible for endosomal acidification. v-ATPase consist of two sub-complexes, i.e., integral membrane sub-complex $V_{0}$ and cytoplasmic sub-complex $V_{1}$ [7]. Biochemical assays have demonstrated that pharmacologically induced v-ATPase inhibition (using the potent inhibitor bafilomycin-A) leads to expulsion of CD36 from the endosomes to the sarcolemma, indicating an important role of v-ATPase in the regulation of CD36 trafficking [6]. Our recent study further suggested that during lipid overload CD36 translocation to the sarcolemma is due to the release of the $\mathrm{V}_{1}$ sub-complex into the cytoplasm, resulting in a loss of v-ATPase proton pumping activity and hence a decreased acidification of the endosomes. These novel findings indicate that v-ATPase acts as a lipid sensor, which upon disassembly, sets the heart on a road to insulin resistance and contractile dysfunction (Chapter 3). 
Live cellular imaging is an effective approach to visualize the CD36 translocation process. Green fluorescent proteins (GFP) have been widely used for biological imaging to investigate protein localization, intracellular trafficking, and protein-protein interactions [8]. Previous studies have shown both immunofluorescently-labeled and GFP-tagged CD36 trafficking in either fixed or live Chinese hamster ovary (CHO) cells $[9,10]$. Nevertheless, both GFP and antibodies are relatively large proteins, which may interfere with the function or alter the trafficking process of a target protein. Furthermore, the use of GFP as an independent protein domain is restricted to the $\mathrm{N}$ - or $\mathrm{C}$ - terminus of the target protein and has the disadvantage of having fluorescent signals through the entire translocation process, limiting extracellular applications.

Efforts to reduce the considerable size of labels and preserve the native biological function of proteins were undertaken over the past few years, and one of the most successful approaches is the genetically encoded tetracysteine technology, which involves live cell protein staining $[11,12]$. This approach is based on the binding of the tetracysteine sequence Cys-Cys-Xaa-Xaa-Cys-Cys (with Xaa as a changeable residue), in which the Pro-Gly dipeptide is preferentially incorporated because of its high affinity for biarsenic dyes, like fluorescein arsenical hairpin binder (FlAsH) and resorufin arsenical hairpin binder (ReAsH) $[13,14]$. FlAsH or ReAsH are used combined with ethane dithiol (EDT) as a nonfluorescent complex. When either FlAsH-EDT 2 or ReAsH-EDT 2 binds to the tetracysteine sequence, EDT is displaced whereafter the FlAsH or ReAsH becomes highly fluorescent in green or red, respectively. As a possible alternative way of protein labeling, this approach provides several advantages. Since the six-amino acid tag is exceptionally small, it can be easily inserted at different sites in the target protein and is expected to minimize interference with the target protein's functioning.

In our current study, we selected three different positions in the extracellular loop of human CD36 to incorporate the tetracysteine motif as potential FlAsH labelling site. Upon lentiviral transduction we used Western blotting, functional assays and fluorescent microscopy analyses to identify a tetracysteine construct that would not interfere with CD36 functioning. We further investigated the co-localization of v-ATPase $V_{0} / V_{1}$ subunits with tetracysteine-tagged CD36, which is expected to be dynamic based on our previous studies.

\section{Methods}

\section{Plasmid Constructs}

The tetracysteine motif Cys-Cys-Pro-Gly-Cys-Cys was engineered into the extracellular loop of CD36 by two-step PCR. Three different sites were selected for this insertion, encoded at the following amino acids (aa) positions: hCD36-TC1: aa48; hCD36-TC2: aa89; hCD36-TC3: aa398 (Figure 1). The primers spanning indicated 


\section{Sense}

General primer $5^{\prime}$ - AGGAGATCTGCCGCCGCGATCGCC - 3' (BgIII)

hCD36-TC1

hCD36-TC2

hCD36-TC3
5' - TTAAGCAAAGAGTGCTGTCCAGGATGCT GCGTCCTTATACGT - 3'

5' - CGAAGAAGGTACTGCTGTCCAGGATGCT GCAATTGCTTTTAA - 3'

\section{5' - GCCATCAGAAAATGCTGTCCAGGATGCT GCAATTCAAGTATT - 3'}

\section{Antisense}

5'- CCACCCGGGATCTGTTCAGGAAACAGCT ATG - 3' (Smal)

5 - TTAAAAGCAATTGCAGCATCCTGGACAG CAGTACCTTCTTCG - 3'

5' - ACGTATAAGGACGCAGCATCCTGGACAG CACTCTTTGCTTAA - 3'

5' - AATACTTGAATTGCAGCATCCTGGACAG CATTTTCTGATGGC - 3'

regions in cDNA of myc-DDK-tagged hCD36 were as follows:

Mutants were confirmed by sequencing (Eurogentec, Maastricht, The Netherlands). The three mutant sequences of CD36 were cut from pCMV6 construct (Origene, Rockville, United States) using the restriction enzymes, BglII/SmaI, and subcloned to the lentiviral vector $\mathrm{pLVX}$ by ligation to the same restriction sites.

\section{Virus Production and Infections}

HEK293T cells were plated at $40 \%$ confluency in $10 \mathrm{~cm}$ dishes. After $24 \mathrm{~h}$, lentiviral vector pLVX-Puro (Clontech, No. 632164), together with the two packaging plasmids, PAX2 and pMD2.G (Envelope and GagPol), were transfected into 293T cells by using P-PEI. The medium supplemented with $10 \%$ fetal calf serum (FCS) and $1 \% 100 \mathrm{U} / \mathrm{ml}$ penicillin-streptomycin was refreshed, $24 \mathrm{~h}$ after transfection. The medium was harvested after both $48 \mathrm{~h}$ and $72 \mathrm{~h}$, filtered through $0.45 \mu \mathrm{m}$ filters and used for infection. HL-1 cardiomyocytes were cultured in Claycomb medium at $60 \%$ confluency in 6-well plate as previously described [15]. After seeding for $3 \mathrm{~h}$, cell culture medium was aspirated and the cells were infected with viral supernatant in the presence of polybrene $(8 \mu \mathrm{g} / \mathrm{ml})$ overnight. After recovery for $48 \mathrm{~h}$ with fresh medium, cells were selected with puromycin $(4 \mu \mathrm{g} / \mathrm{ml})$ for $48 \mathrm{~h}$. Thereafter cells were used for experiments as described.

\section{Western Blotting}

The lentivirally infected cell lysates were prepared and analyzed by Western blotting as previously described [16]. The following primary antibodies were used: anti-hCD36 (1: 20000 in TBST; kindly provided by Dr. Tandon, Bethesda, USA) and anti-DDK (1: 2000 in 5\% BSA in TBST; Origene). Primary antibodies were detected by anti-mouse secondary antibody (1:20000 in TBST; Dako). 


\section{$\left[{ }^{14} \mathrm{C}\right]$ Palmitate Uptake Assays}

The infected different HL-1 cardiomyocytes were cultured on pre-coated coverslips in 12-well plate. Prior to uptake assay, cells were incubated in serum-free depletion medium for $16 \mathrm{~h}$. Thereafter, cells were treated either with or without $200 \mathrm{nM}$ insulin for 30 min. Subsequently, $\left[1-{ }^{14} \mathrm{C}\right]$ palmitate (coupled to BSA in a palmitate/BSA ratio of 1:3) was added to final concentrations of $20 \mu \mathrm{M}$. After $10 \mathrm{~min}$, uptake was terminated by Stop medium (Serum-free depletion medium, $0.2 \mathrm{mM}$ phloretin). After transfer of the coverslips into new cell culture wells with stop medium, cells were lysed by $1 \mathrm{M} \mathrm{NaOH}$, and then cell-associated palmitate was measured by scintillation counting of ${ }^{14} \mathrm{C}$.

\section{CD36 Labeling with FlAsH-EDT $T_{2}$ and Immunofluorescence}

HL-1 cardiomyocytes infected with the various CD36 constructs were cultured on gelatin/fibronectin-coated coverslips in 24-well plate. Prior to labeling, cells were incubated in serum-free depletion medium for $16 \mathrm{~h}$. Next day, medium was removed and cells were washed twice with HBSS with $5.6 \mathrm{mM}$ glucose. Cells were incubated with 500 $\mathrm{nM}$ FlAsH-EDT 2 (Thermofisher) in HBSS/glucose medium for $1 \mathrm{~h}$ at $37^{\circ} \mathrm{C}$. After the aspiration of FlAsH-EDT ${ }_{2}$, nonspecifically bound dye was removed by incubation with $250 \mu \mathrm{M}$ EDT for $10 \mathrm{~min}$ at $37^{\circ} \mathrm{C}$. Cells were washed seven times with HBSS/glucose and fixed with $4 \%$ formaldehyde afterwards for $10 \mathrm{~min}$ at room temperature. Subsequently, cells were rinsed twice in PBS and permeabilized by $0.2 \%$ Triton X-100 in PBS for 15 min at room temperature, followed by 90 min of incubation with anti-Myc antibody (Cell Signaling Technology; 1:4000), anti-v-ATPase a2 (Abcam; 1:100), or anti-v-ATPase B2 (Abcam; 1:1000) in blocking buffer (5\% FCS in 0.02\% Triton X-PBS) and Texas Red conjugated goat anti-mouse or rabbit antibody (SouthernBiotech; 1:100) in blocking buffer for $1 \mathrm{~h}$ at room temperature in darkness. Coverslips were washed and mounted with DABCO-glycerol medium (Sigma-Aldrich) containing DAPI (1:10,000; SigmaAldrich).

\section{Confocal imaging}

Fixed cells were imaged by confocal microscopy as previously described [17]. Images were analyzed by ImageJ software. Brightness and contrast of images was adjusted to the same settings where needed.

\section{Statistics}

All data are presented as means \pm SEM. Statistical analysis was performed by using two-sided Student's $t$-test. P-values of less than 0.05 were considered statistically significant. 


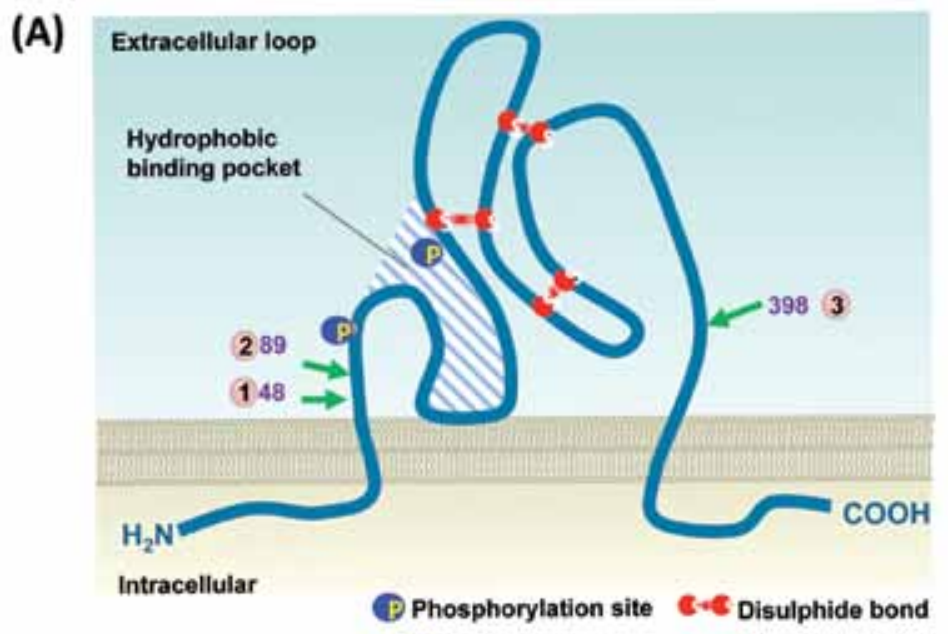

(B) hCD36-TC1 GAAGGT ACIAATT GCT
hCD36-TC2 CAAAGA GIGT CCT TAT
hCD36-TC3 TCA GAA AAIAATT CAA

Figure 1. Schematic presentation of CD36 with target sites for insertion of the tetracysteine motif. (A) The proposed topology of CD36 with different numbers of potential sites, indicating the amino acid residue locations on the extracellular loop where the tetracysteine motif was inserted. (B) Part of the CD36 sequences, showing the exact positions of the potential insertion sites. Scheme adapted from reference 1 .

\section{Results}

Visualizing proteins with the biarsenical reagents FlAsH-EDT 2 is based on the binding to a short peptide sequence of the general structure Cys-Cys-Xaa-Xaa-Cys-Cys, preferably flanking a Pro-Gly dipeptide [14]. We predicted three different sites that were remote from either the hydrophobic binding pocket or the two phosphorylation sites on the extracellular loop, i.e., at amino acid positions 48, 89, and 398, respectively (Figure 1). To genetically encode the tetracysteine motif, Cys-Cys-Pro-Gly-Cys-Cys (CCPGCC), we designed different primers containing the sequence 5'-XXXXXTGCTGTCCAGGATGCTGCXXXXX-3' and general primers for the sequence of CD36, which was incorporated into cDNA of myc-DDK-tagged hCD36 by two steps of PCR and subcloned to lentivirus vector, PLVX (Figure 2).

In order to establish stable CD36 expressing cell lines, HL-1 mouse cardiomyocytes were infected with lentiviruses carrying empty vector, wildtype or mutant hCD36, the latter carrying an insertion of the tetracysteine motif at one of the three different sites within the extracellular domain. The cells infected with empty lentiviral vector or wildtype hCD36 were taken as controls. To verify hCD36 expression, we used CD36 antibodies that specifically recognize the human but not the mouse protein. In addition, 
Step 1: Two PCR reactions in parallel using mutant primer carrying tetracysteine motif

PCR 1 Forward primer PCR 2 Reverse primer
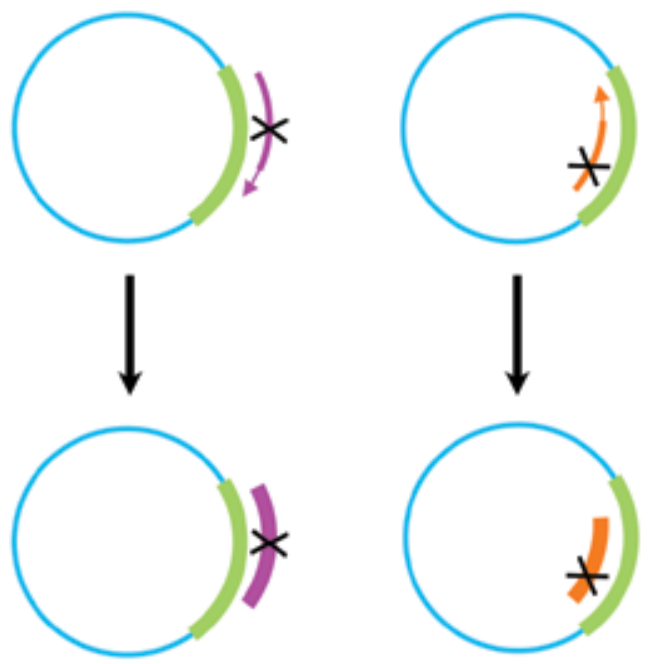

Step 2: Combining the two PCR products from step 1
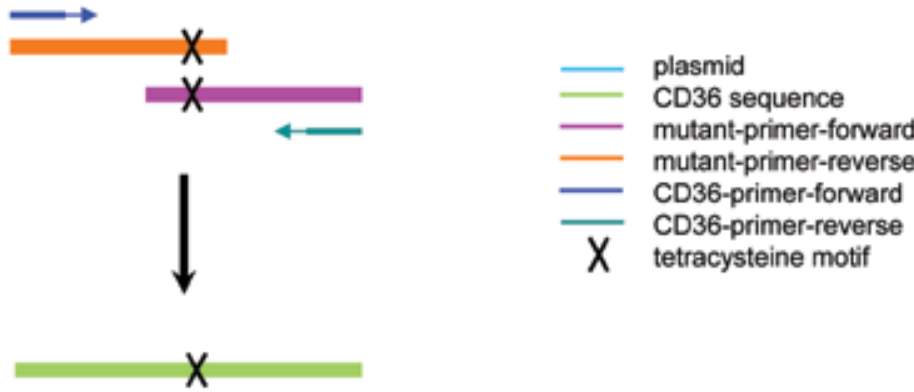

Figure 2. Two steps of PCR to generate different CD36 mutants. Two PCR reactions were performed in parallel by using different mutant primers, which carried the tetracysteine motif at different positions. Thereafter, the products from the first step were applied as templates, and the primers for the amino acid sequence of CD36 were used to generate different CD36 mutants.

we exploited the vector encoded DDK-tag. Accordingly, cell lysates were analyzed by Western blotting, revealing that neither hCD36 nor DDK-tag was detected in control empty vector, whereas hCD36 wildtype and mutants were detected using both antibodies (Figure 3). These results suggested that the corresponding proteins were being appropriately expressed in HL-1 cardiomyocytes. To investigate whether the different mutants still preserve fatty acid transport function compared to transfected empty 


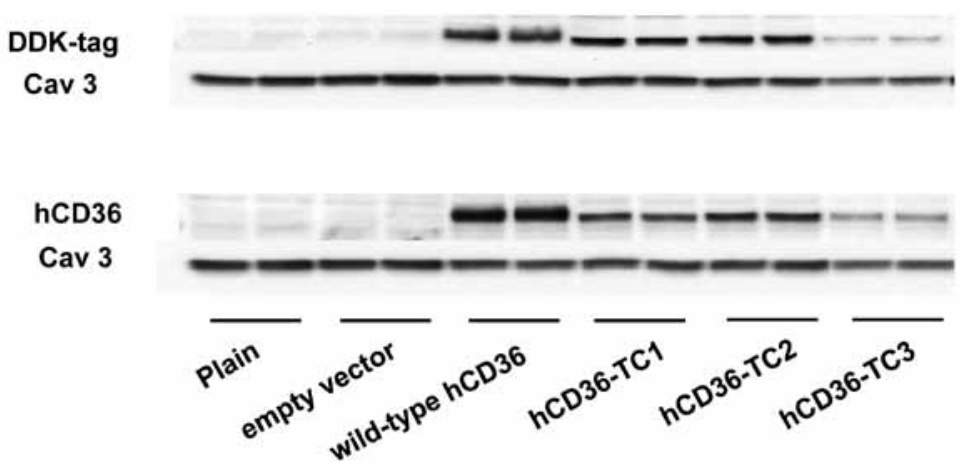

Figure 3. CD36 expression in different HL-1 cell lines after lentiviral transduction. Cells were not infected (plain), or infected with empty vector, wild-type hCD36, or the indicated CD36 constructs. After 48 hours of culturing, cell lysates were prepared for Western blot analyses. Overexpression of hCD36 and its DDK-tag were detected using specific antibodies and caveolin-3 as loading controls. Representative blots $(n=3)$ are shown.

vector, the various stable cell lines were treated with serum-free depletion medium for 16 h. We then assessed long-chain fatty acid (LCFA) uptake in the absence or presence of insulin. Basal LCFA uptake was increased 1.2-1.4-fold in both the wildtype hCD36 and two tetracysteine-tagged hCD36 transfected cells (hCD36-TC2 and hCD36TC3) compared to empty vector (Figure 4, white bars), indicating increased LCFA uptake capacity due to the additional (human) CD36 on top of the endogenous (mouse) CD36. Short-term insulin stimulation enhanced LCFA uptake by 1.2-fold in non-transfected HL-1 cells. Similarly, the insulin effect was also observed in control empty vector (1.2fold), wildtype hCD36 (1.1-fold), as well as hCD36-TC2 (1.2-fold), but not in hCD36TC1and -TC3 (Figure 4, solid bars). Taken together, based on the enhancement of LCFA uptake and the retaining of insulin stimulation, the hCD36-TC2 mutant was selected for further studies.

We next examined the cellular fluorescent labeling of tetracysteine-tagged hCD36 with FlAsH-EDT 2 in combination with immunofluorescence by an anti-myc antibody. In cells with the control empty vector, immunofluorescence or FlAsH-EDT 2 did not result in any fluorescent signals. Immunofluorescence exhibited a markedly speckled pattern in both wild-type hCD36 and hCD36-TC2, as shown by myc-antibody. A similar speckled pattern from FlAsH-EDT 2 was seen in hCD36-TC2, not in wild-type hCD36, likely reflecting the binding of tetracysteine motif and FlAsH (Figure 5, upper panels). The speckled pattern is in line with an endosomal localization. Notably, the speckled pattern of hCD36-TC2 from FlAsH-EDT 2 co-localized with myc, indicating that the speckles indeed represented hCD36 presence in endosomes (Figure 5, lower panels).

We further assessed FlAsH-EDT 2 staining in both wildtype hCD36 and hCD36-TC2 upon different stimulations. FlAsH-EDT 2 staining under basal conditions yielded an in- 


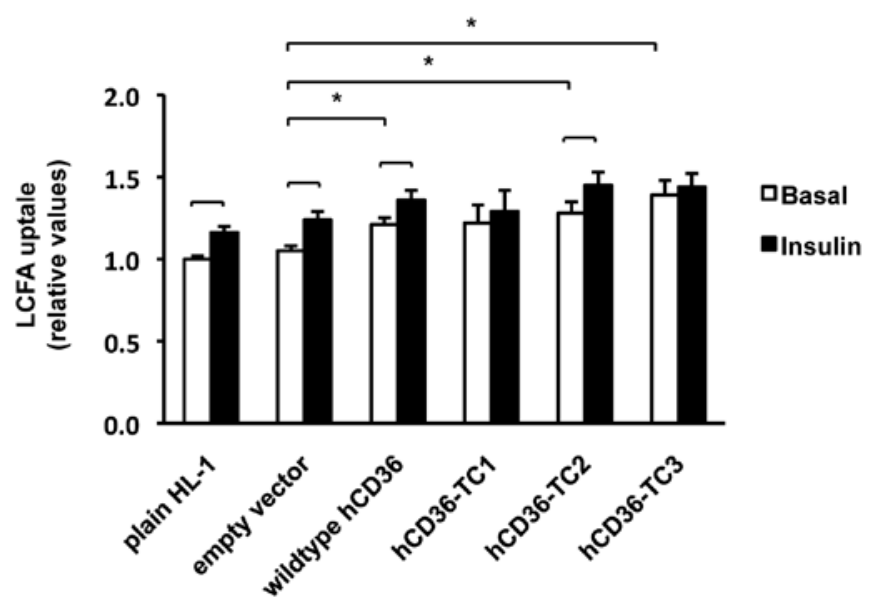

Figure 4. Long-chain fatty acid uptake into differently transfected HL-1 cell lines under basal and short-term insulin stimulation conditions. Prior to uptake assay, different cell lines were incubated in serum-free depletion medium for $16 \mathrm{~h}$. Thereafter, cells were treated either with or without $200 \mathrm{nM}$ insulin for $30 \mathrm{~min}$ and ${ }^{14}[\mathrm{C}]$ palmitate uptake were measured, subsequently. Values are displayed as mean \pm SEM. $(n=3) * p<0.05$; N.S., not significant.

tracellular speckled pattern. This staining largely disappeared upon both short-term insulin stimulation and bafilomycin-A treatment (Figure 6A). These observations may indicate that in both conditions CD36 translocates to the cell surface. Due to technical problems, however, we are currently unable to detect CD36 at the cell surface (see Discussion). Additionally, long-term high palmitate treatment led to a loss of the speckled pattern. When this condition was subjected to short-term insulin or bafilomycin-A stimulation, no further loss of the speckled staining was observed (Figure 6A). Quantification of the staining pattern showed long-term high-palmitate treatment induced a decrease to $50 \%$ in endosomal staining compared to basal condition, which also occurred upon short-term stimulations with insulin or bafilomycin-A. No further decrease was seen upon insulin or bafilomycin-A treatment after long-term high-palmitate treatment (Figure 6B). Thus, wild-type hCD36 and hCD36-TC2 showed similar biological responses in all tested conditions. Therefore, hCD36-TC2 appears to be a suitable construct for further live cell imaging studies.

Previously, we found that lipid-induced CD36 relocation to the cell surface was due to disassembly of the two sub-complexes $\mathrm{V}_{0}$ and $\mathrm{V}_{1}$ of $\mathrm{v}$-ATPase and the resulting loss of v-ATPase activity (Chapter 3). This conclusion was based on both immunoprecipitation and subcellular fractionation experiments. In order to visualize lipid-induced CD36 relocation and v-ATPase disassembly by microscopy, we combined FlAsH-EDT 2 staining with immunofluorescence using antibodies directed against either v-ATPase a2 (part of the integral membrane $\mathrm{V}_{0}$ sub-complex) or v-ATPase $\mathrm{B} 2$ (part of the membrane- 


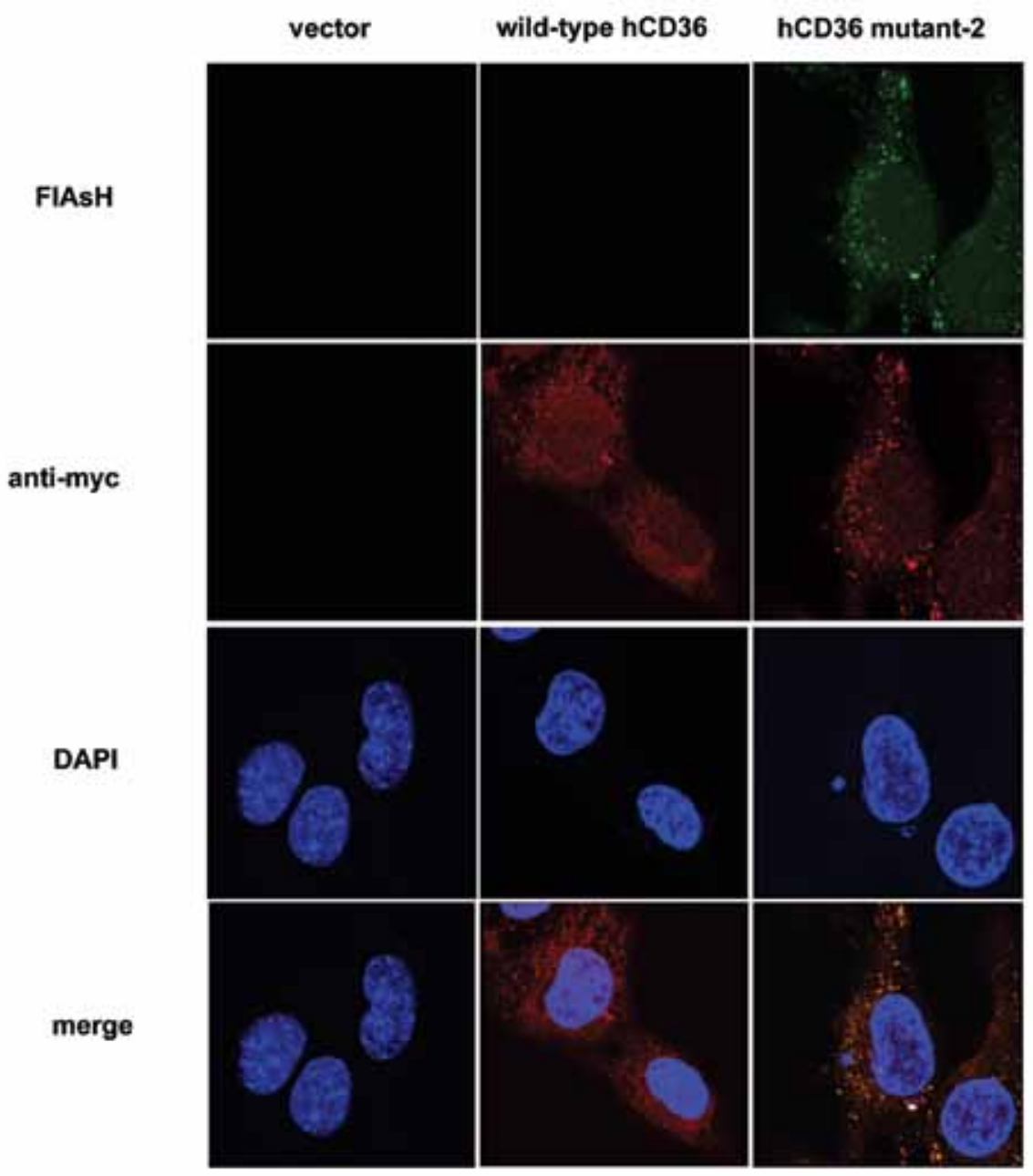

Figure 5. Labelling of tetracysteine-tagged hCD36 and immunofluorescence with anti-myc antibody. Shown are plain HL-1, cells stably overexpressing wildtype of $h C D 36$, and $h C D 36-T C 2$, as indicated. Cells were fixed and stained with FIAsH-EDT $($ in green) and thereafter with an anti-myc antibody that was detected by an Texas red-labeled secondary antibody (in red) and with DAPI for nuclei (in blue), after which co-localization was assessed by confocal imaging. Data are representative of 3 experiments. Data have been adjusted for brightness and contrast to obtain best quality of fluorescent images.

associated $\mathrm{V}_{1}$ sub-complex). a2 subunit staining presented as a speckled pattern, colocalizing with FlAsH-EDT 2 staining in the basal condition. In contrast, the FlAsH$\mathrm{EDT}_{2}$ staining decreased by $60 \%$ upon high-palmitate treatment. Yet, the a2 speckled pattern remained unchanged upon high palmitate treatment compared to the basal condition (Figure 7). While in the basal condition also the B2 subunit staining was found to co-localize with FlAsH-EDT 2 staining, upon high-palmitate treatment the number of cytosolic speckles decreased by $50 \%$ for B2, similarly as with FlAsH-EDT 2 
(A)

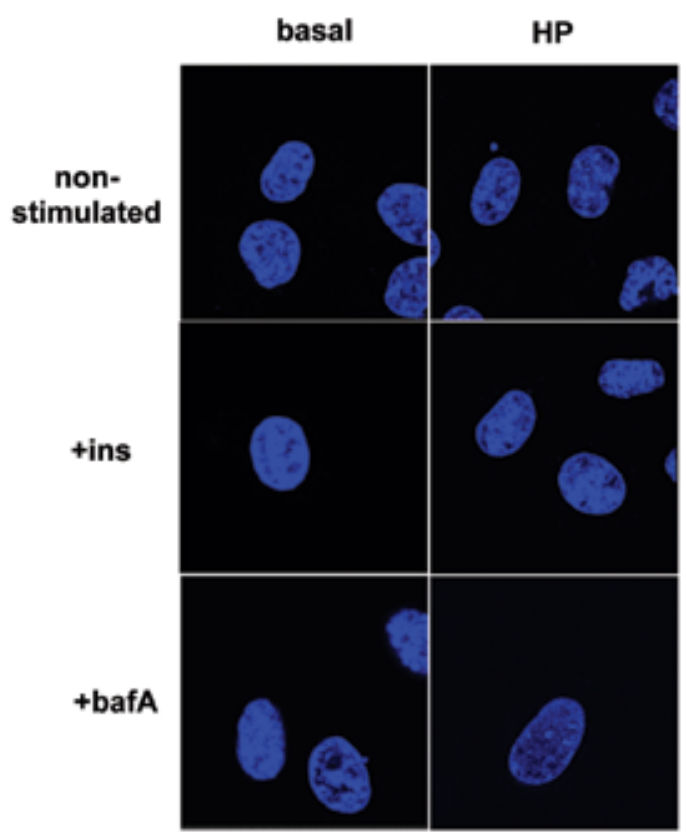

mutant-2hCD36

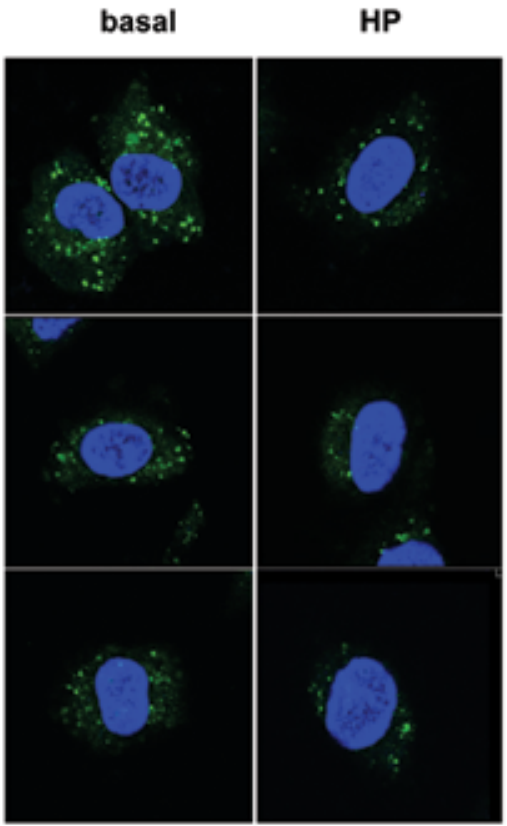

(B)

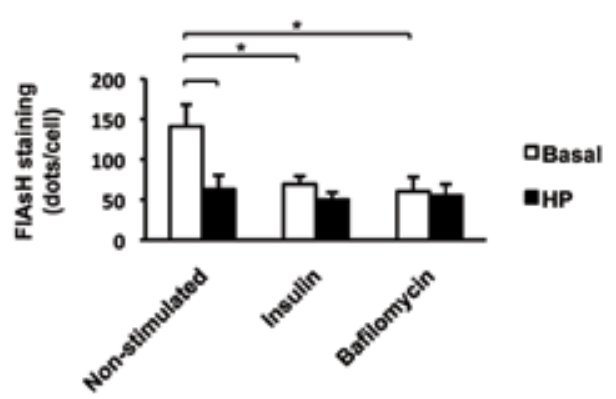

Figure 6. Labelling of tetracysteine-tagged hCD36 upon high-palmitate treatment. Shown are HL-1 cells stably overexpressing wild-type hCD36 or hCD36-TC2, as indicated. Cells were incubated with serum-starved medium or high-palmitate (HP) medium for $16 \mathrm{~h}$ and thereafter were treated with $30 \mathrm{~min}$ of $200 \mathrm{nM}$ insulin or $100 \mathrm{nM}$ bafilomycin-A. (A) Cells were fixed and stained with FIAsH-EDT 2 (in green). Data have been adjusted for brightness and contrast to obtain best quality of fluorescent images and are representative of 3 experiments. (B) Quantification of FIAsH-EDT 2 staining in hCD36-TC2 with non-stimulated, insulin, and bafilomycin-A stimulation. Values are displayed as mean \pm SEM. $(n=3){ }^{*} p<0.05$; N.S., not significant.

(Figure 7) These results reconfirm the translocation of CD36 upon exposure to media with high lipid concentrations. Further, the data suggest the dissociation of v-ATPase $V_{1}$ from the endosomal membrane bound $\mathrm{V}_{0}$ sub-complex by high-palmitate. In summary, lipid-induced CD36 relocation and v-ATPase disassembly was observed at the 
microscopic level.

\section{Discussion}

Tetracysteine-based labelling of protein has been successfully achieved via biarsenical dyes, such as FlAsH [11-13]. As the tetracysteine sequence, Cys-Cys-Pro-Gly-Cys-Cys, rarely appears in endogenous proteins [11], incorporating the sequence into target proteins generates a small but highly specific target for FlAsH protein labeling. In our current study, we introduced tetracysteine motifs to the extracellular domain of hCD36 at three different positions. If transduced with hCD36-TC2 (carrying the insert at amino acid postition 89), HL-1 cardiomyocytes, after selection with antibiotics, showed overexpression of hCD36 in conjunction with enhanced fatty acid uptake and sensitivity to different stimuli, i.e., similar to the wildtype construct, whereas the other two mutants did not fulfil all the criteria. We also provided evidence that FlAsH-EDT 2 labeling combined with immunofluorescence staining the myc epitope encoded by hCD36-TC2 exhibited co-localization, suggesting that the speckled pattern resulting from FlAsHstaining indeed represents CD36. The particulate pattern is furthermore in agreement with the expected endosomal localization of CD36. Moreover, we demonstrated that different stimuli (insulin, bafilomycin A, and palmitate) triggered the disappearance of hCD36-TC2 from the endosomal compartment in keeping with the known translocation of CD36 to the cell surface. However, we could not see the increased surface localization of CD36, which may be due to the relatively weak staining in combination with the thin membrane. Nevertheless, our findings suggest hCD36-TC2 as a suitable candidate to label with biarsenical dyes for live cell imaging studies.

The major advantage of the small size of a tetracysteine motif insertion is that this methodology to label proteins can be utilized for many applications. Despite the obvious merits of tetracysteine-based FlAsH-EDT 2 staining, there are also disadvantages that may restrict the use of FlAsH-EDT ${ }_{2}$ and thus may affect the application of this approach. Firstly, low expression of the target protein may not produce a good signal and in the meanwhile give much noise. Therefore, appreciable overexpression of the target protein is required for better labeling. Secondly, the non-specific binding with excess dyes gives a relatively high background, which needs to be removed with high-concentrations of EDT, followed by multiple washing steps with HBSS/glucose compared to the standard protocol [18]. Perhaps, the requirement of washing steps is also cell-dependent, which has to be tested experimentally. Thirdly, to study selectively the cell surface-localized CD36, membrane-impermeable biarsenical dyes are required. Yet, the commercially available FlAsH-EDT 2 and $\mathrm{ReAsH}_{2} \mathrm{EDT}_{2}$ each are membrane-permeable, and thus are expected to have access to the entire cellular CD36 population. A sulfonated derivative of FlAsH (sFlAsH), which has been described to be membrane-impermeable, is not 


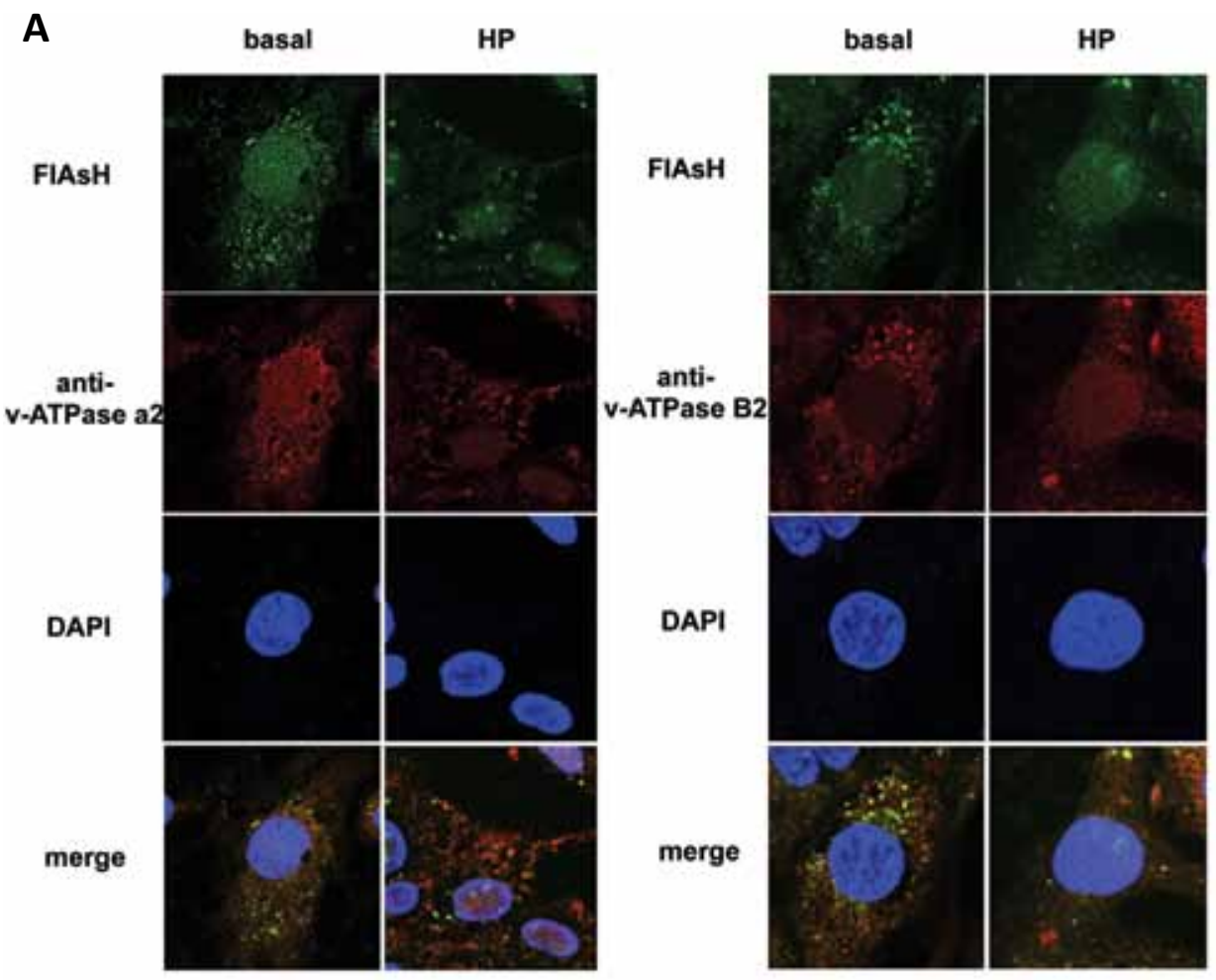

B
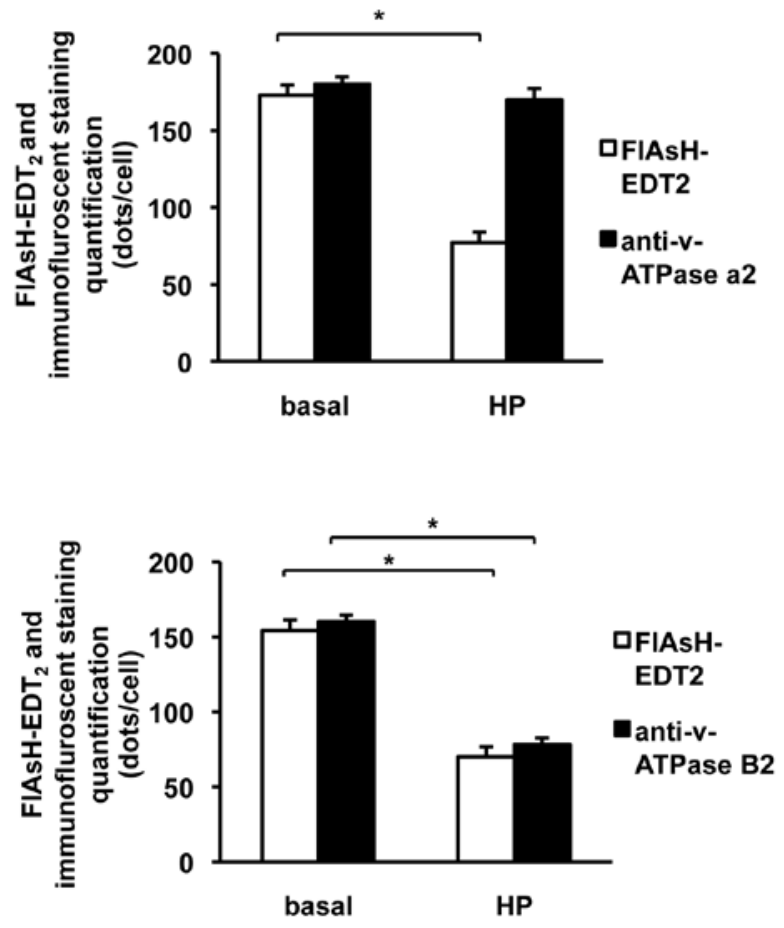
Figure 7. Labelling of tetracysteine-tagged hCD36 and immunofluorescence with anti-vATPase a2 or B2 antibodies. (A) Shown are HL-1 cells that are stably overexpressing hCD36-TC2 upon basal or high-palmitate (HP) treatment, as indicated. Cells were fixed and stained with FIAsH$\mathrm{EDT}_{2}$ (in green) and thereafter with anti-v-ATPase a2 or B2 antibodies that was detected by an Texas red-labeled secondary antibody (in red) and with DAPI for nuclei (in blue), after which co-localization was assessed by confocal imaging. Data are representative of 3 experiments. (B) Quantification of FIAsH-EDT 2 staining and immunofluorscence with v-ATPase a2 or B2. Data have been adjusted for brightness and contrast to obtain best quality of fluorescent images.

commercially available, and the synthesis of such compound is beyond our expertise [14]. An easier method to exclusively label extracellular tetracysteines is to pre-incubate FlAsH-EDT 2 with DMPS (dimercaptopranesulfonate sodium salt, Sigma-Aldrich). This compound exchanges the membrane-permeable EDT for the charged DMPS and has been applied successfully in tetracysteine-tagged proteins in intact cells [18]. Following this protocol, we made several attempts to exclusively label surface CD36. However, this procedure proved only partially effective, because despite a better surface CD36 labeling, some intracellular CD36 staining remained (data not shown). For future studies, a further optimization of the cell surface CD36 staining protocol is required, or further expertise is needed to synthesize sFlAsH.

Another technology similar to FlAsH-EDT 2 uses small fluorogen activating peptide (FAP), a novel fluorescent biosensor that can be turned on and off by adding or removing fluorogen, or by changing the signal wavelength through substitution of one fluorogen for another $[19,20]$. This technology is particularly useful in trafficking studies since cell surfacelabeling is feasible by incubating mutantFAP-protein-transfected cells with a membrane impermeable fluorogen. However, the relative large size of the FAP-tag (25 KDa) limits its fusion site to the protein N-terminus, and therefore is not suitable for CD36 trafficking studies, as both $\mathrm{C}$ - and N-terminals are localized intracellularly and would thereby also inhibit the binding of FAP-tag to impermeable fluorogen. The combination of those two techniques, offering a relative small size of the tag and an impermeable fluorogen, we would judge as the ideal approach for CD36 trafficking studies. However, such technology is unavailable as yet. Currently, novel biarsenical dyes with improved properties are in development, and hopefully the expected progress will make this technique more applicable in the future.

We previously identified v-ATPase as a mediator of lipid-induced CD36 relocation to the sarcolemma and the resulting lipid accumulation and development of cardiac insulin resistance (Chapter 3). Assembly/disassembly of the two v-ATPase sub-complexes, $\mathrm{V}_{1}$ and $\mathrm{V}_{0}$, is the main mechanism of regulation of $\mathrm{v}$-ATPase activity in yeast [21]. This mechanism is sensitive to glucose concentrations, which favor assembly and, hence, $\mathrm{v}$-ATPase activation [22]. Also in the mammalian heart, v-ATPase appears to be regulated by assembly/disassembly, with high lipid concentrations inducing the migration of the 
$\mathrm{V}_{1}$ sub-complex into the cytoplasm, whereas the $\mathrm{V}_{0}$ sub-complex remained integral to the endosomal membrane (Chapter 3). This novel mechanism was revealed using both immunoprecipitation and subcellular fractionation methods (Chapter 3). In the present study, we further investigated lipid-induced v-ATPase disassembly in cardiomyocytes by direct microscopic observation. Indeed, the combination of $\mathrm{FlAsH}_{\mathrm{H}} \mathrm{EDT}_{2}$ staining and immunofluorescence by antibodies against subunits from the two different v-ATPase sub-complexes further underscores that lipids cause the migration of $\mathrm{V}_{1}$ into the cytoplasm followed by increased CD36 translocation (Figure 7). Taken together, both biochemical and microscopic approaches suggest assembly/disassembly as the primary mechanism of lipid-induced CD36 translocation.

In previous studies, we have successfully applied the fluorescent $\mathrm{pH}$ indicator lysosensor DND-189 to visualize endosomal $\mathrm{pH}$ changes in both primary and HL-1 cardiomyocytes upon lipid oversupply (Chapter 3). Combining biarsenical dyes with lysosensor (in order to stain CD36-containing vesicles and monitor their $\mathrm{pH}$ ) may be a suitable approach to visualize CD36 dynamics in conjunction with v-ATPase function in live cells, for example, upon lipid overexposure. This approach requires the microscopy to be performed at ML-II level, which is not currently possible at our institution. Therefore, we investigated $\mathrm{V}_{0} / \mathrm{V}_{1}$ disassembly in fixed lipid-overloaded cardiomyocytes. In future studies, it will be especially interesting to shed a light on v-ATPase assembly status in human tissue samples, where neither FlAsH-EDT 2 nor lysosensor can be applied. In human heart biopsies obtained from valvular surgery, the difference of colocalization of $\mathrm{V}_{1}$ and $\mathrm{V}_{0}$ with CD36 between control patients and diabetic patients will be interesting to study. Such data may confirm the v-ATPase disassembly in human heart in diabetic cardiomyopathy. 


\section{References}

1. J.F. Glatz, J.J. Luiken, A. Bonen, Membrane fatty acid transporters as regulators of lipid metabolism: implications for metabolic disease, Physiol Rev, 90 (2010) 367-417.

2. J.J. Luiken, D.P. Koonen, J. Willems, A. Zorzano, C. Becker, Y. Fischer, N.N. Tandon, G.J. Van Der Vusse, A. Bonen, J.F. Glatz, Insulin stimulates long-chain fatty acid utilization by rat cardiac myocytes through cellular redistribution of FAT/CD36, Diabetes, 51 (2002) 3113-3119.

3. J.J. Luiken, S.L. Coort, J. Willems, W.A. Coumans, A. Bonen, G.J. van der Vusse, J.F. Glatz, Contraction-induced fatty acid translocase/CD36 translocation in rat cardiac myocytes is mediated through AMP-activated protein kinase signaling, Diabetes, 52 (2003) 1627-1634.

4. D.M. Ouwens, M. Diamant, M. Fodor, D.D. Habets, M.M. Pelsers, M. El Hasnaoui, Z.C. Dang, C.E. van den Brom, R. Vlasblom, A. Rietdijk, C. Boer, S.L. Coort, J.F. Glatz, J.J. Luiken, Cardiac contractile dysfunction in insulin-resistant rats fed a high-fat diet is associated with elevated CD36-mediated fatty acid uptake and esterification, Diabetologia, 50 (2007) 1938-1948.

5. S.L. Coort, D.M. Hasselbaink, D.P. Koonen, J. Willems, W.A. Coumans, A. Chabowski, G.J. van der Vusse, A. Bonen, J.F. Glatz, J.J. Luiken, Enhanced sarcolemmal FAT/CD36 content and triacylglycerol storage in cardiac myocytes from obese zucker rats, Diabetes, 53 (2004) 1655-1663.

6. L.K. Steinbusch, W. Wijnen, R.W. Schwenk, W.A. Coumans, N.T. Hoebers, D.M. Ouwens, W.A. Coumans, N.T. Hoebers, M. Diamant, A. Bonen, J.F. Glatz, J.J. Luiken, Differential regulation of cardiac glucose and fatty acid uptake by endosomal $\mathrm{pH}$ and actin filaments, Am J Physiol Cell Physiol, 298 (2010) C1549-1559.

7. M. Forgac, Vacuolar ATPases: rotary proton pumps in physiology and pathophysiology, Nat Rev Mol Cell Biol, 8 (2007) 917-929.

8. T. Misteli, D.L. Spector, Applications of the green fluorescent protein in cell biology and biotechnology, Nat Biotechnol, 15 (1997) 961-964.

9. Y. Zeng, N. Tao, K.N. Chung, J.E. Heuser, D.M. Lublin, Endocytosis of oxidized low density lipoprotein through scavenger receptor CD36 utilizes a lipid raft pathway that does not require caveolin-1, $J$ Biol Chem, 278 (2003) 45931-45936.

10. J. Zhang, I. Crandall, Expression of both N- and Cterminal GFP tagged huCD36 and their discrepancy in OxLDL and pRBC binding on CHO cells, Lipids Health Dis, 6 (2007) 24.

11. B.A. Griffin, S.R. Adams, R.Y. Tsien, Specific covalent labeling of recombinant protein molecules inside live cells, Science, 281 (1998) 269-272.
12. G. Gaietta, T.J. Deerinck, S.R. Adams, J. Bouwer, O. Tour, D.W. Laird, G.E. Sosinsky, R.Y. Tsien, M.H. Ellisman, Multicolor and electron microscopic imaging of connexin trafficking, Science, 296 (2002) 503507.

13. S.R. Adams, R.Y. Tsien, Preparation of the membrane-permeant biarsenicals FlAsH-EDT2 and ReAsH-EDT2 for fluorescent labeling of tetracysteine-tagged proteins, Nat Protoc, 3 (2008) 1527-1534.

14. S.R. Adams, R.E. Campbell, L.A. Gross, B.R. Martin, G.K. Walkup, Y. Yao, J. Llopis, R.Y. Tsien, New biarsenical ligands and tetracysteine motifs for protein labeling in vitro and in vivo: synthesis and biological applications, J Am Chem Soc, 124 (2002) 60636076.

15. R.W. Schwenk, E. Dirkx, W.A. Coumans, A. Bonen, A. Klip, J.F. Glatz, J.J. Luiken, Requirement for distinct vesicle-associated membrane proteins in insulin- and AMP-activated protein kinase (AMPK)induced translocation of GLUT4 and CD36 in cultured cardiomyocytes, Diabetologia, 53 (2010) 2209-2219.

16. M.M. van Oort, J.M. van Doorn, A. Bonen, J.F. Glatz, D.J. van der Horst, K.W. Rodenburg, J.J. Luiken, Insulin-induced translocation of CD36 to the plasma membrane is reversible and shows similarity to that of GLUT4, Biochim Biophys Acta, 1781 (2008) 61-71.

17. Y. Oligschlaeger, M. Miglianico, D. Chanda, R. Scholz, R.F. Thali, R. Tuerk, D.I. Stapleton, P.R. Gooley, D. Neumann, The recruitment of AMP-activated protein kinase to glycogen is regulated by autophosphorylation, J Biol Chem, 290 (2015) 11715-11728.

18. C. Hoffmann, G. Gaietta, A. Zurn, S.R. Adams, S. Terrillon, M.H. Ellisman, R.Y. Tsien, M.J. Lohse, Fluorescent labeling of tetracysteine-tagged proteins in intact cells, Nat Protoc, 5 (2010) 1666-1677.

19. C. Szent-Gyorgyi, B.F. Schmidt, Y. Creeger, G.W. Fisher, K.L. Zakel, S. Adler, J.A. Fitzpatrick, C.A. Woolford, Q. Yan, K.V. Vasilev, P.B. Berget, M.P. Bruchez, J.W. Jarvik, A. Waggoner, Fluorogen-activating single-chain antibodies for imaging cell surface proteins, Nat Biotechnol, 26 (2008) 235-240.

20. J. Holleran, D. Brown, M.H. Fuhrman, S.A. Adler, G.W. Fisher, J.W. Jarvik, Fluorogen-activating proteins as biosensors of cell-surface proteins in living cells, Cytometry A, 77 (2010) 776-782.

21. P.M. Kane, Targeting reversible disassembly as a mechanism of controlling V-ATPase activity, Curr Protein Pept Sci, 13 (2012) 117-123.

22. P.M. Kane, Disassembly and reassembly of the yeast vacuolar $\mathrm{H}(+)$-ATPase in vivo, $\mathrm{J}$ Biol Chem, 270 (1995) 17025-17032. 



\title{
Chapter 5
}

\section{Central role of dysassembly of vacuolar-type $\mathrm{H}^{+}$-ATPase in lipid-induced cardiomyopathy}

\author{
Yilin Liu, Amber Geomini, \\ Jan F.C. Glatz, Dietbert Neumann, \\ Joost J.F.P. Luiken ${ }^{\#}$
} University, P.O. Box 616, 6200 MD Maastricht, the Netherlands 


\begin{abstract}
Cardiac lipid metabolism is mainly regulated by CD36, the predominant membrane fatty acid transporter, which translocates from intracellular storage compartments (endosomes) to the sacolemma upon hormonal/mechanical stimuli. Upon overexposure of the heart to lipids, CD36 permanently relocates to the sarcolemma, initiating a vicious cycle of lipid-induced insulin resistance and cardiac dysfunction. Vacuolar $\mathrm{H}^{+}$-ATPase (v-ATPase, also known as endosomal proton pump) plays a key role in lipid-induced subcellular CD36 relocation, whereby lipid inhibits v-ATPase activity, resulting in endosomal alkalinization and expulsion of CD36 from the endosomes to the sarcolemma. Studies with rat models in vivo and cardiomyocytes in vitro suggest that the underlying mechanism of lipid-induced v-ATPase inhibition is the disassembly of v-ATPase into $V_{0}$ and $\mathrm{V}_{1}$ sub-complex which inactivates its activity. In the present study we investigated the ability of glucose, which was reported to induce v-ATPase re-assembly in yeast, as well as that of compounds with known protective effects against lipid-induced insulin resistance, such as A-769662 (a potent AMP-activated protein kinase (AMPK) activator) and eicosapentaenoic acid (EPA, an omega-3 fatty acid), on v-ATPase dynamics and subcellular CD36 distribution in cardiomyocytes. Our results indicate that high glucose concentrations induce reassembly of v-ATPase in lipid-overloaded cardiomyocytes, and thereby preserve v-ATPase function and endosomal CD36 retention. In contrast, 2-deoxy-D-glucose (2-DG) did not exert these beneficial actions, suggesting that the underlying mechanism of glucose-induced v-ATPase reassembly requires glucose metabolism. A-769662 also prevented v-ATPase dysfunction and excess CD36 translocation to the sarcolemma, thereby possibly implicating a role for phosphorylation of a specific subunit in the regulation of v-ATPase assembly/disassembly. In contrast, EPA was ineffective, suggesting that EPA might exert its beneficial effects through mechanisms independent of v-ATPase activity. Taken together, these data provide evidence for glucose and AMPK-mediated phosphorylation playing significant roles in the regulation of $\mathrm{v}$-ATPase activity in cardiomyocytes.
\end{abstract}




\section{Introduction}

Elevated cardiac LCFA uptake and myocardial lipid accumulation as the result of long-chain fatty acid (LCFA) oversupply have been associated with insulin resistance and contractile dysfunction $[1,2]$. Cardiac LCFA uptake is mainly regulated by CD36 (the predominant fatty acid transporter in the heart), which translocates from endosomes to the sarcolemma upon hormonal or mechanical stimuli [3]. Upon overexposure of the heart to lipids, CD36 mainly is found at the sarcolemma, initiating a vicious cycle of increased lipid uptake and lipid-induced insulin resistance, leading to cardiac dysfunction [4]. Previous work has provided evidence that CD36, but not GLUT4, was expelled from the endosomes upon endosomal alkalinization, suggesting that proper function of the endosomal proton pump (v-ATPase) is related to endosomal CD36 retention [5]. Studies in high-fat diet fed rats have shown that impairment of v-ATPase might play a key role in lipid-induced localization of CD36 at the sarcolemma, and subsequent cardiac insulin resistance and contractile dysfunction (Chapter 3). Specifically, in these rats the onset of sarcolemmal CD36 abundance, as well as increased LCFA uptake and decreased insulin signaling (consistent with earlier work [6]) was strongly associated with endosomal alkalinization, providing a novel link between v-ATPase functioning and cardiac insulin resistance (Chapter 3). Further investigations uncovered the causal link between v-ATPase inhibition and increased CD36-mediated LCFA uptake by genetic silencing or pharmacological inhibition of v-ATPase, which could be rescued by CD36 silencing (Chapter 3). Taken together, v-ATPase dysfunction appears to underly CD36 relocation to the sarcolemma and subsequent development of lipid-induced cardiomyopathy.

As a proton pump occurring in acidic organelles, v-ATPase is responsible for endosomal acidification $[7,8]$. V-ATPase is structurally divided into a cytosolic $\mathrm{V}_{1}$ subcomplex and a transmembrane $\mathrm{V}_{0}$ sub-complex, encompassing the ATP catalyzing activity and proton channel, respectively [8]. Studies in yeast and mammalian kidney cells have revealed that v-ATPase activity is mainly regulated via assembly and disassembly of the two sub-complexes [9-11]. These assembly/disassembly cycles were found to be regulated by glucose availability, as glucose deprivation caused v-ATPase disassembly, whereas glucose-enriched conditions favored assembly and hence restoration of organellar acidification [9-11]. Upon disassociation of the two subcomplexes, $\mathrm{V}_{1}$ and $\mathrm{V}_{0}$, $\mathrm{v}$-ATPase activity declines [12]. In line with the findings from yeast and kidney cells, we observed nutritional regulation of v-ATPase activity. Specifically, we found that besides glucose (as mentioned above) also availability of lipids regulates of v-ATPase assembly status. In more detail, lipid overload causes migration of the $\mathrm{V}_{1}$ sub-complex into the cytoplasm, thereby providing a mechanistic explanation for lipid-induced v-ATPase inhibition. The $\mathrm{V}_{1}$ sub-complex migration was observed by using 
both immunoprecipitation and subcellular fractionation methods (Chapter 3), and further confirmed microscopically (Chapter 4). Hence, all these observations revealed disassembly of v-ATPase as a key step towards lipid-induced cardiac insulin resistance and contractile dysfunction. Based on the re-assembly of v-ATPase in yeast upon increased glucose availability [9-11], we speculate that also in the mammalian heart, increased glucose levels might trigger v-ATPase assembly.

As the main regulator of intracellular and whole-body energy metabolism, AMPactivated protein kinase (AMPK) is an attractive therapeutic target for the treatment of type 2 diabetes. Upon its activation AMPK stimulates the process to generate ATP through its downstream substrates so as to restore the normal energy level. In this way AMPK activation improves insulin sensitivity and glucose homeostasis. Metformin is the most widely prescribed drug for treatment of type 2 diabetes worldwide [13]. Metformin is assumed to exert its beneficial effects primarily in the liver through indirect activation of AMPK $[14,15]$, although it has also been proposed that metformin inhibits hepatic gluconeogenesis via decreasing hepatic energy state, independently of the AMPK pathway [16]. In the diabetic heart, AMPK activation has been shown to improve insulin resistance by blocking the negative feedback loop in the insulin signaling pathway [17]. Yet, we do not know whether the beneficial effects of AMPK activation also extend to the regulation of $\mathrm{v}$-ATPase assembly in endosomes.

Fish oils, enriched in omega-3 fatty acids (among which eicosapentaenoic acid, EPA) have been demonstrated to improve whole body insulin resistance in rodent models of obesity and diabetes $[18,19]$. Furthermore, EPA (C20:5n-3) has been shown to increase glucose and fatty acid uptake, prevent sarcolemmal abundance of CD36, insulin resistance and loss of contractile activity in primary cardiomyocytes cultured under insulin resistant conditions, suggesting that EPA is beneficial to preserve cardiac function [20]. Yet, the mechanism how EPA prevents CD36 relocation to the sarcolemma, and subsequent cardiac insulin resistance and contractile dysfunction is unknown. Thus, it would be interesting to examine whether EPA would be able to regulate v-ATPase assembly status.

To test whether increased glucose availability could induce v-ATPase reassembly in cardiomyocytes upon lipid oversupply, and thereby re-activate v-ATPase activity, we investigated the effect of high glucose concentrations on the v-ATPase assembly/ disassembly state in lipid-overloaded cardiomyocytes by using both immunoprecipitation and subcellular fractionation approaches. Furthermore, we studied chloroquine accumulation and CD36 surface staining to see whether endosomal function (i.e., acidification) and CD36 relocation to the sarcolemma could be restored after glucose addition. Especially, we investigated whether glucose would exert these effects by itself or 
by a metabolite obtained after glycolytic conversion, by replacing glucose with the nonmetabolizable glucose analog 2-deoxy-D-glucose (2-DG). Furthermore, we included long-term treatment with EPA and AMPK stimulator A-769662 to explore other possible mechanisms in regulating assembly/disassembly of v-ATPase. Finally, we evaluated insulin signaling upon the various treatments applied in order to determine whether the potential changes in endosomal function and surface CD36 presence corresponded to alterations in insulin signaling.

\section{Methods}

\section{Isolation and Culturing of Primary Rat Cardiomyocytes}

Male Lewis rats, 200-250 grams, were purchased from Charles River laboratories. Cardiomyocytes were isolated from those rats by a Langendorff perfusion system as previously described [21].

\section{Culturing of HL-1 cardiomyocytes}

HL-1 cells were cultured in control medium as previously described [22]. High palmitate media was prepared as described in Chapter 3.

\section{Immunoprecipitation}

Immunoprecipitation of HL-1 cells was performed as described in Chapter 3.

\section{Subcellular Fractionation}

Primary rat cardiomyocytes or HL-1 cardiomyocytes were washed with cold PBS and collected with ice cold SET buffer (10 mM Tris, $2 \mathrm{mM}$ EDTA and $250 \mathrm{mM}$ sucrose). The cell lysates were obtained by a dounce homogenizer, which was followed by three cycles of freeze-thawing with liquid nitrogen. Subsequently, cell lysates were spun down at 500 $\mathrm{g}$ for $1 \mathrm{~min}$ to discard cell debris, the supernatant was thereafter centrifuged for $60 \mathrm{~min}$ at 200,000 g (Beckman Coulter, optima ${ }^{\text {Tm }}$ MAX-XP, CA, USA). The pellet, containing subcellular membranes, was re-suspended in $200 \mu \mathrm{l}$ of SET buffer. Additionally, the supernatant, representing the cytoplasm, was collected in $100 \mu \mathrm{l}$. After protein determination, samples were prepared for western blotting.

\section{Cell Lysis and Western Blotting}

Cell lysis and Western blotting were performed as described earlier [23]. The following primary antibodies were used: v-ATPase a2 and v-ATPase B2 (1: 1000 in 5\% BSA in TBST; Abcam), GAPDH (1: 2000 in 5\% BSA in TBST; Cell Signaling Technology), and Caveolin-3 (1:6000 in 1\% non-fat dry milk in TBST; BD Transduction Laboratories). Primary antibodies were detected by either anti-rabbit secondary antibody (1:2000 in 5\% non-fat dry milk in TBST; Cell Signaling Technology) or anti-mouse secondary antibody 
(1:20000 in TBST; Dako).

\section{Measurement of Cellular CHLQ Accumulation in Cultured Cardiomyocytes}

Cellular $\left[{ }^{3} \mathrm{H}\right]$ chloroquine accumulation were performed as mentioned in Chapter 3.

\section{CD36 Cell Surface Staining}

Colorimetric detection of CD36 at the sarcolemma using a HRP-linked secondary antibody was carried out as previously described [24].

\section{Statistics}

All data are presented as means \pm SEM. Statistical analysis was performed by using two-sided Student's t-test. P-values of less than 0.05 were considered statistically significant.

\section{Results}

Our earlier study has identified that lipid overexposure inhibited v-ATPase via dissociation of the soluble $\mathrm{V}_{1}$ sub-complex from the membrane-bound $\mathrm{V}_{0}$ sub-complex, thereby providing a mechanistic explanation of lipid-induced endosomal alkalinization (Chapter 3). Since reversible disassembly of v-ATPase was regulated by glucose in yeast $[8,25]$, we investigated whether glucose would exert this same action in the mammalian heart by culturing primary and HL-1 cardiomyocytes in control, high-palmitate, and high-palmitate with $25 \mathrm{mM}$ high glucose media, and subsequently using these cells for subcellular fractionation. In both cardiac cell models, we observed that the a 2 subunit as part of the membrane-bound $V_{0}$ sub-complex was entirely localized to the membrane fractions in all the conditions, whereas the $\mathrm{B} 2$ subunit as indicator of the soluble $\mathrm{V}_{1}$ subcomplex, was shifted from the membrane to the cytoplasmic fraction upon highpalmitate treatment compared to the control condition (Figure 1), in agreement with our previous observations (Chapter 3). Upon short-term glucose treatment, membranous B2 content was redistributed back by $30-40 \%$ to the integral membrane sub-complex $\mathrm{V}_{0}$ (Figure 1), indicating that also in cardiomyocytes glucose promoted reassembly of v-ATPase upon lipid oversupply.

To further investigate whether glucose addition would restore endosomal acidity and CD36 retention, both primary and HL-1 cardiomyocytes were exposed to short-term and long-term glucose for chloroquine accumulation assay and CD36 surface staining. As shown in earlier experiments (Chapter 3), pharmacological inhibition of v-ATPase by bafilomycin-A (BafA) caused $>80 \%$ decrease of $\mathrm{v}$-ATPase function in both primary and HL-1 cardiomyocytes (Figure 2). Consistent with our previous findings (Chapter 3), v-ATPase function was decreased by $40-50 \%$ upon high-palmitate treatment in primary and HL-1 cardiomyocytes. Importantly, this was restored by $20-30 \%$ upon long-term 

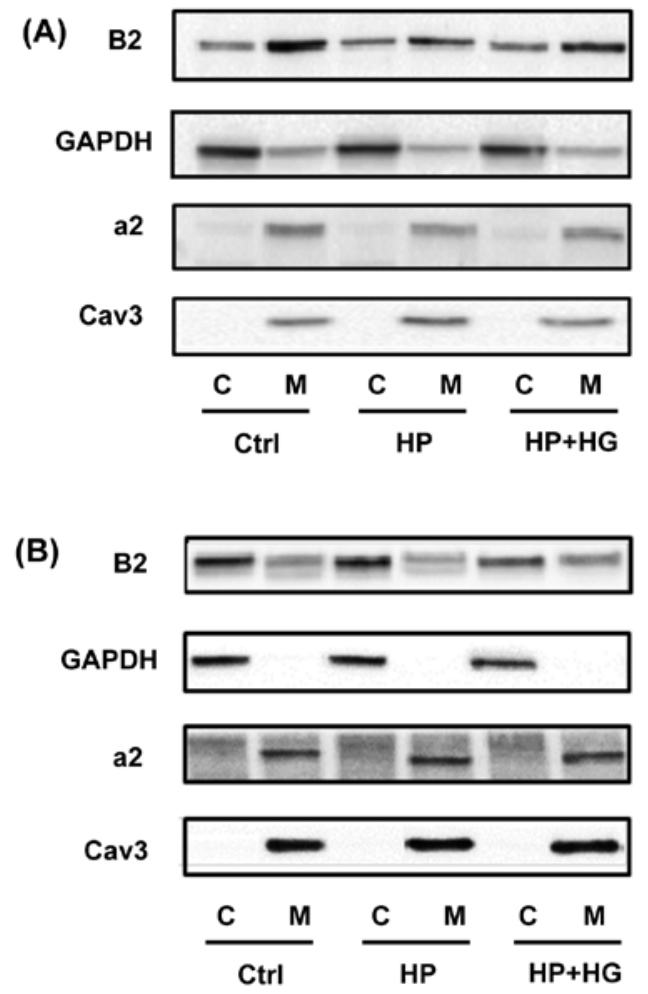

Figure 1. Assembly and disassembly of v-ATPase in cardiomyocytes as studied by subcellular fractionation. (A) HL-1 cardiomyocytes were treated either with serum-free depletion medium (ctrl) or depletion medium supplemented with $20 \mu \mathrm{M}$ palmitate and $50 \mathrm{nM}$ insulin (HP) for $16 \mathrm{~h}$. (B) Primary rat cardiomyocytes were treated either with control medium (ctrl; M199 supplemented with $5 \mathrm{mM}$ creatine monohydrate, $3.2 \mathrm{mM}$ carnitine hydrochloride, $3.1 \mathrm{mM}$ taurine, $20 \mu \mathrm{M}$ palmitate with palmitate:BSA=0.3:1) or HP medium (control medium with $200 \mu \mathrm{M}$ palmitate with palmitate:BSA=3:1) for $16 \mathrm{~h}$. The next day, one of the HP condition was treated with $25 \mathrm{mM}$ glucose (HP/HG) for $20 \mathrm{~min}$ in both HL-1 and primary cardiomyocytes, and subsequently used for subcellular fractionation. Contents of v-ATPase subunit a2 (a2) and v-ATPase subunit B2 (B2) were assessed by western blotting in both the cytoplasmic fraction (C) and the membrane fraction (M). Caveolin-3 and GAPDH were detected as the loading control of v-ATPase a2 and v-ATPase B2, respectively. Representative blots of 3 independent experiments are shown.

glucose incubation (Figure 2). In contrast, no alteration was seen upon short-term glucose incubation (20 min), nor upon short-term or long-term ( $\geq 16 \mathrm{~h}$ ) 2-DG incubation. As observed in primary cardiomyocytes, v-ATPase function was also decreased upon long-term insulin, EPA or insulin together with EPA incubations (Figure 2B), but not upon treatment with the AMPK activator, A-769662 [26]. Yet, A-769662 partially prevented the high-palmitate-induced impairment in v-ATPase function, whereas no effect was observed in control culturing conditions (Figure 2B). Collectively, glucose and A-769662 partially restored v-ATPase function upon lipid oversupply, indicating 
(A)
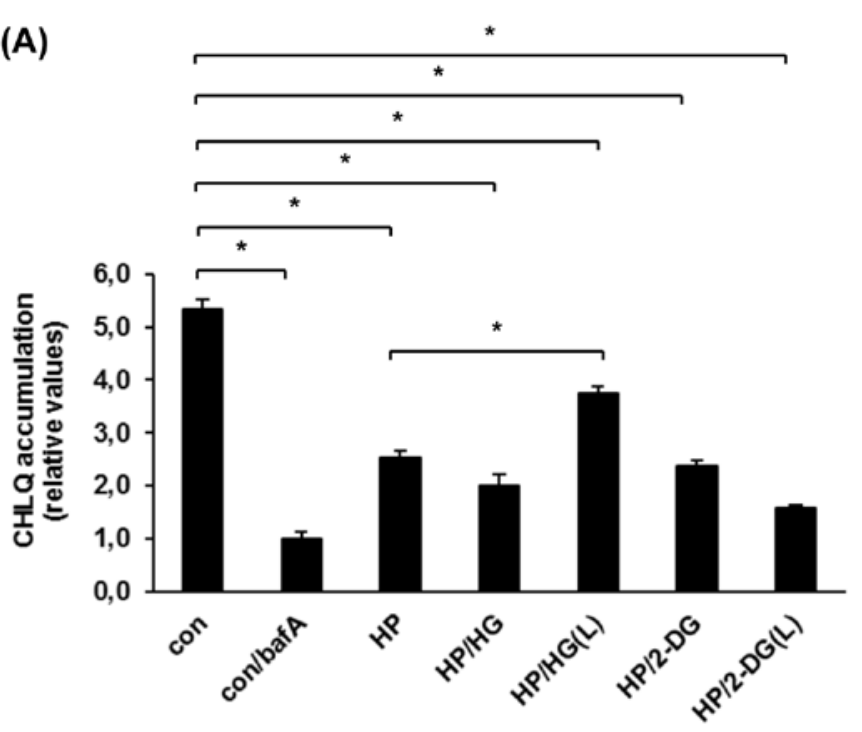

(B)
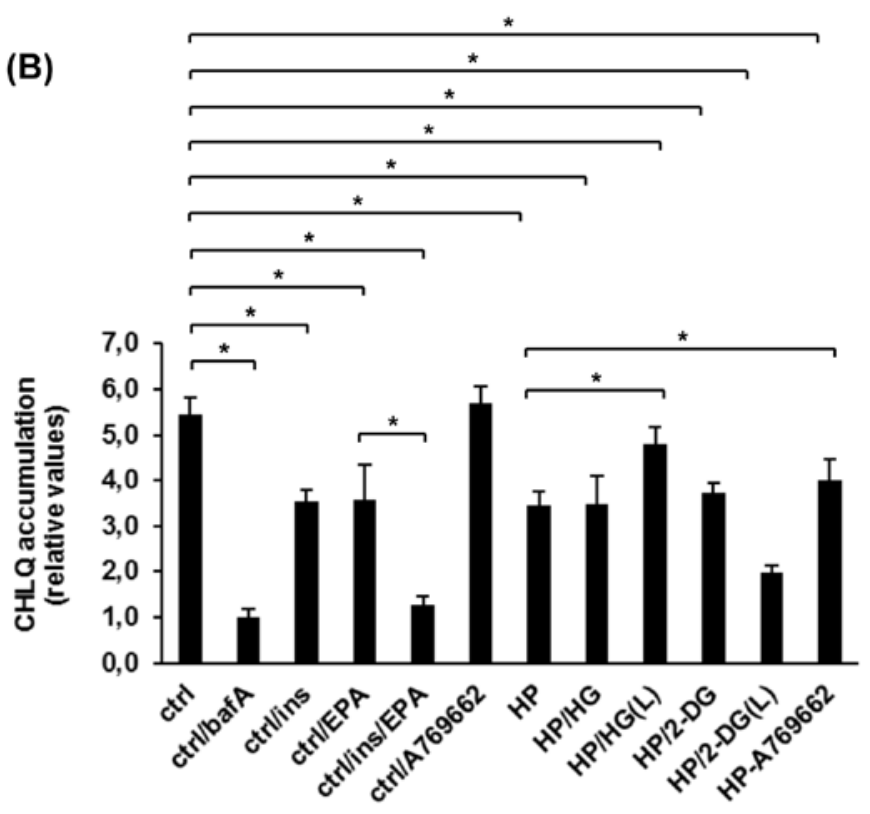

Figure 2. Chloroquine accumulation in primary and HL-1 cardiomyocytes upon different treatments. (A) HL-1 cardiomyocytes were treated with ctrl medium, HP medium, HP medium supplemented with $25 \mathrm{mM}$ glucose (HP/HG(L)) or with $25 \mathrm{mM}$ 2-deoxy-D-glucose (2-DG) (HP/2$\mathrm{DG}(\mathrm{L}))$ for $16 \mathrm{~h}$. (B) Primary rat cardiomyocytes were treated either with ctrl medium, ctrl medium supplemented with $50 \mathrm{nM}$ insulin (ctrl/ins), $200 \mu \mathrm{M}$ EPA (ctrl/EPA), insulin and EPA (ctrl/ins/EPA) or $100 \mu \mathrm{M}$ A769662 (ctrl/A769662), or treated with HP medium, HP/HG(L) medium, HP/2-DG(L) medium, or HP medium supplemented with $100 \mu \mathrm{M}$ A769662 (HP/A769662) for $24 \mathrm{~h}$. The next day, selected ctrl and HP conditions were treated with $100 \mathrm{nM}$ bafilomycin (ctrl/bafA), HG (HP/HG), or 2-DG (HP/2-DG) for $20 \mathrm{~min}$, and thereafter were ready for ${ }^{3}[\mathrm{H}]$ chloroquine accumulation assay in both primary and HL-1 cardiomyocytes . Values are displayed as mean \pm SEM $(n=3)$. ${ }^{\star} P<0.05$; N.S., not significant. 


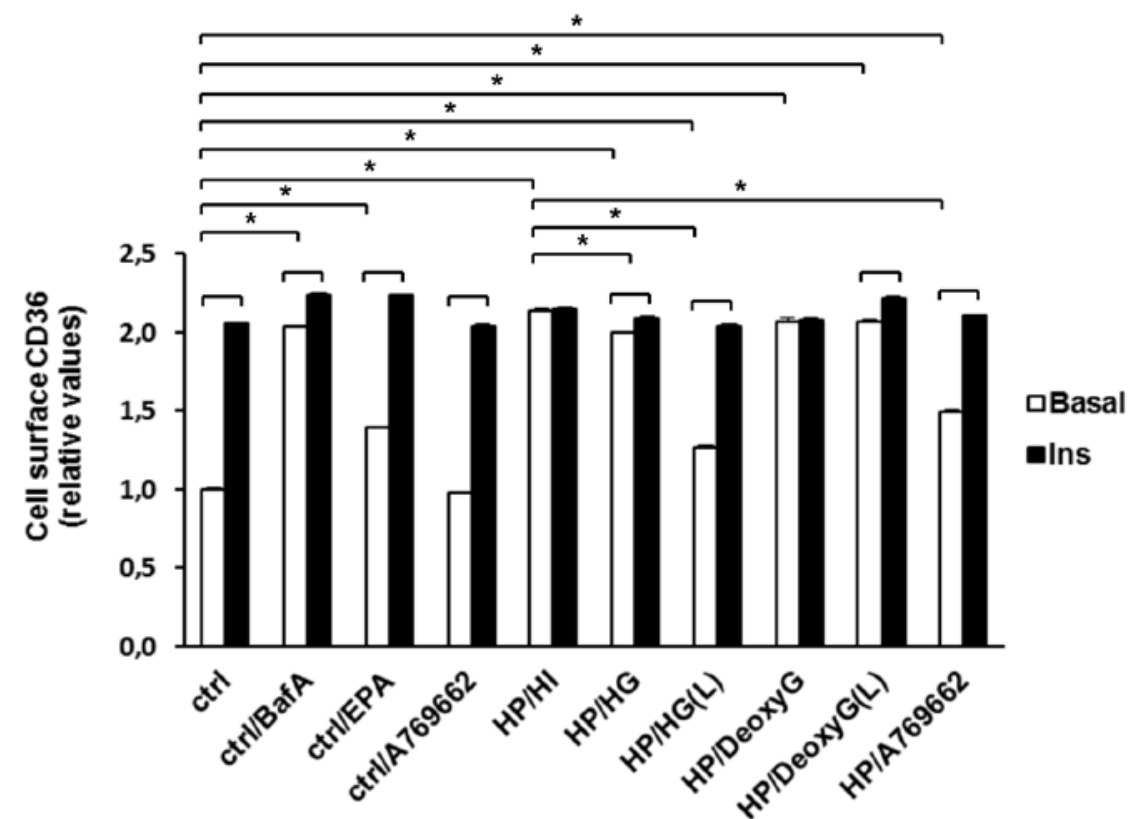

Figure 3. CD36 cell surface staining in HL-1 cardiomyocytes upon different treatments. Prior to CD36 cell surface staining, HL-1 cardiomyocytes were either treated with ctrl medium, ctrl/EPA medium, or ctrl/A769662, or treated with HP medium, HP/HG(L) medium, HP/2-DG(L) medium, or HP/A769662 medium for $16 \mathrm{~h}$. The next day, selected ctrl and HP conditions were treated with bafilomycin (ctrl/bafA), glucose (HP/HG) or 2-DG (HP/2-DG) for $20 \mathrm{~min}$, whereas the long-term treatments remained the same. Subsequently, cells were stimulated either with or without $200 \mathrm{nM}$ insulin for $30 \mathrm{~min}$ and immunochemically stained for sarcolemmal CD36 content. Values are displayed as mean \pm SEM. $(n=3){ }^{\star} P<0.05$; N.S., not significant.

beneficial roles for both compounds in mediating lipid-induced v-ATPase dysfunction.

Previously, we have shown that v-ATPase inhibition led to increased CD36 translocation from endosomes to the sarcolemma (Chapter 3). Here we assessed whether the effects of the various treatments on v-ATPase function in the absence and presence of high palmitate also extend to the regulation of surface CD36 content in HL-1 cells. High-palmitate culturing or long-term BafA treatment caused a 2-fold increase in sarcolemmal CD36 content at the cost of insulin-stimulated CD36 translocation. A 1.4fold increase in sarcolemmal CD36 content was also observed upon long-term treatment with EPA (Figure 3), yet insulin sensitivity of CD36 translocation was retained. In contrast, treatment of high palmitate-cultured cells with short-term glucose, short-term 2-DG, or long-term 2-DG, neither decreased the elevated sarcolemmal CD36 content, nor preserved insulin-stimulated CD36 translocation (Figure 3). Remarkably, long-term incubation with glucose or A-769662 partially prevented high-palmitate-induced CD36 translocation to the sarcolemma, whereas A-769662 did not show any effect in control culturing conditions (Figure 3). Taken together, these findings indicate that the beneficial 


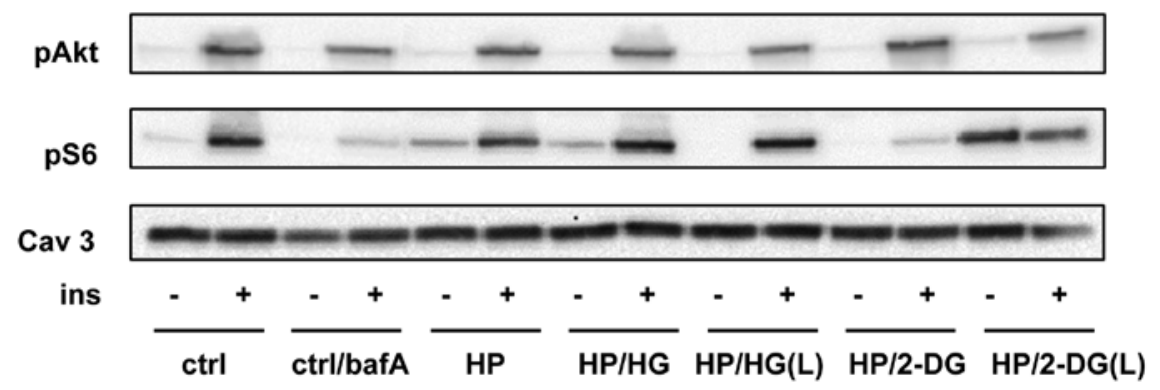

Figure 4. Effects on insulin signaling in HL-1 cardiomyocytes upon different treatments. HL-1 cardiomyocytes were treated with ctrl medium, $100 \mathrm{nM}$ bafilomycin (ctrl/bafA(L)), ctrl/EPA medium, HP medium, HP/HG(L) medium, or HP/2-DG(L) medium for $16 \mathrm{~h}$. The next day, selected ctrl and HP conditions were treated with glucose (HP/HG) or 2-DG (HP/2-DG) for 20 min, whereas the long-term treatments remained the same. Subsequently, cells were stimulated either with or without $200 \mathrm{nM}$ insulin for $30 \mathrm{~min}$ and harvested for western blotting analysis of phosphorylation of Akt (pAkt) and of ribosomal protein S6 (pS6). Caveolin 3 was detected as loading control. Representative blots of 3 independent experiments are shown.

effects of long-term glucose and A-769662 treatment on v-ATPase activity in lipidoverloaded cells each extend to the endosomal retention of CD36.

For evaluation of insulin signaling, phosphorylation of Akt (pAkt) and its substrate ribosomal protein S6 (pS6) were assessed. As positive control for induction of insulin resistance by v-ATPase inhibition, insulin-stimulated Akt and S6 phosphorylation were largely decreased upon bafA treatment (Figure 4). In line with previous observations (chapter 3), high-palmitate culturing induced a loss of insulin-stimulated phosphorylation of Akt and S6. 2-DG failed to preserve insulin signaling upon high-palmitate incubation (Figure 4). Interestingly, high glucose concentrations partially prevented this loss of insulin-stimulated S6 phosphorylation, but were not able to retain Akt phosphorylation during high-palmitate culturing (Figure 4). This partial restoration of insulin signaling by high glucose treatment may seem counter-intuitive given the maladaptively increased glucose levels during prolonged insulin resistance towards development of diabetes, but can be partially understood by the effects of high glucose at the level of v-ATPase function and associated subcellular CD36 distribution.

\section{Discussion}

The present study has revealed that lipid-induced disassembly of v-ATPase in endosomes of cardiomyocytes can be reversed by feeding glucose. Furthermore, our data indicate a possible role for AMPK in the reassembly of v-ATPase upon lipid oversupply. In addition, a link between v-ATPase function and endosomal CD36 retention in response to various biological conditions (high glucose concentrations, AMPK activation, EPA) was established. 


\section{$v$-ATPase function and high glucose concentrations}

Previously, we have identified disassembly of v-ATPase as a novel key event in the onset of insulin resistance upon lipid oversupply, suggesting v-ATPase as a putative lipid sensor in cardiomyocytes (Chapter 3). In yeast, the glucose-sensitive cycles of v-ATPase assembly/disassembly are rapid processes that occur within minutes, which excludes a required involvement of transcription factors and protein synthesis [27]. Here we investigated whether glucose regulation of assembly/disassembly is also occurring in the mammalian heart. Indeed, lipid-induced dissociation of v-ATPase was partially prevented by glucose treatment (Figure 1). Consistent with this, the presence of glucose also partially prevented the loss of v-ATPase function and endosomal CD36 retention (Figures 2 and 3), but the effect on preservation of insulin signaling is less clear (Figure 4) as will be further discussed below. The restoration of v-ATPase assembly and activity occurred only after long-term ( $\geq 16 \mathrm{~h}$ ) treatment of lipid-overloaded cardiomyocytes with glucose, whereas short-term (20 min) incubation, despite its rapid effects on v-ATPase reassembly, did not induce endosomal re-acidification or CD36 internalization. Perhaps, proton pumping rather than v-ATPase assembly presents the rate-limiting step in endosomal acidification. A resolution scale of hours between $20 \mathrm{~min}$ and $24 \mathrm{~h}$ of glucose addition is needed for further investigation so as to delineate the exact time of restoration of these two processes.

As for insulin signaling, we did not observe any effects of glucose on insulinstimulated Akt phosphorylation. Based on the ability of glucose to induce CD36 retention, we might expect a positive effect of glucose on the preservation of Akt phosphorylation, because CD36 retention would diminish myocellular diacylglycerol and ceramide levels, thereby relieving the lipid-induced brake on insulin signaling. Perhaps a high glucose availability may induce independent maladaptive (signaling) actions that would override the beneficial effects on CD36 retention. In a broader perspective, persistent high glucose levels could lead to a condition known as glucotoxicity, characterized by increased oxidative stress. Taking this a step further, increased concentrations of reactive oxygen species (ROS) might impair mitochondrial $\beta$-oxidation, so that lipids would be directed to storage pathways. Then, concomitant increases in diacylglycerols and ceramides would counterbalance the v-ATPase re-assembly-induced decreases in formation of these metabolites. A further paradoxical finding is the preservation of S6 phosphorylation. The dissociation between Akt phosphorylation and S6 phosphorylation is unexpected, but not impossible given the complex relationship between Akt activation and subsequent activation of mammalian target of rapamycin (mTOR), which then directly phosphorylates p70 S6 kinase and subsequently S6. mTOR is activated by multiple signaling routes, besides Akt, and possibly high glucose 
concentrations upregulate one of these routes. In conclusion, high glucose appears to restore several detrimental aspects of lipid-induced insulin resistance. Perhaps, insulinstimulated protein synthesis downstream of S6 phosphorylation is preserved, in contrast to insulin-stimulated glucose uptake downstream of the Akt-AS160 axis.

2-DG is known to be a stable glucose analogue that cannot be fully metabolized. 2-DG is transported into the cells by the hexose transporters and then phosphorylated into 2-DG-6-phosphate, which cannot be further metabolized by the glycolytic machinery. An earlier study in yeast has demonstrated that accumulation of glucose 6-phosphate was insufficient to induce reassembly of the v-ATPase, suggesting that glucose metabolism beyond formation of glucose 6-phosphate was required to maintain the intracellular pool of assembled v-ATPase [28]. In line with the results from yeast, 2-DG was unable to preserve $v$-ATPase function in cardiomyocytes, suggesting a lack of an effect of this compound on v-ATPase re-assembly also in mammalian cells. Consequently, 2-DG did not preserve endosomal CD36 retention in lipid-overexposed cardiomyocytes. Further studies in yeast reported that a physical interaction between $\mathrm{v}$-ATPase and the glycolytic enzyme aldolase regulated assembly/disassembly of v-ATPase [11, 29, 30]. Disrupting the interaction of aldolase with v-ATPase without affecting its glycolytic activity resulted in v-ATPase disassembly, whereas overexpressing of aldolase blocked v-ATPase disassembly upon glucose removal [11]. Taken together, these studies indicate that glucose metabolism plays a vital role in regulating assembly/ disassembly of v-ATPase in yeast. It would be interesting to explore the role of aldolase in glucose-mediated v-ATPase reassembly in lipid-overloaded cardiomyocytes.

\section{v-ATPase function and AMPK activations}

In this study, we also observed that the potent AMPK activator, A-769662 partially preserved the loss of v-ATPase function upon cardiac lipid oversupply (Figure 2), as well as the decrease of endosomal CD36 retention (Figure 3), indicating the beneficial role of AMPK in lipid-induced v-ATPase dysfunction, and subsequent cardiac insulin resistance and contractile dysfunction. Nevertheless, we did not observe the effect of A-769662 on v-ATPase function or CD36 translocation under control condition (Figures 2 and 3). Perhaps, v-ATPase is already maximally assembled during basal culturing, so that assembly-directed treatment is futile in this situation. A-769662 is a thienopyridone family of AMPK activators that directly activates AMPK in a similar manner to AMP and inhibits dephosphorylation of Thr172 [26, 31]. A-769662 improved glucose homeostasis in $o b / o b$ mice and whole-body fatty acid oxidation in primary rat hepatocytes [26]. Possibly, A-769662-induced AMPK activation results in phosphorylation of one of the v-ATPase subunits at a specific site, triggering the respective v-ATPase sub-complex to a conformational change that favors re-assembly with the other sub-complex. Whether 
v-ATPase assembly/disassembly is regulated at the level of v-ATPase phosphorylation needs further investigation.

\section{$v$-ATPase function and EPA}

The preventive effect of EPA on the development of insulin resistance and contractile dysfunction in the heart has been reported in numerous studies. Human studies also confirm the beneficial effects of EPA and other omega-3 fatty acids by improving glucose homeostasis and restoring defects in insulin signaling [32, 33]. However, EPA was not effective in preserving v-ATPase function in lipid-overloaded cardiomyocytes. Hence, the protective effects of EPA against lipid-induced loss of insulin sensitivity might be confined to upregulation of adipose triglyceride lipase (ATGL), as established earlier [19], which would then favor lipid disposal and subsequent de-inhibition of insulin signaling. In contrast, EPA decreased v-ATPase function and endosomal CD36 retention under the control condition. Interestingly, these results are in line with previous work, which have revealed a stimulatory effect of EPA on myocellular LCFA uptake. Based on the present results, EPA-stimulated LCFA uptake may occur via v-ATPase inhibition and increased CD36 translocation [20].

\section{Concluding remarks}

Taken together, v-ATPase is not only a lipid sensor, but also acts as a glucose sensor. Hence, whereas v-ATPase to our knowledge has not been linked before to the regulation of cardiac metabolism, these novel nutrient-sensing properties place v-ATPase at the forefront of determining cardiac substrate preference. Specifically, lipids cause v-ATPase disassembly, increased CD36 translocation, feeding forward to a vicious cycle of increased fatty acid uptake. Conversely, glucose induces v-ATPase assembly and CD36 internalization, which might further decrease fatty acid uptake and deposition of diacylglycerols and ceramides, thereby de-inhibiting GLUT4 translocation and glucose uptake. In conclusion, v-ATPase assembly/disassembly may act as a switch between myocellular LCFA and glucose uptake.

High glucose concentrations were chosen to provide proof-of-principle for the establishment of v-ATPase as a glucose sensor. As already argued, high glucose treatment would be a rather unfavorable strategy to combat lipid-induced insulin resistance. Therefore, other strategies to selectively upregulate glucose uptake would be more suitable in this respect. First of all, overexpression of GLUT1 and PKD1 could provide further proof-of-principle for the glucose-sensing properties of v-ATPase. Overexpression of GLUT1 will lead to increased basal glucose uptake [34]. PKD1 overexpression has been proven to induce GLUT4 translocation and glucose uptake without altering CD36 translocation and fatty acid uptake [35]. As a future step in therapy against lipid-induced 
insulin resistance, specific PKD1 activators could be employed to force myocytes to specifically take up glucose for subsequent v-ATPase reassembly. Unfortunately, because a number of compounds, such as alpha-agonists, known to induce PKD1 activation [36], also induce a variety of other unrelated pathways, specific PKD1 activators have not been identified yet.

Finally, it would be worth to investigate whether other AMPK activators besides A-769662 induce v-ATPase reassembly in lipid-overloaded myocytes. If so, it should be established whether this effect is indeed AMPK-dependent, for instance by studying AMPKa1a 2 double knockout models. As further evidence for a central role of v-ATPase in the insulin sensitizing action of AMPK activators, the beneficial effect of AMPK activators on the preservation of insulin signaling and insulin-stimulated glucose uptake should be re-assessed in cultured cells exposed to bafA so that v-ATPase is blocked downstream of disassembly. These cells are already insulin resistant upon culturing in media with low palmitate concentrations (chapter 3), and a putative lack of an effect of AMPK activators on the restoration of insulin sensitivity in this model would make $\mathrm{v}$-ATPase a key site in the insulin sensitizing action of AMPK activators. 


\section{References}

1. W.C. Stanley, G.D. Lopaschuk, J.G. McCormack, Regulation of energy substrate metabolism in the diabetic heart, Cardiovasc Res, 34 (1997) 2533 .

2. L.S. Szczepaniak, R.G. Victor, L. Orci, R.H. Unger, Forgotten but not gone: the rediscovery of fatty heart, the most common unrecognized disease in America, Circ Res, 101 (2007) 759-767.

3. J.F. Glatz, J.J. Luiken, A. Bonen, Membrane fatty acid transporters as regulators of lipid metabolism: implications for metabolic disease, Physiol Rev, 90 (2010) 367-417.

4. Y. Angin, L.K. Steinbusch, P.J. Simons, S. Greulich, N.T. Hoebers, K. Douma, M.A. van Zandvoort, W.A. Coumans, W. Wijnen, M. Diamant, D.M. Ouwens, J.F. Glatz, J.J. Luiken, CD36 inhibition prevents lipid accumulation and contractile dysfunction in rat cardiomyocytes, Biochem J, 448 (2012) 43-53.

5. L.K. Steinbusch, W. Wijnen, R.W. Schwenk, W.A. Coumans, N.T. Hoebers, D.M. Ouwens, W.A. Coumans, N.T. Hoebers, M. Diamant, A. Bonen, J.F. Glatz, J.J. Luiken, Differential regulation of cardiac glucose and fatty acid uptake by endosomal pH and actin filaments, Am J Physiol Cell Physiol, 298 (2010) C1549-1559.

6. D.M. Ouwens, M. Diamant, M. Fodor, D.D. Habets, M.M. Pelsers, M. El Hasnaoui, Z.C. Dang, C.E. van den Brom, R. Vlasblom, A. Rietdijk, C. Boer, S.L. Coort, J.F. Glatz, J.J. Luiken, Cardiac contractile dysfunction in insulin-resistant rats fed a high-fat diet is associated with elevated CD36-mediated fatty acid uptake and esterification, Diabetologia, 50 (2007) 1938-1948.

7. B. Ma, Y. Xiang, L. An, Structural bases of physiological functions and roles of the vacuolar H(+)-ATPase, Cell Signal, 23 (2011) 1244-1256.

8. P.M. Kane, Targeting reversible disassembly as a mechanism of controlling V-ATPase activity, Curr Protein Pept Sci, 13 (2012) 117-123.

9. P.M. Kane, The where, when, and how of organelle acidification by the yeast vacuolar $\mathrm{H}+-$ ATPase, Microbiol Mol Biol Rev, 70 (2006) 177191.

10. Y.Y. Sautin, M. Lu, A. Gaugler, L. Zhang, S.L. Gluck, Phosphatidylinositol 3-kinase-mediated effects of glucose on vacuolar H+-ATPase assembly, translocation, and acidification of intracellular compartments in renal epithelial cells, Mol Cell Biol, 25 (2005) 575-589.

11. M. Lu, D. Ammar, H. Ives, F. Albrecht, S.L. Gluck, Physical interaction between aldolase and vacuolar $\mathrm{H}+$-ATPase is essential for the assembly and activity of the proton pump, J Biol Chem, 282 (2007) 24495-24503.

12. P.M. Kane, C.T. Yamashiro, T.H. Stevens, Biochemical characterization of the yeast vacuolar H(+)-ATPase, J Biol Chem, 264 (1989) 19236-19244.

13. M.O. Goodarzi, M. Bryer-Ash, Metformin revisited: re-evaluation of its properties and role in the pharmacopoeia of modern antidiabetic agents, Diabetes Obes Metab, 7 (2005) 654-665.

14. R.J. Shaw, K.A. Lamia, D. Vasquez, S.H. Koo, N. Bardeesy, R.A. Depinho, M. Montminy, L.C. Cantley, The kinase LKB1 mediates glucose homeostasis in liver and therapeutic effects of metformin, Science, 310 (2005) 1642-1646.

15. S.H. Koo, L. Flechner, L. Qi, X. Zhang, R.A. Screaton, S. Jeffries, S. Hedrick, W. Xu, F. Boussouar, P. Brindle, H. Takemori, M. Montminy, The CREB coactivator TORC2 is a key regulator of fasting glucose metabolism, Nature, 437 (2005) 1109-1111.

16. M. Foretz, S. Hebrard, J. Leclerc, E. Zarrinpashneh, M. Soty, G. Mithieux, K. Sakamoto, F. Andreelli, B. Viollet, Metformin inhibits hepatic gluconeogenesis in mice independently of the LKB1/AMPK pathway via a decrease in hepatic energy state, J Clin Invest, 120 (2010) 2355-2369.

17. A. Ginion, J. Auquier, C.R. Benton, C. Mouton, J.L. Vanoverschelde, L. Hue, S. Horman, C. Beauloye, L. Bertrand, Inhibition of the mTOR/ p70S6K pathway is not involved in the insulinsensitizing effect of AMPK on cardiac glucose uptake, Am J Physiol Heart Circ Physiol, 301 (2011) H469-477.

18. L.H. Storlien, E.W. Kraegen, D.J. Chisholm, G.L. Ford, D.G. Bruce, W.S. Pascoe, Fish oil prevents insulin resistance induced by high-fat feeding in rats, Science, 237 (1987) 885-888.

19. P. Flachs, V. Mohamed-Ali, O. Horakova, M. Rossmeisl, M.J. Hosseinzadeh-Attar, M. Hensler, J. Ruzickova, J. Kopecky, Polyunsaturated fatty acids of marine origin induce adiponectin in mice fed a high-fat diet, Diabetologia, 49 (2006) 394-397.

20. V. Franekova, Y. Angin, N.T. Hoebers, W.A. Coumans, P.J. Simons, J.F. Glatz, J.J. Luiken, T.S. Larsen, Marine omega-3 fatty acids prevent myocardial insulin resistance and metabolic remodeling as induced experimentally by high insulin exposure, Am J Physiol Cell Physiol, 308 (2015) C297-307.

21. J.J. Luiken, F.A. van Nieuwenhoven, G. America, G.J. van der Vusse, J.F. Glatz, Uptake and 
metabolism of palmitate by isolated cardiac myocytes from adult rats: involvement of sarcolemmal proteins, J Lipid Res, 38 (1997) 745758.

22. R.W. Schwenk, E. Dirkx, W.A. Coumans, A. Bonen, A. Klip, J.F. Glatz, J.J. Luiken, Requirement for distinct vesicle-associated membrane proteins in insulin- and AMPactivated protein kinase (AMPK)-induced translocation of GLUT4 and CD36 in cultured cardiomyocytes, Diabetologia, 53 (2010) 22092219.

23. J.J. Luiken, D.P. Koonen, J. Willems, A. Zorzano, C. Becker, Y. Fischer, N.N. Tandon, G.J. Van Der Vusse, A. Bonen, J.F. Glatz, Insulin stimulates long-chain fatty acid utilization by rat cardiac myocytes through cellular redistribution of FAT/ CD36, Diabetes, 51 (2002) 3113-3119.

24. R.W. Schwenk, Y. Angin, L.K. Steinbusch, E. Dirkx, N. Hoebers, W.A. Coumans, A. Bonen, J.L. Broers, G.J. van Eys, J.F. Glatz, J.J. Luiken, Overexpression of vesicle-associated membrane protein (VAMP) 3, but not VAMP2, protects glucose transporter (GLUT) 4 protein translocation in an in vitro model of cardiac insulin resistance, J Biol Chem, 287 (2012) 37530-37539.

25. S. Bond, M. Forgac, The Ras/cAMP/protein kinase A pathway regulates glucose-dependent assembly of the vacuolar (H+)-ATPase in yeast, $J$ Biol Chem, 283 (2008) 36513-36521.

26. B. Cool, B. Zinker, W. Chiou, L. Kifle, N. Cao, M. Perham, R. Dickinson, A. Adler, G. Gagne, R. Iyengar, G. Zhao, K. Marsh, P. Kym, P. Jung, H.S. Camp, E. Frevert, Identification and characterization of a small molecule AMPK activator that treats key components of type 2 diabetes and the metabolic syndrome, Cell Metab, 3 (2006) 403-416.

27. P.M. Kane, Disassembly and reassembly of the yeast vacuolar $\mathrm{H}(+)$-ATPase in vivo, $\mathrm{J}$ Biol Chem, 270 (1995) 17025-17032.

28. K.J. Parra, P.M. Kane, Reversible association between the V1 and V0 domains of yeast vacuolar $\mathrm{H}+$-ATPase is an unconventional glucose-induced effect, Mol Cell Biol, 18 (1998) 7064-7074.

29. M. Lu, L.S. Holliday, L. Zhang, W.A. Dunn, Jr., S.L. Gluck, Interaction between aldolase and vacuolar H+-ATPase: evidence for direct coupling of glycolysis to the ATP-hydrolyzing proton pump, J Biol Chem, 276 (2001) 3040730413.

30. M. Lu, Y.Y. Sautin, L.S. Holliday, S.L. Gluck, The glycolytic enzyme aldolase mediates assembly, expression, and activity of vacuolar H+-ATPase, J Biol Chem, 279 (2004) 8732-8739.
31. M.J. Sanders, Z.S. Ali, B.D. Hegarty, R. Heath, M.A. Snowden, D. Carling, Defining the mechanism of activation of AMP-activated protein kinase by the small molecule A-769662, a member of the thienopyridone family, $\mathrm{J} \mathrm{Biol}$ Chem, 282 (2007) 32539-32548.

32. J. Chen, G.C. Shearer, Q. Chen, C.L. Healy, A.J. Beyer, V.B. Nareddy, A.M. Gerdes, W.S. Harris, T.D. O'Connell, D. Wang, Omega-3 fatty acids prevent pressure overload-induced cardiac fibrosis through activation of cyclic GMP/ protein kinase $\mathrm{G}$ signaling in cardiac fibroblasts, Circulation, 123 (2011) 584-593.

33. D. Fedor, D.S. Kelley, Prevention of insulin resistance by n-3 polyunsaturated fatty acids, Curr Opin Clin Nutr Metab Care, 12 (2009) 138146.

34. R. Liao, M. Jain, L. Cui, J. D’Agostino, F. Aiello, I. Luptak, S. Ngoy, R.M. Mortensen, R. Tian, Cardiac-specific overexpression of GLUT1 prevents the development of heart failure attributable to pressure overload in mice, Circulation, 106 (2002) 2125-2131.

35. E. Dirkx, G.J. van Eys, R.W. Schwenk, L.K. Steinbusch, N. Hoebers, W.A. Coumans, T. Peters, B.J. Janssen, B. Brans, A.T. Vogg, D. Neumann, J.F. Glatz, J.J. Luiken, Protein kinase-D1 overexpression prevents lipidinduced cardiac insulin resistance, $\mathrm{J} \mathrm{Mol} \mathrm{Cell}$ Cardiol, 76 (2014) 208-217.

36. E. Rozengurt, Protein kinase D signaling: multiple biological functions in health and disease, Physiology (Bethesda), 26 (2011) 23-33. 




\section{Chapter 6}

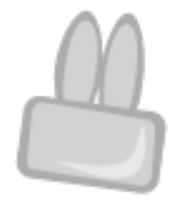

General discussion 


\section{General discussion}

\section{Introduction}

Diabetic cardiomyopathy is defined as diabetes-associated changes in the structure andfunctionofthemyocardium, suchasincreasedleftventricular(LV)mass, fibrosis, adilation of the ventricles, and decreased diastolic function in the presence of decreased or preserved systolic dysfunction [1-3], independent of hypertension or coronary artery disease [4, 5]. Myocardial lipotoxicity is a major contributor to the development of diabetic cardiomyopathy, as has been found in many rodent models and type 2 diabetic patients, and was linked to an enhanced long-chain fatty acid (LCFA) uptake and oxidation in the heart [6-10]. Cardiac lipid metabolism is largely regulated by CD36, which is the predominant fatty acid transporter in the heart and translocates from intracellular storage compartments to the sarcolemma upon hormonal and/or mechanical stimuli [11]. Upon overexposure of the heart to lipids, CD36 preferentially relocates to the sarcolemma, initiating a vicious cycle of increased fatty acid uptake, lipid-induced insulin resistance and cardiac dysfunction $[12,13]$. Nevertheless, how lipid oversupply could lead to the permanent relocation of CD36 from endosomes to the sarcolemma was unknown.

\section{Main findings in this thesis}

In this thesis, we investigated the putative role of vacuolar type $\mathrm{H}^{+}$-ATPase (v-ATPase) in lipid-induced subcellular CD36 relocation, and also examined the underlying mechanism. The main findings of this thesis are:

1. Cardiac lipid overload decreases v-ATPase function, thereby redistributing CD36 to the sarcolemma and increasing LCFA uptake that then precipitates as impaired insulin sensitivity and contractile dysfunction (Chapter 3 ).

2. Lipid oversupply of the heart causes disassembly of the v-ATPase molecule into its two sub-complexes with complex $\mathrm{V}_{0}$ remaining integral to the endosomal membrane and complex $\mathrm{V}_{1}$ shifting away into the cytoplasm, together resulting in inhibition of v-ATPase activity (Chapter 3).

3. Fluorescent labeling of fatty acid transporter CD36 by biarsenical dyes is a novel approach to visualize subcellular CD36 dynamics (Chapter 4).

4. Under basal conditions CD36 co-localizes with the integral membrane sub-complex $\mathrm{V}_{0}$ and the membrane associated sub-complex $\mathrm{V}_{1}$ of $\mathrm{v}$-ATPase, whereas in high-palmitate containing media the $\mathrm{V}_{1}$ sub-complex dissociates from CD36-containing vesicles, further supporting the concept that lipid-induced disassembly of the two sub-complexes of v-ATPase underlyes lipid-oversupply induced diabetic cardiomyopathy (Chapter 4). 
5. A high extracellular glucose concentration restores v-ATPase activity, endosomal CD36 localization, and insulin sensitivity in lipid-overloaded cardiomyocytes (Chapter 5).

In summary, lipid-induced v-ATPase inhibition in the heart has been disclosed as the culprit of the permanent CD36 relocation to the sarcolemma, and a potential strategy has been proposed to restore cardiac v-ATPase function in order to decrease myocardial lipid accumulation. To our knowledge v-ATPase has not yet been linked to cardiac metabolism. Below, these novel findings are reasoned from a broader point of view, especially in the context of cardiac energy substrate metabolism. Furthermore, possible mechanisms how lipids cause disassembly of v-ATPase are discussed. In addition, it is argued whether $\mathrm{v}$-ATPase itself or its function to maintain acidity in cellular environment is causal for CD36 relocation to the sarcolemma. Finally, based on our results, future perspectives are given as to how the presented work could be followed up.

\section{Cardiac substrate shift}

As described in Chapter 1, glucose and LCFA are the main cardiac energy substrates. In the healthy heart, there is a finely tuned distinctive balance between the utilization of both substrates with approximately $60 \%$ of the energy coming from LCFA and the remainder from glucose (and a minor contribution from lactate, not considered further here). The aberration of substrate utilization that is closely connected to cardiac disease has been described as substrate shift (Figure 1) [Glatz et al., Cardiovasc Drugs Ther 20 92006) 471-476]. A shift towards increased glucose utilization generally results in cardiac hypertrophy and heart failure $[14,15]$. In contrast, a shift to almost complete reliance on LCFA utilization is found to be associated with the development of diabetic cardiomyopathy $[9,16]$. The molecular mechanisms underlying these shifts in cardiac substrate utilization have been intensively investigated, but have remained far from being disclosed.

It is still widely accepted that the cardiac substrate balance is governed by the principles of the Randle cycle, a >50 year-old theory attributing a central role to acetyl-CoA, which intermediate is produced from both glucose and LCFA breakdown, resulting in inhibition of glucose oxidation by excess LCFA, and conversely, inhibition of LCFA oxidation by excess glucose $[17,18]$. The theory has been updated upon disclosing of the role of malonyl-CoA in the inhibition of LCFA oxidation [19]. With the discovery that myocellular glucose uptake, regulated by GLUT4 translocation from endosomal stores to the sarcolemma, provides the rate-limiting step in glucose utilization, the Randle cycle theory received a significant blow [20]. Subsequently, it was proposed that LCFA inhibit glucose utilization via 'toxic' lipid-intermediates, such as ceramides, which directly impair insulin signaling and GLUT4 translocation [20]. Also this theory contains significant flaws in that it does not take into account that subcellular CD36 translocation provides the main rate- 


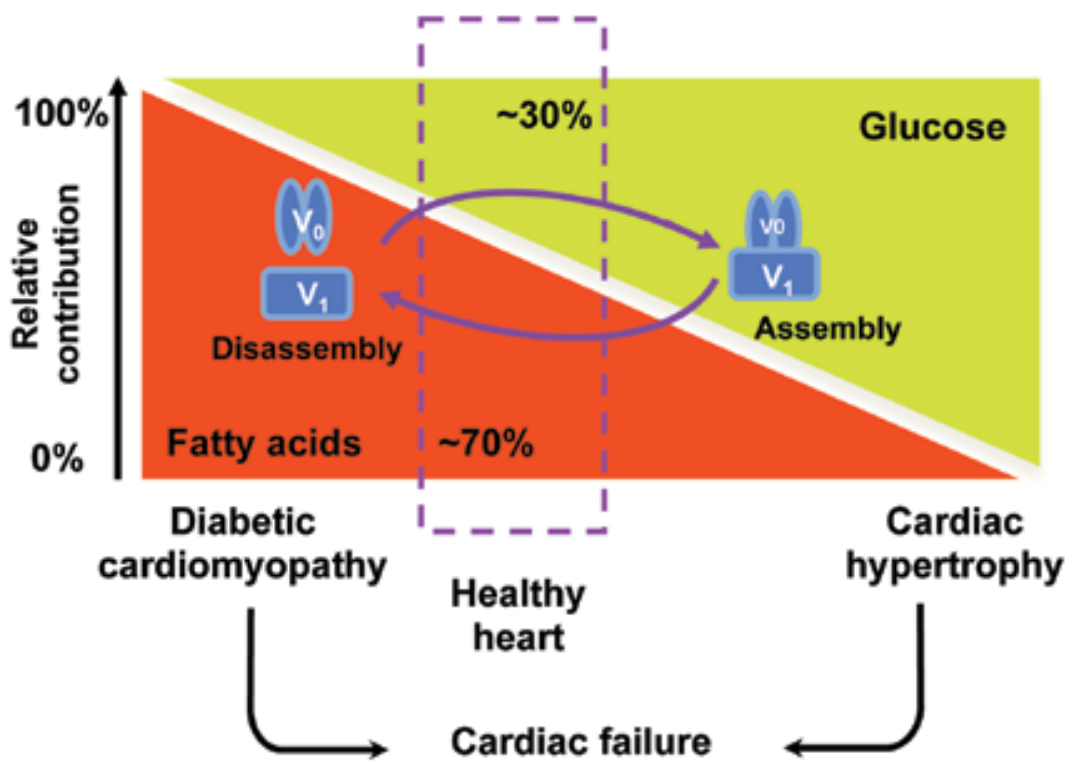

Figure 1. Substrate preference in heaIthy and failing heart and putative correlation with v-ATPase assembly status. Schematic presentation of substrate utilization in the heart, illustrating normal cardiac function is based on a balance between glucose and LCFA utilization as energy source. The preference of either glucose or LCFA utilization is a hallmark of cardiac hypertrophy and diabetic cardiomyopathy, respectively, ultimately leading to heart failure. Cardiac substrate balance might correspond to the status of assembly/disassembly of v-ATPase. Namely, increased LCFA utilization induces v-ATPase disassembly (Chapter 3), leading to sarcolemmal CD36 abundance and lipid accumulation in the heart. Vice versa, fully assembled v-ATPase would be expected to inhibit CD36 translocation and LFCA uptake therefore favoring glucose utilization. Both extremes are linked to disease. Based on our studies (Chapter 5), the glucose-induced (partial) assembly of v-ATPase prevents lipid-induced insulin resistance. Hence, we would predict a certain balance between assembled and disassembled v-ATPase in the healthy heart.

Source: Glatz et al., Cardiovasc Drugs Ther 20 92006:471-476

limiting step in LCFA uptake, and thereby confines the amount of 'toxic' lipid-intermediates [11]. Hence, the regulation of cardiac substrate preference currently lacks an integrative model that is in accordance with experimental observations. Based on the findings of this thesis, it can be proposed that v-ATPase regulates cardiac substrate preference via cycles of assembly/disassembly, that, on their turn, regulate CD36 translocation and thus the formation of 'toxic' lipid-intermediates that impair insulin signaling and GLUT4 translocation.

In more detail, our study has demonstrated that $\mathrm{v}$-ATPase disassembles into $\mathrm{V}_{0}$ and $\mathrm{V}_{1}$ sub-complexes upon lipid oversupply. Hence, v-ATPase may be seen as a lipid sensor in cardiomyocytes. V-ATPase inhibition by LCFA then causes further increased sarcolemmal CD36 abundance, in the long-term leading to lipid accumulation, decreased insulin signaling, and cardiac contractile dysfunction (Chapter 3). Hence, chronic v-ATPase $\mathrm{V}_{0} /$ 
$\mathrm{V}_{1}$ disassembly sets the heart on the road to diabetic cardiomyopathy.

Interestingly, previously it has been reported that in yeast such disassembly of v-ATPase induced by lipids can be reversed after glucose re-addition [21]. Likewise, in cardiomyocytes we observed that a high extracellular glucose availability restores v-ATPase function and decreases CD36 localization at the sarcolemma (Chapter 5). In line with our findings, it has been reported that overexpression of GLUT4 in diabetic $d b / d b$ mice not only restored glucose utilization, but significantly reduced the LCFA dependency of the heart and protected it from the development of contractile dysfunction [22]. These data indicate that in diabetic animal models increased glucose utilization effectively rebalances the cardiac metabolism (away from the exclusive use of LCFA), thereby preventing the development of cardiomyopathy. Hence, existing evidence suggests regulatory features of glucose, presumably by favoring $\mathrm{V}_{0} / \mathrm{V}_{1}$ assembly and thus maintenance of $\mathrm{v}$-ATPase function. As a result, v-ATPase may also act as a glucose sensor in cardiomyocytes. The corollary is that the cardiac glucose-fatty acid substrate balance then may be determined by a balance between assembly and disassembly of v-ATPase, with an optimal cardiac function being associated with a certain set point of distribution of assembly/disassembly of v-ATPase (Figure 1). Vice versa, a disruption of the balance between assembly and disassembly of $\mathrm{v}$-ATPase might be tightly linked to changes in cardiac substrate metabolism, leading to the development of cardiac diseases.

Further to this hypothetical mechanism outlined above, v-ATPase might be a promising therapeutic target to rectify a disruption of the cardiac substrate balance. Our current study has identified v-ATPase disassembly-induced alterations in cardiac LCFA metabolism to play a causal role in the development of diabetic cardiomyopathy (Chapter 3). In a therapeutic perspective and in accordance to our data (chapter 5), a 'forced' myocellular glucose uptake could operate the switch to lead from an excess LCFA utilization towards the glucose-fatty acid balance point thereby counteracting the known consequences of lipid oversupply in the heart. Such 'forced' glucose uptake, without changing LCFA uptake, seems feasible. For instance, it has been shown that protein kinase-D1 (PKD1) is selectively involved in contraction-activated GLUT4 but not CD36 translocation [23]. Additionally, in our in vitro model of cardiac insulin resistance overexpression of vesicle-associated membrane protein 3 (VAMP3), acting downstream of PKD, has been shown not only to improve inhibition of insulin-stimulated GLUT4 translocation, but also normalize CD36 distribution [24]. Thus, pharmaceutical upregulation of the PKD-VAMP3 axis could be an attractive approach to increase specifically glucose uptake, and subsequently restore cardiac substrate preference and contractile function in the lipid-overloaded heart. 


\section{Possible mechanisms regulating v-ATPase assembly and disassembly}

As described in Chapters 3 to 5, our studies with cardiomyocyte models show that high palmitate containing media inhibit v-ATPase function via its disassembly into the $\mathrm{V}_{0}$ and $V_{1}$ sub-complexes. High glucose counteracts palmitate by effectuating v-ATPase assembly. The question arises how palmitate or glucose is signaling to v-ATPase to induce assembly/disassembly. Regarding to the characteristics of assembly/disassembly, such mechanism would need to occur rapidly (minutes), most likely be reversible. Protein palmitoylation would be an attractive potential regulatory process as explained below.

In general, protein palmitoylation is the covalent attachment of palmitate molecules to proteins, which is widely recognized as a form of post-translational modification of proteins. In contrast to the closely related myristoylation (covalent attachment of a $\mathrm{C}_{14}$ fatty acid group), which is considered to be an irreversible protein modification, palmitoylation is readily reversible, and therefore compatible with short-term regulation. The reversible covalent bond occurs between palmitate and cysteine residues of proteins via a thioester linkage in most cases, and less frequently to serine and threonine residues of proteins $[25,26]$. These cysteines are usually part of a consensus motif recognized by specific enzymes, named palmitoyl-transferases (PATs). In more detail, dynamic palmitoylation is an enzymatic reaction, which requires, besides the (PATs), also palmitoylprotein thioesterases (PPTs) for palmitoylation and depalmitoylation. Moreover, it has been shown that palmitoylation regulates the subcellular localization $[27,28]$, stability [29], membrane interactions [28], as well as subcellular trafficking [25, 30] of proteins, and thereby modifies their functions. For many proteins, cycles of palmitoylation and depalmitoylation occur throughout their lifetime.

With respect to v-ATPase and palmitoylation, virtually no information is available. Literature search yielded only one conference abstract describing palmitoylation of $\mathrm{v}$-ATPase. Specifically, palmitoylation of the al subunit of the v-ATPase $\mathrm{V}_{0}$-subcomplex at Cys-25 was described to regulate interaction of $v$-ATPase with clathrin assembly complexes [31]. This interaction was previously identified as being essential for v-ATPase trafficking from endoplasmic reticulum (where it is synthesized) to the lysosomal membrane to allow assembly and activation $[32,33]$. In neuronal cells from mice with a deficiency in the PPT isoform-1, depalmitoylation is suppressed, which causes a mistargeting of the al subunit to the plasma membrane, thereby suppressing v-ATPase assembly and acidification of endosomes/lysosomes [31]. Overall, this study shows that PPT deficiency-induced palmitoylation of v-ATPase leads to its disassembly and loss of endosomal acidification.

The implication of this report is that it supports our hypothesis that v-ATPase palmitoylation is a feasible mechanism for v-ATPase disassembly, loss of endosomal acidification and increased CD36 translocation to the cell surface. Perhaps, increased 
palmitate supply will provide more substrate for the PATs, which then drives increased palmitoylation of all myocellular proteins with a palmitoylation consensus motif. Among these is the v-ATPase subunit a1, which upon increased palmitoylation at Cys-25, leaves the v-ATPase complex, thereby inducing $\mathrm{V}_{0} / \mathrm{V}_{1}$ disassembly. To investigate this possible mechanism, the Cys-25 residue could be subjected to site-directed mutagenesis, which subsequently might prevent $\mathrm{v}$-ATPase depalmitoylation and thus its disassembly, and render the lipid overloaded cardiomyocytes resistant to lipid-induced insulin resistance.

The mechanism by which glucose regulates $\mathrm{v}$-ATPase reassembly currently is unknown. From studies in yeast, a direct physical coupling of glycolysis to v-ATPase has been deduced as the mechanism by which glucose may regulate v-ATPase activity [34, 35 ]. In line with these results, we observed that glucose, but not 2-deoxy-D-glucose (2DG), induces the assembly of v-ATPase (Chapter 5). Therefore, glucose metabolism is required to stimulate reassembly of v-ATPase, and further restore v-ATPase function and endosomal CD36 retention (Chapter 5). Given the homology of v-ATPase in mammals and yeast [21], future studies should investigate the possible role of glycolysis in mediating v-ATPase assembly and activity.

\section{Mechanism of CD36 translocation: Involvement of endosomal alkalinization}

In Chapter 3, we have identified impairment of v-ATPase activity as the cause of lipidinduced CD36 relocation to the sarcolemma, and subsequent cardiac insulin resistance and contractile dysfunction. V-ATPase impairment was achieved by either long-term bafilomycin-A treatment or by knockdown of the B2 subunit of v-ATPase. Both these pharmacological and genetic strategies induce the loss of endosomal acidification. Hence, it might be concluded that v-ATPase inhibition induces the translocation of CD36 to the sarcolemma via endosomal alkalinization. On the other hand, the experiments in this thesis do not exclude the possibility that endosomal alkalinization is a collateral event and that $\mathrm{pH}$ changes are not required for $\mathrm{CD} 36$ translocation. In this respect, v-ATPase has been shown to serve as a docking site for both AMPK and mammalian target of rapamycin (mTOR), and thereby coordinate both anabolic and catabolic processes [36]. This latter functioning of v-ATPase could be influenced by palmitate and glucose signaling events, thereby affecting endosomal acidification. Conversely, the assembly status of v-ATPase that is linked to endosomal $\mathrm{pH}$ likely impacts on these signaling cascades.

Interestingly, earlier experiments of our research group have revealed that not only bafilomycin-A led to CD36 translocation, but that also the proton ionophore monensin exerted this same action [37]. Monensin has been shown to cause endosomal alkalinization due to the increased permeability of membranes for protons $[38,39]$, and therefore is acting independent of $\mathrm{v}$-ATPase. These earlier experiments may indicate the crucial involvement of endosomal alkalinization in CD36 translocation. Inevitably such 
conclusion leads to the following question: How does endosomal alkalinization induce CD36 translocation? Perhaps, endosomal alkalinization will modify charges on endosomal membrane phospholipids, which may impact on phospholipid-protein interactions, and subsequently on formation of protein complexes within the endosomal membrane. Another possibility is that the alterations in phospholipid charges induce curvature of the endosomal membranes, which then sets the stage for budding of CD36-containing vesicles from the endosomes. Clearly, disclosing the role of endosomal alkalinization in lipidinduced increased CD36 translocation in cardiomyocytes warrants further studies.

\section{Future perspectives}

Given that v-ATPase inhibition plays a crucial role in lipid-induced insulin resistance and contractile dysfunction of the heart, the suggestion arises that compounds capable of re-activating v-ATPase may protect the heart during lipid overload. Once identified, such agents may form the basis of novel therapies to combat lipid-induced cardiomyopathy. Such drug discovery endeavor likely requires more detailed investigations on the structural organization and post-translational modification of v-ATPase. Currently, v-ATPase is a greatly underexplored protein. Most likely, its complexity (v-ATPase consists of 14 subunits in a complex molecular arrangement) contributes to the lack of structural insight on v-ATPase. Apart from the palmitoylation-driven disassembly, other putative mechanisms of v-ATPase regulation are worth being investigated. One such mechanism is phosphorylation, as v-ATPase has been shown to be phosphorylated by AMPK [40], which is furthermore known to interact directly with mTOR and v-ATPase at the surface of endosomes [36].

To facilitate such investigations v-ATPase knockout mice may be generated by deleting one of the subunits of v-ATPase. Such mouse model would be expected to display myocardial insulin resistance and contractile dysfunction already on a low fat diet. The currently only available model is the a3-deficient $o c / o c$ mouse, which displays osteopetrosis [41]. Unfortunately, the a3 subunit is not expressed in the heart, rendering this mouse model less suitable for studies on the role of v-ATPase in lipid-induced cardiomyopathy. Yet the heterogeneity of v-ATPase subunits between different tissues also opens the perspective of tissue-specific modulation of v-ATPase activity. Transgenic mouse models overexpressing v-ATPase also might be a suitable research model for future investigations. Namely, these mice should be suited to test whether v-ATPase upregulation would be protective against lipid-induced cardiomyopathy. However, an experimental limitation of such approach is the fact that the generation of $\mathrm{v}$-ATPase overexpressing mice is a challenge because it would require the overexpression of each of the 14 subunits. On the other hand, it may be speculated that only one of these subunits is the limiting factor for the total protein complex generation. Therefore, studies on the transcriptional regulation or the 
subunit assembly mechanism of v-ATPase will be of importance to provide further insight.

Finally, the involvement of v-ATPase in lipid-induced insulin resistance should also be investigated in skeletal muscle. Given that CD36 translocation is similarly regulated in heart and muscle, especially with respect to signaling processes involved [11], it is to be expected that v-ATPase plays a similar role in the regulation of lipid metabolism in skeletal muscle as in the heart. In view of the important (quantitative) role of skeletal muscle on whole body glucose disposal, v-ATPase re-activating strategies would not only be effective against lipid-induced cardiomyopathy, but potentially also suitable to cure muscular insulin resistance and thus alleviate symptoms of type 2 diabetes. 


\section{References}

1. G.C. Fonarow, P. Srikanthan, Diabetic cardiomyopathy, Endocrinol Metab Clin North Am, 35 (2006) 575-599, ix.

2. D.L. Severson, Diabetic cardiomyopathy: recent evidence from mouse models of type 1 and type 2 diabetes, Can J Physiol Pharmacol, 82 (2004) 813-823.

3. D.S. Bell, Diabetic cardiomyopathy, Diabetes Care, 26 (2003) 2949-2951.

4. H. Bugger, E.D. Abel, Rodent models of diabetic cardiomyopathy, Dis Model Mech, 2 (2009) 454466.

5. F.S. Fein, Diabetic cardiomyopathy, Diabetes Care, 13 (1990) 1169-1179.

6. Y.T. Zhou, P. Grayburn, A. Karim, M. Shimabukuro, M. Higa, D. Baetens, L. Orci, R.H. Unger, Lipotoxic heart disease in obese rats: implications for human obesity, Proc Natl Acad Sci U S A, 97 (2000) 1784-1789.

7. J.J. Wright, J. Kim, J. Buchanan, S. Boudina, S. Sena, K. Bakirtzi, O. Ilkun, H.A. Theobald, R.C. Cooksey, K.V. Kandror, E.D. Abel, Mechanisms for increased myocardial fatty acid utilization following short-term high-fat feeding, Cardiovasc Res, 82 (2009) 351-360.

8. D.M. Ouwens, C. Boer, M. Fodor, P. de Galan, R.J. Heine, J.A. Maassen, M. Diamant, Cardiac dysfunction induced by high-fat diet is associated with altered myocardial insulin signalling in rats, Diabetologia, 48 (2005) 1229-1237.

9. S. Sharma, J.V. Adrogue, L. Golfman, I. Uray, J. Lemm, K. Youker, G.P. Noon, O.H. Frazier, H. Taegtmeyer, Intramyocardial lipid accumulation in the failing human heart resembles the lipotoxic rat heart, FASEB J, 18 (2004) 16921700 .

10. A.R. Wende, E.D. Abel, Lipotoxicity in the heart, Biochim Biophys Acta, 1801 (2010) 311-319.

11. J.F. Glatz, J.J. Luiken, A. Bonen, Membrane fatty acid transporters as regulators of lipid metabolism: implications for metabolic disease, Physiol Rev, 90 (2010) 367-417.

12. S.L. Coort, D.M. Hasselbaink, D.P. Koonen, J. Willems, W.A. Coumans, A. Chabowski, G.J. van der Vusse, A. Bonen, J.F. Glatz, J.J. Luiken, Enhanced sarcolemmal FAT/CD36 content and triacylglycerol storage in cardiac myocytes from obese zucker rats, Diabetes, 53 (2004) 1655-1663.

13. [13] D.M. Ouwens, M. Diamant, M. Fodor, D.D. Habets, M.M. Pelsers, M. El Hasnaoui, Z.C. Dang, C.E. van den Brom, R. Vlasblom, A. Rietdijk, C. Boer, S.L. Coort, J.F. Glatz, J.J. Luiken, Cardiac contractile dysfunction in insulin-resistant rats fed a high-fat diet is associated with elevated CD36-mediated fatty acid uptake and esterification, Diabetologia, 50 (2007) 1938-1948.

14. L.C. Heather, N.J. Howell, Y. Emmanuel, M.A. Cole, M.P. Frenneaux, D. Pagano, K. Clarke, Changes in cardiac substrate transporters and metabolic proteins mirror the metabolic shift in patients with aortic stenosis, PLoS One, 6 (2011) e26326.

15. L.C. Heather, M.A. Cole, C.A. Lygate, R.D. Evans, D.J. Stuckey, A.J. Murray, S. Neubauer, K. Clarke, Fatty acid transporter levels and palmitate oxidation rate correlate with ejection fraction in the infarcted rat heart, Cardiovasc Res, 72 (2006) 430-437.

16. H. Bugger, E.D. Abel, Molecular mechanisms for myocardial mitochondrial dysfunction in the metabolic syndrome, Clin Sci (Lond), 114 (2008) 195-210.

17. J.R. Neely, H.E. Morgan, Relationship between carbohydrate and lipid metabolism and the energy balance of heart muscle, Annu Rev Physiol, 36 (1974) 413-459.

18. G.D. Lopaschuk, J.R. Ussher, C.D. Folmes, J.S Jaswal, W.C. Stanley, Myocardial fatty acid metabolism in health and disease, Physiol Rev, 90 (2010) 207-258.

19. L. Hue, H. Taegtmeyer, The Randle cycle revisited: a new head for an old hat, Am J Physiol Endocrinol Metab, 297 (2009) E578-591.

20. R.G. Shulman, F. Hyder, D.L. Rothman, Lactate efflux and the neuroenergetic basis of brain function, NMR Biomed, 14 (2001) 389-396.

21. P.M. Kane, Targeting reversible disassembly as a mechanism of controlling V-ATPase activity, Curr Protein Pept Sci, 13 (2012) 117-123.

22. D.D. Belke, T.S. Larsen, E.M. Gibbs, D.L. Severson, Altered metabolism causes cardiac dysfunction in perfused hearts from diabetic (db/db) mice, Am J Physiol Endocrinol Metab, 279 (2000) E1104-1113.

23. E. Dirkx, R.W. Schwenk, W.A. Coumans, N. Hoebers, Y. Angin, B. Viollet, A. Bonen, G.J. van Eys, J.F. Glatz, J.J. Luiken, Protein kinase D1 is essential for contraction-induced glucose uptake but is not involved in fatty acid uptake into cardiomyocytes, J Biol Chem, 287 (2012) 58715881.

24. R.W. Schwenk, Y. Angin, L.K. Steinbusch, E. Dirkx, N. Hoebers, W.A. Coumans, A. Bonen, J.L. Broers, G.J. van Eys, J.F. Glatz, J.J. Luiken, Overexpression of vesicle-associated membrane protein (VAMP) 3, but not VAMP2, protects glucose transporter (GLUT) 4 protein 
translocation in an in vitro model of cardiac insulin resistance, J Biol Chem, 287 (2012) 37530-37539.

25. C. Aicart-Ramos, R.A. Valero, I. RodriguezCrespo, Protein palmitoylation and subcellular trafficking, Biochim Biophys Acta, 1808 (2011) 2981-2994.

26. M.D. Resh, Covalent lipid modifications of proteins, Curr Biol, 23 (2013) R431-435.

27. A.I. Magee, L. Gutierrez, I.A. McKay, C.J. Marshall, A. Hall, Dynamic fatty acylation of p21N-ras, EMBO J, 6 (1987) 3353-3357.

28. C. Salaun, J. Greaves, L.H. Chamberlain, The intracellular dynamic of protein palmitoylation, J Cell Biol, 191 (2010) 1229-1238.

29. M.E. Linder, R.J. Deschenes, Palmitoylation: policing protein stability and traffic, Nat Rev Mol Cell Biol, 8 (2007) 74-84.

30. Y. Fukata, M. Fukata, Protein palmitoylation in neuronal development and synaptic plasticity, Nat Rev Neurosci, 11 (2010) 161-175.

31. M. Bagh, G. Chandra, S. Peng, Z. Zhang, A. Mukherjee, A lysosomal targeting defect of $\mathrm{VOa} 1$ suppresses V-ATPase activity elevating lysosomal $\mathrm{pH}$ in Ppt1-/- mice: amelioration by NtBuHA, FASEB J, 29 (2015) 1.

32. Q. Liu, Y. Feng, M. Forgac, Activity and in vitro reassembly of the coated vesicle $(\mathrm{H}+)$-ATPase requires the $50-\mathrm{kDa}$ subunit of the clathrin assembly complex AP-2, J Biol Chem, 269 (1994) 31592-31597.

33. M.T. Drake, Y. Zhu, S. Kornfeld, The assembly of AP-3 adaptor complex-containing clathrincoated vesicles on synthetic liposomes, Mol Biol Cell, 11 (2000) 3723-3736.

34. M. Lu, L.S. Holliday, L. Zhang, W.A. Dunn, Jr., S.L. Gluck, Interaction between aldolase and vacuolar H+-ATPase: evidence for direct coupling of glycolysis to the ATP-hydrolyzing proton pump, J Biol Chem, 276 (2001) 3040730413.

35. M. Lu, D. Ammar, H. Ives, F. Albrecht, S.L. Gluck, Physical interaction between aldolase and vacuolar $\mathrm{H}+$-ATPase is essential for the assembly and activity of the proton pump, J Biol Chem, 282 (2007) 24495-24503.
36. C.S. Zhang, B. Jiang, M. Li, M. Zhu, Y. Peng, Y.L. Zhang, Y.Q. Wu, T.Y. Li, Y. Liang, Z. Lu, G. Lian, Q. Liu, H. Guo, Z. Yin, Z. Ye, J. Han, J.W. Wu, H. Yin, S.Y. Lin, S.C. Lin, The lysosomal v-ATPaseRagulator complex is a common activator for AMPK and mTORC1, acting as a switch between catabolism and anabolism, Cell Metab, 20 (2014) 526-540.

37. L.K. Steinbusch, W. Wijnen, R.W. Schwenk, W.A. Coumans, N.T. Hoebers, D.M. Ouwens, W.A. Coumans, N.T. Hoebers, M. Diamant, A. Bonen, J.F. Glatz, J.J. Luiken, Differential regulation of cardiac glucose and fatty acid uptake by endosomal pH and actin filaments, Am J Physiol Cell Physiol, 298 (2010) C1549-1559.

38. W.F. Boss, D.J. Morre, H.H. Mollenhauer, Monensin-induced swelling of Golgi apparatus cisternae mediated by a proton gradient, Eur J Cell Biol, 34 (1984) 1-8.

39. T. Yoshimori, A. Yamamoto, Y. Moriyama, M. Futai, Y. Tashiro, Bafilomycin A1, a specific inhibitor of vacuolar-type $\mathrm{H}(+)$-ATPase, inhibits acidification and protein degradation in lysosomes of cultured cells, J Biol Chem, 266 (1991) 17707-17712.

40. R. Alzamora, M.M. Al-Bataineh, W. Liu, F. Gong, H. Li, R.F. Thali, Y. Joho-Auchli, R.A. Brunisholz, L.M. Satlin, D. Neumann, K.R. Hallows, N.M. Pastor-Soler, AMP-activated protein kinase regulates the vacuolar $\mathrm{H}+$-ATPase via direct phosphorylation of the A subunit (ATP6V1A) in the kidney, Am J Physiol Renal Physiol, 305 (2013) F943-956.

41. K. Henriksen, C. Flores, J.S. Thomsen, A.M. Bruel, C.S. Thudium, A.V. Neutzsky-Wulff, G.E. Langenbach, N. Sims, M. Askmyr, T.J. Martin, V. Everts, M.A. Karsdal, J. Richter, Dissociation of bone resorption and bone formation in adult mice with a non-functional V-ATPase in osteoclasts leads to increased bone strength, PLoS One, 6 (2011) e27482. 

Appendices
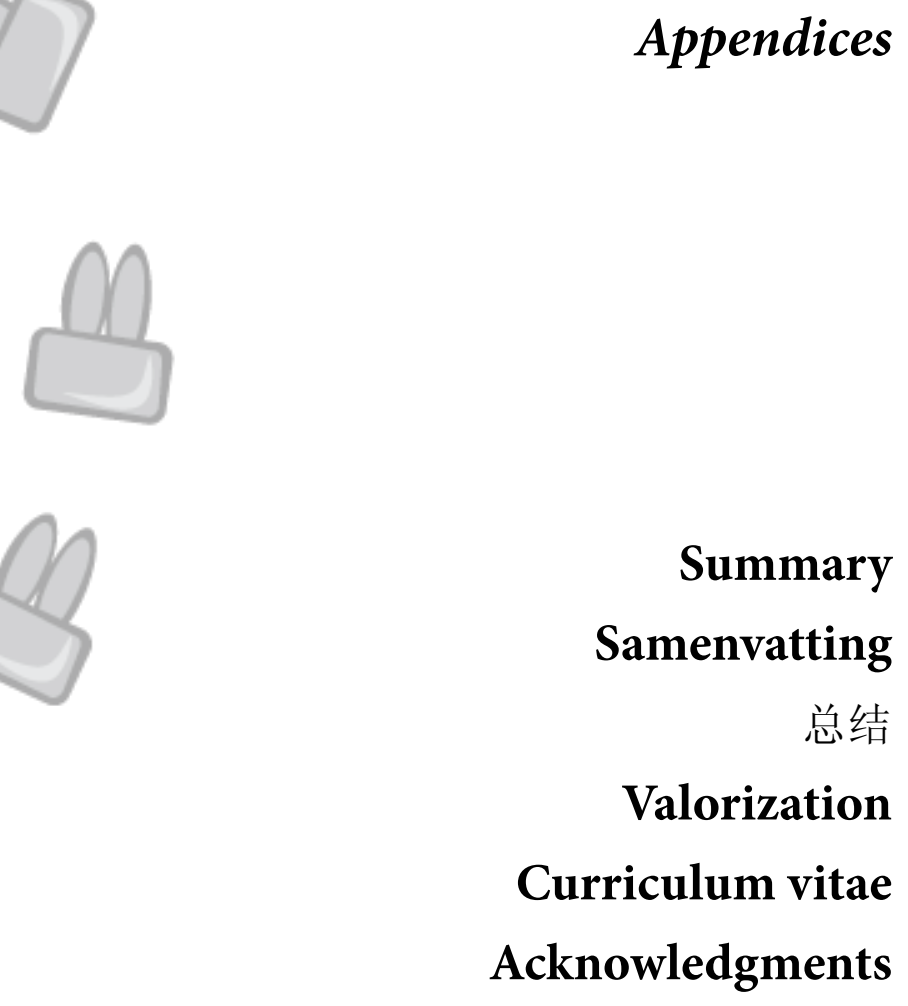


\section{Summary}

CD36 is the predominant membrane fatty acid transporter in the heart and a main contributor to the regulation of cardiac fatty acid uptake and metabolism. The basis of this regulation is that upon specific stimuli, such as increased contraction or hormones (e.g., insulin), CD36 translocates from intracellular storage compartments to the sarcolemma to increase fatty acid uptake. However, during overexposure of the heart to lipids (e.g., diet with a high fat content) CD36 preferentially locates to the sarcolemma, thereby stimulating myocardial fatty acid uptake and utilization, initiating a vicious cycle of excess intracellular lipid storage, lipid-induced insulin resistance and ultimately cardiac contractile dysfunction. As a result, CD36 is a key player in lipid-induced cardiac insulin resistance and contractile dysfunction, which subsequently leads to type 2 diabetes and diabetic cardiomyopathy.

It has been generally accepted that CD36-mediated fatty acid uptake is a primary contributor to myocellular lipid accumulation that causes insulin resistance, which in turn leads to contractile dysfunction. Although several key mediators, in particular lipid metabolites, have been proposed to contribute downstream of CD36 to the onset of insulin resistance which then results in contractile dysfunction, some observations have indicated that lipids themselves can directly induce contractile dysfunction (reviewed in Chapter 2). Several pieces of evidence have been reported to support this hypothesis, but the exact underlying molecular mechanism has not yet been unraveled. Therefore, the aim of this thesis was to investigate the upstream events leading CD36 to preferentially locate at the sarcolemma upon exposure to high fat, so as to further disclose the molecular mechanism of lipid-induced cardiac contractile dysfunction.

Focus was on the endosomes that constitute the major intracellular CD36 storage compartment, and in particular on vacuolar-type $\mathrm{H}^{+}$-ATPase (v-ATPase) that is responsible for endosomal acidification. Preliminary evidence had shown that v-ATPase is malfunctioning during lipid overload, leading to CD36 expulsion to the sarcolemma. Indeed, exposure of rat hearts in vivo to high lipids, or of either rodent or human cardiomyocyte cultures to high lipid media led to endosomal alkalinization, followed by insulin resistance, and subsequently decreased sarcomere shortening (Chapter 3). Genetic silencing or pharmacological inhibition of v-ATPase in cardiomyocytes was found to reduce insulin sensitivity, insulin-stimulated glucose uptake, and cardiac contractility, which were rescued by CD36 silencing. We further disclosed the potential underlying mechanism: this involves the disassembly of the two sub-complexes of v-ATPase, with one sub-complex $\left(\mathrm{V}_{0}\right)$ remaining at the endosomal membrane and the other sub-complex $\left(\mathrm{V}_{1}\right)$ dissociating into the cytoplasm. This disassembly caused loss of enzymatic activity (proton pumping) of $\mathrm{v}$-ATPase, thereby decreasing endosomal acidification. The resulting 
alkalinization of the endosomal lumen induced CD36 relocation from endosomes to the sarcolemma, subsequently leading to insulin resistance, and ultimately contractile dysfunction of the heart.

To further study the association between CD36 and v-ATPase, Chapter 4 establishes a novel approach to image CD36 fluorescently based on the introduction of a tetracysteine sequence in the extracellular loop and application of biarsenical dyes. Three CD36 mutants were constructed, each encoding the tetracysteine motif at a different site within the extracellular domain of CD36. One of these mutants showed normal biological function, and microscopic analysis after staining with a biarsenical dye was in agreement with the expected endosomal localization of CD36. The two sub-complexes of v-ATPase were stained via a classical immunofluorescent approach using distinct antibodies against specific subunits within each sub-complex. The $\mathrm{V}_{0}$ sub-complex co-localized with CD36 under both low-palmitate and high-palmitate culturing conditions, whereas colocalization of $\mathrm{V}_{1}$ sub-complex and $\mathrm{CD} 36$ was only observed under low-palmitate culturing condition. These data further confirmed disassembly of v-ATPase upon lipid oversupply. Taken together, this new approach provides an alternative methodology to study CD36 trafficking dynamics.

The reversible assembly/disassembly of the two sub-complexes appears as a main regulatory mechanism of v-ATPase activity in both yeast and kidney cells. In yeast, assembly/disassembly cycles have been reported to be regulated by glucose availability, with glucose-enriched conditions favoring assembly and hence increased endosomal acidification. Taking this a step further, we observed that high glucose concentrations may stimulate v-ATPase reassembly in lipid-overloaded cardiomyocytes (Chapter 5). Since 2-deoxy-D-glucose did not induce v-ATPase reassembly, glucose did not act directly but possibly via a glycolytic metabolite. In the meanwhile, A-769662, a potent AMP-activated protein kinase (AMPK) activator, also exerted beneficial effects on restoring the loss of lipid-induced v-ATPase dysfunction and preventing excess CD36 translocation to the sarcolemma, thereby suggesting the possible involvement of AMPK-mediated phosphorylation at a specific subunit in the regulation of v-ATPase assembly/disassembly.

Collectively, this thesis offers new insights on the molecular mechanisms by which excess lipids ultimately lead to cardiac contractile dysfunction and diabetic cardiomyopathy. Loss of v-ATPase activity appears to be a key player in the initiation of the sequence of events that lead to this pathological condition. As another result, strategies to re-activate v-ATPase might restore endosomal CD36 retention, cardiac insulin sensitivity and contractile function, which eventually would combat lipid-induced cardiomyopathy. 


\section{Samenvatting}

CD36 is het belangrijkste membraangebonden vetzuur-transporterende eiwit in het hart en het draagt in grote mate bij aan de regulatie van de vetzuuropname en het vetzuurmetabolisme. Deze regulatie vindt als volgt plaats: na blootstelling aan specifieke stimuli, zoals een toegenomen contractie van de hartspier of een verandering in de bloedspiegel van hormonen (bijv. insuline) migreert dit CD36 van intracellulaire opslagplaatsen (endosomen) naar het sarcolemma (plasmamembraan) alwaar de vetzuuropname snelheid kan worden vergroot. Wanneer het hart echter wordt geconfronteerd met een grote hoeveelheden vetten (lipiden) - bijvoorbeeld na een voeding met een hoog vetgehalte - blijkt het CD36 zich voornamelijk te bevinden op het sarcolemma, waardoor de vetzuuropname in rustcondities al is verhoogd is. Het gevolg hiervan is dat zich een overschot aan vetten in de cel opstapelt, waarna er op termijn insuline resistentie optreedt en tenslotte een afname van de hartfunctie. CD36 vervult dus een sleutelrol bij lipidegeïnduceerde insulineresistentie en de daarmee gepaard gaande afnemende contractie van het hart, wat vervolgens kan leiden tot zogenaamde diabete cardiomyopathie.

Alhoewel voor verschillende lipide-metabolieten is gesuggereerd dat zij de verbindende schakel zijn tussen de CD36-gemedieerde vetzuuropname en het ontstaan van insulineresistentie en afname van de contractiekracht van het hart, is het ook mogelijk dat deze lipide-metabolieten direct verantwoordelijk zijn voor de afname van de hartfunctie (zonder tussenkomst van insuline resistentie) (Hoofdstuk 2). Het preciese onderliggende moleculaire mechanisme is echter nog niet bekend. Het belangrijkste doel van dit proefschrift was dan ook om de gebeurtenissen voorafgaand aan de bovengenoemde CD36 re-lokalisatie naar het sarcolemma als gevolg van verhoogde blootstelling aan lipiden te onderzoeken, en daarmee de eerste stappen van het moleculaire mechanisme van lipide-geïnduceerd hartfalen verder te ontrafelen.

De focus van dit onderzoek lag op het endosomale compartiment dat de belangrijkste intracellulaire opslagplaats van CD36 vormt, en met name op het eiwit vacuole-specifiek $\mathrm{H}^{+}$-ATPase (v-ATPase) dat verantwoordelijk is voor verzuring van de endosomen. De eerste experimenten lieten zien dat gedurende blootstelling van hartspiercellen aan grote hoeveelheden lipiden v-ATPase niet goed functioneert (de endosomen worden minder zuur) en leidt tot verplaatsing van CD36 naar het sarcolemma. In vervolgexperimenten bleek dat na genetische manipulatie van hartspiercellen waarbij de hoeveelheid v-ATPase is gehalveerd, of bij farmacologische remming van het v-ATPase zowel de insuline gevoeligheid, de insuline-gestimuleerde glucose opname als de contractiekracht afnamen. Deze afname kon worden hersteld door met genetische technieken het CD36 gehalte te verlagen (Hoofdstuk 3). Hierna konden we het onderliggende mechanisme ontrafelen: de afname van v-ATPase activiteit bleek samen te gaan met het uiteenvallen van v-ATPase in 
twee sub-complexen waarbij het ene sub-complex $\left(\mathrm{V}_{0}\right)$ in het endosomale membraan blijft en het andere sub-complex $\left(\mathrm{V}_{1}\right)$ wegdrijft in het cytoplasma. Het wegvallen van de verbinding tussen deze twee sub-complexen veroorzaakt verlies van enzymatische activiteit (protonpomp functie) van v-ATPase, waardoor de endosomale zuurgraad afneemt. De resulterende alkalinizering van de endosomale binnenruimte induceert de verplaatsing van CD36 van endosomen naar het sarcolemma, hetgeen vervolgens leidt tot insuline resistentie en afname van contractiele functie van de hartspiercellen.

Om het verband tussen CD36 en v-ATPase verder te onderzoeken, is een nieuwe benadering opgezet om CD36 via fluorescentie in beeld te brengen. Deze benadering is gebaseerd op het inbrengen van een tetracysteine sequentie in het extracellulaire domein van CD36 en het labelen met bi-arsenicum kleurstoffen (Hoofdstuk 4). Drie CD36 mutanten werden geconstrueerd, waarbij het tetracysteine motief op verschillende plaatsen in het extracellulaire domein van CD36 werd ingebracht. Eén van deze mutanten toonde een normale biologische functie, en ook het microscopische kleuringspatroon na kleuring met het bi-arsenicum was in overeenstemming met de verwachte endosomale lokalisatie van CD36. De twee sub-complexen van v-ATPase werden gekleurd via een klassieke immunofluorescente benadering met behulp van verschillende antilichamen gericht tegen specifieke sub-units van elk sub-complex. De lokalisatie van het $\mathrm{V}_{0}$ subcomplex overlapte met die van CD36 onder zowel lage als hoge lipide-concentraties in het kweekmedium, terwijl co-lokalisatie tussen het $\mathrm{V}_{1}$ sub-complex en CD36 alleen optrad bij lage lipide-concentraties. Deze resultaten bevestigen het mechanisme van het uiteenvallen van v-ATPase tijdens een verhoogde blootstelling aan lipiden. Bovendien vormt deze nieuwe experimentele benadering een alternatieve methode om verandering in de cellulaire lokalisatie van CD36 te bestuderen.

Het omkeerbare proces van assemblage en uiteenvallen van de twee sub-complexen van v-ATPase is al een bekend regelmechanisme voor de activiteit van v-ATPase in gist. Cycli van assemblage en uiteenvallen van de sub-complexen worden in gist gereguleerd door de mate van beschikbaarheid van glucose, waarbij hoge glucose spiegels assemblage bevorderen alsook endosomale verzuring. Gebruikmakend van deze inzichten hebben wij ook waargenomen dat in hartspiercellen blootgesteld aan hoge lipide-spiegels de toevoeging van een hoge glucose concentratie de re-assemblage van v-ATPase weer bewerkstelligt (Hoofdstuk 5). Aangezien 2-deoxy-D-glucose deze re-assemblage van $\mathrm{v}$-ATPase niet bleek te herstellen, is het aannemelijk dat glucose dit effect teweegbrengt via een metaboliet uit de glycolyse. Ook vonden we dat A-769662, een sterke activator van AMP-activated protein kinase (AMPK) het lipide-geïnduceerde verlies aan v-ATPase functie en de daaraan gekoppelde excessieve verplaatsing van CD36 naar de sarcolemma eveneens herstelt. Dit laatste doet vermoeden dat v-ATPase assemblage wordt gereguleerd door AMPK-gemedieerde fosforylering van een specifieke sub-unit. 
Samengevat verschaft dit proefschrift een aantal nieuwe inzichten in de moleculaire mechanismen waarbij verhoogde lipide-spiegels uiteindelijk leiden tot verlies van de contractiekracht van het hart en het ontstaan van diabete cardiomyopathie. Een afgenomen v-ATPase activiteit blijkt een hoofdoorzaak te zijn bij de initiatie van een keten aan veranderingen die tesamen leiden tot dit ziektebeeld. Hiermee samenhangend kunnen strategiën die erop gericht zijn om de activiteit van v-ATPase te herstellen bijdragen aan de internalisatie van CD36 naar endosomale opslag, waarna de insuline gevoeligheid en de contractiele functie van het hart hersteld kunnen worden en in breder perspectief diabete cardiomyopathie bestreden kan worden. 


\section{总结}

CD36是存在于心脏的主要膜脂肪转运体, 通过调节心脏脂肪的吸收和代谢从而调节心肌细胞的脂 质水平。在增加收缩或激素（例如胰岛素）等特定条件的刺激下, CD36从细胞内储存室转运至肌膜以 增加脂肪酸的摄取。然而, 当心脏过度暴露于脂质的情况下 (例如, 摄入具有高脂肪含量的饮食) , CD36倾向于定位在肌膜, 从而刺激心肌脂肪酸的摄取和利用而引发细胞内多余脂质的存储, 脂质引 发的胰岛素抵抗并最终导致心肌收缩功能障碍的恶性循环。因此, CD36在脂质诱导的心肌胰岛素抵抗 和收缩功能障碍中扮演着关键的角色, 这一系列的病变随后导致 2 型糖尿病和糖尿病心肌疾病的发 生。

CD36介导的脂肪酸摄取被普遍认为是导致肌细胞脂质堆积的主要原因。大量的脂肪酸摄取导致胰 岛素抵抗, 并最终诱发心肌收缩功能障碍。虽然很多假设提出几个关键介质, 特别是脂质代谢物介导 了 CD36下游的信号通路从而诱发胰岛素抵抗, 并导致心肌收缩功能障碍, 然而也有另外一些观察表 明, 脂质本身可以直接诱导心肌收缩功能障碍（第2章中综述提及）。一些支持这些假说的证据已经 被发现, 但确切的分子机制依然没有被解开。因此, 本论文的目的是通过研究导致CD36倾向于定位 在肌膜的上游信号通路, 从而进一步揭开脂质引起的心肌收缩功能障碍的分子机制。

第三章的重点集中在主要构成细胞内CD36储藏室的内涵体, 尤其是负责内涵体酸化的液泡型氢离 子三磷酸腺苷酶 （v-ATPase）。初步证据表明, v-ATPase在脂质过载时出现功能性障碍, 导致CD36 定位在肌膜。事实上, 无论是体内实验使大鼠的心脏暴露于高脂质条件下, 或是体外实验将啮齿动物 或人的心肌细胞培养在高浓度脂质培养液中都能够发现高脂质能够导致内涵体碱化, 随之引发胰岛素 抵抗, 并且伴随肌节收缩的降低（第3章）。通过基因敲除或药理性地抑制心肌细胞的v-ATPase均使 胰岛素的敏感性、胰岛素刺激的葡萄糖摄取以及心肌的收缩力降低, 然而这些水平的降低可通过基因 敲除CD36而恢复正常水平。进一步的研究发现导致v-ATPase失去其正常功能的潜在机制涉及到 v-ATPase两个子复合体的分离。在高脂质的条件下, 其中一个子复合体 $\left(\mathrm{V}_{0}\right)$ 残留在内涵体膜, 而另 外一个子复合体 $\left(\mathrm{V}_{1}\right)$ 游离到细胞质中。这一分离导致 $v$-ATPase（质子泵）活性的丧失, 从而降低内 涵体的酸化。碱化的内涵体腔诱导 CD36从内涵体转移到肌膜, 从而产生胰岛素抵抗, 并最终导致心 肌收缩功能障碍。

为了进一步研究CD36和v-ATPase的联系, 第4 章通过在CD36的胞外环引入四半胱氨酸序列并应用 双砷染料而建立了一个新的苂光方法来观察CD36成像。四半胱氨酸序列被编码在CD36胞外环的三个 不同位置从而构造出不同的突变体。其中一个突变体表现了正常的生物学功能, 并且与我们预想的一 样, 用双砷染料染色后在显微镜下观测到 $\mathrm{CD} 36$ 定位在内涵体。通过经典的免疫荧光染色方法, vATPase的两个子复合体分别被不同的抗体染色。在低脂质或高脂质的条件下, $\mathrm{V}_{0}$ 子复合体都与 CD36 的定位重合; 然而 $\mathrm{V}_{1}$ 子复合体仅仅在低脂质的情况下才与 $\mathrm{CD} 36$ 定位重合。这些数据进一步证实了脂 质过载导致v-ATPase两个子复合体的分离。总之, 这种新的方法提供了另外一种研究CD36失调性动态 转运的方法学。

在酵母和肾细胞中, 两个子复合体可逆性的组装/分离被认为是v-ATPase活性的主要调节机制。在 酵母的研究报告中发现了这种循环性的组装/分离是由可利用的葡萄糖来调节。具体来说是高浓度的 葡萄糖条件有利于v-ATPase两个子复合体的组装, 并因此增加了内涵体的酸化。更进一步的观察发 现, 高浓度葡萄糖可刺激脂质过载的心肌细胞中 $\mathrm{v}$-ATPase的重组（第 5 章）。由于脱氧葡萄糖 (2-deoxy-D-glucose) 不能诱导 v-ATPase的重组, 由此可见葡萄糖并未不能直接促使重组而是可能通 过糖酵解代谢作用。与此同时, 一种有效的AMP活化蛋白激酶（AMPK）活化剂A-769662也对脂质诱 导的v-ATPase失活起到恢复作用, 并能够防止过量CD36转移到肌膜, 从而表明AMPK在v-ATPase特定 亚基介导的磷酸化可能参与调节v-ATPase组装/分离。

总的来说, 本论文对脂质过载所导致的心脏收缩功能障碍和糖尿病心肌疾病提出了新的见解。 $\mathrm{v}$-ATPase的失活可能在其激发的一序列病理状态中发挥着关键性的角色。因此, 重新激活v-ATPase的 活性可能促使CD36定位在内涵体, 提高心肌胰岛素的敏感性以及心肌收缩功能, 并最终对抗脂质诱 发的心肌疾病。 


\section{Valorization}

\section{Social and clinical relevance}

Diabetes has now reached epidemic proportions in the world range. Today, 350 million people across the world are living with diabetes and $90 \%$ of the burden is caused by type 2 diabetes. The American National Diabetes Statistics has reported that in the U.S.A. the total prevalence is 29.1 million children and adults, which represents $9.3 \%$ of the population. It is believed that $7 / 10$ of those people have been diagnosed, whereas the remaining people are still undiagnosed. In the Netherlands, one million people suffer from type 2 diabetes. Every year, the amount of diabetic patients increases by 70,000.

Annually there are 4 million deaths due to diabetes in the world. The major cause of mortality in type 2 diabetes is cardiovascular disease. There is increasing evidence that type 2 diabetes is associated with cardiomyopathy, independent of hypertension and coronary artery disease. Furthermore, cardiac insulin resistance and metabolic alterations, especially lipid accumulation in cardiomyocytes (lipotoxicity) are primary causes leading

to cardiac dysfunction. In a broader perspective, there is growing awareness that lipotoxicity in non-adipose tissues such as myocardium is a common denominator of pathology in three inter-related chronic conditions, i.e., type 2 diabetes, obesity and the metabolic syndrome, which together are becoming a major health problem. As a result, the search for new avenues for therapy of lipotoxicity is of great importance for illnesses directly and indirectly affecting proper cardiac functioning.

Clinical research in diabetic humans is to a large extent confined to non-invasive methods, which limit the study of the molecular basis of type 2 diabetes and its complications (i.e., lipid-induced cardiomyopathy). The successful results obtained in this thesis, also including experiments with human cardiomyocytes, highlight a novel molecular target to prevent lipid accumulation, preserve insulin sensitivity, and eventually restore cardiac function in the lipid-overloaded heart. Considering the high occurrence of type 2 diabetes and diabetic cardiomyopathy, our findings hold significant promise to the clinical application and therefore eventually may alleviate the economic burden of the society.

\section{Novelty of the concept}

Current antidiabetic treatments (i.e., biguanides, glucosidase inhibitors, and thiazolidinediones) in humans are only modestly effective, and most of these treatments show complications. A number of studies have indicated some novel pharmaceutical targets with different underlying pharmacological mechanisms. Unfortunately, for none of those targets as new-mechanism based drug has yet reached the patients, mainly due to 
off-target effects.

Already in the nineties, the research group as a part of which I performed my $\mathrm{PhD}$ research, has identified CD36 as the main fatty acid transporter in the heart. Upon lipid oversupply, increased CD36-mediated fatty acid uptake is the key process resulting in cardiac lipid accumulation, and subsequent insulin resistance and contractile dysfunction. Hence, a pharmacological blockade of CD36 could theoretically prevent the maladaptive lipid-induced alterations during lipid oversupply. However, CD36 is not expected to be a suitable drug target, because it has multiple functions not only related to long-chain fatty acid (LCFA) uptake in multiple tissues. Alternatively, given that increased CD36-mediated fatty acid uptake is due to increased CD36 translocation, the CD36 translocation machinery (consisting of over 50 proteins, in analogy to the GLUT4 translocation machinery) could provide further drug targets to limit fatty acid uptake in the lipidoverloaded heart. Yet, the CD36 translocation machinery has remained almost completely unidentified. Moreover, how lipids stimulate CD36 translocation to the sarcolemma was still entirely unknown. However, in this thesis we discovered that as part of the CD36 translocation machinery, the endosomal proton pump, also known as vacuolar-type $\mathrm{H}^{+}$ATPase (v-ATPase) is a key player in regulation of CD36 translocation. More specifically, $\mathrm{v}$-ATPase becomes inhibited during lipid oversupply, which then directly leads to insulin resistance and contractile dysfunction. The potential underlying mechanism of lipidinduced v-ATPase inhibition is further disclosed, and was observed to involve the disassembly of the two sub-complexes of v-ATPase. Hence, strategies to induce reassembly of v-ATPase would result in v-ATPase re-activation and reversal of the subsequent maladaptive lipid-induced alterations. Indeed, pioneering studies described in chapter 5 have already shown that high glucose concentrations induce the re-assembly of $\mathrm{v}$-ATPase. Yet, it seems rather counterintuitive that high glucose infusion would be a part of a novel strategy to combat insulin resistance in the lipid-overloaded diabetic heart. Perhaps, other therapeutic strategies that would selectively upregulate glucose uptake and/or generate intracellularly the acting glucose intermediate would be better suited to re-activate v-ATPase.

\section{Potential application}

V-ATPase is ubiquitously present in mammalian tissues. Consequently, a lack of selectivity for the heart could be a serious barrier to use v-ATPase as drug target. Importantly, v-ATPase has multiple subunits, i.e., at least 14 . Several of these subunits are expressed in different isoforms in different tissues. Therefore, variations in the sensitivity of these isoforms towards drugs might provide an attractive platform for cardiac-specific targeting of v-ATPase. Notwithstanding, it is important to realize that the onset of cardiac insulin resistance goes hand in hand with skeletal muscle and liver insulin resistance. 
Hence, simultaneous re-activation of v-ATPase in these latter tissues, if the same mechanisms of v-ATPase regulation would apply, presumably would be beneficial as well.

In summary, $\mathrm{v}$-ATPase is a promising and innovative target against type 2 diabetes and diabetic cardiomyopathy, even though a long road still awaits its validation, drug discovery, development and clinical testing, before introducing a new medication into the market. Further studies are now ongoing to overexpress GLUT1, the constitutive glucose transporter, or PKD1, a key signaling kinase with a specific involvement in translocation of the main cardiac glucose transporter GLUT4, to increase cardiac glucose uptake. Overexpression of these proteins in lipid-overexposed cardiomyocytes is expected to lead to v-ATPase re-assembly, and could provide further proof-of-principle for v-ATPase reassembly as anti-diabetic strategy. 


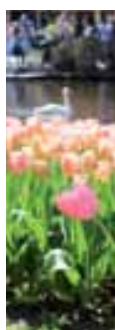

\section{Yilin Liu}

Date of birth: Sep 6 $6^{\text {th }}, 1987$

Nationality: Chinese

Phone: +31687047646

E-mail: liuyilin8796@gmail.com
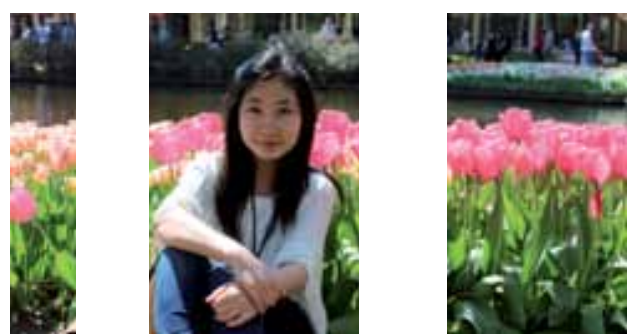

\section{Education}

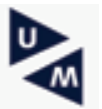

Maastricht University - Maastricht, Netherlands

Oct. 2012 - Sep. 2016

\section{$\mathrm{PhD}$ in Molecular Genetics}

Theme: v-ATPase is key player in lipid-induced cardiomyopathy.

Beijing University of Chinese Medicine - Beijing, China Sep.

2009 - Jun. 2012

M.S. in Pharmacology

Theme: Intervention effects and mechanisms of Xingnaojing Injection

in neuronal apoptosis induced by CORM-2.

Jilin Agricultural University - Jilin, China

Sep. 2005 - Jun. 2009

B.S. in Medicine

Theme: Study on biotransformation of ginsenosides and fermentation activity of ginseng.

\section{Publications}

Y. Liu, D. Neumann, J.F.C. Glatz, J.J.F.P. Luiken. Molecular mechanism of lipid-induced cardiac insulin resistance and contractile dysfunction (In Press, PLEFA)

Y. Liu, L.K.M. Steinbusch, M. Nabben, D. Kapsokalyvas, M. van Zandvoort, P. Schönleitner, G. Antoons, P.J. Simons, D. Chanda, J.F.C. Glatz, D. Neumann, J.J.F.P. Luiken. V-ATPase inhibition underlies lipid-induced cardiomyopathy (Submitted to Diabetes).

Y. Liu, R. Rodriguez-Calvo, X. Zhu, J. Broers, J.F.C. Glatz, J.J.F.P. Luiken, D. Neumann. Fluorescent labeling of fatty acid transporter CD36 in the extracellular domains (In preparation).

D. Chanda, Y. Oligschlaeger, I. Geraets, Y. Liu, X. Zhu, J. Li, M. Miglianico, W.A. Coumans, J.J.F.P. Luiken, J.F.C. Glatz, D. Neumann. 2-Arachidonoylglycerol stimulates glucose uptake and restores insulin sensitivity in cardiomyocytes (Submitted).

Y. Oligschlaeger, M. Miglianico, V. Dahlmans, C.R. Villena, D. Chanda, M.A. Garcia-Gimeno, W.A. Coumans, Y. Liu, J.W. Voncken, J.J.F.P. Luiken, J.F.C. Glatz, P. Sanz, D. Neumann. The interaction between AMPK $\beta 2$ and the PP1-targeting subunit R6 is dynamically regulated by intracellular glycogen content. Biochem J, 2016, 473: 937-947.

Y. Liu, Y. Hong, J. Wang. Intervention mechanisms of Xingnaojing injection with CORM-2 induced apoptosis in neuronal cells through Caspase-dependent mitochondrial pathway. Modernization of TCM, 2013, 15: 1725-1734.

Y. Liu, Y. Hong, J. Wang. Research progress of the novel CO-donor CORMs and its bioactive properties, Progress in Physiological Sciences, 2012, 43: 150-154. 


\section{Presentations}

> SHVM-Society for Heart and Vascular Metabolism, Tromsø, Norway

Oral and poster presentation

Jun. 24-27 2014

$>$ SHVM-Society for Heart and Vascular Metabolism, New York, United States Oral and poster presentation

Oct. 4-7 2015

> CARIM symposium 2015, Maastricht, The Netherlands Poster presentation

Nov. 42015

$>$ American Diabetes Association's 76th Scientific Sessions, New Orleans, United States Oral presentation

Jun. 10-14 2016

\section{Teaching}

Daily supervision of junior master students during their internships

Feb. 2015-Jun. 2015

Daily supervision of senior master students during their internships

Nov. 2015-Jun. 2016

\section{Certificate}

Macroscopic, microscopic and pathologic anatomy of the house mouse

Apr. 2013

Safe Microbiological Techniques

Jun. 2013

Advanced microscopy and vital imaging

Jun 2013

Radiation Protection Level 5B

Feb. 2014

Tutor in the Problem-Based Learning system of Maastricht University

Mar. 2014

Lab animal science Article 9

Jan. 2015

\section{Industry Experience}

Johnson \& Johnson (Department of Pharmacovigilance) - Intern

Jun. 2011 - Sep. 2011

Gohmonaffohmon

Spontaneous AE (adverse effect) reports processing

$>$ Clinical AE Reports processing

$>$ Literature AE Reports processing

Merck Sharp \& Dohme (Department of Regulatory Affairs) - Intern

Mar. 2012 - Aug. 2012

\footnotetext{
(2) MSD $>$ Perform and manage day-to-day product registration, and ensure timely completion

$>$ Ensure operations in compliance with local applicable laws, regulations, and guidelines

Closely follow up product review process

$>$ Properly maintain all regulatory documentation
}

\section{Languages skills}

Chinese: Native

English: $\quad$ Fluent

Dutch: $\quad$ Basics (A1 level) 


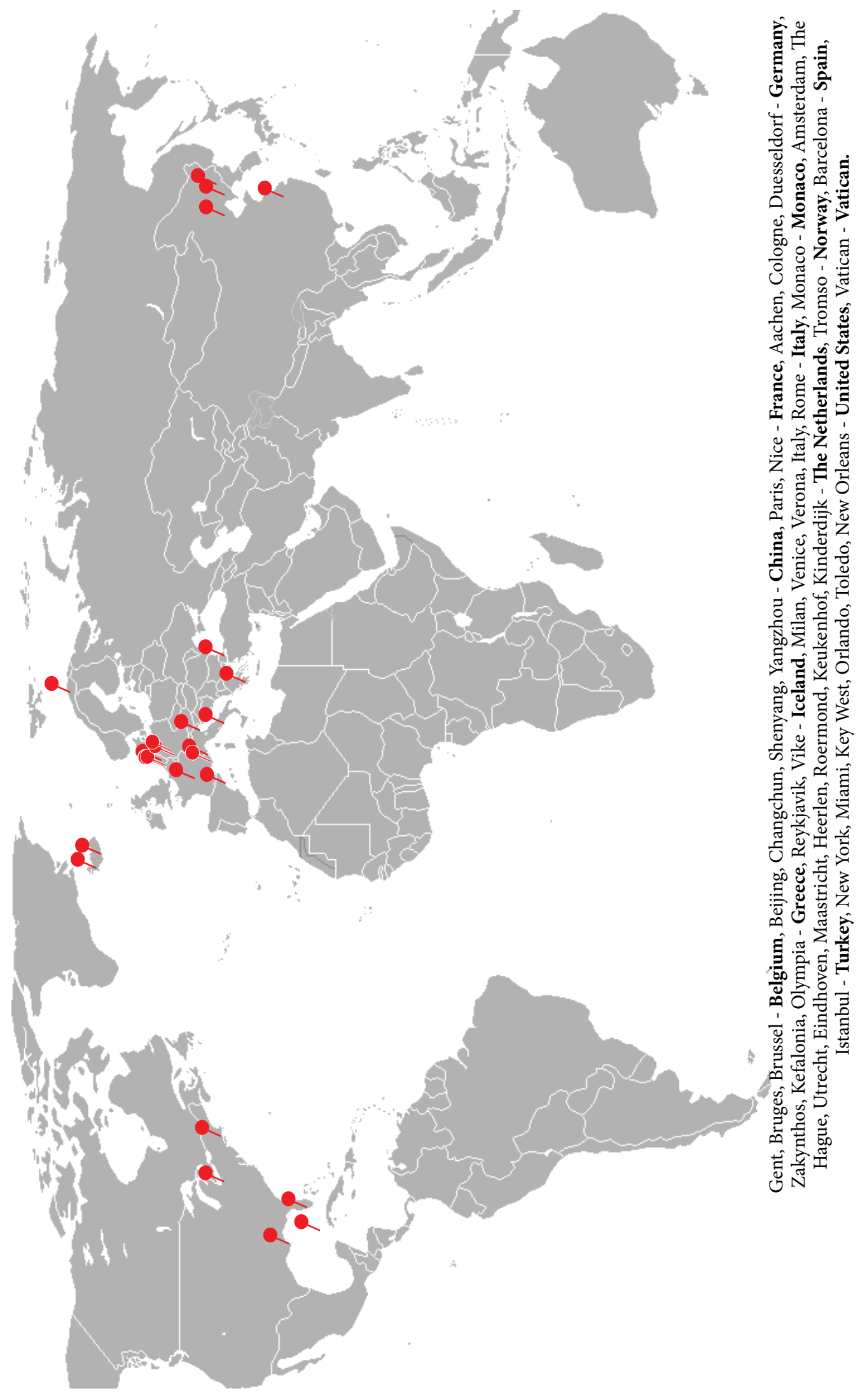



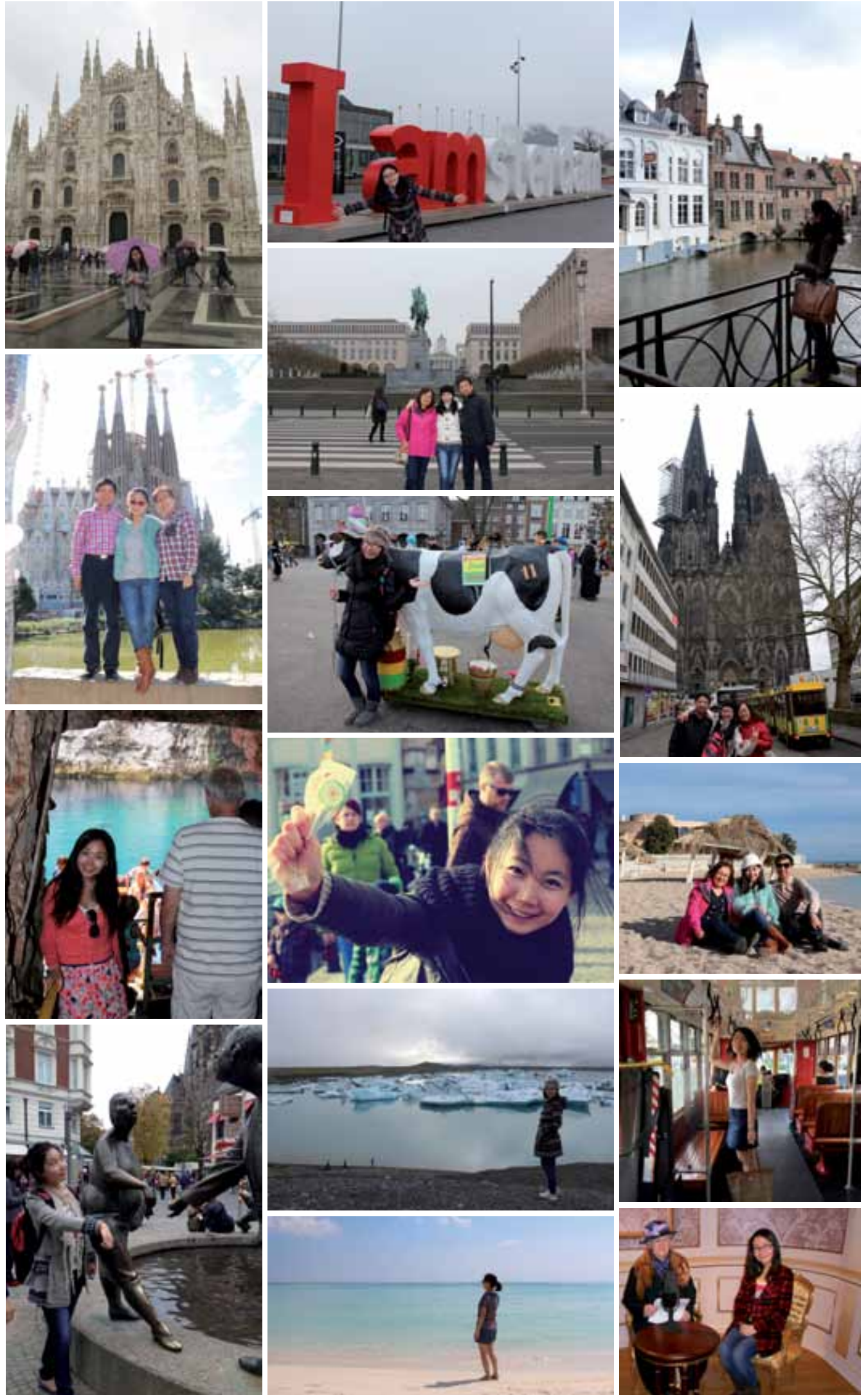


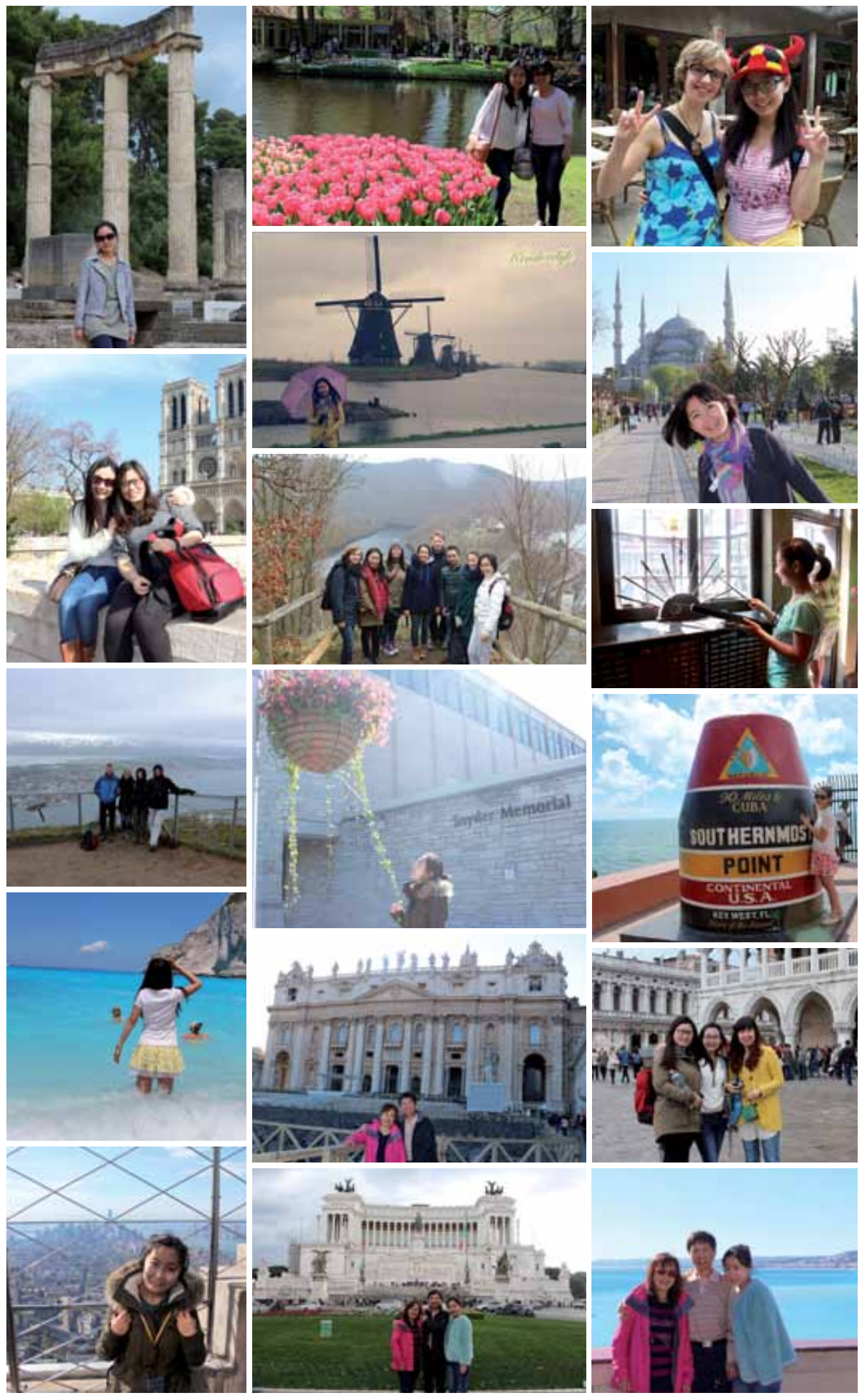




\section{Acknowledgement}

To summarize my work during the last four years, I would like to thank to all the people who have helped and supported me along the journey of my PhD. I am lucky enough to have a good atmosphere to conduct my work and have the support from family, friends, and colleagues. Hereby I would like to express my appreciation and thanks to all of you.

First and foremost, I would like to show my deepest gratitude to my supervisors. Jan, thanks for accepting me as a member in the department of Molecular Genetics. I am greatly indebted for your perceptive advice and shrewd comments throughout my research. During our weekly discussion, I am always inspired by your new ideas, extraordinary patience, and consistent encouragement. Honestly, I cannot finish my PhD project on time without your support.

Joost, you open the door of cardiac metabolism for me. It is my great pleasure to discuss the thoughts, ideas and learn 'CD36' from you. I am really impressed by your enthusiasm and positive attitude about science. Whenever I was frustrated by the experimental results, you can always find a way to cheer me up. I enjoyed chatting with you and your great sense of humor. Thanks for always standing at my side no matter what kind of situation I met throughout the four years. Thanks for being my 'trashcan' and willing to be a patient listener.

Dietbert, I am amazed by your broaden knowledge and deep insight into scientific research. Thanks for always leaving your door open for all my questions and discussions. I have learnt a lot from your side with your diverse background and patient explanations. Thanks for the investment in my research. I really appreciate as one of your group members and the attempt you try to organize our group in an international and active way.

My dear paranymph, Karen. 小庆 (X朱, 朱儿)，我的好 ‘哥们儿’，虽然我们硕士在同一 所大学, 但却从未见过面, 因为你的偶然推荐, 我拿到了马大的offer, 来到马斯特里赫特这座美丽的 中世纪古城开始了我的博士生涯, 同时也开始了我们四年相伴的日子。过去的四年里我们一起走过了 那么多美丽的城市, 一起经历了幸福、痛苦、泪水和欢笑, 当然还有争吵。感谢过去几年的陪伴, 正 是有了你的陪伴与扶持让我度过了人生中最艰难的孤独之路; 感谢过去几年的照顾, 让我这个生活白 痴在远离父母的情况下感受到朋友的温暖; 感谢你对我的 “挑剔”，让任性的我逐渐成长......四年的 时间很快, 转眼间到了毕业季, 在即将分别的时候, 心里有很多不舍, 曾经的争吵竟然都让我觉得很 珍惜, 那些我们一起疯过, 笑过, 走过的日子是那么的清晰, 没有什么华丽的形容词, 只是觉得有 你，真好。我的最佳 “损友” ，愿你实现心中所想，愿你快乐，愿你幸福。

My dear paranymph, Ilvy. I am impressed by your outgoing personality and friendliness. Though it has been just a year since you joined our group, friendship is never based on time. I felt very warm every time when I heard 'If I can help, just let me know'. I enjoyed the time when we were chatting and talking about food. I would never forget your exciting face when we were talking about Peking ducks. Success with your $\mathrm{PhD}$ and I promise I will 
treat you traditional Chinese cuisines once you come to China.

Ricardo, my big brother, I miss the time we spent together in the lab. As your colleague, I really appreciate your efforts for helping me out on my project. As long as you were in the lab, there were always jokes and laugh with happy and lovely atmosphere surrounding us, spicing up our lab work. As your friend, I felt very lucky to have you to be my friend. I enjoyed the moments when we were chatting, gossiping, and sharing ideas. Thanks for the hosting when my family came to visit Barcelona. Let's keep in touch and hopefully I will come back to Barcelona or maybe somewhere in China to meet you again.

My sincerest thanks are extended to our group members. Will, thanks for offering me the help for western blots while I was busy. I really like your positive attitude about life. Whenever you were singing in the lab, I told myself that this is how life should be. Life is a song - sing it. Yeliz, thanks for teaching me isolation and western blot, which were proven to be very curial for my $\mathrm{PhD}$ project. Dipanjan, your broaden knowledge reshaped my impression about scientific research. I have learnt that experiments can be so flexible from you. Marie, thanks for being nice and easy-going. Your diverse background and experience let me know that life can be so colorful and unpredictable. I am still dreaming of learning French and hopefully I can talk to you like an elegant French lady one day. Yvonne, thanks for guiding me for basic lab techniques when I just joined our group. Your serious and conscientious attitude about work also motivates me to move forward continuously. Armand, thanks for your patience and helping me to translate all the Dutch letters. My students, Yvonne and Amber, thanks for trusting my guidance and being so nice as my lab assistants. Yvonne, success in finding a PhD position. Amber, success in your second master and a bright future is waiting for you. Milou, Tari, and Ben, I enjoyed our conversation in the office and wish you all the best in the future.

Moving on to our Molgen group. Willem, I am deeply impressed by your dedication and pursuit of scientific research. Your insistence about science inspires me a lot. Vivian, thanks for helping me out while I was stuck at cloning. Ronit, I have learnt from you that a powerful woman can balance career and family very well. Mike, my neighbor for three years, but still could not name me correctly. Though we did not communicate that much during the last four years, I still feel like you are a very sweet guy. As long as I needed your help, you were always willing and trying efforts to help me. Thanks for your friendliness and earnest. Sofie, thanks for sharing all the information and tips whenever I need. I really appreciate your honesty and kindness. Tom, scientific nerd, do you know you are too mature around your age? I am jealous of your confidence no matter about yourself or your attitude to scientific research. I've already foreseen Prof. Houben and his bright future. Albert, you are such a nice guy with full of passion and positive power. I felt really comfortable to chat with you. Tim, Miranda, Patrick, Marion, Petra, Henny, Hanneke, 
Nadine, Guus, Peggy, Emiel, and Marjo, I enjoyed the moments while we were celebrating all kinds of events in our department and would never forget those nice memories.

From elsewhere in the university, Mieke Henfling, Jos Broers, Miriam Kamps, Dimitris Kapsokalyvas, Marc van Zandvoort, Patrick Schönleitner, Gudrun Antoons, Monika Rech, thank you for your advice, guidance, and help.

Dear Prof. Casper Schalkwijk, Prof. Shengcai Lin, Prof. Ellen Blaak, Dr. Ramon Langen, Dr. Lisa Heather, Dr. Jeanine Prompers, thank you for joining the assessment committee, commenting on my thesis and granting it approval.

感谢过去四年在马城陪伴我的小伙伴们。留学的生活在光鲜亮丽的背后是无尽的孤独和对家人不 断的思念, 正是有了大家的陪伴才让枯燥的生活变得五彩缤纷。正哥和品睿, 你们一直是我眼中的神 仙亚侣, 或许没有那么甜蜜, 或许也少了点浪漫, 但是两个人夫唱妇随, 琴瑟合鸣的样子真的很让人 羡慕。从第一次搬家你们带着我买家具到后来一次次不辞辛劳的过来帮我处理搬家后的琐事，再到之 后处理各种大小麻烦事总是少不了你们的身影。你们如挚友, 如兄长和长姐, 如亲人般陪伴在我的身 边。想念每次饭后富贵小区四人行的日子, 我们畅聊生活, 畅聊未来, 畅谈人生。感恩你们的照顾, 帮助和扶持, 愿你们找到属于自己的一片天空, 执子之手,与子偕老。

敬, 我亲爱的小伙伴, 我们一起走过在马城的四年博士时光, 我们的友情也在相处的过程中逐渐 升温。从最开始我们在维罗纳的彻夜畅聊, 到后来每次遇到事情你的随叫随到, 你的出现总是让人很 暖心。你不是一个善于表达的人, 但你的真诚, 你的行动在不断的证明你对朋友无微不至的关怀。感 恩有你这个朋友, 于欢乐时, 于失意时, 于危难时, 于感动时。在马城的这四年见证了你和伟子的爱 情, 虽然远隔千里, 但爱情的美好并不会随着距离的延伸而褪色, 很羡慕你们, 也很为你开心。愿你 们早日团聚, 永远这样的幸福下去。

婕一和俊芳, 感谢你们多次的款待, 每次搬家你们都来帮忙, 真的很感激。很开心和你们相识, 大家一起玩要, 度过了很多欢乐的时光。看到你们如此的恩爱甜蜜也为你们高兴, 祝福你们在今后的 生活中家庭幸福, 一帆风顺。张硕, 你一直是个很有想法的人, 想法多到有点天马行空, 不过那又如 何, 没有最初的想法又何来之后的成功呢, 很看好你, 继续加油哦。最后感谢你和小安的多次招待。 华仔和徐青, 又一对马城的甜蜜夫妻, 我们一起打牌, 一起聚餐, 一起畅聊, 许多难忘的瞬间, 祝福 你们在未来的日子一切顺利, 生活美满。

姝凝姐姐, 还记得四年前我到达荷兰, 你和姐夫在机场接我的那一幕是我毕生难忘的时刻。当其 他的留学生还站在机场迷茫的时候, 我已经舒服的坐上了开往马城的车。你的嘘寒问暖, 你的关怀备 至, 你的细微照顾都让第一次身处异国他乡的我很感动, 我想亲姐姐也不过如此, 更何况我们是第一 次见面。虽然由于生活在不同的城市, 之后的交流并不多, 但我的感激之情一直都在心里不曾消失。 也许毕业后的我会在其他的国家或城市生活, 但无论我在哪里都会带着我深深的祝福, 祝福姐姐姐夫 无论生活还是工作一切顺利。

亲爱的爸爸妈妈, 感谢你们把我带到这个世界, 感谢你们的养育之恩, 感谢你们的无限支持, 正 是有了你们的教导, 包容, 关怀及呵护才能让我在无忧无虑的环境下成长, 在毫无后顾之忧的情况下 完成学业。由于长期在外的求学生活而不能陪伴在你们的身边, 反而让你们为我牵挂担忧, 心里一直 很愧疚。但是, 爸爸妈妈请相信我, 我已经不再是当年的那颗需要父母照顾的小幼苗了, 也许距离长 成参天大树还有一段路程, 但我会努力, 努力让自己强壮, 努力创造出属于自己的一片天地, 为你们 遮风挡雨。

Once upon a time, I was questioning myself whether it was worth doing this $\mathrm{PhD}$. Finally, I have the answer and it is yes. Yes, I made it. I have been through the most lonely, stressful, and fruitful period in my life. No pain, no gain. I believe these memorable 
experience would inspire me to face all the challenges for the rest of my life and overcome obstacles on the road to success. God bless me......

July, $1^{\text {st }}, 2016$

Maastricht 
